\title{
A Curve-Based Mixed System Rating Method for Unitary Air Conditioners
}

W. Vance Payne

Piotr A. Domanski

U.S. DEPARTMENT OF COMMERCE National Institute of Standards and Technology Building Environment Division Building and Fire Research Laboratory Gaithersburg, Maryland 20899-8631

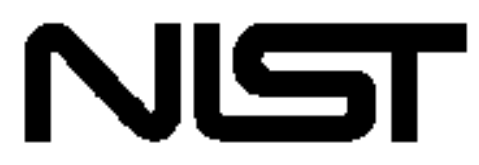

National Institute of Standards and Technology

Technology Administration United States Department of Commerce 


\title{
A Curve-Based Mixed System Rating Method for Unitary Air Conditioners
}

\author{
W. Vance Payne
} Piotr A. Domanski

\begin{abstract}
U.S. DEPARTMENT OF COMMERCE National Institute of Standards and Technology Building Environment Division Building and Fire Research Laboratory Gaithersburg, Maryland 20899-8631
\end{abstract}

May 2005

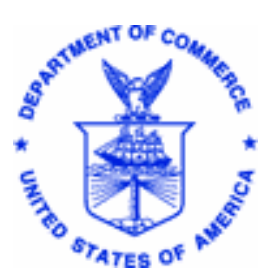

\section{U.S. DEPARTMENT OF COMMERCE}

Carlos M. Gutierrez, Secretary TECHNOLOGY ADMINISTRATION

Phillip J. Bond, Under Secretary of Commerce for Technology National Institute of Standards and Technology Hratch Semerjian, Acting Director 


\section{TABLE OF CONTENTS}

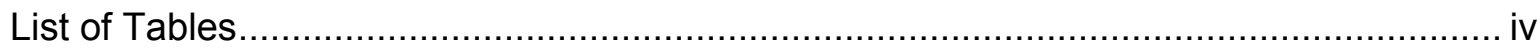

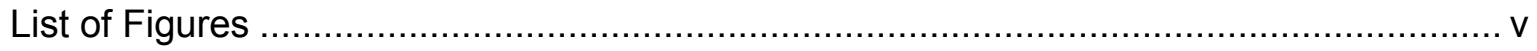

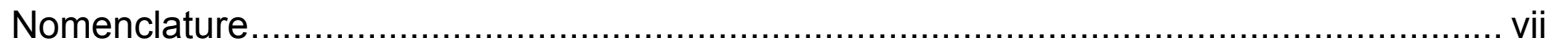

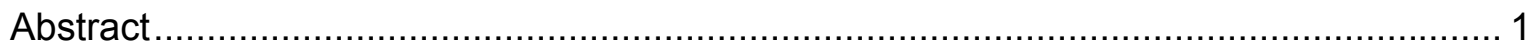

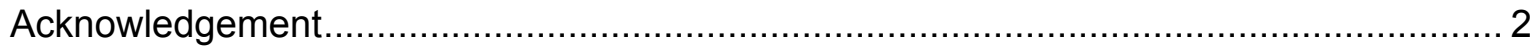

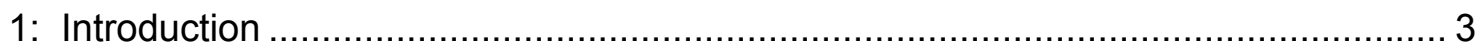

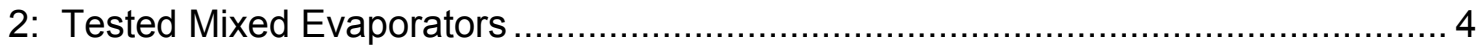

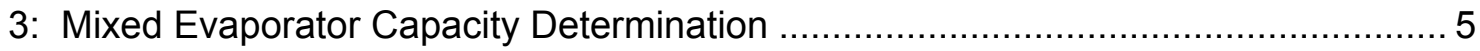

3.1: Experimental setup..................................................................... 5

3.2: Data acquisition and measurement uncertainty ................................... 6

3.3: Test conditions and procedure ...................................................... 7

3.4: Evaporator capacity curve fits and characterization .............................. 8

4: Mixed System Performance Prediction .............................................................. 16

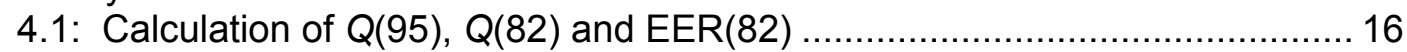

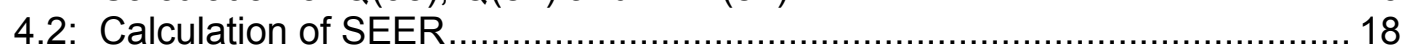

5: NIST Predictions and ARI Database Comparison ............................................ 20

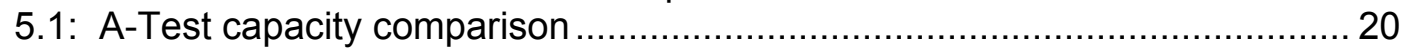

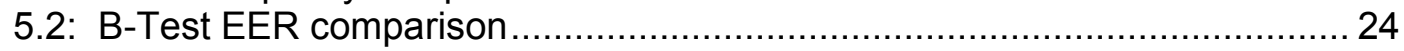

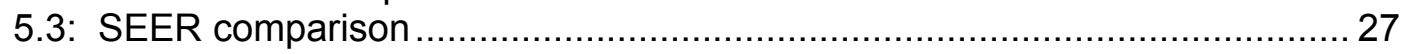

6: Generating an Evaporator Capacity Line Using EVAP-COND ............................. 28

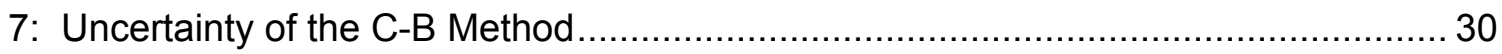

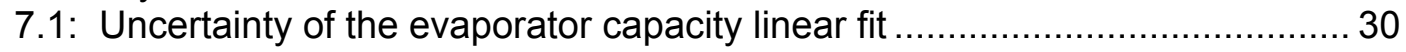

7.2: Uncertainty of C-B Method Q(95) and EER .......................................... 33

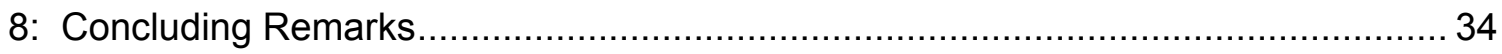

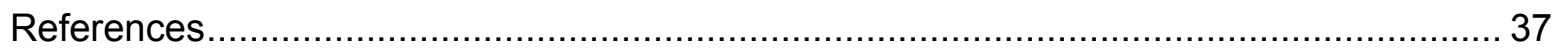

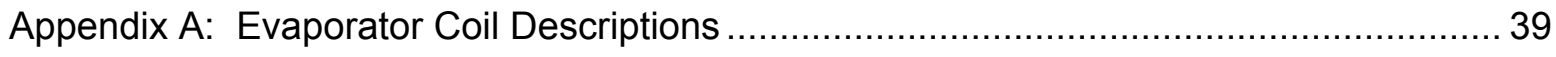

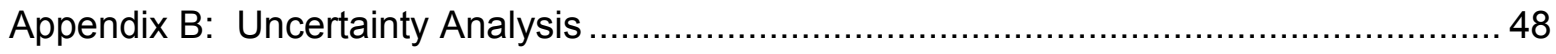

Appendix C: Cooling Measurement Summary Sheets ............................................ 57 


\section{List of Tables}

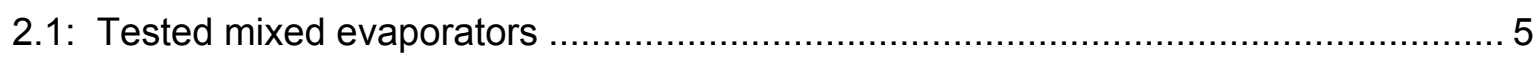

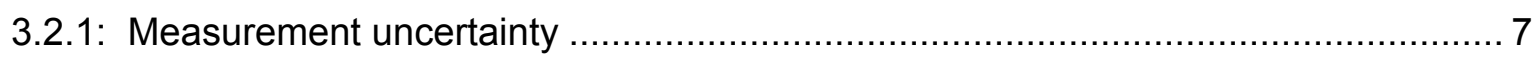

3.3.1: Refrigerant conditions during evaporator tests ...................................... 7

3.4.1: Mixed evaporator capacity linear fit coefficients

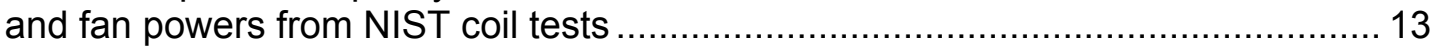

4.1.1: CD Unit linear coefficients for power and capacity

at A-Test and B-Test conditions from ARI .................................................... 16

4.1.2: Mixed system A-Test capacity from the C-B Method ...................................... 17

4.1.3: Mixed system B-Test capacity, power, and EER from the C-B Method................. 18

4.2.1: System classifications for cyclic degradation coefficient analysis...................... 19

4.2.2: Cyclic degradation coefficient values for different system categories.................. 19

4.2.3: Mixed system SEER calculated using statistically

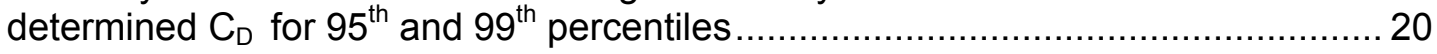

5.1.1: Mixed system A-Test capacities from ARI Tests and C-B Method ....................... 20

5.1.2: Mixed system A-Test capacities from the ARI tests, condensing unit capacities from CD Unit curves, and evaporator capacities from NIST-developed evaporator capacity curves at the ARI-test evaporating temperature.....

5.1.3: Mixed system A-test capacities from ARI values and evaporator capacities from NIST-developed evaporator capacity curves at the ARI-test evaporating temperature....

5.2.1: Mixed system B-Test comparison of ARI tested and C-B Method results .............. 26

5.3.1: SEERs from ARI database and C-B Method ....................................... 27

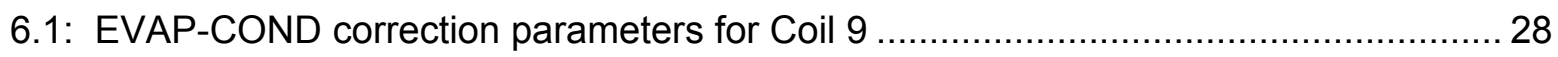

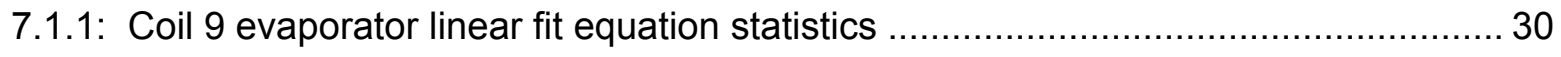

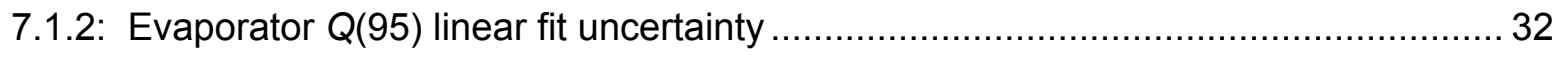

7.2.1: C-B Method $Q(95), Q(82), P(82)$, and EER uncertainty at the $95 \%$ confidence level on the mean value 


\section{List of Figures}

1.1: Graphical illustration of the curve-based rating procedure ................................ 4

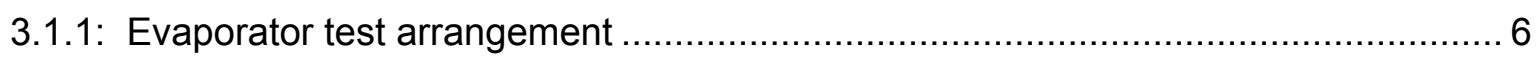

3.4.1: Coil 1 cooling capacity as a function of outlet saturation temperature .................. 8

3.4.2: Coil 2 cooling capacity as a function of outlet saturation temperature .................. 9

3.4.3: Coil 3 cooling capacity as a function of outlet saturation temperature .................. 9

3.4.4: Coil 4 cooling capacity as a function of outlet saturation temperature .................. 10

3.4.5: Coil 5 cooling capacity as a function of outlet saturation temperature .................. 10

3.4.6: Coil 6 cooling capacity as a function of outlet saturation temperature .................. 11

3.4.7: Coil 7 cooling capacity as a function of outlet saturation temperature .................. 11

3.4.8: Coil 8 cooling capacity as a function of outlet saturation temperature ................... 12

3.4.9: Coil 9 cooling capacity as a function of outlet saturation temperature ................... 12

3.4.10: Cooling capacity, $q(95)$, for all evaporators based on NIST linear-fit.................. 14

3.4.11: Airflow rate relative to cooling capacity from the NIST linear

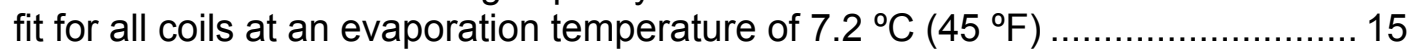

3.4.12: NIST measured SHR as a function of airflow rate per unit of cooling capacity at an evaporator exit saturation temperature of $16.2^{\circ} \mathrm{C}\left(50.0^{\circ} \mathrm{F}\right) \ldots \ldots \ldots \ldots \ldots \ldots \ldots . . . . . . . .15$

5.1.1: Comparison of mixed system A-test capacities from the ARI tests and the C-B method.

5.2.1: Percent differences between ARI Values and the

C-B Method for the EER(82) calculation.

5.3.1: ARI Values and C-B Method SEER/EER Multiplier, EER, and SEER .................. 27

6.1: Coil 9 simulated and measured cooling capacity ........................................ 29

6.2: C-B Method $Q(95)$, EER(82) and SEER ratio with respect to the ARI database using the measured and simulated Coil 9 capacity 
7.1.1: Coil 9 evaporator cooling capacity, confidence and error bands on the mean predicted value of the cooling capacity sampled at a particular evaporator saturation temperature 


\section{Nomenclature}

A

A-Test

B-Test

$\mathrm{C}_{\mathrm{D}}$

C-B Method

CD Unit

CLF

Diff

DOF

EVAP-COND

EER

$\dot{m}$

matched

mixed

n

P

$p(82)$

$P(82)$

$\Delta P$
EVAP-COND air-side heat transfer coefficient correction factor

refers to ARI Standard $210 / 240$ steady-state test conditions of $35^{\circ} \mathrm{C}\left(95^{\circ} \mathrm{F}\right.$ ) outdoor air and $16.7^{\circ} \mathrm{C}\left(80^{\circ} \mathrm{F}\right)$ dry-bulb/ $19.4^{\circ} \mathrm{C}\left(67^{\circ} \mathrm{F}\right)$ wet-bulb indoor air conditions

refers to ARI Standard 210/240 steady-state test conditions of $27.8^{\circ} \mathrm{C}\left(82^{\circ} \mathrm{F}\right)$ outdoor air and $16.7^{\circ} \mathrm{C}\left(80^{\circ} \mathrm{F}\right)$ dry-bulb/ $19.4^{\circ} \mathrm{C}\left(67^{\circ} \mathrm{F}\right)$ wet-bulb indoor air conditions

cyclic degradation coefficient as defined in ARI Standard 210/240-2003

curve-based method as presented in this report

condensing unit, the outdoor section of the split air-conditioner

Cooling Load Factor as defined in ARI Standard 210/240-2003

abbreviation for difference

degrees of freedom

refers to evaporator and condenser simulation software available from NIST

Energy Efficiency Ratio as calculated in ARI Standard 210/240-2003, W/W $(\mathrm{Btu} / \mathrm{W} \cdot \mathrm{h})$

mass flow rate, $\mathrm{kg} / \mathrm{h}(\mathrm{lb} / \mathrm{h})$

refers to a split air-conditioning system, an indoor section/condensing unit combination, which rated performance is determined by laboratory testing; also may refer to the evaporator which is used in the matched system.

refers to a split air-conditioning system, an indoor section/condensing unit combination, which rated performance is not determined by laboratory testing; also may refer to the evaporator which is used in the mixed system.

number of tests or number of data points

electrical power, $\mathrm{W}$

condensing unit power at B-Test condition (indoor fan power not included), W

total power of air conditioner at B-Test condition (condensing unit power plus indoor fan power), $\mathrm{W}$

EVAP-COND refrigerant-side pressure drop correction factor 


\begin{tabular}{|c|c|}
\hline Q & Cooling capacity, W (Btu/h) \\
\hline$q(82)$ & $\begin{array}{l}\text { cooling capacity at B-Test condition without accounting for indoor fan heat } \\
\text { input, W (Btu/h) }\end{array}$ \\
\hline$Q(82)$ & $\begin{array}{l}\text { cooling capacity at B-Test conditions with the indoor fan heat input } \\
\text { accounted for, W (Btu/h) }\end{array}$ \\
\hline$q(95)$ & $\begin{array}{l}\text { cooling capacity at A-Test conditions without accounting for indoor fan heat } \\
\text { input, W (Btu/h) }\end{array}$ \\
\hline$Q(95)$ & $\begin{array}{l}\text { cooling capacity at A-Test conditions with the indoor fan heat input } \\
\text { accounted for, } W(B t u / h)\end{array}$ \\
\hline$\rho$ & correlation coefficient \\
\hline $\mathrm{R}$ & EVAP-COND refrigerant-side heat transfer coefficient correction factor \\
\hline scfm & $\begin{array}{l}\text { standard cubic feet per minute, which is equal to the equivalent volumetric } \\
\text { flowrate of air with a density of } 0.075 \mathrm{lbm} / \mathrm{ft}^{3}\end{array}$ \\
\hline SEER & $\begin{array}{l}\text { Seasonal Energy Efficiency Ratio as defined in ARI Standard 210/240-2003, } \\
\text { Btu/(W·h) }\end{array}$ \\
\hline SHR & sensible heat ratio; the ratio of sensible capacity to total capacity \\
\hline$\hat{\sigma}$ & data standard deviation or fit standard error \\
\hline SSE & sum of squares of the error \\
\hline t or t-value & percentage points of the t-distribution (Ott 1984) \\
\hline ton & cooling or heating capacity equal to $12000 \mathrm{Btu} / \mathrm{h}$ or $3.517 \mathrm{~kW}$ \\
\hline U & quantity's uncertainty \\
\hline
\end{tabular}

\section{Subscripts}

CD condensing unit of the split system air conditioner

cyc cyclic testing

diff difference

dry dry-coil testing

evap refers to the indoor coil or evaporator at saturated refrigerant conditions

fan refers to the indoor coil fan

mixed refers to the evaporator coil alone with respect to a system

ref refrigerant

ss steady-state 


\title{
A Curve-Based Mixed System Rating Method for Unitary Air Conditioners
}

\author{
W. Vance Payne and Piotr A. Domanski \\ National Institute of Standards and Technology
}

\begin{abstract}
The curve-based method was evaluated based on performance predictions and independent laboratory testing for nine mixed systems. Capacity predictions were within $\pm 5 \%$ of the tested values for six of the mixed systems, and four of the SEER predictions were within $\pm 5 \%$ of the tested SEERs. Predictions for SEER showed an under prediction bias due to the wide variation of possible values for the cyclic degradation coefficient $\left(C_{D}\right)$ and the necessity of assuming a conservative value of $C_{D}$ in mixed system rating calculations. This report includes detailed measurement data for the tested evaporators and an uncertainty analysis of the rating methodology.
\end{abstract}

Keywords: air conditioner, cooling capacity, cyclic degradation coefficient, mixed system, rating procedure, SEER 


\section{ACKNOWLEDGEMENT*}

This study was sponsored by the United States Department of Energy, Office of Building Technology, State and Community Programs under contract DE-AI01-99EE27572 under project manager Michael Raymond. Mr. John Wamsley and Mr. Glen Glaeser provided assistance with testing of the evaporator coils, operating the chambers, and analyzing data. Mr. Brian Dougherty provided critical information on values of the degradation coefficient for cyclic operation for different equipment options. Mr. Stefan Leigh, of the NIST Statistical Engineering Division, assisted in the uncertainty analysis. Mr. Tim Corcoran and his staff at Intertek Testing Services (ITS) provided results from system tests. Mr. Michael Woodford and Ms. Sarah Medepalli of ARI provided technical and logistical support. Mr. Chad Kirkwood of United Technologies, Carrier Corporation aided in formulating the scope of this project. The reviewers of the draft of this report were Mr. David Yashar of NIST, Mr. Carl Bergt of Rheem Manufacturing Company, Mr. Chad Kirkwood of United Technologies Carrier Corporation, and Mr. Michael Woodford of the Air-Conditioning and Refrigeration Institute.

\footnotetext{
* Use of Non-SI Units in a NIST Publication: The policy of the National Institute of Standards and Technology is to use the International System of Units (metric units) in all of its publications. However, in North America in the heating, ventilation and air-conditioning industry, certain non-SI units are so widely used instead of SI units that it is more practical and less confusing to include some measurement values in customary units only.
} 


\section{1: INTRODUCTION}

A given condensing unit (outdoor section consisting of a condenser, compressor, and associated tubing) is typically offered on the market in several air-conditioner models, which differ by the indoor sections they employ. For all models, the manufacturers must provide performance information, which consists of the Seasonal Energy Efficiency Ratio (SEER) and capacity at the $35^{\circ} \mathrm{C}\left(95^{\circ} \mathrm{F}\right)$ rating point, $Q(95)$. Federal regulations require that only the highest sales volume indoor-section/outdoor-section combination, referred to as the matched system, be tested in a laboratory to obtain the ratings (CFR 2004a). For other combinations of indoor and outdoor sections, so called mixed systems, the federal regulations allow the use of simplified analytical methodologies upon approval by the U.S. Department of Energy (CFR 2004b).

The most commonly used simplified methodologies for rating mixed systems are those based upon publicly available Q(95) and SEER of the matched systems (e.g., Domanski 1989). The application of these methods requires the rater to predict the capacity of the matched evaporator, which is a major shortcoming because the rater is not often familiar with the matched system product line. Since an inaccurate prediction of the matched evaporator leads directly to inaccurate mixed system ratings, a different rating method that would not include this step, e.g. the performance curve-based method (C-B Method), has the inherent potential to be a better rating approach than the one currently used. Recently, both coil and condensing unit manufacturers expressed interest in using the C-B Method to predict mixed system performance.

Figure 1.1 shows the application of the C-B Method in a graphical form. This method uses linear fits to the cooling capacity for the mixed coil, and cooling capacities, $q(82)$ and $q(95)$, and power, $p(82)$, for the condensing unit (CD Unit). The lines are presented as a function of the compressor suction saturation temperature. Overlapping of the evaporator and CD Unit capacities provides mixed system capacities at $27.8^{\circ} \mathrm{C}\left(82^{\circ} \mathrm{F}\right)$ and $35.0^{\circ} \mathrm{C}\left(95^{\circ} \mathrm{F}\right)$ ambient temperatures. Projecting the saturation temperature corresponding with operation at the $27.8^{\circ} \mathrm{C}\left(82^{\circ} \mathrm{F}\right)$ ambient temperature on the $\mathrm{CD}$ Unit power chart provides the power requirement for the $\mathrm{CD}$ Unit at the $27.8^{\circ} \mathrm{C}\left(82^{\circ} \mathrm{F}\right)$ rating point. Figure 1.1 is convenient for explaining the C-B method. In real applications, this method is best implemented numerically using a computer.

It should be noted that the rating process explained above is exclusive of the indoor fan power. Before the rating of the mixed system is finalized, the indoor fan power must be added to the CD Unit power to produce the power for the system at the $27.8^{\circ} \mathrm{C}\left(82{ }^{\circ} \mathrm{F}\right)$ rating point $P(82)$ The indoor fan heat must also be included as heat reducing the cooling capacities $q(95)$ and $q(82)$ obtained from overlapping the capacity lines of the CD Unit and mixed evaporator to produce actual mixed system capacities, $Q(82)$ and $Q(95)$. The energy efficiency ratio at the $27.8^{\circ} \mathrm{C}$ $\left(82^{\circ} \mathrm{F}\right)$ rating point $(\mathrm{EER}(82))$ can then be calculated using the corrected values of capacity, $Q(82)$, and power, $P(82)$.

$$
\operatorname{EER}(82)=\frac{Q(82)}{P(82)}
$$

To conclude with the SEER calculation, the value of the cyclic degradation coefficient, $C_{D}$, is required. 
The cyclic degradation coefficient, $C_{D}$, has to be obtained from a separate analysis or the default value of 0.25 may be assumed.
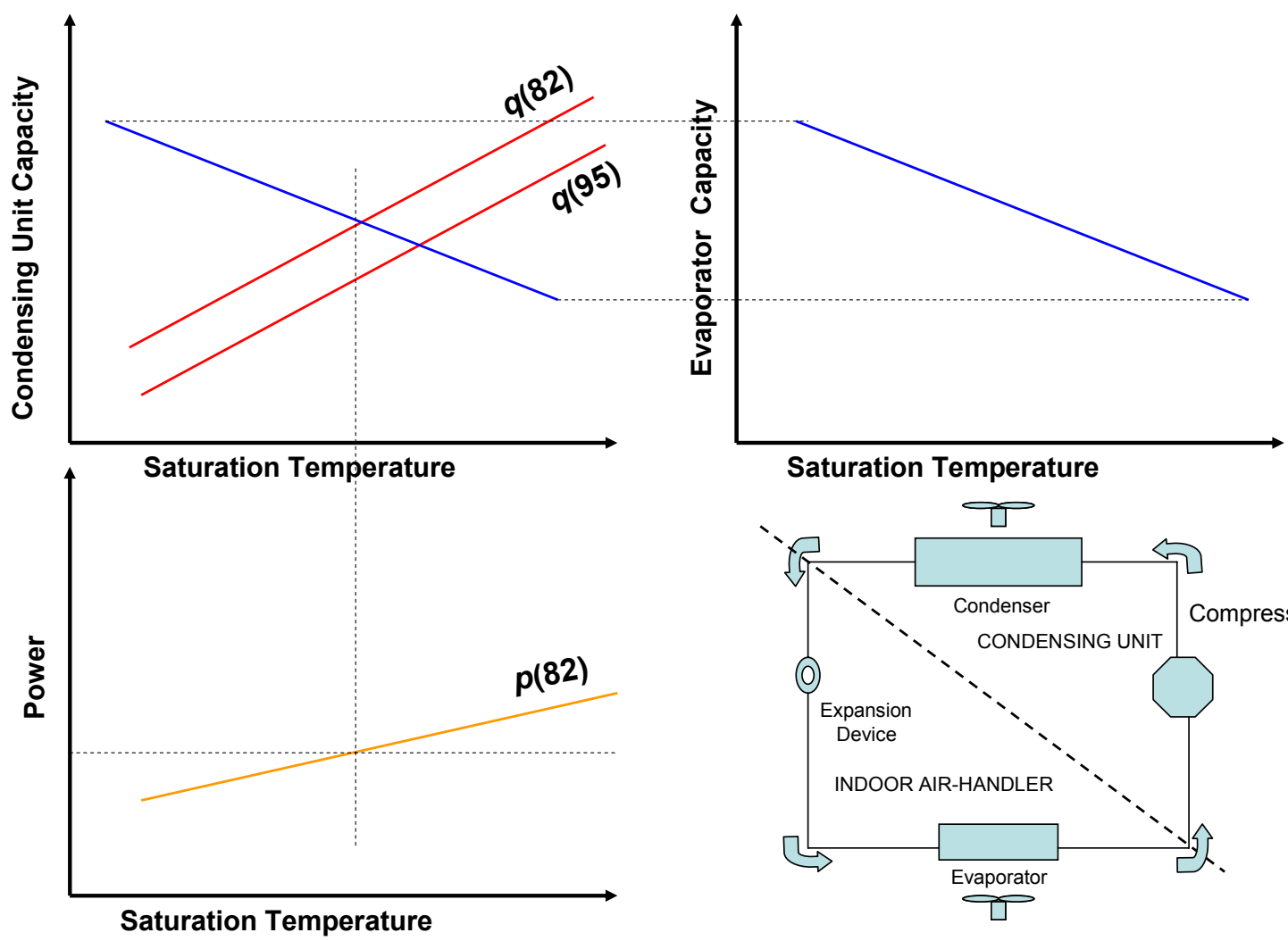
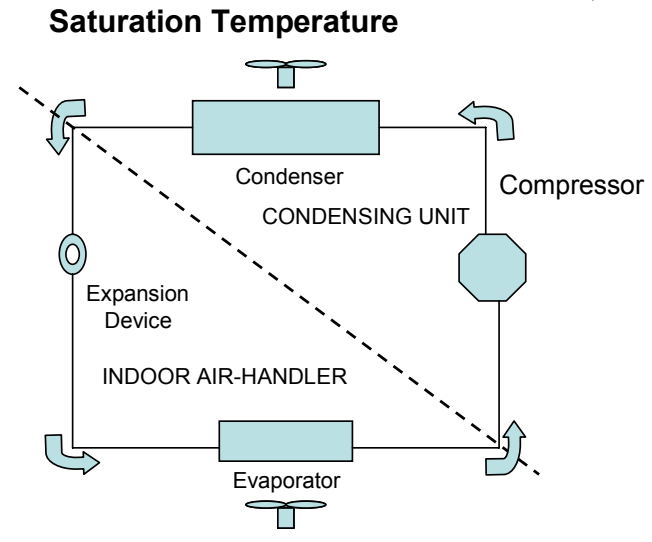

Figure 1.1: Graphical illustration of the curve-based rating procedure

The goal of this study was to evaluate the practicality and accuracy of the curve-based method through its application to nine mixed systems. In this effort, NIST assumed the role of an evaporator manufacturer and developed cooling capacity lines for nine mixed evaporator coils. After obtaining the needed condensing unit performance curves from the Air-Conditioning and Refrigeration Institute (ARI) database, NIST developed mixed system ratings, and then compared them to laboratory derived ratings obtained at an independent testing laboratory for the ARI Unitary Equipment Certification Program.

\section{2: TESTED MIXED EVAPORATORS}

Table 2.1 shows basic information on the tested mixed evaporators. They were manufactured by several different companies and had different capacities. All evaporators were of the finnedtube design. Three evaporators were inclined slabs, four coils were constructed in an A-shape configuration, and one in a semi-A-shape configuration. Three of the coils tested were equipped with a fan and required indoor fan power measurement. The remaining six coils were intended to have field-installed fans. Appendix A presents detailed design data, circuitry configuration, and pictures of the coils. 
Table 2.1: Tested mixed evaporators

\begin{tabular}{|c|c|c|c|c|c|c|}
\hline $\begin{array}{c}\text { Coil } \\
\text { Number }\end{array}$ & $\begin{array}{c}\text { Coil } \\
\text { Designation }\end{array}$ & $\begin{array}{c}\text { Coil } \\
\text { Configuration }\end{array}$ & $\begin{array}{c}\text { Airflow } \\
\text { Direction }\end{array}$ & $\begin{array}{c}\text { Tube Outside } \\
\text { Diameter }\end{array}$ & $\begin{array}{c}\text { Expansion } \\
\text { Device }\end{array}$ & Refrigerant \\
\hline 1 & A01102 & A & Horizontal & $9.5 \mathrm{~mm}(0.375 \mathrm{in})$ & TXV & R22 \\
\hline 2 & $\mathrm{~A} 01070$ & Semi A & Horizontal & $9.5 \mathrm{~mm}(0.375 \mathrm{in})$ & Piston & R22 \\
\hline 3 & $\mathrm{~A} 01148$ & $\mathrm{~A}$ & Upflow & $9.5 \mathrm{~mm}(0.375 \mathrm{in})$ & TXV & R22 \\
\hline 4 & $\mathrm{~A} 01138$ & $\mathrm{~A}$ & Upflow & $9.5 \mathrm{~mm}(0.375 \mathrm{in})$ & Piston & R22 \\
\hline 5 & $\mathrm{~A} 01060^{*}$ & Inclined Slab & $\begin{array}{c}\text { Upflow/ } \\
\text { Horizontal }\end{array}$ & $9.5 \mathrm{~mm}(0.375 \mathrm{in})$ & Piston & R22 \\
\hline 6 & $\mathrm{~A} 01125^{*}$ & Inclined Slab & Horizontal & $9.5 \mathrm{~mm}(0.375 \mathrm{in})$ & TXV & R22 \\
\hline 7 & $\mathrm{H} 5326$ & $\mathrm{~A}$ & Horizontal & $9.5 \mathrm{~mm}(0.375 \mathrm{in})$ & Piston & R22 \\
\hline 8 & $\mathrm{H} 5321$ & $\mathrm{~A}$ & Upflow & $9.5 \mathrm{~mm}(0.375 \mathrm{in})$ & Piston & R22 \\
\hline 9 & $\mathrm{~A} 01154^{*}$ & Inclined Slab & Horizontal & $9.5 \mathrm{~mm}(0.375 \mathrm{in})$ & TXV & R410A \\
\hline
\end{tabular}

*indoor fan included

\section{3: MIXED EVAPORATOR CAPACITY DETERMINATION}

\section{1: Experimental setup}

Figure 3.1.1 shows the experimental setup. The evaporator was installed in the indoor environmental chamber, where air conditions were controlled by a chiller/air handler system. Air was pulled through the evaporator by a centrifugal fan located at the outlet of the nozzle chamber ductwork. The adjacent outdoor chamber housed the water-cooled condensing unit and the laboratory water-chiller. Two different condensing units were used for R22 and R410A evaporators due to lubricant-related considerations. Each condensing unit was equipped with a variable-speed compressor, condenser, and subcooler. The water chiller control system manipulated the temperature and mass flow rate of the water delivered to the condensing unit. The chiller rejected heat to the in-house chilled water loop. Heat rejection was to water and did not require maintaining the outdoor chamber conditions.

The installation of the evaporator and test instrumentation conformed to ASHRAE Standard 371989. We used the air enthalpy method for the primary measurement of the evaporator capacity with the refrigerant enthalpy method providing the secondary measurement. Air dewpoint temperature was measured at the inlet of the evaporator ductwork and in the ductwork after the evaporator and several mixers. Twenty-five node thermocouple grids, located on each side of the evaporator, were used to verify that the air was well mixed at each point. A 25junction thermopile measured the air temperature change across the evaporator. Barometric pressure, evaporator air pressure drop, air temperature and pressure drop in the nozzle, and nozzle temperature were used along with the dew-point measurements to establish the thermodynamic state of the air. The refrigerant enthalpy method required measurement of the evaporator inlet and exit refrigerant temperatures and pressures in addition to mass flowrate. The agreement between the air-side and refrigerant-side methods was always within $4 \%$. 


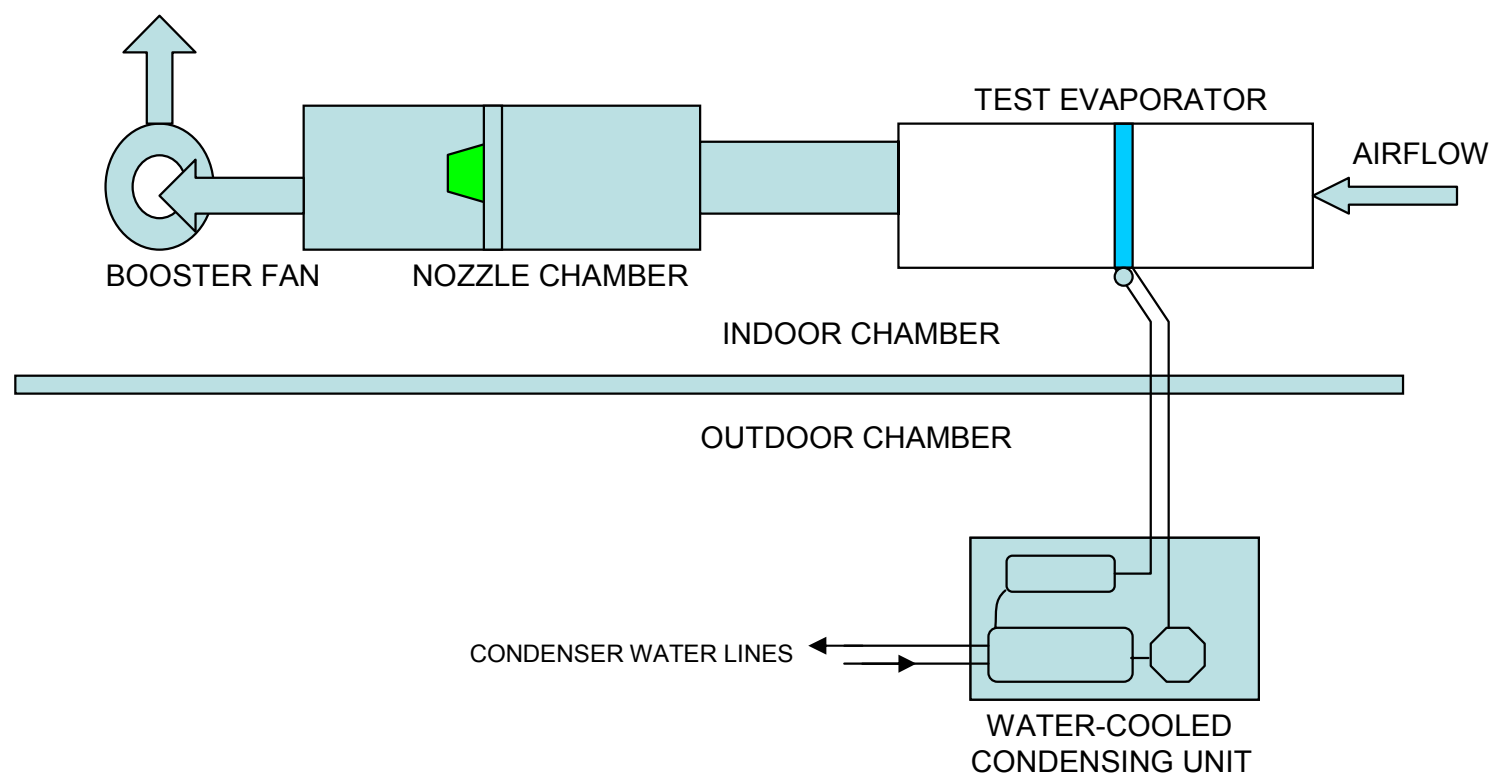

Figure 3.1.1: Evaporator test arrangement

\section{2: Data acquisition and measurement uncertainty}

The measurement points consisted of temperature, pressure, pressure difference, temperature difference, dew-point temperature, fan amps, fan volts, and fan power. Table 3.2.1 lists the measured quantities and their uncertainties for a $95 \%$ confidence limit (two sigma on the mean value) (Taylor and Kuyatt 1994). The uncertainty for the evaporator capacity was calculated using a propagation of uncertainty technique, considering the uncertainty in each of the parameters associated with the capacity measurement. Appendix B explains the application of this technique in more detail. 
Table 3.2.1: Measurement uncertainty

\begin{tabular}{|c|c|c|}
\hline Quantity & Range & Uncertainty \\
\hline Pressure & $\begin{array}{l}\text { 0 kPa to } 3447 \mathrm{kPa} \\
\text { (0 psia to } 500 \mathrm{psia})\end{array}$ & $\begin{array}{l} \pm 3.4 \mathrm{kPa} \\
( \pm 0.5 \mathrm{psi})\end{array}$ \\
\hline Temperature & $\begin{array}{l}-26.1^{\circ} \mathrm{C} \text { to } 93.3^{\circ} \mathrm{C} \\
\left(-15^{\circ} \mathrm{F} \text { to } 200^{\circ} \mathrm{F}\right)\end{array}$ & $\begin{array}{l} \pm 0.3^{\circ} \mathrm{C} \\
\left( \pm 0.5^{\circ} \mathrm{F}\right)\end{array}$ \\
\hline Temperature Difference & $\begin{array}{l}0^{\circ} \mathrm{C} \text { to } 27.8^{\circ} \mathrm{C} \\
\left(0^{\circ} \mathrm{F} \text { to } 50^{\circ} \mathrm{F}\right)\end{array}$ & $\begin{array}{c} \pm 0.3^{\circ} \mathrm{C} \\
\left( \pm 0.5^{\circ} \mathrm{F}\right) \\
\end{array}$ \\
\hline Barometric Pressure & $\begin{array}{c}0 \mathrm{~mm} \mathrm{Hg} \text { to } 1270 \mathrm{~mm} \mathrm{Hg} \\
(0 \mathrm{in} \mathrm{Hg} \text { to } 50 \mathrm{in} \mathrm{Hg})\end{array}$ & $\begin{array}{c} \pm 0.34 \mathrm{~mm} \mathrm{Hg} \\
( \pm 0.0135 \mathrm{in} \mathrm{Hg})\end{array}$ \\
\hline Dew-point Temperature & $\begin{array}{c}0^{\circ} \mathrm{C} \text { to } 50^{\circ} \mathrm{C} \\
\left(32^{\circ} \mathrm{F} \text { to } 122^{\circ} \mathrm{F}\right)\end{array}$ & $\begin{array}{c} \pm 0.2^{\circ} \mathrm{C} \\
\left( \pm 0.4^{\circ} \mathrm{F}\right)\end{array}$ \\
\hline Pressure Difference & $\begin{array}{c}0 \mathrm{~Pa} \text { to } 1244 \mathrm{~Pa} \\
\left(0 \mathrm{in} \mathrm{H}_{2} \mathrm{O} \text { to } 5 \text { in } \mathrm{H}_{2} \mathrm{O}\right)\end{array}$ & $\begin{array}{c} \pm 24.4 \mathrm{~Pa} \\
\left( \pm 0.098 \text { in } \mathrm{H}_{2} \mathrm{O}\right)\end{array}$ \\
\hline Mass Flow & $\begin{array}{c}0 \mathrm{~kg} / \mathrm{h} \text { to } 544.3 \mathrm{~kg} / \mathrm{h} \\
(0 \mathrm{lb} / \mathrm{h} \text { to } 1200.0 \mathrm{lb} / \mathrm{h})\end{array}$ & $\pm 1 \%$ \\
\hline Evaporator Capacity & $\begin{array}{c}5.56 \mathrm{~kW} \text { to } 14.4 \mathrm{~kW} \\
(19 \mathrm{kBtu} / \mathrm{h} \text { to } 49 \mathrm{kBtu} / \mathrm{h})\end{array}$ & $\pm 3 \%$ to $\pm 7 \%$ \\
\hline
\end{tabular}

\section{3: Tests conditions and procedure}

Each evaporator coil was tested at the air volumetric flow rate that was used during mixed system tests carried out at an independent testing laboratory for the ARI certification program. For all tests, constant indoor conditions of $16.7^{\circ} \mathrm{C}\left(80.0^{\circ} \mathrm{F}\right)$ dry-bulb and $15.8^{\circ} \mathrm{C}\left(60.4^{\circ} \mathrm{F}\right)$ dewpoint temperatures were applied according to ARI Standard 210/240 (2003).

On the refrigerant side, the tests were constrained by the refrigerant inlet condition, defined by the liquid line temperature and subcooling, and the outlet condition, defined by the vapor line saturation temperature and superheat. The tests of each evaporator involved three vapor suction line saturation temperatures. The evaporator capacity line was generated as a function of the evaporator exit saturation temperature from these points. Table 3.3.1 lists the refrigerant conditions imposed during the evaporator tests for all coils.

Table 3.3.1: Refrigerant conditions during evaporator tests

\begin{tabular}{|c|c|c|c|}
\hline \multicolumn{2}{|c|}{ Liquid line } & \multicolumn{2}{c|}{ Vapor line } \\
\hline \hline $\begin{array}{c}\text { Temperature } \\
{ }^{\circ} \mathrm{C}\left({ }^{\circ} \mathrm{F}\right)\end{array}$ & $\begin{array}{c}\text { Subcooling } \\
{ }^{\circ} \mathrm{C}\left({ }^{\circ} \mathrm{F}\right)\end{array}$ & $\begin{array}{c}\text { Sat. temperature* } \\
{ }^{\circ} \mathrm{C}\left({ }^{\circ} \mathrm{F}\right)\end{array}$ & $\begin{array}{c}\text { Superheat } \\
{ }^{\circ} \mathrm{C}\left({ }^{\circ} \mathrm{F}\right)\end{array}$ \\
\hline $40.6 \pm 0.8$ & 5.6 to 8.3 & $4.4,7.2,10.0$ & 5.6 to 8.3 \\
$(105.0 \pm 1.5)$ & $(10.0$ to 15.0$)$ & $(40.0,45.0,50.0)$ & $(10.0$ to 15.0$)$ \\
\hline
\end{tabular}

${ }^{*}$ Three nominal conditions

The expansion devices supplied with the various evaporators were removed and replaced by precision needle valves. The liquid line temperature and subcooling, and evaporator superheat were controlled by adjusting the refrigerant charge and by changing the needle valve settings to produce the required superheat at the exit of the evaporator. In addition to compressor speed 
control, an evaporator pressure regulating valve was used to produce the desired exit pressure. Liquid line temperature at the inlet to the expansion valves was also controlled by varying the water flow rates through the liquid cooled subcooler and condensing unit heat exchangers. At least five tests were performed for each evaporator. If the evaporator was equipped with a fan, its power was also measured and recorded for each test.

\section{4: Evaporator capacity curve fits and characterization}

Figures 3.4.1 through 3.4.9 present measured coil capacities, excluding fan heat when a fan was used, as a function of the coil outlet saturation temperature. The figures also include a linear fit to capacity data obtained for each evaporator and the fit coefficients. Examination of the figures indicates that a linear capacity fit is an adequate representation of the measured data. Table 3.4.1 summarizes the cooling capacity linear slopes and intercepts. Appendix C gives detailed data summaries of the tests performed for each coil.

We may note that the presented evaporator test data - including the performance lines - refers to the evaporator exit saturation temperature. The CD Unit curves also use the evaporator exit saturation temperature to calculate cooling capacity; i.e., they include the effect of refrigerant pressure drop and heat transfer in the suction line.

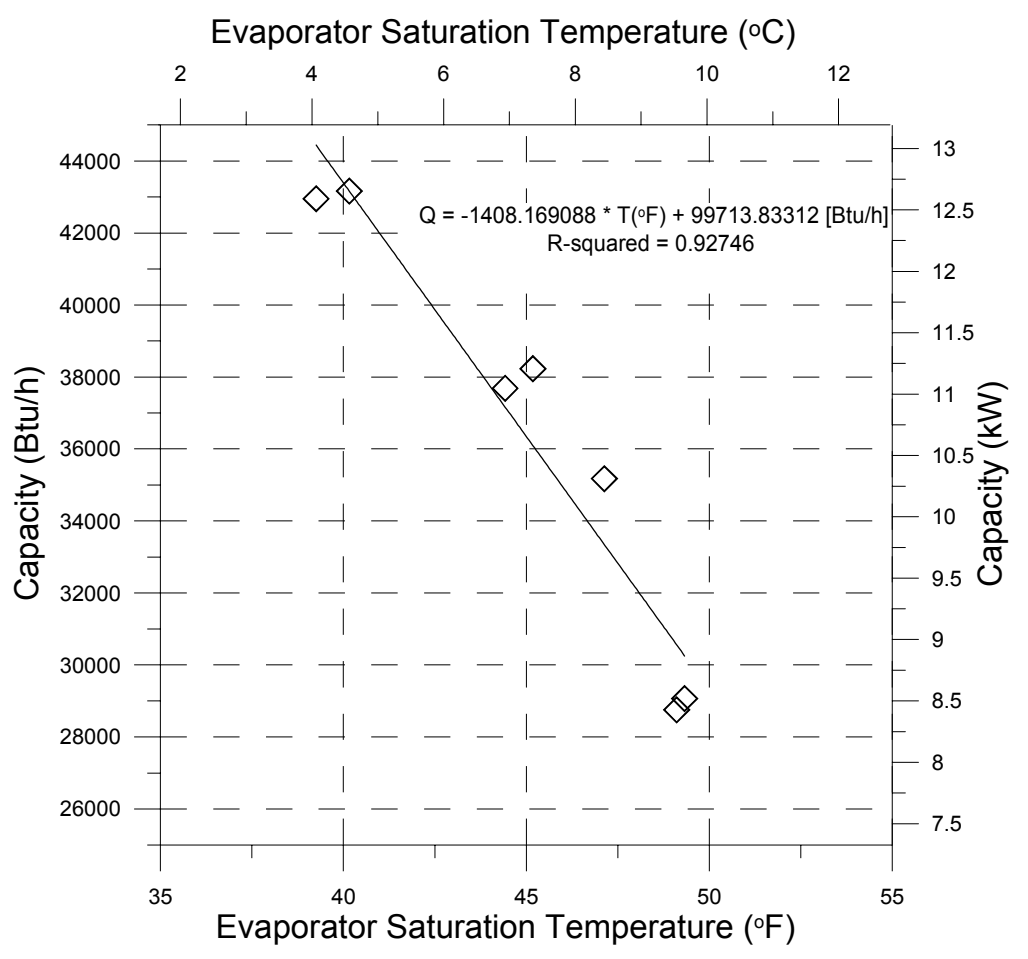

Figure 3.4.1: Coil 1 cooling capacity as a function of outlet saturation temperature 


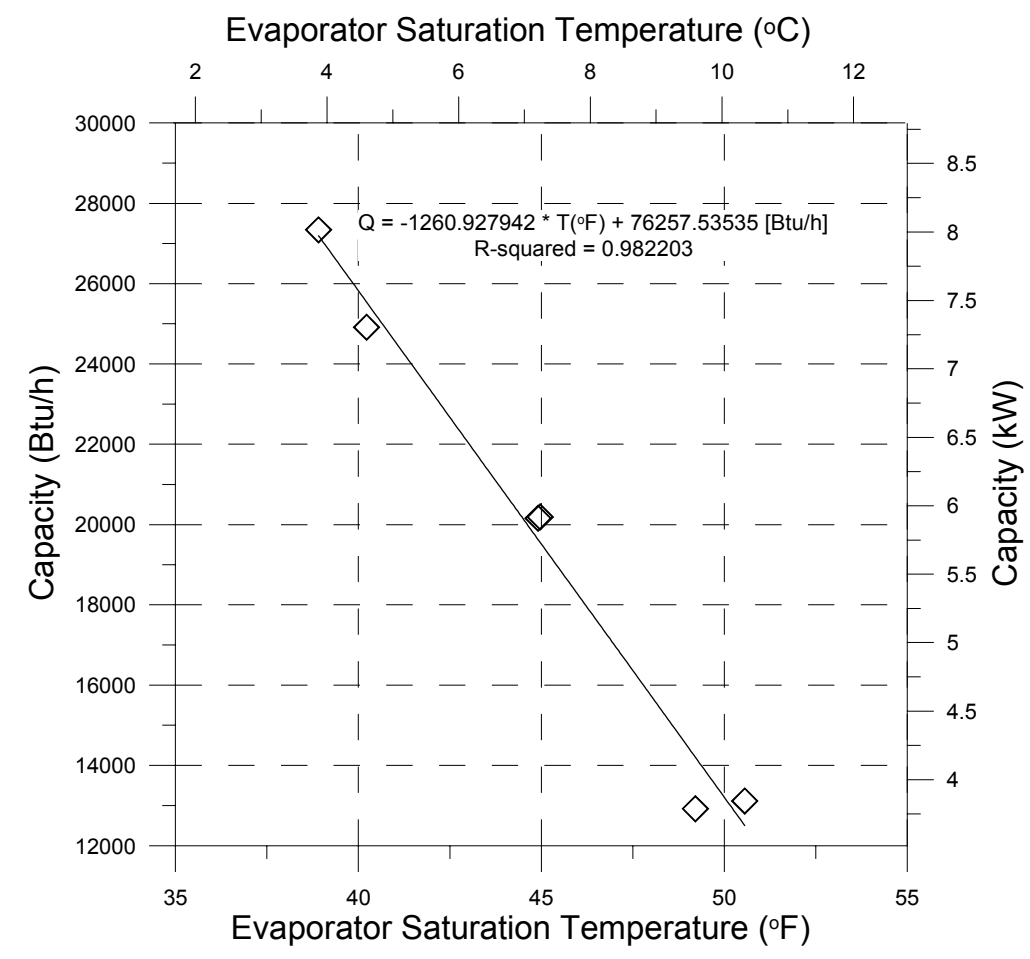

Figure 3.4.2: Coil 2 cooling capacity as a function of outlet saturation temperature

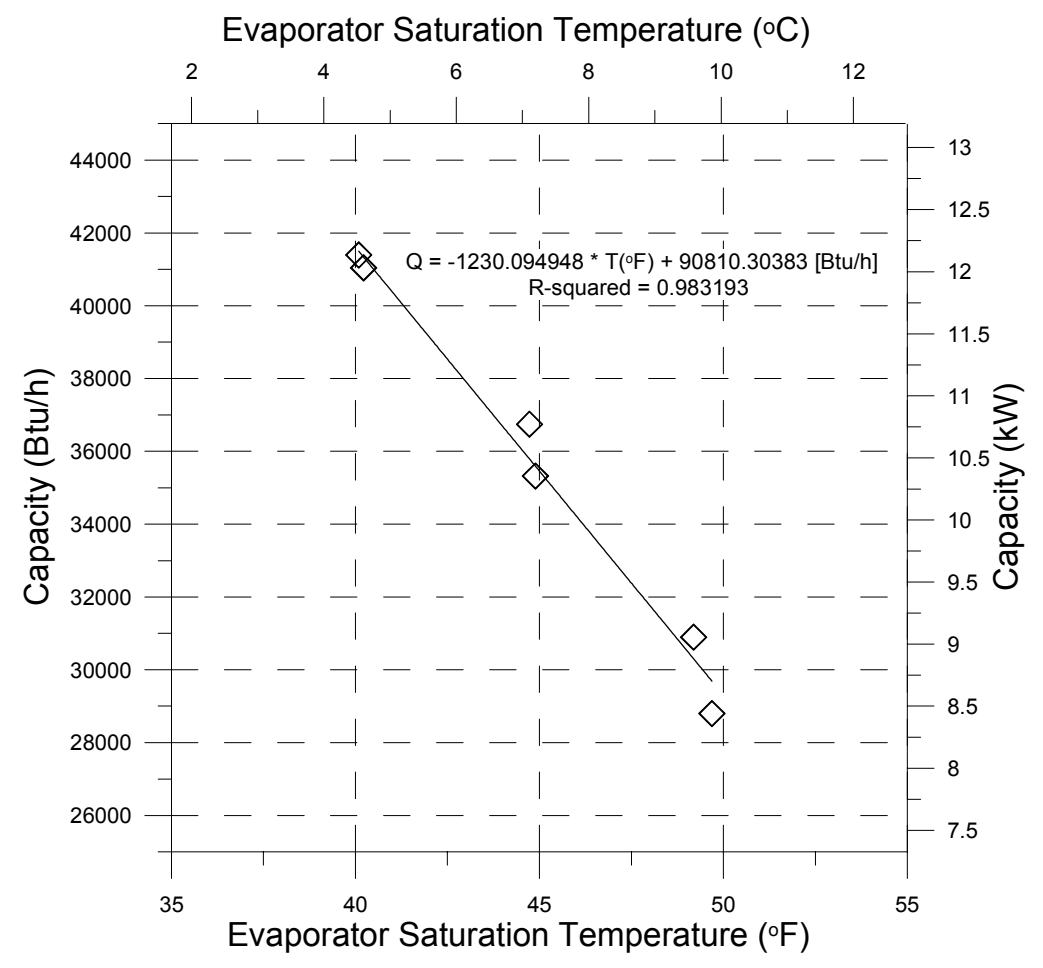

Figure 3.4.3: Coil 3 cooling capacity as a function of outlet saturation temperature 


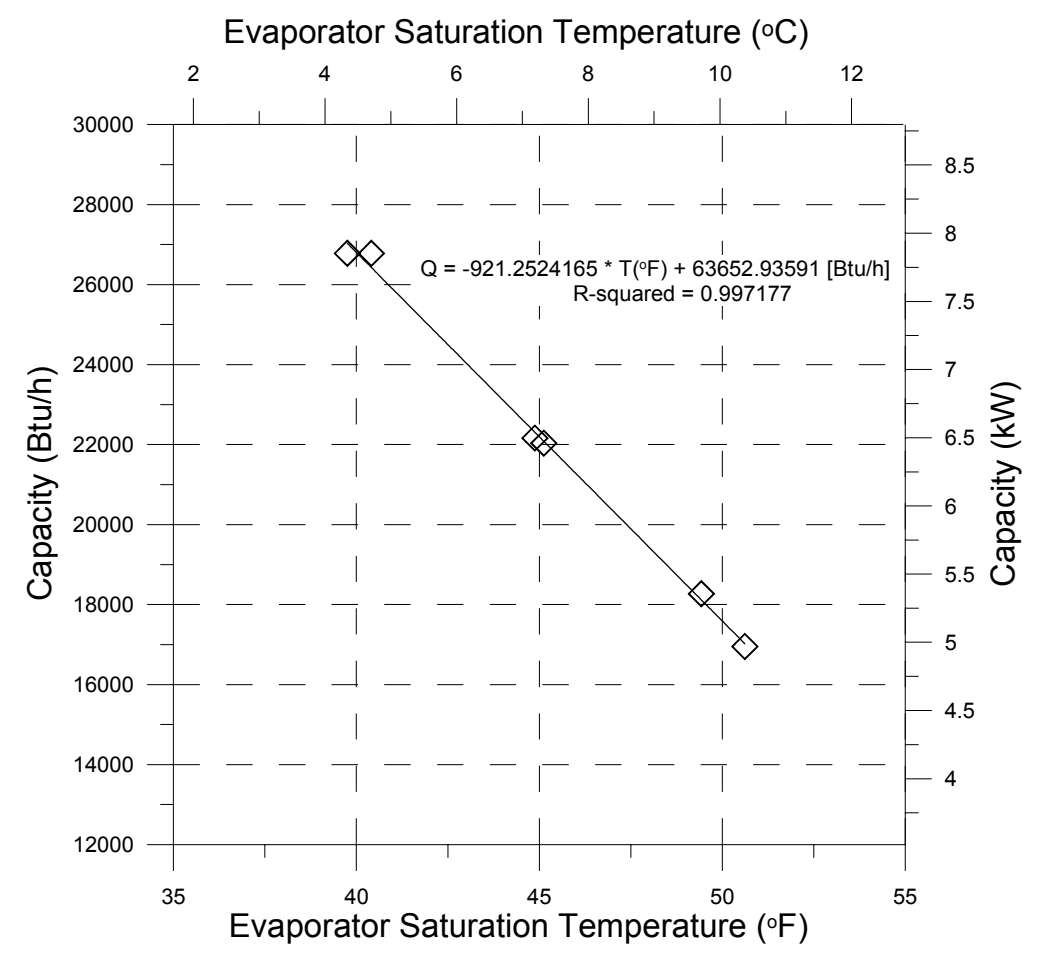

Figure 3.4.4: Coil 4 cooling capacity as a function of outlet saturation temperature

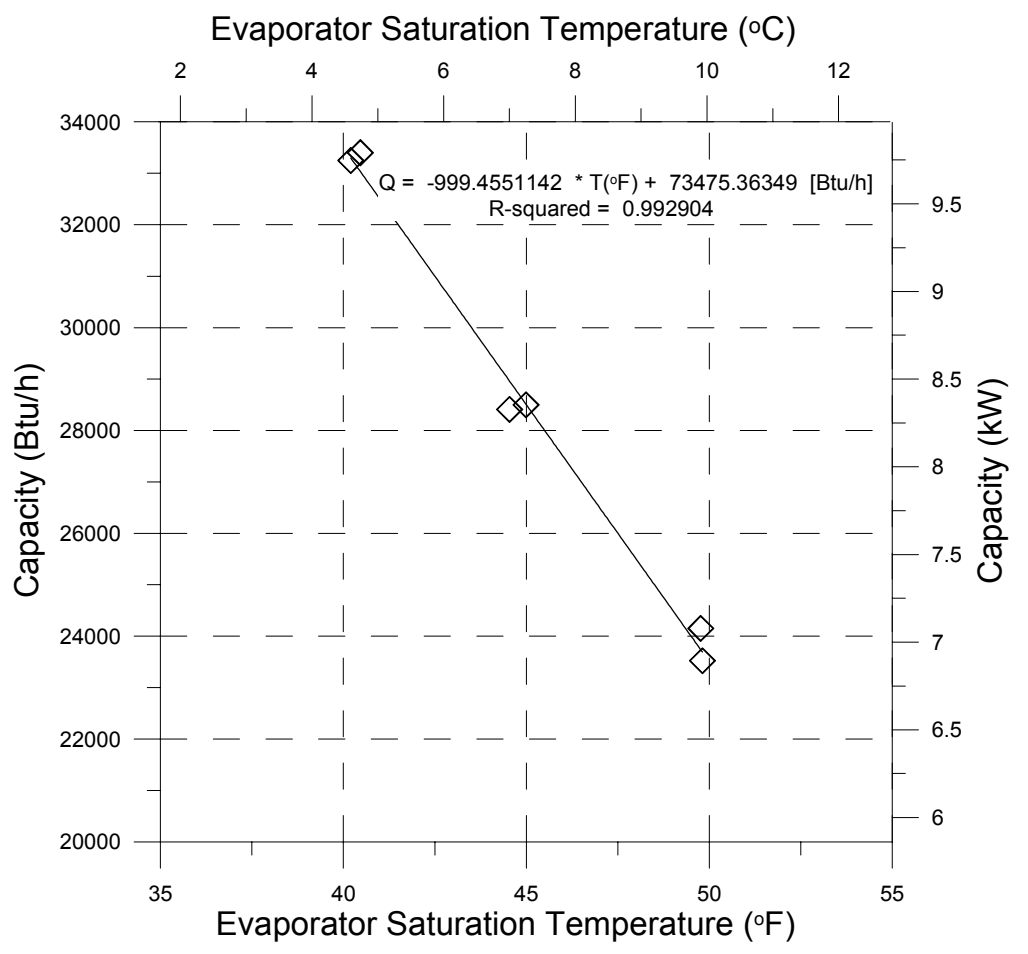

Figure 3.4.5: Coil 5 cooling capacity as a function of outlet saturation temperature 


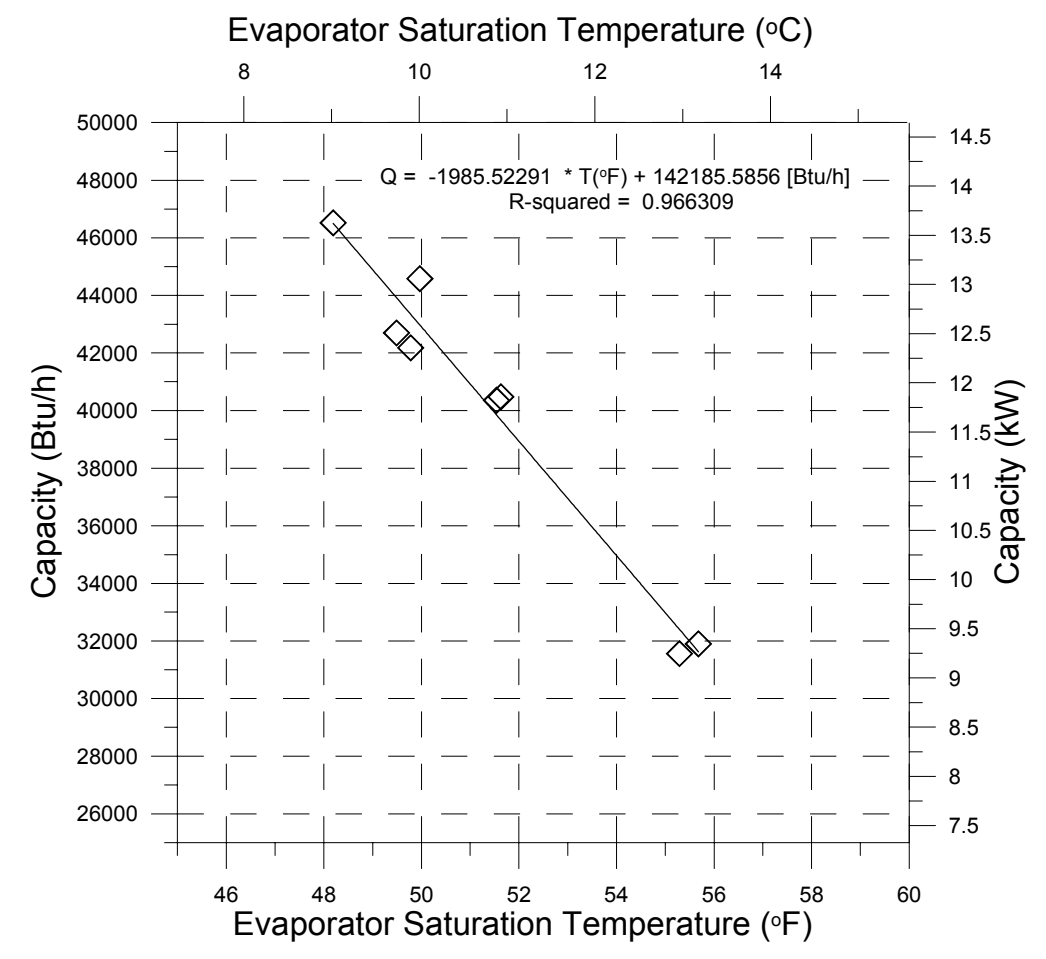

Figure 3.4.6: Coil 6 cooling capacity as a function of outlet saturation temperature

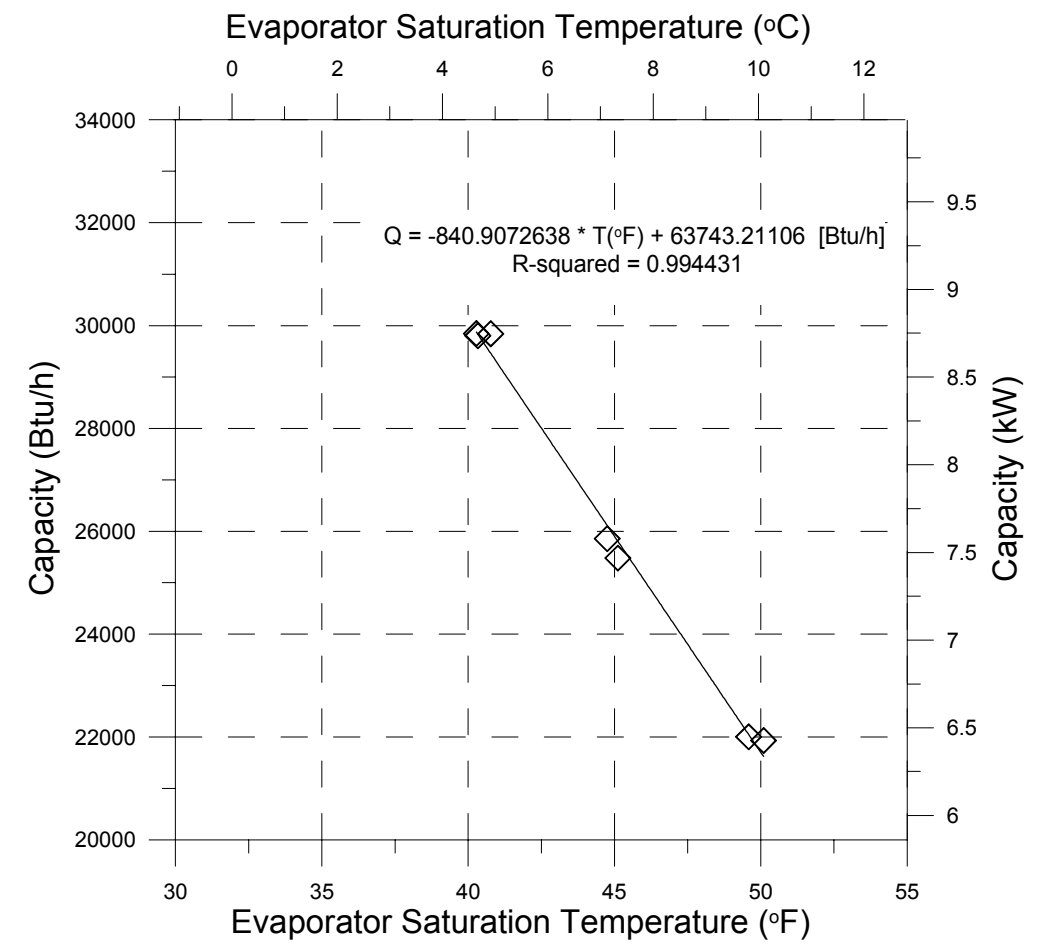

Figure 3.4.7: Coil 7 cooling capacity as a function of outlet saturation temperature 
Evaporator Saturation Temperature $\left({ }^{\circ} \mathrm{C}\right)$

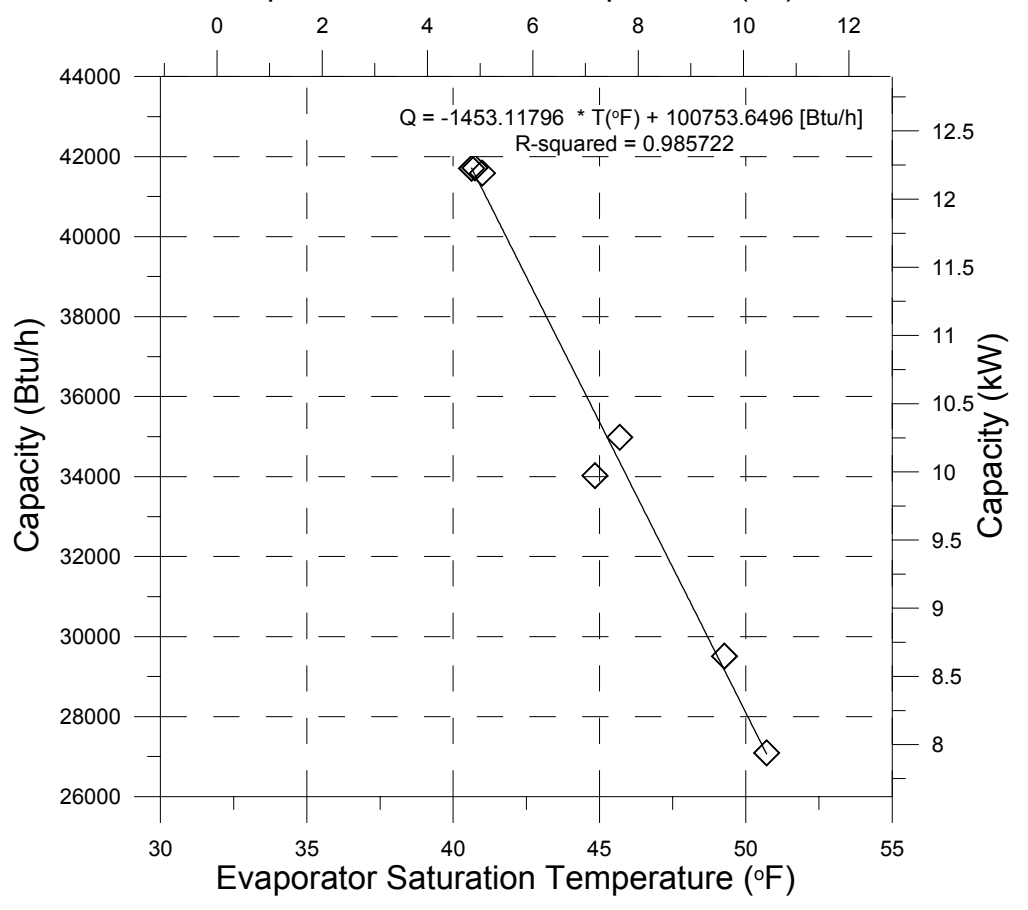

Figure 3.4.8: Coil 8 cooling capacity as a function of outlet saturation temperature

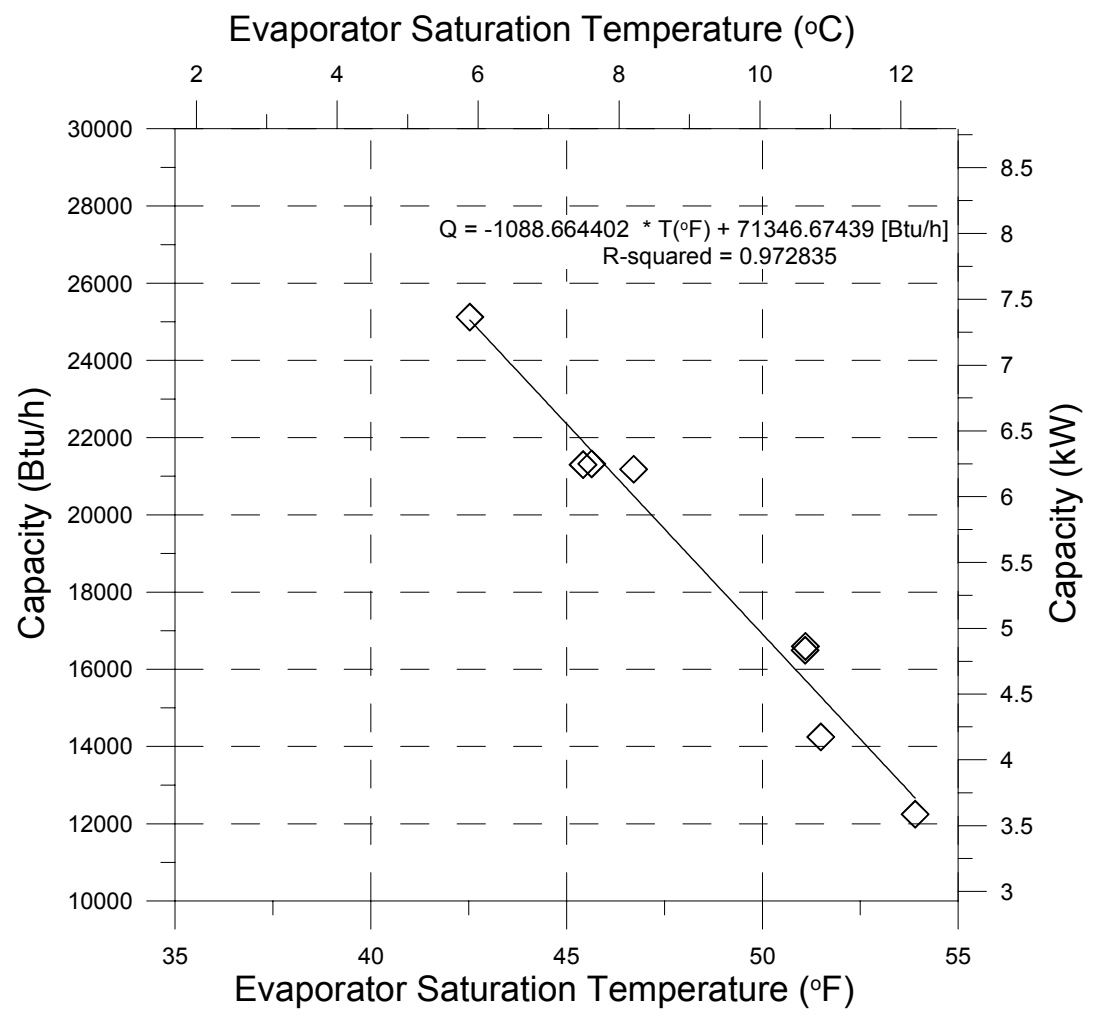

Figure 3.4.9: Coil 9 cooling capacity as a function of outlet saturation temperature 
Table 3.4.1: Mixed evaporator capacity linear fit coefficients and fan powers from NIST coil tests

\begin{tabular}{|c|c|c|c|c|}
\hline \multirow{2}{*}{$\begin{array}{c}\text { Coil } \\
\text { Number }\end{array}$} & \multirow{2}{*}{$\begin{array}{c}\text { Evaporator } \\
\text { Airflow } \\
\mathrm{m}^{3} / \mathrm{h} \text { (scfm) }\end{array}$} & \multirow{2}{*}{$\begin{array}{c}\text { Fan } \\
\text { Power } \\
\text { W }\end{array}$} & \multicolumn{2}{|c|}{ Cooling Capacity Linear Coefficients } \\
\hline & & & $\begin{array}{c}\text { Slope } \\
\mathrm{W} /{ }^{\circ} \mathrm{C}\left(\mathrm{Btu} / \mathrm{h} \cdot{ }^{\circ} \mathrm{F}\right)\end{array}$ & $\begin{array}{c}\text { Intercept } \\
\mathrm{W}(\text { Btu/h) }\end{array}$ \\
\hline 1 & $1699(1000)$ & 0 & $-742.8(-1408.17)$ & $29223.85(99713.83)$ \\
\hline 2 & $1368(805)$ & 0 & $-665.1(-1260.93)$ & $22348.89(76257.54)$ \\
\hline 3 & $1621(954)$ & 0 & $-648.9(-1230.09)$ & $26613.89(90810.3)$ \\
\hline $4^{*}$ & $1342(790)$ & 0 & $-486.0(-921.25)$ & $18654.84(63652.94)$ \\
\hline 5 & $1279(753)$ & 271 & $-527.2(-999.46)$ & $21533.51(73475.36)$ \\
\hline 6 & $1954(1150)$ & 784 & $-1047.4(-1985.52)$ & $41670.51(142185.6)$ \\
\hline 7 & $2047(1205)$ & 0 & $-443.6(-840.91)$ & $18681.3(63743.21)$ \\
\hline 8 & $2360(1389)$ & 0 & $-766.6(-1453.12)$ & $29527.99(100753.65)$ \\
\hline 9 & $849(500)$ & 364 & $-574.3(-1088.66)$ & 20909.65 (71346.67) \\
\hline
\end{tabular}

* Coil 4 airflow was too high and results cannot be compared directly to ARI tests

Figures 3.4.10 thru 3.4.12 help to characterize and contrast the performance of the nine evaporators with respect to one another. Figure 3.4.10 shows the cooling capacity of each coil as a function of the evaporator saturation temperature generated from the capacity linear fits at an evaporator temperature of $7.2^{\circ} \mathrm{C}\left(45^{\circ} \mathrm{F}\right)$. The plot indicates the relative values of a change in capacity with respect to a change in evaporation temperature (slope) for each coil.

Figure 3.4.11 presents the airflow rate per unit of capacity for all coils, which is an indication of air temperature change across the coil. These values are from NIST tests at an evaporator saturation temperature of $10^{\circ} \mathrm{C}\left(50^{\circ} \mathrm{F}\right)$. All NIST tests were intended to be performed at the ARI Test airflow rates. Inadvertently, Coil 4 airflow was $262 \mathrm{~m}^{3} / \mathrm{h}(154 \mathrm{scfm})$ higher than the ARI Tests. Consequently, NIST capacity measurements and predictions for Coil 4 were significantly higher (approximately $24 \%$ ) than would have been the case with correct lower airflow rate. The results for Coil 4 have been included in this report, but they cannot be directly compared with the ARI Tests. The airflow rates at the other tests differed from the ARI Test values on average by $0.14 \%$ with a standard deviation of $1.8 \%$. The average airflow rate per unit cooling capacity was $0.1852 \mathrm{~m}^{3} / \mathrm{W}$ h $(383.5 \mathrm{scfm} / \mathrm{ton})$ with a standard deviation of 0.0518 $\mathrm{m}^{3} / \mathrm{W} \mathrm{h}(107.1 \mathrm{scfm} / \mathrm{ton})$. Coil 6 had the smallest airflow rate per unit capacity $\left[0.1261 \mathrm{~m}^{3} / \mathrm{W} \mathrm{h}^{\mathrm{h}}\right.$ (262 scfm/ton)] as well as the largest change in cooling capacity with respect to a change in evaporator saturation temperature (see Figure 3.4.10). Coil 6 also had the lowest sensible heat ratio (SHR) of 0.710 (Fig. 3.4.12). Fig. 3.4.12 shows a linear trend of SHR with respect to airflow rate per unit capacity, which is consistent with general experience. The high sensible heat ratios were due to the high evaporating temperature of $10^{\circ} \mathrm{C}\left(50^{\circ} \mathrm{F}\right)$. At the lowest evaporating temperatures tested at NIST, the highest SHR's occurred for Coils 2, 7, and 8 with values of $0.77,0.81$, and 0.78 , respectively. All other coils had SHR's less than $75 \%$ at the lowest tested evaporating temperature. 


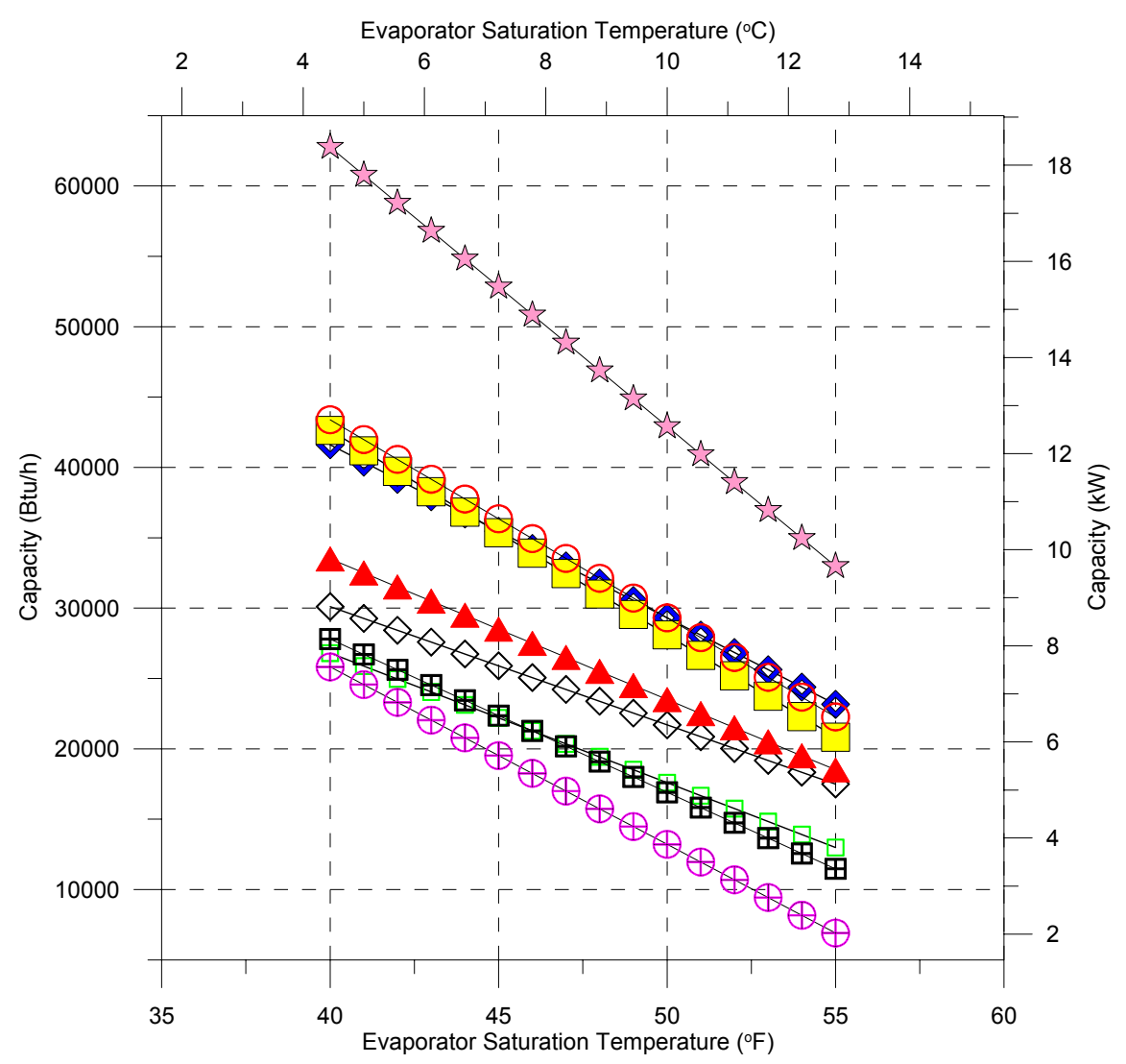

\begin{tabular}{ll}
\hline R22, Coil \#1, Horizontal, A-Coil \\
$\diamond$ R22, Coil \#2, Horizontal, U-Coil Slab \\
$\diamond$ R22, Coil \#3, A-Coil, Upflow \\
$\square$ R22, Coil \#4, A-Coil, Upflow \\
$\square$ R22, Coil \#5, Horizontal, Tilted Slab
\end{tabular}

$\triangle$ R22, Coil \#6, Horizontal, Tilted Slab

औ R22, Coil \#7, Horizontal, A-Coil

田 R22, Coil \#8, A-Coil, Upflow

$\bigoplus$ R410A, Coil \#9, Horizontal, Tilted Slab

Figure 3.4.10: Cooling capacity, $q(95)$, for all evaporators based on the NIST linear fit 


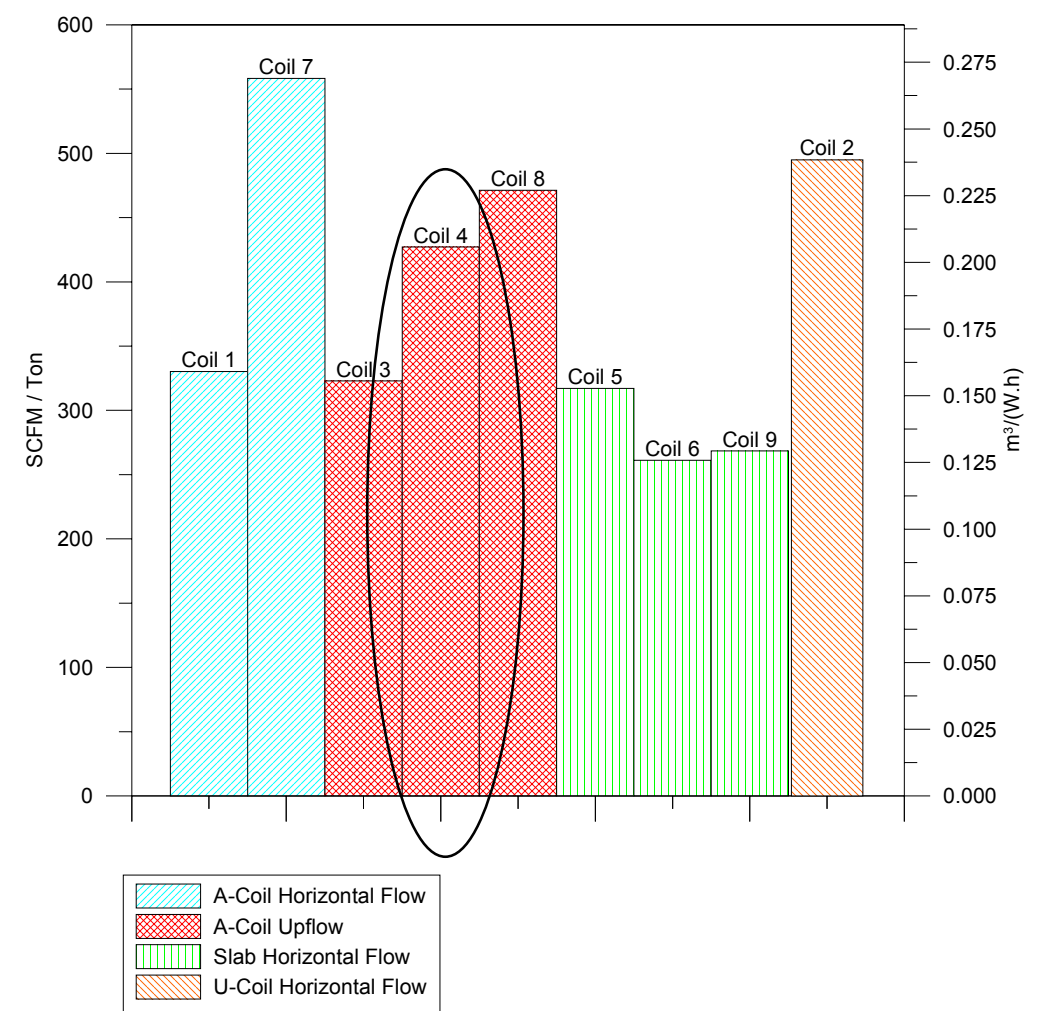

Figure 3.4.11: Airflow rate relative to cooling capacity from the NIST linear fit for all coils at an evaporation temperature of $7.2^{\circ} \mathrm{C}\left(45^{\circ} \mathrm{F}\right)$

(Note: Coil 4 airflow was too high and results cannot be compared directly to ARI tests)

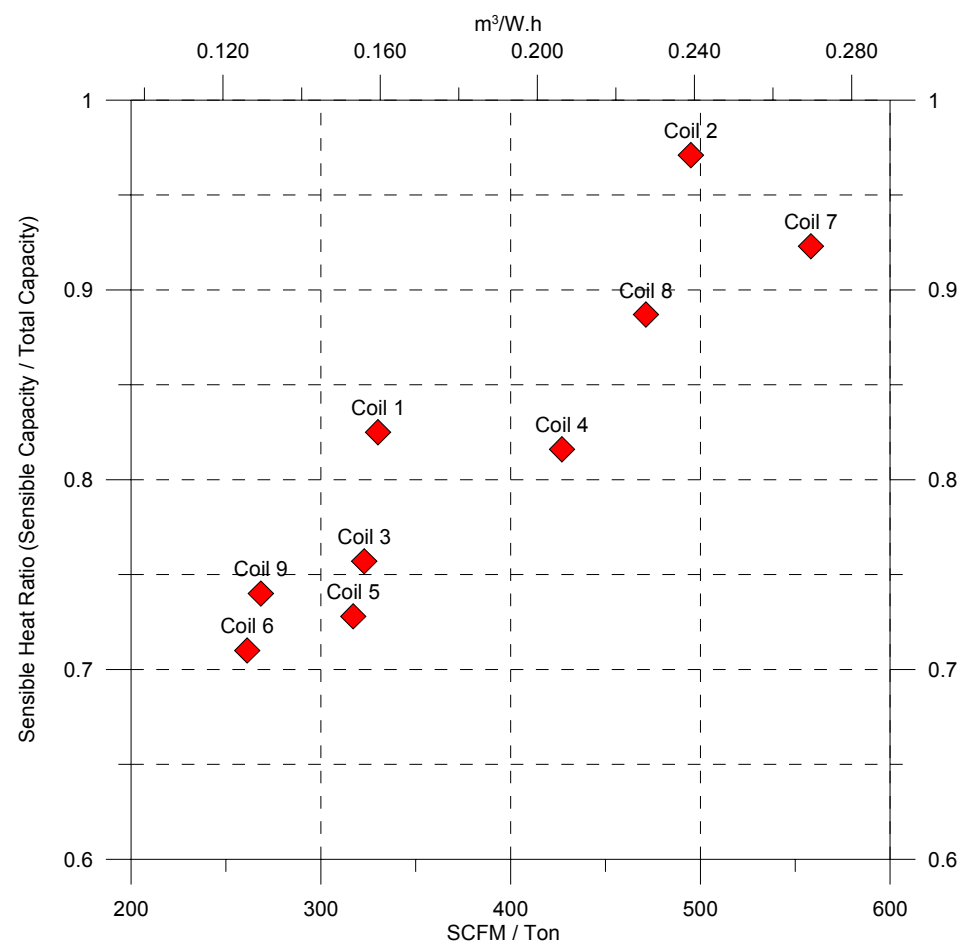

Figure 3.4.12: NIST measured SHR as a function of airflow rate per unit of cooling capacity at an evaporator exit saturation temperature of $16.2^{\circ} \mathrm{C}\left(50.0^{\circ} \mathrm{F}\right)$ 


\section{4: MIXED SYSTEM PERFORMANCE PREDICTION}

\section{1: Calculation of $Q(95), Q(82)$ and $\operatorname{EER}(82)$}

With the coil capacity coefficients from Table 3.4.1 and CD Unit capacity coefficients from Table 4.1.1, the C-B Method was used to calculate cooling capacity and EER at $27.8^{\circ} \mathrm{C}\left(82.0^{\circ} \mathrm{F}\right)$, and cooling capacity at $35.0^{\circ} \mathrm{C}\left(95.0^{\circ} \mathrm{F}\right)$ for all coils.

Table 4.1.1: CD Unit linear coefficients for power and capacity at A-Test and B-Test conditions from ARI

\begin{tabular}{|c|c|c|c|c|c|c|c|c|}
\hline \multirow[b]{2}{*}{ Coil } & \multicolumn{2}{|c|}{$q(82)$} & \multicolumn{2}{|c|}{$p(82)$} & \multicolumn{2}{|c|}{$q(95)$} & \multicolumn{2}{|c|}{$p(95)$} \\
\hline & $\begin{array}{c}\text { Slope } \\
\mathrm{W} /{ }^{\circ} \mathrm{C} \\
\left(\mathrm{Btu} / \mathrm{h}^{\circ} \mathrm{F}\right)\end{array}$ & $\begin{array}{l}\text { Intercept } \\
\text { W (Btu/h) }\end{array}$ & $\begin{array}{l}\text { Slope } \\
W /{ }^{\circ} \mathrm{C} \\
\left(\mathrm{W} /{ }^{\circ} \mathrm{F}\right)\end{array}$ & $\begin{array}{c}\text { Intercept } \\
\text { W }\end{array}$ & $\begin{array}{c}\text { Slope } \\
\text { W/ }{ }^{\circ} \mathrm{C} \\
\left(\mathrm{Btu} / \mathrm{h}^{\circ} \mathrm{F}\right)\end{array}$ & $\begin{array}{l}\text { Intercept } \\
\text { W (Btu/h) }\end{array}$ & $\begin{array}{l}\text { Slope } \\
\text { W/ }{ }^{\circ} \mathrm{C} \\
\left(\mathrm{W} /{ }^{\circ} \mathrm{F}\right)\end{array}$ & $\begin{array}{c}\text { Intercept } \\
\text { W }\end{array}$ \\
\hline 1 & $\begin{array}{c}312.7 \\
(592.7)\end{array}$ & $\begin{array}{c}2092.9 \\
(7141.5)\end{array}$ & $\begin{array}{l}7.38 \\
(4.1)\end{array}$ & 2340.0 & $\begin{array}{c}300.2 \\
(569.1)\end{array}$ & $\begin{array}{c}2217.7 \\
(7567.0)\end{array}$ & $\begin{array}{l}5.76 \\
(3.2)\end{array}$ & 2717.1 \\
\hline 2 & $\begin{array}{c}184.7 \\
(350.2)\end{array}$ & $\begin{array}{c}2979.2 \\
(10165.6)\end{array}$ & $\begin{array}{l}31.14 \\
(17.3)\end{array}$ & 1242.9 & $\begin{array}{c}163.2 \\
(309.3)\end{array}$ & $\begin{array}{c}2785.3 \\
(9503.9)\end{array}$ & $\begin{array}{l}35.64 \\
(19.8)\end{array}$ & 1289.6 \\
\hline 3 & $\begin{array}{c}312.7 \\
(592.7)\end{array}$ & $\begin{array}{c}2092.9 \\
(7141.5)\end{array}$ & $\begin{array}{l}7.38 \\
(4.1)\end{array}$ & 2340.0 & $\begin{array}{c}300.2 \\
(569.1)\end{array}$ & $\begin{array}{c}2217.7 \\
(7567.0)\end{array}$ & $\begin{array}{l}5.76 \\
(3.2)\end{array}$ & 2717.1 \\
\hline $4^{*}$ & $\begin{array}{c}237.1 \\
(449.5)\end{array}$ & $\begin{array}{c}-622.6 \\
(-2124.6)\end{array}$ & $\begin{array}{l}22.14 \\
(12.3)\end{array}$ & 880.9 & $\begin{array}{c}232.2 \\
(440.2)\end{array}$ & $\begin{array}{c}-1253.0 \\
(-4275.5)\end{array}$ & $\begin{array}{c}26.1 \\
(14.5)\end{array}$ & 877.7 \\
\hline 5 & $\begin{array}{c}266.2 \\
(504.7)\end{array}$ & $\begin{array}{c}674.2 \\
(2300.5)\end{array}$ & $\begin{array}{l}32.22 \\
(17.9)\end{array}$ & 1177.1 & $\begin{array}{c}263.3 \\
(499.2)\end{array}$ & $\begin{array}{c}22.7 \\
(77.3)\end{array}$ & $\begin{array}{c}38.7 \\
(21.5)\end{array}$ & 1069.6 \\
\hline 6 & $\begin{array}{c}581.6 \\
(1102.5)\end{array}$ & $\begin{array}{c}3886.6 \\
(13261.7)\end{array}$ & $\begin{array}{l}28.98 \\
(16.1)\end{array}$ & 3584.9 & $\begin{array}{c}564.6 \\
(1070.2)\end{array}$ & $\begin{array}{c}2995.4 \\
(10220.7)\end{array}$ & $\begin{array}{l}32.94 \\
(18.3)\end{array}$ & 4123.4 \\
\hline 7 & $\begin{array}{c}484.1 \\
(917.6)\end{array}$ & $\begin{array}{c}-148.4 \\
(-506.2)\end{array}$ & $\begin{array}{l}31.68 \\
(17.6)\end{array}$ & 2253.8 & $\begin{array}{c}508.6 \\
(964.1) \\
\end{array}$ & $\begin{array}{c}-2569.6 \\
(-8767.8)\end{array}$ & $\begin{array}{l}38.34 \\
(21.3)\end{array}$ & 2278.8 \\
\hline 8 & $\begin{array}{c}96.8 \\
(735.5) \\
\end{array}$ & $\begin{array}{c}3140.6 \\
(10716.2)\end{array}$ & $\begin{array}{c}138.24 \\
(76.8) \\
\end{array}$ & 36.4 & $\begin{array}{l}336.6 \\
(638)\end{array}$ & $\begin{array}{c}3420.9 \\
(11672.7)\end{array}$ & $\begin{array}{c}172.8 \\
(96)\end{array}$ & -697.3 \\
\hline 9 & $\begin{array}{c}286.6 \\
(543.2)\end{array}$ & $\begin{array}{c}984.7 \\
(3359.9)\end{array}$ & $\begin{array}{l}10.8 \\
(6.0)\end{array}$ & 1692.8 & $\begin{array}{c}278.5 \\
(528.0)\end{array}$ & $\begin{array}{c}423.0 \\
(1443.2)\end{array}$ & $\begin{array}{l}11.52 \\
(6.4)\end{array}$ & 1990.9 \\
\hline
\end{tabular}

${ }^{*}$ Coil 4 airflow was too high and results cannot be compared directly to ARI tests

The calculation procedure, which we illustrated graphically in Figure 1.1, was implemented computationally by solving the set of two linear equations for the evaporation temperature at which the cooling capacity of the mixed coil equals the cooling capacity of the CD Unit:

$$
\begin{gathered}
q_{\mathrm{CD}}=\mathrm{B}_{\mathrm{CD}}+\mathrm{A}_{\mathrm{CD}} T_{\text {evap }}=q_{\text {mixed }}=\mathrm{B}_{\text {mixed }}+T_{\text {evap }} \mathrm{A}_{\text {mixed }} \\
T_{\text {evap }}=\frac{\left(\mathrm{B}_{\text {mixed }}-\mathrm{B}_{\mathrm{CD}}\right)}{\left(\mathrm{A}_{\mathrm{CD}}-\mathrm{A}_{\text {mixed }}\right)}
\end{gathered}
$$

In the equations above, B represents the intercept and A represents the slope for the CD Unit (CD subscript) and mixed evaporator (mixed subscript), respectively. Applying the obtained value of the saturation temperature into either capacity equation yields the capacity of the evaporator of the mixed system. The rated cooling capacity of the mixed system was obtained by reducing the evaporator capacity by the fan heat. For coils equipped with a fan, the fan heat was measured; for other coils it was calculated according to ARI Standard 210/240 (ARI 2003).

$$
Q=q+Q_{f a n}
$$


Similarly, the power of the mixed system was obtained by applying the value of the evaporator saturation temperature from equation 4.1.2 into the condensing unit power equation 4.1 .4 and making adjustment for the indoor fan power as shown in equation 4.1.5.

$$
\begin{gathered}
p_{C D}=b_{C D}+a_{C D} T_{\text {evap }} \\
P_{C D}=p_{C D}+P_{\text {fan }}
\end{gathered}
$$

Table 4.1.2 shows the results for A-Test mixed system capacities. Table 4.1.3 presents the results for B-Test capacity, power, and EER.

Table 4.1.2: Mixed system A-Test capacity from the C-B Method

\begin{tabular}{|c|c|c|c|c|c|}
\hline Coil & $\begin{array}{c}T_{\text {evap }} \\
{ }^{\circ} \mathrm{C}\left({ }^{\circ} \mathrm{F}\right)\end{array}$ & $\begin{array}{c}q(95) \\
W \\
(\mathrm{Btu} / \mathrm{h})\end{array}$ & $\begin{array}{c}\text { Indoor } \\
\text { Airflow } \\
\mathrm{m}^{3} / \mathrm{h} \\
(\mathrm{scfm})\end{array}$ & $\begin{array}{c}Q_{\text {fan }}{ }^{(1)} \\
W \\
(\mathrm{Btu} / \mathrm{h})\end{array}$ & $\begin{array}{c}\text { Q(95) } \\
W \\
(\mathrm{Btu} / \mathrm{h})\end{array}$ \\
\hline 1 & $8.1(46.6)$ & $9990(34089)$ & $1699(1000)$ & $366.3(1250.0)$ & $9624(32839)$ \\
\hline 2 & $5.8(42.5)$ & $6639(22653)$ & $1368(805)$ & $294.9(1006.3)$ & $6344(21647)$ \\
\hline 3 & $7.9(46.3)$ & $9935(33898)$ & $1621(954)$ & $349.5(1192.5)$ & $9585(32705)$ \\
\hline $4^{*}$ & $9.9(49.9)$ & $5184(17688)$ & $1342(790)$ & $289.4(987.5)$ & $4894(16700)$ \\
\hline 5 & $9.4(49.0)$ & $7188(24526)$ & $1279(753)$ & $271.0(925.5)$ & $6917(23601)$ \\
\hline 6 & $6.2(43.2)$ & $16541(56439)$ & $1954(1150)$ & $784.0(2677.5)$ & $15756(53761)$ \\
\hline 7 & $4.5(40.2)$ & $8781(29962)$ & $2047(1205)$ & $441.5(1506.3)$ & $8340(28456)$ \\
\hline 8 & $5.9(42.6)$ & $11386(38851)$ & $2360(1389)$ & $507.0(1736.3)$ & $10877(37115)$ \\
\hline 9 & $6.2(43.2)$ & $7114(24274)$ & $849(500)$ & $364.0(1243.1)$ & $6749(23030)$ \\
\hline
\end{tabular}

(1) For units with no fan $Q_{\text {fan }}$ was calculated to be $1250 \mathrm{Btu} / \mathrm{h}$ per $1000 \mathrm{scfm}$ of airflow (ARI 210/240-2003).

${ }^{*}$ Coil 4 airflow was too high and results cannot be compared directly to ARI tests 
Table 4.1.3: Mixed system B-Test capacity, power, and EER from the C-B Method

\begin{tabular}{|c|c|c|c|c|c|c|c|c|}
\hline Coil & $\begin{array}{l}T_{\text {evap }} \\
{ }^{\circ} \mathrm{C}\left({ }^{\circ} \mathrm{F}\right)\end{array}$ & $\begin{array}{c}q(82) \\
W \\
(B+u / h)\end{array}$ & $\begin{array}{c}p_{C D}(82) \\
\mathrm{W}\end{array}$ & $\begin{array}{c}\text { Indoor } \\
\text { Airflow, } \\
\mathrm{m}^{3} / \mathrm{h} \text { (scfm) }\end{array}$ & $\begin{array}{c}Q_{f a n}^{(1)} \\
W \\
(B t u / h)\end{array}$ & $\begin{array}{c}P_{f a n}^{(2)} \\
\mathrm{W}\end{array}$ & $\begin{array}{c}Q(82) \\
W,(B t u / h)\end{array}$ & $\begin{array}{c}\text { EER(82) } \\
\text { W/W } \\
\text { (Btu/W h) }\end{array}$ \\
\hline 1 & $\begin{array}{c}7.93 \\
(46.3) \\
\end{array}$ & $\begin{array}{c}10192 \\
(34563)\end{array}$ & 2530 & $\begin{array}{c}1699 \\
(1000) \\
\end{array}$ & $\begin{array}{c}365.0 \\
(1250.0)\end{array}$ & 365.0 & $\begin{array}{c}9763 \\
(33313)\end{array}$ & $\begin{array}{c}3.37 \\
(11.51)\end{array}$ \\
\hline 2 & $\begin{array}{c}5.01 \\
(41.0) \\
\end{array}$ & $\begin{array}{c}7190 \\
(24532) \\
\end{array}$ & 1953 & $\begin{array}{l}1368 \\
(805) \\
\end{array}$ & $\begin{array}{c}293.8 \\
(1006.3)\end{array}$ & 293.8 & $\begin{array}{c}6895 \\
(23525) \\
\end{array}$ & $\begin{array}{c}3.07 \\
(10.47) \\
\end{array}$ \\
\hline 3 & $\begin{array}{c}7.72 \\
(45.9)\end{array}$ & $\begin{array}{c}10066 \\
(34347)\end{array}$ & 2528 & $\begin{array}{l}1621 \\
(954)\end{array}$ & $\begin{array}{c}348.2 \\
(1192.5)\end{array}$ & 348.2 & $\begin{array}{c}9717 \\
(33155)\end{array}$ & $\begin{array}{c}3.38 \\
(11.53)\end{array}$ \\
\hline $4^{*}$ & $\begin{array}{c}8.88 \\
(48.0)\end{array}$ & $\begin{array}{c}5699 \\
(19445)\end{array}$ & 1471 & $\begin{array}{l}1342 \\
(790)\end{array}$ & $\begin{array}{c}288.4 \\
(987.5) \\
\end{array}$ & 288.4 & $\begin{array}{c}5410 \\
(18458)\end{array}$ & $\begin{array}{c}3.07 \\
(10.49)\end{array}$ \\
\hline 5 & $\begin{array}{c}8.51 \\
(47.3)\end{array}$ & $\begin{array}{c}7666 \\
(26182)\end{array}$ & 2024 & $\begin{array}{l}1279 \\
(753)\end{array}$ & $\begin{array}{c}271 \\
(924.7)\end{array}$ & 271.0 & $\begin{array}{c}7402 \\
(25258)\end{array}$ & $\begin{array}{c}3.27 \\
(11.01)\end{array}$ \\
\hline 6 & $\begin{array}{c}5.42 \\
(41.7)\end{array}$ & $\begin{array}{c}17361 \\
(59291)\end{array}$ & 4257 & $\begin{array}{c}1954 \\
(1150)\end{array}$ & $\begin{array}{c}784 \\
(2675.0)\end{array}$ & 784.0 & $\begin{array}{c}16593 \\
(56616)\end{array}$ & $\begin{array}{c}3.29 \\
(11.23)\end{array}$ \\
\hline 7 & $\begin{array}{c}2.52 \\
(36.5)\end{array}$ & $\begin{array}{c}9677 \\
(33019)\end{array}$ & 2897 & $\begin{array}{c}2047 \\
(1205)\end{array}$ & $\begin{array}{c}439.8 \\
(1506.3)\end{array}$ & 439.8 & $\begin{array}{c}9236 \\
(31513)\end{array}$ & $\begin{array}{c}2.77 \\
(9.44)\end{array}$ \\
\hline 8 & $\begin{array}{c}5.11 \\
(41.1)\end{array}$ & $\begin{array}{c}12009 \\
(40974)\end{array}$ & 3196 & $\begin{array}{c}2360 \\
(1389)\end{array}$ & $\begin{array}{c}507.0 \\
(1736.3)\end{array}$ & 507.0 & $\begin{array}{c}11500 \\
(39238)\end{array}$ & $\begin{array}{c}3.11 \\
(10.6)\end{array}$ \\
\hline 9 & $\begin{array}{c}5.37 \\
(41.7)\end{array}$ & $\begin{array}{c}7610 \\
(25991)\end{array}$ & 1943 & $\begin{array}{l}849 \\
(500)\end{array}$ & $\begin{array}{c}364 \\
(1241.9)\end{array}$ & 364.0 & $\begin{array}{c}7253 \\
(24749)\end{array}$ & $\begin{array}{c}3.14 \\
(10.73)\end{array}$ \\
\hline
\end{tabular}

(1) For units with no fan $Q_{\text {fan }}$ is calculated to be 1250 Btu/h per $1000 \mathrm{scfm}$ of airflow (ARI 210/240-2003).

(2) For units with no fan $P_{\text {fan }}$ is calculated to be $365 \mathrm{~W}$ per $1000 \mathrm{scfm}$ of airflow (ARI 210/240-2003).

Coil 4 airflow was too high and results cannot be compared directly to ARI tests.

\section{2: Calculation of SEER}

The calculation of SEER involves the value of $\operatorname{EER}(82)$ and the cyclic degradation coefficient $\mathrm{C}_{\mathrm{D}}$.

$$
\operatorname{SEER}=\left(1-0.5 \cdot C_{D}\right) \operatorname{EER}(82)
$$

For the tested systems, $C_{D}$ was typically obtained by conducting dry-coil steady-state and cyclic tests (C and D tests of ARI Standard 210/240, (ARI 2003)). Alternatively, the rater may use the 0.25 default value instead of performing the tests. In practice, $C_{D}$ values fall between 0.0 and 0.25 . Since, by definition, the mixed system is not tested, the $C_{D}$ value must be obtained from some engineering analysis or the default value of 0.25 must be taken.

Dougherty (2004), working with DOE and ARI, performed a statistical analysis of experimentally determined $C_{D}$ values for a large sample of systems. He grouped the studied systems into four basic categories shown in Table 4.2.1. The analysis of $C_{D}$ values for these four system categories produced the $C_{D}$ percentiles shown in Table 4.2.2. 
Table 4.2.1: System classifications for cyclic degradation coefficient analysis

\begin{tabular}{|c|c|c|c|}
\hline $\begin{array}{c}\text { System } \\
\text { Category }\end{array}$ & $\begin{array}{c}\text { Equalize During Off } \\
\text { Cycle }\end{array}$ & Indoor Fan Off Delay & $\begin{array}{c}\text { System } \\
\text { Components }\end{array}$ \\
\hline \hline A & Yes & No & $\begin{array}{c}\text { Cap Tube } \\
\text { Orifice } \\
\text { Bleed TXV }\end{array}$ \\
\hline B1 & No & No & $\begin{array}{c}\text { Non-Bleed TXV } \\
\text { Electronic Expansion Device } \\
\text { Liquid Line Solenoid }\end{array}$ \\
\hline B2 & Yes & Yes & $\begin{array}{c}\text { Cap Tube } \\
\text { Orifice } \\
\text { Bleed TXV }\end{array}$ \\
\hline \hline C & No & Yes & $\begin{array}{r}\text { Non-Bleed TXV } \\
\text { Electronic Expansion Device } \\
\text { Liquid Line Solenoid }\end{array}$ \\
\hline
\end{tabular}

The range of $C_{D}$ values is rather significant in each equipment category. In category $A$, for example, the classification for all nine mixed systems tested for this project, the difference between the $50^{\text {th }}$ and $99^{\text {th }}$ percentiles is $0.24-0.09=0.15$. This means that assuming the $50^{\text {th }}$ percentile value for the mixed system with an actual $C_{D}$ of 0.24 will result in a SEER prediction error of $7.5 \%$. For illustration in Table 4.2.3, we generated two SEER numbers for mixed systems using $C_{D}$ values of 0.22 and 0.24 , which correspond to the $95^{\text {th }}$ and $99^{\text {th }}$ percentiles, respectively. While both choices are conservative statistically, they still represent a risk of SEER overprediction by $1.5 \%$ and $0.5 \%$, respectively, should the actual $C_{D}$ value be the maximum of 0.25 . We believe that the most accurate assignment of $C_{D}$ for the mixed system would be that of the matched system if the changes implemented in the mixed system do not move it to a different equipment category, as defined in Table 4.2.1.

Table 4.2.2: Cyclic degradation coefficient values for different system categories

\begin{tabular}{|c|c|c|c|c|}
\hline Percentile & A & B1 & B2 & C \\
\hline $99^{\text {th }}$ & 0.24 & 0.16 & 0.22 & 0.15 \\
\hline $95^{\text {th }}$ & 0.22 & 0.14 & 0.14 & 0.12 \\
\hline $90^{\text {th }}$ & 0.16 & 0.14 & 0.12 & 0.10 \\
\hline $85^{\text {th }}$ & 0.14 & 0.12 & 0.11 & 0.09 \\
\hline $80^{\text {th }}$ & 0.12 & 0.12 & 0.10 & 0.08 \\
\hline $75^{\text {th }}$ & 0.12 & 0.11 & 0.10 & 0.07 \\
\hline $70^{\text {th }}$ & 0.11 & 0.11 & 0.09 & 0.06 \\
\hline $60^{\text {th }}$ & 0.10 & 0.9 & 0.08 & 0.05 \\
\hline $50^{\text {th }}$ & 0.09 & 0.07 & 0.07 & 0.04 \\
\hline Sample Size & & & & \\
\hline
\end{tabular}


Table 4.2.3: Mixed system SEERs calculated using statistically determined $C_{D}$ for $95^{\text {th }}$ and $99^{\text {th }}$ percentiles

\begin{tabular}{|c|c|c|c|c|}
\hline \multirow{2}{*}{ Coil } & \multicolumn{2}{|c|}{$\begin{array}{c}\text { Cyclic Degradation } \\
\text { Coefficient, } \mathrm{C}_{\mathrm{d}}\end{array}$} & \multicolumn{2}{c|}{ SEER $(\mathrm{Btu} / \mathrm{W} \cdot \mathrm{h})$} \\
\cline { 2 - 5 } & $95^{\text {th }}$ & $99^{\text {th }}$ & $95^{\text {th }}$ & $99^{\text {th }}$ \\
\hline 1 & 0.22 & 0.24 & 10.24 & 10.13 \\
\hline 2 & 0.22 & 0.24 & 9.32 & 9.22 \\
\hline 3 & 0.22 & 0.24 & 10.26 & 10.14 \\
\hline $4^{*}$ & 0.22 & 0.24 & 9.34 & 9.23 \\
\hline 5 & 0.22 & 0.24 & 10.08 & 9.97 \\
\hline 6 & 0.22 & 0.24 & 10.33 & 10.21 \\
\hline 7 & 0.22 & 0.24 & 8.41 & 8.31 \\
\hline 8 & 0.22 & 0.24 & 9.43 & 9.32 \\
\hline 9 & 0.22 & 0.24 & 9.89 & 9.78 \\
\hline
\end{tabular}

Coil 4 airflow was too high and results cannot be compared directly to ARI tests.

\section{5: NIST PREDICTIONS AND ARI DATABASE COMPARISON}

\section{1: A-Test capacity comparison}

Table 5.1.1 and Figure 5.1.1 present mixed system capacities, Q(95), from the C-B Method and the ARI database for the A-Test conditions. Capacity predictions from the C-B Method were within $\pm 5 \%$ of the ARI tests for five of eight coils. Among the three cases with poor predictions, the disagreement was as high as $17.6 \%$.

Table 5.1.1: Mixed system A-Test capacities from ARI Tests and C-B Method

\begin{tabular}{|c|c|c|c|c|c|c|c|c|}
\hline Coil & $\begin{array}{c}\text { C-B } \\
\text { Method } \\
T_{\text {evap }} \\
{ }^{\circ} \mathrm{C}\left({ }^{\circ} \mathrm{F}\right)\end{array}$ & $\begin{array}{c}\text { ARI } \\
\text { Test } \\
T_{\text {evap }} \\
{ }^{\circ} \mathrm{C}\left({ }^{\circ} \mathrm{F}\right)\end{array}$ & $\begin{array}{c}\text { C-B } \\
\text { Method } \\
q(95) \\
\text { W } \\
(\text { Btu/h) }\end{array}$ & $\begin{array}{c}\text { Indoor } \\
\text { Airflow } \\
\mathrm{m}^{3} / \mathrm{h} \\
\text { (scfm) }\end{array}$ & $\begin{array}{c}Q_{\mathrm{fan}} \\
W \\
(\mathrm{Btu} / \mathrm{h})\end{array}$ & $\begin{array}{c}\text { ARI Test } \\
\text { Q(95) } \\
\text { W } \\
\text { (Btu/h) }\end{array}$ & $\begin{array}{c}\text { C-B } \\
\text { Method } \\
Q(95) \\
W \\
\text { (Btu/h) }\end{array}$ & $\begin{array}{c}Q_{\text {diff }} \\
100 \%(N I S T- \\
\text { ARI)/ARI }\end{array}$ \\
\hline 1 & $\begin{array}{c}8.1 \\
(46.6)\end{array}$ & $\begin{array}{c}7.2 \\
(45.0)\end{array}$ & $\begin{array}{c}9991 \\
(34089)\end{array}$ & $\begin{array}{c}1699 \\
(1000)\end{array}$ & $\begin{array}{c}366.3^{(1)} \\
(1250.0)\end{array}$ & $\begin{array}{c}10034 \\
(34238)\end{array}$ & $\begin{array}{c}9624 \\
(32839)\end{array}$ & -4.1 \\
\hline 2 & $\begin{array}{c}5.8 \\
(42.5)\end{array}$ & $\begin{array}{c}6.1 \\
(43.0)\end{array}$ & $\begin{array}{c}6639 \\
(22653)\end{array}$ & $\begin{array}{l}1368 \\
(805)\end{array}$ & $\begin{array}{l}294.9^{(1)} \\
(1006.3)\end{array}$ & $\begin{array}{c}6529 \\
(22278)\end{array}$ & $\begin{array}{c}6344 \\
(21647)\end{array}$ & -2.8 \\
\hline 3 & $\begin{array}{c}7.9 \\
(46.3)\end{array}$ & $\begin{array}{c}7.6 \\
(45.6)\end{array}$ & $\begin{array}{c}9935 \\
(33898)\end{array}$ & $\begin{array}{l}1621 \\
(954)\end{array}$ & $\begin{array}{c}349.5^{(1)} \\
(1192.5)\end{array}$ & $\begin{array}{c}9609 \\
(32786)\end{array}$ & $\begin{array}{c}9585 \\
(32705)\end{array}$ & -0.2 \\
\hline $4^{*}$ & $\begin{array}{c}9.9 \\
(49.9)\end{array}$ & $\begin{array}{c}8.4 \\
(47.2)\end{array}$ & $\begin{array}{c}5184 \\
(17688)\end{array}$ & $\begin{array}{l}1342 \\
(790)\end{array}$ & $\begin{array}{l}289.4^{(1)} \\
(987.5)\end{array}$ & $\begin{array}{c}5073 \\
(17311)\end{array}$ & $\begin{array}{c}4894 \\
(16700)\end{array}$ & -3.5 \\
\hline 5 & $\begin{array}{c}9.4 \\
(49.0)\end{array}$ & $\begin{array}{c}10.5 \\
(50.9)\end{array}$ & $\begin{array}{c}7188 \\
(24526)\end{array}$ & $\begin{array}{l}1279 \\
(753)\end{array}$ & $\begin{array}{l}271.0^{(1)} \\
(925.5)\end{array}$ & $\begin{array}{c}6787 \\
(23157)\end{array}$ & $\begin{array}{c}6917 \\
(23601)\end{array}$ & 1.9 \\
\hline 6 & $\begin{array}{c}6.2 \\
(43.2)\end{array}$ & $\begin{array}{c}7.2 \\
(44.9)\end{array}$ & $\begin{array}{c}16541 \\
(56439)\end{array}$ & $\begin{array}{c}1954 \\
(1150)\end{array}$ & $\begin{array}{c}784.0^{(2)} \\
(2677.5)\end{array}$ & $\begin{array}{c}14407 \\
(49159)\end{array}$ & $\begin{array}{c}15756 \\
(53761)\end{array}$ & 9.4 \\
\hline 7 & $\begin{array}{c}4.5 \\
(40.2)\end{array}$ & $\begin{array}{c}6.8 \\
(44.3)\end{array}$ & $\begin{array}{c}8781 \\
(29962)\end{array}$ & $\begin{array}{c}2047 \\
(1205)\end{array}$ & $\begin{array}{c}441.5^{(2)} \\
(1506.3)\end{array}$ & $\begin{array}{c}10122 \\
(34537)\end{array}$ & $\begin{array}{c}8340 \\
(28456)\end{array}$ & -17.6 \\
\hline 8 & $\begin{array}{c}5.9 \\
(42.6)\end{array}$ & $\begin{array}{c}5.7 \\
(42.2)\end{array}$ & $\begin{array}{c}11387 \\
(38851)\end{array}$ & $\begin{array}{c}2360 \\
(1389)\end{array}$ & $\begin{array}{c}507.0^{(1)} \\
(1736.3)\end{array}$ & $\begin{array}{c}12591 \\
(42963)\end{array}$ & $\begin{array}{c}10878 \\
(37115)\end{array}$ & -13.6 \\
\hline 9 & $\begin{array}{c}6.2 \\
(43.2)\end{array}$ & $\begin{array}{c}4.1 \\
(44.2)\end{array}$ & $\begin{array}{c}7114 \\
(24274)\end{array}$ & $\begin{array}{c}849 \\
(500)\end{array}$ & $\begin{array}{c}364^{(2)} \\
(1243.1)\end{array}$ & $\begin{array}{c}6480 \\
(22112)\end{array}$ & $\begin{array}{c}6749 \\
(23030)\end{array}$ & 4.2 \\
\hline
\end{tabular}

(1) Coil without a fan; $Q_{f a n}$ was calculated to be $1250 \mathrm{Btu} / \mathrm{h}$ per $1000 \mathrm{scfm}$ of airflow (ARI 210/240-2003)

(2)

Coil equipped with a fan; fan power measured by NIST

${ }^{*}$ Coil 4 airflow was too high and results cannot be compared directly to ARI tests. 


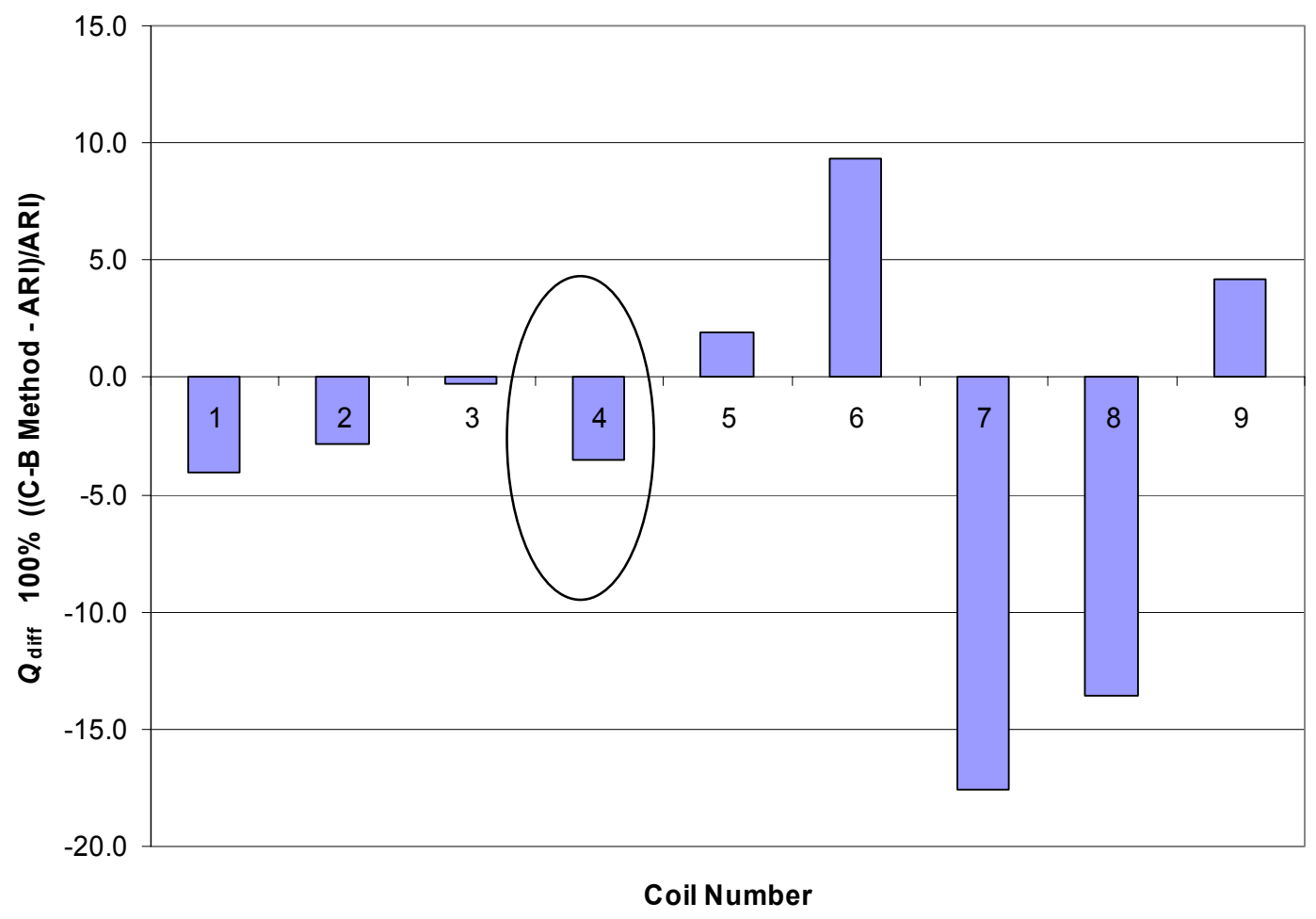

Figure 5.1.1: Comparison of mixed system A-test capacities from the ARI tests and the C-B Method

(Note: Coil 4 airflow was too high and results cannot be compared directly to ARI tests)

Table 5.1.1 also shows evaporator saturation temperatures obtained from the C-B Method and measured during mixed system certification tests. Ideally, these temperatures should be the same or very close for good capacity predictions. One can rationalize that a saturation temperature from the C-B Method that is lower than that from the certification tests should result in an overprediction of capacity while a higher C-B Method saturation temperature should drive toward the opposite effect. This physical rationale holds somewhat for Coils 1, 5, and 6, while other coils do not conform. In particular, Coil 7, associated with the largest underprediction of $\mathrm{Q}(95)$ of $17.6 \%$ has a $4.5^{\circ} \mathrm{C}\left(40.2{ }^{\circ} \mathrm{F}\right) \mathrm{C}-\mathrm{B}$ Method saturation temperature versus a $6.8^{\circ} \mathrm{C}$ $\left(44.3^{\circ} \mathrm{F}\right)$ saturation temperature measured during certification tests.

To further explore the reasons for inconsistent $Q(95)$ predictions, we compared test-obtained mixed system $Q(95)$ capacities with those calculated for mixed evaporators from NISTdeveloped capacity lines and those calculated for condensing units from condensing performance curves using the same evaporator saturation temperature as measured during the mixed system certification tests. Table 5.1.2 presents the data. It is desirable for these three capacities to be close, ideally equal, to each other. The table shows that NIST-calculated capacities agreed with the certification test capacities for Coils $1,3,6$, and 9 within $\pm 2.5 \%$, which is a remarkable agreement. The capacities for coils 2 and 5 are underpredicted within $6.5 \%$. The largest disagreement of $-27.6 \%$ is for Coil 7 . For condensing unit capacities, the 
agreement with mixed system data was within $\pm 6.5 \%$ for six cases. In the remaining cases, the deviations from mixed system capacities were as much as $-14.2 \%$.

Looking back at the predicted $Q(95)$ presented in Table 5.1.1, we can see that we obtained good predictions of $Q(95)$ in every case where the capacities from three sources shown in Table 5.1 .2 are in good agreement (Coils 1, 2, 3, and 9). Also good $Q(95)$ predictions are for Coil 5 in Table 5.1.1; in this case the capacity calculated from evaporator and condensing unit correlations in Table 5.1.2 underpredicted and overpredicted the mixed system capacity by a similar percentage. The result is a good prediction of $Q(95)$ at a somewhat different evaporator saturation temperature than that measured during a system test.

In the cases with the largest $Q(95)$ prediction errors, Coils 6, 7 and 8, no offsetting of errors took place; even for Coil 8 where the C-B Method predicted the evaporator saturation temperature within $0.2{ }^{\circ} \mathrm{C}\left(0.4^{\circ} \mathrm{F}\right)$. Since evaporator capacity and mixed system capacity for Coil 6 in Table 5.1 .2 agree within $2.5 \%$ while the condensing unit capacity deviates by $13.1 \%$, a suggestion can be made that the condensing unit correlation could be faulted for the $Q(95)$ underprediction. Using the same rationale, a case against the evaporator capacity correlation could be made for Coil 7. For Coil 8, the evaporator and condensing unit correlations yield similar capacities and disagree with the system test data by a similar capacity percentage, $-12.2 \%$ and $-14.2 \%$, respectively, suggesting some testing irregularity or an error in data handling.

Table 5.1.3 allows additional analysis of capacity predictions from the NIST-developed capacity lines. The table shows the tested mixed system capacities and those calculated using capacity lines (adjusted for the indoor fan heat) as presented in Table 5.1.2, but it includes temperature and subcooling of the refrigerant entering the expansion valve and the refrigerant superheat at the evaporator exit. Since values of these parameters are different for most cases, it was of interest to assess the extent to which these differences could affect the evaporator capacity predictions. For this purpose, we used the EVAP-COND simulation package (NIST 2003) to simulate coil performance at different inlet refrigerant temperatures and outlet superheats.

For example, for Coil 7 , the ARI superheat was $2.4^{\circ} \mathrm{C}\left(4.3^{\circ} \mathrm{F}\right)$ and the NIST superheat was $7.3^{\circ} \mathrm{C}\left(13.2^{\circ} \mathrm{F}\right)$; a difference of $4.9^{\circ} \mathrm{C}\left(8.9^{\circ} \mathrm{F}\right)$. Keeping the same evaporator saturation temperature and liquid temperature (inlet quality), and changing the superheat to the ARI value increased the predicted Coil 7 capacity by $494 \mathrm{~W}$ (1686 Btu/h) to $7818 \mathrm{~W}$ (26 $675 \mathrm{Btu} / \mathrm{h})$, thus reducing the percent difference from $-27.6 \%$ to $-22.8 \%$. Changing the liquid temperature from $40.6^{\circ} \mathrm{C}\left(105.1^{\circ} \mathrm{F}\right)$ to the $\mathrm{ARI}$ value of $49.4^{\circ} \mathrm{C}\left(120.9^{\circ} \mathrm{F}\right)$ and keeping the same subcooling of $7.7^{\circ} \mathrm{C}\left(13.8^{\circ} \mathrm{F}\right)$, increased the capacity by an additional $222 \mathrm{~W}(758 \mathrm{Btu} / \mathrm{h})$ to $8038 \mathrm{~W}$ (27 $433 \mathrm{Btu} / \mathrm{h}$ ), thus reducing the ARI-NIST percent difference to $-20.5 \%$. It appears that some installation and test condition related factors are responsible for the remaining $-20.5 \%$ deviation in the Coil 7 results. 
Table 5.1.2: Mixed system A-Test capacities from the ARI tests, condensing unit capacities from CD Unit curves, and evaporator capacities from NIST-developed evaporator capacity curves at the ARI-test evaporating temperature ${ }^{(1,2)}$

\begin{tabular}{|c|c|c|c|c|c|c|c|c|c|c|c|c|c|c|c|c|c|c|}
\hline & \multicolumn{2}{|c|}{ Coil 1} & \multicolumn{2}{|c|}{ Coil 2} & \multicolumn{2}{|c|}{ Coil 3} & \multicolumn{2}{|c|}{ Coil 4* } & \multicolumn{2}{|c|}{ Coil 5} & \multicolumn{2}{|c|}{ Coil 6} & \multicolumn{2}{|c|}{ Coil 7} & \multicolumn{2}{|c|}{ Coil 8} & \multicolumn{2}{|c|}{ Coil 9} \\
\hline & W & Btu/h & W & Btu/h & W & Btu/h & W & Btu/h & W & Btu/h & W & Btu/h & W & Btu/h & W & Btu/h & W & Btu/h \\
\hline ARI Value $Q(95)$ & 10034 & 34238 & 6529 & 22278 & 9609 & 32786 & 5073 & 17311 & 6787 & 23157 & 14407 & 49159 & 10122 & 34537 & 12591 & 42963 & 6480 & 22112 \\
\hline NIST Q(95) & 10282 & 35083 & 6163 & 21029 & 9829 & 33536 & 5622 & 19183 & 6353 & 21679 & 14760 & 50363 & 7324 & 24989 & 11049 & 37702 & 6443 & 21984 \\
\hline CD Unit $Q(95)$ & 10338 & 35273 & 6388 & 21796 & 9475 & 32330 & 4548 & 15518 & 7198 & 24562 & 16294 & 55598 & 9508 & 32441 & 10804 & 36866 & 6899 & 23539 \\
\hline NIST/ARI \% & \multicolumn{2}{|c|}{2.5} & \multicolumn{2}{|c|}{-5.6} & \multicolumn{2}{|c|}{2.3} & \multicolumn{2}{|c|}{10.8} & \multicolumn{2}{|c|}{-6.4} & \multicolumn{2}{|c|}{2.5} & \multicolumn{2}{|c|}{-27.6} & \multicolumn{2}{|c|}{-12.2} & \multicolumn{2}{|c|}{-0.6} \\
\hline CD Unit/ARI \% & \multicolumn{2}{|c|}{3.0} & \multicolumn{2}{|c|}{-2.2} & \multicolumn{2}{|c|}{-1.4} & \multicolumn{2}{|c|}{-10.4} & \multicolumn{2}{|c|}{6.1} & \multicolumn{2}{|c|}{13.1} & \multicolumn{2}{|c|}{-6.1} & \multicolumn{2}{|c|}{-14.2} & \multicolumn{2}{|c|}{6.5} \\
\hline
\end{tabular}

${ }^{(1)}$ Mixed system evaporating temperature, $T_{\text {evap }}$, is listed in Tables 5.1.1.

(2) Indoor fan heat from the mixed system accounted for in all capacity calculations

${ }^{(3)} \mathrm{Q}_{\text {diff }}=100 \%$ (NIST - ARI)/ARI

${ }^{(4)} Q_{\text {diff }}=100 \%(C D$ Unit - ARI)/ARI

${ }^{*}$ Coil 4 airflow was too high and results cannot be compared directly to ARI tests

Table 5.1.3: Mixed system A-test capacities from ARI values and evaporator capacities from NIST-developed evaporator capacity curves at the ARI-test evaporating temperature ${ }^{(1)}$

\begin{tabular}{|c|c|c|c|c|c|c|c|c|c|c|c|c|c|c|c|c|c|c|}
\hline & \multicolumn{2}{|c|}{ Coil 1} & \multicolumn{2}{|c|}{ Coil 2} & \multicolumn{2}{|c|}{ Coil 3} & \multicolumn{2}{|c|}{ Coil 4* } & \multicolumn{2}{|c|}{ Coil 5} & \multicolumn{2}{|c|}{ Coil 6} & \multicolumn{2}{|c|}{ Coil 7} & \multicolumn{2}{|c|}{ Coil 8} & \multicolumn{2}{|c|}{ Coil 9} \\
\hline & ARI & NIST & ARI & NIST & ARI & NIST & ARI & NIST & ARI & NIST & ARI & NIST & ARI & NIST & ARI & NIST & ARI & NIST \\
\hline $\begin{array}{c}T_{\text {liq }}{ }^{\circ} \mathrm{C} \\
\left({ }^{\circ} \mathrm{F}\right)\end{array}$ & $\begin{array}{c}37.2 \\
(99.0)\end{array}$ & $\begin{array}{c}40.7 \\
(105.3)\end{array}$ & $\begin{array}{c}41.1 \\
(106.0)\end{array}$ & $\begin{array}{c}40.5 \\
(104.9)\end{array}$ & $\begin{array}{c}35.2 \\
(95.4)\end{array}$ & $\begin{array}{c}40.5 \\
(104.9)\end{array}$ & $\begin{array}{c}42.2 \\
(108.0)\end{array}$ & $\begin{array}{c}40.7 \\
(105.3)\end{array}$ & $\begin{array}{c}40.3 \\
(104.6)\end{array}$ & $\begin{array}{c}40.4 \\
(104.8)\end{array}$ & $\begin{array}{c}40.3 \\
(104.6)\end{array}$ & $\begin{array}{c}40.4 \\
(104.8)\end{array}$ & $\begin{array}{c}49.4 \\
(120.9)\end{array}$ & $\begin{array}{c}40.6 \\
(105.1)\end{array}$ & $\begin{array}{c}39.1 \\
(102.4)\end{array}$ & $\begin{array}{c}40.9 \\
(105.7)\end{array}$ & $\begin{array}{c}40.8 \\
(105.4)\end{array}$ & $\begin{array}{c}40.6 \\
(105.0)\end{array}$ \\
\hline $\begin{array}{c}T_{\text {sub }}{ }^{\circ} \mathrm{C} \\
\left({ }^{\circ} \mathrm{F}\right)\end{array}$ & $\begin{array}{c}6.9 \\
(12.5)\end{array}$ & $\begin{array}{c}6.2 \\
(11.1)\end{array}$ & $\begin{array}{c}11.8 \\
(21.2)\end{array}$ & $\begin{array}{c}6.6 \\
(11.9)\end{array}$ & $\begin{array}{c}7.7 \\
(13.8)\end{array}$ & $\begin{array}{c}7.7 \\
(13.9)\end{array}$ & $\begin{array}{c}13.8 \\
(24.9)\end{array}$ & $\begin{array}{c}7.6 \\
(13.6)\end{array}$ & $\begin{array}{c}7.7 \\
(13.9)\end{array}$ & $\begin{array}{c}7.7 \\
(13.9)\end{array}$ & $\begin{array}{c}6.4 \\
(11.6)\end{array}$ & $\begin{array}{c}7.7 \\
(13.9)\end{array}$ & $\begin{array}{c}6.7 \\
(12.0)\end{array}$ & $\begin{array}{c}7.7 \\
(13.8)\end{array}$ & $\begin{array}{c}11.1 \\
(19.94)\end{array}$ & $\begin{array}{c}7.1 \\
(12.8)\end{array}$ & $\begin{array}{c}5.9 \\
(10.6)\end{array}$ & $\begin{array}{c}7.6 \\
(13.6)\end{array}$ \\
\hline $\begin{array}{c}T_{\text {evap }}{ }^{\circ} \mathrm{C} \\
\left({ }^{\circ} \mathrm{F}\right)\end{array}$ & $\begin{array}{c}7.2 \\
(45.0)\end{array}$ & $\begin{array}{c}7.2 \\
(45.0)\end{array}$ & $\begin{array}{c}6.1 \\
(43.0)\end{array}$ & $\begin{array}{c}6.1 \\
(43.0)\end{array}$ & $\begin{array}{c}7.6 \\
(45.6)\end{array}$ & $\begin{array}{c}7.6 \\
(45.6)\end{array}$ & $\begin{array}{c}8.4 \\
(47.2)\end{array}$ & $\begin{array}{c}8.4 \\
(47.2)\end{array}$ & $\begin{array}{c}10.5 \\
(50.9)\end{array}$ & $\begin{array}{c}10.5 \\
(50.9)\end{array}$ & $\begin{array}{c}7.2 \\
(44.9)\end{array}$ & $\begin{array}{c}7.2 \\
(44.9)\end{array}$ & $\begin{array}{c}6.8 \\
(44.3)\end{array}$ & $\begin{array}{c}6.8 \\
(44.3)\end{array}$ & $\begin{array}{c}5.7 \\
(42.2)\end{array}$ & $\begin{array}{c}5.7 \\
(42.2)\end{array}$ & $\begin{array}{c}6.8 \\
(44.2)\end{array}$ & $\begin{array}{c}6.8 \\
(44.2)\end{array}$ \\
\hline $\begin{array}{c}T_{\text {suph }}{ }^{\circ} \mathrm{C} \\
\left({ }^{\circ} \mathrm{F}\right)\end{array}$ & $\begin{array}{c}6.2 \\
(11.2)\end{array}$ & $\begin{array}{c}7.1 \\
(12.8)\end{array}$ & $\begin{array}{c}4.7 \\
(8.5)\end{array}$ & $\begin{array}{c}8.1 \\
(14.6)\end{array}$ & $\begin{array}{c}11.6 \\
(20.9)\end{array}$ & $\begin{array}{c}7.4 \\
(13.4)\end{array}$ & $\begin{array}{c}2.1 \\
(3.8)\end{array}$ & $\begin{array}{c}7.4 \\
(13.3)\end{array}$ & $\begin{array}{c}0.8 \\
(1.5)\end{array}$ & $\begin{array}{c}7.8 \\
(14.0)\end{array}$ & $\begin{array}{c}7.1 \\
(12.7)\end{array}$ & $\begin{array}{c}7.6 \\
(13.6)\end{array}$ & $\begin{array}{c}2.4 \\
(4.3)\end{array}$ & $\begin{array}{c}7.3 \\
(13.2)\end{array}$ & $\begin{array}{c}3.9 \\
(7.1)\end{array}$ & $\begin{array}{c}7.4 \\
(13.4)\end{array}$ & $\begin{array}{c}6.2 \\
(11.2)\end{array}$ & $\begin{array}{c}7.8 \\
(14.0)\end{array}$ \\
\hline $\begin{array}{c}Q, W \\
(B t u / h)\end{array}$ & $\begin{array}{c}10034 \\
(34238)\end{array}$ & $\begin{array}{c}10282 \\
(35083)\end{array}$ & $\begin{array}{c}6529 \\
(22278)\end{array}$ & $\begin{array}{c}6163 \\
(21029)\end{array}$ & $\begin{array}{c}9609 \\
(32786)\end{array}$ & $\begin{array}{c}9829 \\
(33536)\end{array}$ & $\begin{array}{c}5073 \\
(17311)\end{array}$ & $\begin{array}{c}5622 \\
(19183)\end{array}$ & $\begin{array}{c}6787 \\
(23157)\end{array}$ & $\begin{array}{c}6353 \\
(21679)\end{array}$ & $\begin{array}{c}14407 \\
(49159)\end{array}$ & $\begin{array}{c}14760 \\
(50363)\end{array}$ & $\begin{array}{c}10122 \\
(34537)\end{array}$ & $\begin{array}{c}7324 \\
(24989)\end{array}$ & $\begin{array}{c}12591 \\
(42963)\end{array}$ & $\begin{array}{c}11049 \\
(37702)\end{array}$ & $\begin{array}{c}6480 \\
(22112)\end{array}$ & $\begin{array}{c}6443 \\
(21984)\end{array}$ \\
\hline$Q_{\text {diff }}(\%)^{(2)}$ & & 2.5 & & -5.6 & & 2.3 & & 10.8 & & -6.4 & & 2.5 & & -27.6 & & -12.2 & & -0.6 \\
\hline
\end{tabular}

(2) Indoor fan heat from the mixed system accounted for in all capacity calculations

${ }^{*}$ Coil 4 airflow was too high and results cannot be compared directly to ARI tests 


\section{2: B-Test EER comparison}

Table 5.2.1 shows EER values from the ARI tests and the C-B Method. Coils 5 and 7 show an agreement within $\pm 5 \%$. The C-B Method overpredicted the ARI values by more than $5 \%$ in four cases. System power, fan power, $Q(82)$, and EER(82) were compared in Figure 5.2.1 to illustrate the sources of disagreement between the C-B Method and the ARI tests.

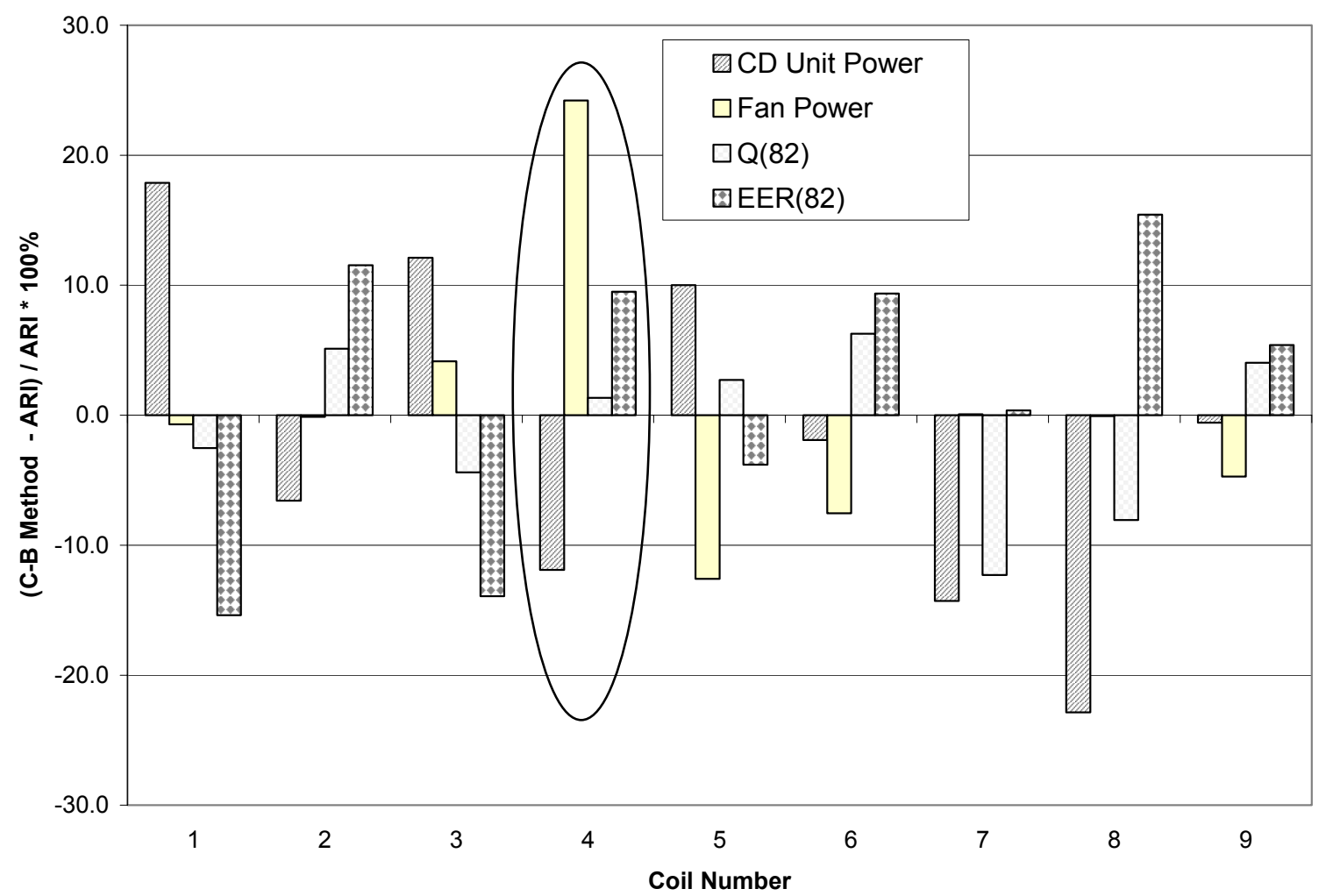

Figure 5.2.1: Percent differences between ARI Values and the C-B Method for the EER(82) calculation (Note: Coil 4 airflow was too high and results cannot be compared directly to ARI tests)

We may note that the largest EER disagreement was a $15.4 \%$ underprediction and a $15.4 \%$ over prediction for Coil 1 and Coil 8, respectively. For Coil 1 the $17.9 \%$ higher power and the $2.5 \%$ lower capacity caused the C-B Method EER(82) to be $15.4 \%$ lower than the ARI Test EER(82). Since the evaporator saturation temperatures were equal for this coil, the $400 \mathrm{~W}$ difference in ARI Test and CD Unit linear fit power is the main contributor to the EER(82) difference. If the ARI Test CD Unit power were used in the C-B Method calculation of $\operatorname{EER}(82)$, the percent difference in $\operatorname{EER}(82)$ would only be $-2.4 \%$.

Coil 8 had a $22.9 \%$ under prediction in CD Unit power compared to the ARI Test results. The C-B Method's lower power was not completely mitigated by a lower capacity, resulting in the $\operatorname{EER}(82)$ being $15.4 \%$ higher than the ARI Test EER(82). The lower evaporating temperature for the C-B Method would tend to produce a higher capacity than the ARI Test capacity, but this was not the case. According to the CD Unit capacity linear fit, a $1.3^{\circ} \mathrm{C}$ $\left(2.4^{\circ} \mathrm{F}\right)$ lower evaporating temperature should increase capacity by $517 \mathrm{~W}(1765 \mathrm{Btu} / \mathrm{h})$. 
This does not equal the $1518 \mathrm{~W}(5179 \mathrm{Btu} / \mathrm{h})$ difference between the C-B Method and ARI Test capacities. If the ARI Test value of power were used with the C-B Method capacity, the resulting $\operatorname{EER}(82)$ percent difference would be $-8.1 \%$.

The C-B Method produced a $13.9 \%$ underprediction of EER(82) for Coil 3. The ARI Test and C-B Method evaporating temperature were within $3.6 \%$. The CD Unit power and the ARI Test power differed by $12.1 \%$. The higher C-B Method power and the lower C-B Method capacity produced the $13.9 \%$ lower EER(82). If the ARI Test power were used with the C-B Method capacity, the EER(82) difference would be $-4.9 \%$.

From examining Table 5.2.1, the clear factor in $\operatorname{EER}(82)$ disagreement is the system power difference between actual tests and the power fits. This is true for all cases except Coil 7. For Coil 7, the evaporating temperatures differed by almost $19 \%$. The C-B Method's lower evaporating temperature should produce a higher capacity than the ARI Test evaporating temperature, but this was not the case. The lower evaporating temperature produced a $12.3 \%$ lower capacity which was combined with a $14.3 \%$ lower CD Unit power to produce an almost equivalent $\operatorname{EER}(82)$. 
Table 5.2.1: Mixed system B-Test comparison of ARI tested and C-B Method results

\begin{tabular}{|c|c|c|c|c|c|c|c|c|c|c|c|c|c|c|c|}
\hline \multirow[t]{2}{*}{ Coil } & \multicolumn{3}{|c|}{$\begin{array}{l}T_{\text {evap }} \\
{ }^{\circ} \mathrm{C}\left({ }^{\circ} \mathrm{F}\right)\end{array}$} & \multicolumn{3}{|c|}{$\begin{array}{c}\text { CD Unit Power } \\
\text { W }\end{array}$} & \multicolumn{3}{|c|}{$\begin{array}{c}\text { Fan Power } \\
\text { W }\end{array}$} & \multicolumn{3}{|c|}{$\begin{array}{c}Q(82)^{c} \\
W(B t u / h)\end{array}$} & \multicolumn{3}{|c|}{$\begin{array}{c}\text { EER(82) } \\
\text { W/W (Btu/W h) }\end{array}$} \\
\hline & $\begin{array}{c}\text { C-B } \\
\text { Method }\end{array}$ & $\begin{array}{l}\text { ARI } \\
\text { Test }\end{array}$ & $\begin{array}{c}\text { Difference } \\
\text { (C-B } \\
\text { Method - } \\
\text { ARI) } \\
\end{array}$ & $\begin{array}{c}\text { C-B } \\
\text { Method }\end{array}$ & $\begin{array}{l}\text { ARI } \\
\text { Test }\end{array}$ & $\begin{array}{c}\text { Percent } \\
\text { Diff } \\
\% \\
\end{array}$ & $\begin{array}{c}\text { C-B } \\
\text { Method }\end{array}$ & $\begin{array}{l}\text { ARI } \\
\text { Test }\end{array}$ & $\begin{array}{l}\text { Percent } \\
\text { Diff } \\
\%\end{array}$ & $\begin{array}{c}\text { C-B } \\
\text { Method }\end{array}$ & $\begin{array}{l}\text { ARI } \\
\text { Test }\end{array}$ & $\begin{array}{l}\text { Percent } \\
\text { Diff } \\
\%\end{array}$ & $\begin{array}{c}\text { C-B } \\
\text { Method }\end{array}$ & ARI Test & $\begin{array}{l}\text { Percent } \\
\text { Diff } \\
\%\end{array}$ \\
\hline 1 & $\begin{array}{c}7.94 \\
(46.3)\end{array}$ & $\begin{array}{c}7.94 \\
(46.3)\end{array}$ & $0.0(0.0)$ & 2530 & 2146 & 17.9 & 365 & 368 & -0.7 & $\begin{array}{c}9763 \\
(33313)\end{array}$ & $\begin{array}{c}10017 \\
(34178) \\
\end{array}$ & -2.5 & $\begin{array}{c}3.37 \\
(11.51)\end{array}$ & $\begin{array}{c}3.99 \\
(13.60)\end{array}$ & -15.4 \\
\hline 2 & $\begin{array}{c}5.0 \\
(41.0)\end{array}$ & $\begin{array}{c}6.50 \\
(43.7) \\
\end{array}$ & $-1.5(-2.7)$ & 1953 & 2090 & -6.6 & 294 & 294 & -0.1 & $\begin{array}{c}6895 \\
(23525) \\
\end{array}$ & $\begin{array}{c}6559 \\
(22381) \\
\end{array}$ & 5.1 & $\begin{array}{c}3.07 \\
(10.47) \\
\end{array}$ & $\begin{array}{c}2.75 \\
(9.39) \\
\end{array}$ & 11.5 \\
\hline 3 & $\begin{array}{c}7.72 \\
(45.9)\end{array}$ & $\begin{array}{c}6.83 \\
(44.3)\end{array}$ & $0.9(1.6)$ & 2528 & 2255 & 12.1 & 348 & 334 & 4.1 & $\begin{array}{c}9717 \\
(33155)\end{array}$ & $\begin{array}{c}10164 \\
(34680)\end{array}$ & -4.4 & $\begin{array}{c}3.38 \\
(11.53)\end{array}$ & $\begin{array}{c}3.92 \\
(13.39)\end{array}$ & -13.9 \\
\hline $4^{d}$ & $\begin{array}{c}8.89 \\
(48.0)\end{array}$ & $\begin{array}{c}9.72 \\
(49.5) \\
\end{array}$ & $-0.8(-1.5)$ & 1471 & 1670 & -11.9 & 288 & 232 & 24.2 & $\begin{array}{c}5410 \\
(18458)\end{array}$ & $\begin{array}{c}5338 \\
(18215) \\
\end{array}$ & 1.3 & $\begin{array}{c}3.07 \\
(10.49) \\
\end{array}$ & $\begin{array}{c}2.81 \\
(9.58) \\
\end{array}$ & 9.5 \\
\hline 5 & $\begin{array}{c}8.50 \\
(47.3)\end{array}$ & $\begin{array}{c}9.67 \\
(49.4)\end{array}$ & $-1.2(-2.1)$ & 2024 & 1840 & 10.0 & 271 & 310 & -12.6 & $\begin{array}{c}7402 \\
(25258) \\
\end{array}$ & $\begin{array}{c}7207 \\
(24591) \\
\end{array}$ & 2.7 & $\begin{array}{c}3.23 \\
(11.01) \\
\end{array}$ & $\begin{array}{c}3.35 \\
(11.44) \\
\end{array}$ & -3.8 \\
\hline 6 & $\begin{array}{c}5.39 \\
(41.7)\end{array}$ & $\begin{array}{c}6.50 \\
(43.7)\end{array}$ & $-1.1(-2.0)$ & 4257 & 4340 & -1.9 & $784^{a}$ & $848^{\mathrm{b}}$ & -7.5 & $\begin{array}{c}16593 \\
(56616)\end{array}$ & $\begin{array}{c}15613 \\
(53274)\end{array}$ & 6.3 & $\begin{array}{c}3.29 \\
(11.23) \\
\end{array}$ & $\begin{array}{c}3.01 \\
(10.27)\end{array}$ & 9.4 \\
\hline 7 & $\begin{array}{c}2.50 \\
(36.5)\end{array}$ & $\begin{array}{c}7.22 \\
(45.0)\end{array}$ & $-4.7(-8.5)$ & 2897 & 3380 & -14.3 & $440^{a}$ & $439^{b}$ & 0.1 & $\begin{array}{c}9236 \\
(31513) \\
\end{array}$ & $\begin{array}{c}10529 \\
(35926) \\
\end{array}$ & -12.3 & $\begin{array}{c}2.77 \\
(9.44) \\
\end{array}$ & $\begin{array}{c}2.76 \\
(9.41) \\
\end{array}$ & 0.4 \\
\hline 8 & $\begin{array}{c}5.06 \\
(41.1)\end{array}$ & $\begin{array}{c}6.39 \\
(43.5)\end{array}$ & $-1.3(-2.4)$ & 3196 & 4143 & -22.9 & 507 & 507 & -0.1 & $\begin{array}{c}11500 \\
(39238)\end{array}$ & $\begin{array}{c}12508 \\
(42680)\end{array}$ & -8.1 & $\begin{array}{c}3.11 \\
(10.60)\end{array}$ & $\begin{array}{c}2.69 \\
(9.18)\end{array}$ & 15.4 \\
\hline 9 & $\begin{array}{c}5.39 \\
(41.7) \\
\end{array}$ & $\begin{array}{c}5.89 \\
(42.6) \\
\end{array}$ & $-0.5(-0.9)$ & 1943 & 1954 & -0.6 & $364^{a}$ & $382^{b}$ & -4.7 & $\begin{array}{c}7253 \\
(24749) \\
\end{array}$ & $\begin{array}{c}6972 \\
(23790) \\
\end{array}$ & 4.0 & $\begin{array}{c}3.14 \\
(10.73) \\
\end{array}$ & $\begin{array}{c}2.98 \\
(10.18) \\
\end{array}$ & 5.4 \\
\hline
\end{tabular}

a) Fan power measured by NIST. b) Fan power measured by ARI contracted testing facility. c) Q(82) included the fan heat correction for coils with no fan.

d) NIST airflow was $262 \mathrm{~m}^{3} / \mathrm{h}$ (154 scfm) higher than the ARI tested airflow for Coil 4. 


\section{3: SEER comparison}

Table 5.3.1 presents SEER values from the ARI database and the C-B Method for the $99^{\text {th }}$ percentile cyclic degradation coefficients. With the exception of Coil 6 , where the predicted SEER exceeds the measured SEER by $0.6 \%$, the SEER values obtained from the C-B Method are lower than the measured values by as much as $27 \%$. Four C-B Method SEERs agreed to within $\pm 5 \%$ of the measured SEERs. Figure 5.3 .1 shows that this level of agreement can be attributed to offsetting errors between EERs and SEER/EER Multipliers, (1-0.5 $\left.C_{D}\right)$, especially considering the large differences in cyclic degradation coefficient between the NIST $99^{\text {th }}$ percentile and ARI database. These large differences in degradation coefficient indicate that more information is needed to accurately determine a representative $C_{D}$ value. Clearly, the use of a fixed value for the degradation coefficient cannot reliably reproduce $A R I$ database values.

Table 5.3.1: SEERs from ARI value and C-B Method

\begin{tabular}{|c|c|c|c|c|c|c|}
\hline Coil & $\begin{array}{c}\text { SEER } \\
\text { from ARI } \\
\text { values } \\
\text { Btu/(W/h) }\end{array}$ & $\begin{array}{c}\text { SEER } \\
\text { from C-B } \\
\text { Method } \\
\text { using 99 } \\
\text { percentile } C_{d}\end{array}$ & $\begin{array}{c}\text { SEER }{ }^{(1)} \\
\text { Diff } \\
\%\end{array}$ & $\begin{array}{c}\text { ARI } \\
\text { SEER/EER } \\
\text { Multiplier } \\
\left(1-0.5 C_{d}\right)\end{array}$ & $\begin{array}{c}\text { NIST 99 } \\
\text { Percentile } \\
\text { SEER/EER } \\
\text { Multiplier } \\
\left(1-0.5 C_{d}\right)\end{array}$ & $\begin{array}{c}\left.\text { (ARI - NIST 99 }^{\text {th }}\right) \\
\text { SEER/EER } \\
\text { Multiplier }\end{array}$ \\
\hline 1 & 13.87 & 10.13 & -27.0 & 0.931 & 0.880 & 0.051 \\
\hline 2 & 9.63 & 9.22 & -4.3 & 0.983 & 0.880 & 0.103 \\
\hline 3 & 13.33 & 10.14 & -23.9 & 0.964 & 0.880 & 0.084 \\
\hline $4^{*}$ & 9.79 & 9.23 & -5.7 & 0.980 & 0.880 & 0.100 \\
\hline 5 & 10.01 & 9.68 & -3.3 & 0.876 & 0.880 & -0.004 \\
\hline 6 & 9.82 & 9.88 & 0.6 & 0.976 & 0.880 & 0.096 \\
\hline 7 & 9.56 & 8.31 & -13.1 & 0.977 & 0.880 & 0.097 \\
\hline 8 & 10.17 & 9.32 & -8.4 & 0.949 & 0.880 & 0.069 \\
\hline 9 & 9.78 & 9.44 & -3.5 & 0.961 & 0.880 & 0.081 \\
\hline
\end{tabular}

(1) SEER Diff $=100 \%$ (NIST - ARI)/ARI

${ }^{(2)}$ ARI SEER/EER multiplier was calculated from SEER $=\left(1-0.5 C_{d}\right)$ EER(82)

${ }^{*}$ Coil 4 airflow was too high and results cannot be compared directly to ARI tests.

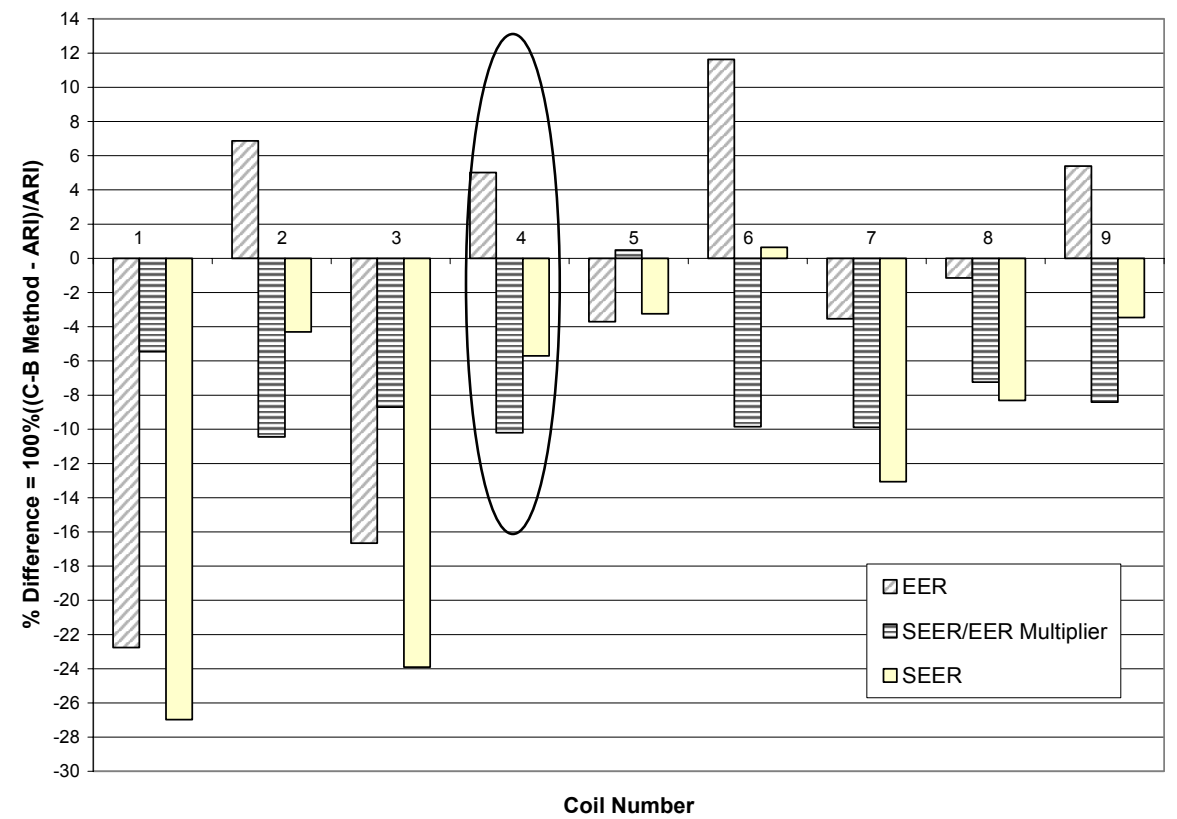

Figure 5.3.1: ARI Values and C-B Method SEER/EER Multiplier, EER, and SEER 


\section{6: GENERATING AN EVAPORATOR CAPACITY LINE USING EVAP-COND}

In this study, we developed evaporator capacity lines based on measured capacities at several different evaporator exit saturation temperatures with constant superheat and inlet quality. Controlling these three parameters makes coil tests more time consuming than complete system tests. For this reason, we explored the possibility to minimize the laboratory effort by using an evaporator simulation model. A simulation model can be tuned to predict the measured capacity, which can then be used to provide capacities at other saturation temperatures needed to generate the evaporator capacity line. To demonstrate this approach, we used the EVAP-COND finned-tube heat exchanger simulation package (NIST 2003).

EVAP-COND uses coil design parameters (including refrigerant circuitry) and refrigerant and air parameters as input to calculate the capacity of the heat exchanger. The model allows the user to tune its prediction to experimental data by adjusting the correction factors for the air-side heat transfer coefficient, refrigerant-side heat transfer coefficient, and refrigerantside pressure drop. In our case, we considered Coil 9 and selected appropriate values for these factors, shown in Table 6.1, so the model predictions agreed with the test results at the $7.4^{\circ} \mathrm{C}\left(45.4^{\circ} \mathrm{F}\right)$ saturation temperature. Simulations at additional saturation temperatures allowed generation of a linear capacity fit for the coil.

Table 6.1: EVAP-COND correction parameters for Coil 9

\begin{tabular}{|c|c|c|c|c|c|c|}
\hline $\begin{array}{c}T_{\text {evap }}{ }^{\circ} \mathrm{C} \\
\left({ }^{\circ} \mathrm{F}\right)\end{array}$ & $\mathrm{R}$ & $\Delta \mathrm{P}$ & $\mathrm{A}$ & $\begin{array}{c}q \\
\text { Predicted } \\
\mathrm{W}(\mathrm{Btu} / \mathrm{h})\end{array}$ & $\begin{array}{c}q \\
\text { Measured } \\
\mathrm{W}(\mathrm{Btu} / \mathrm{h})\end{array}$ & $\begin{array}{c}q_{\text {diff }} \\
\%\end{array}$ \\
\hline $\begin{array}{c}7.4 \\
(45.4)\end{array}$ & 1.15 & 2.00 & 1.30 & $\begin{array}{c}6249 \\
(21323)\end{array}$ & $\begin{array}{c}6242 \\
(21300)\end{array}$ & 0.1 \\
\hline
\end{tabular}

Figure 6.1 shows cooling capacities for Coil 9 from the laboratory measurements, the EVAPCOND simulations, and the corresponding capacity lines as a function of evaporating temperature. The capacity lines almost overlap. Consequently, the C-B Method performance predictions for mixed system using Coil 9 were within $0.5 \%$ for the two capacity lines, as shown in Figure 6.2.

It is possible that the approach presented here could be extended to other evaporators that use the same air-side and refrigerant-side heat transfer surfaces; i.e., once EVAP-COND adjustable factors for air-side heat transfer, refrigerant-side heat transfer, and refrigerant pressure drop are determined for one coil, they could be applied to other coils using the same surfaces. 


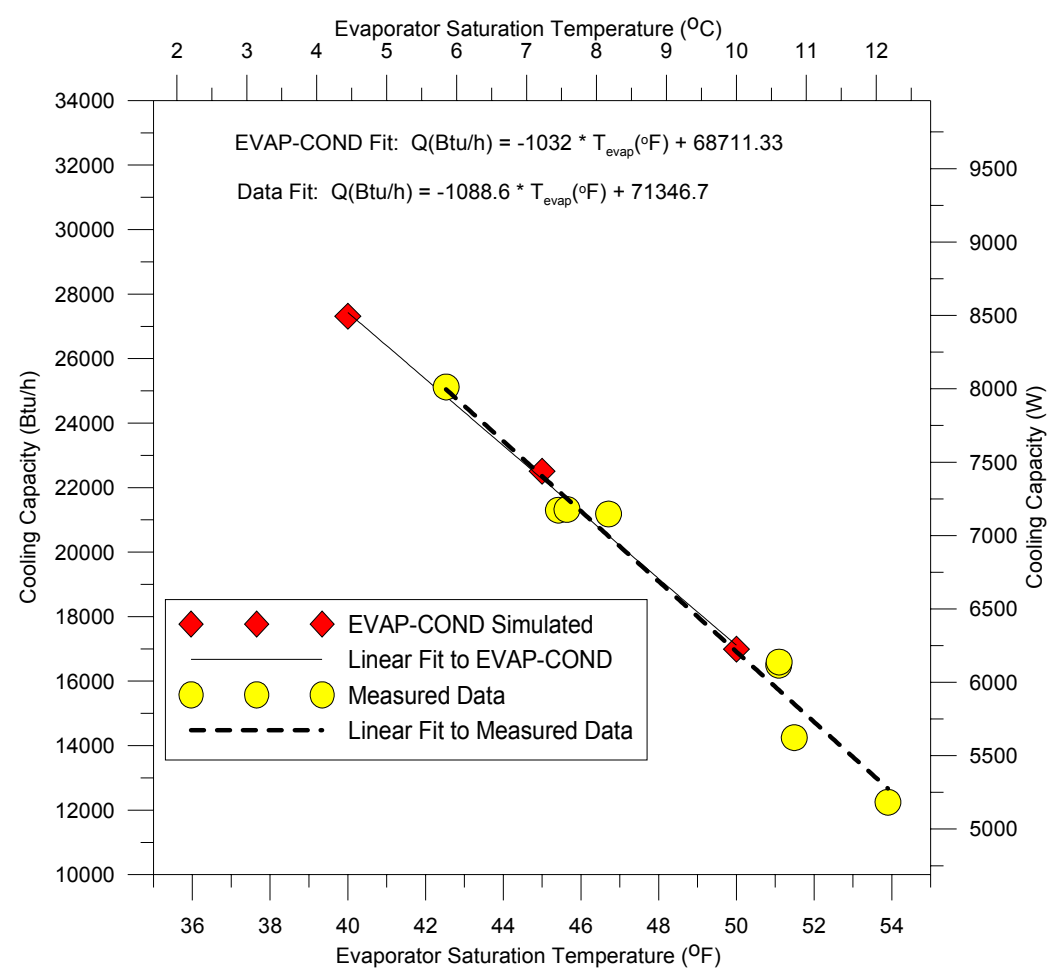

Figure 6.1: Coil 9 simulated and measured cooling capacity

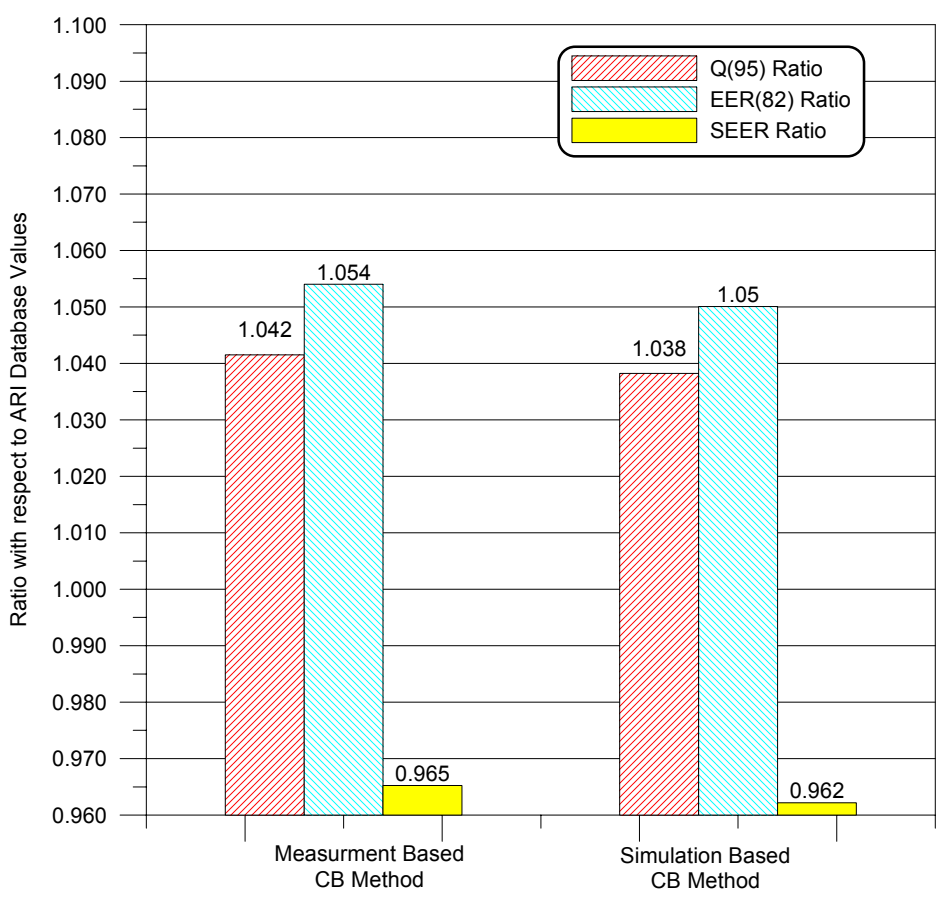

Figure 6.2: C-B Method Q(95), EER(82) and SEER ratio with respect to the ARI database using the measured and simulated Coil 9 capacity 


\section{7: UNCERTAINTY OF THE C-B METHOD}

\section{1: Uncertainty of the evaporator capacity linear fit}

We used Coil 9 data as an example to evaluate the uncertainty of the evaporator capacity linear fit. This is the uncertainty that would result if someone used this linear fit to calculate capacity at a known evaporator temperature. The data set used to generate the linear fit consisted of 8 tests. A linear equation has two adjustable parameters; therefore, the fit had 6 degrees of freedom (DOF $=n-2$ ). Table 7.1.1 summarizes the linear fit parameters (slope and intercept) and related fit statistics.

Table 7.1.1: Coil 9 evaporator linear fit equation statistics

\begin{tabular}{|c|c|c|c|c|c|}
\hline $\begin{array}{c}\text { Correlation } \\
\text { Coefficient } \\
\mathrm{R}^{2}\end{array}$ & $\begin{array}{c}\text { DOF } \\
\text { Adjusted } \mathrm{R}^{2}\end{array}$ & $\begin{array}{c}\text { Fit Standard } \\
\text { Error, W (Btu/h) }\end{array}$ & F-Value & \multirow{2}{*}{\multicolumn{2}{|c|}{$\begin{array}{c}n=8 \text { data points } \\
\text { DOF }=n-2=6\end{array}$}} \\
\hline 0.972835 & 0.961969 & $\begin{array}{c}226.28 \\
(772.093) \\
\end{array}$ & 214.872 & & \\
\hline \multirow[t]{2}{*}{ Parameter } & \multirow[t]{2}{*}{ Value } & \multirow[t]{2}{*}{ Standard Error } & \multirow[t]{2}{*}{ t-value } & \multirow{2}{*}{\multicolumn{2}{|c|}{$\begin{array}{c}95 \% \text { Confidence Limits on the } \\
\text { Mean Value } \\
\text { Minimum Maximum }\end{array}$}} \\
\hline & & & & & \\
\hline $\begin{array}{c}\text { B (intercept), W } \\
(\text { Btu/h) }\end{array}$ & $\begin{array}{l}20909.6 \\
(71346.5)\end{array}$ & $\begin{array}{c}1058.35 \\
(3611.22)\end{array}$ & 19.757 & $\begin{array}{c}18309.1 \\
(62473.4)\end{array}$ & $\begin{array}{c}23510.1 \\
(80219.6)\end{array}$ \\
\hline $\begin{array}{l}\text { A (slope), W/ } /{ }^{\circ} \mathrm{C} \\
\left.\text { (Btu/h }{ }^{\circ} \mathrm{F}\right)\end{array}$ & $\begin{array}{c}-574.32 \\
(-1088.7)\end{array}$ & $\begin{array}{c}39.18 \\
(74.27)\end{array}$ & -14.659 & $\begin{array}{l}-670.56 \\
(-1271.2)\end{array}$ & $\begin{array}{l}-478.0 \\
(-906.2)\end{array}$ \\
\hline $\mathrm{S}_{\mathrm{xx}}$ & 108.0774 & & & & \\
\hline Mean $T_{\text {evap }}$ & $\begin{array}{c}9.2 \\
(48.5)\end{array}$ & Mean $q$ & $\begin{array}{c}5440 \\
(18562.8)\end{array}$ & & \\
\hline
\end{tabular}

At a $95 \%$ confidence level, the linear intercept is equal to $20909.6 \pm 2600.45 \mathrm{~W}(71346.5 \pm$ 8873.1 Btu/h), and the linear slope is equal to $-574.32 \pm 39.18 \mathrm{~W} /{ }^{\circ} \mathrm{C}(-1088.7 \pm$ $\left.182.5 \mathrm{Btu} / \mathrm{h}{ }^{\circ} \mathrm{F}\right)$. The confidence bands are determined by subtracting the minimum $95 \%$ limit from the maximum $95 \%$ limit and dividing by two (or taking the fit standard error and multiplying by the appropriate t-value). With confidence limits as a percentage value, the linear intercept is $20909.6 \mathrm{~W}(71346.5 \mathrm{Btu} / \mathrm{h}) \pm 12.4 \%$, and the linear slope is $-574.32 \mathrm{~W} /{ }^{\circ} \mathrm{C}\left(-1088.7 \mathrm{Btu} / \mathrm{h}^{\circ} \mathrm{F}\right) \pm 16.8 \%$.

For capacity predictions using the linear correlation, the confidence interval for the mean value at a particular point is given by (Ott 1984):

$$
\pm t \cdot \hat{\sigma} \sqrt{\frac{1}{n}+\frac{(x-\bar{x})^{2}}{\mathrm{~S}_{\mathrm{xx}}}}
$$

where $\hat{\sigma}=\sqrt{\frac{\mathrm{SSE}}{n-2}}=$ fit standard error

$S_{x x}=\sum\left(x^{2}\right)-\frac{\left(\sum x\right)^{2}}{n}$

$t$ - two tailed t-value for the appropriate confidence level with DOF $=n-2$

$\hat{\sigma}$ - estimated standard deviation equal to the fit standard error 
$\bar{x}$ - mean value of the $\mathrm{x}$-variables or, in this case, the mean value of the evaporating temperatures used to generate the linear fit

$x$ - independent variable or, in this case, the evaporator temperature

At a $95 \%$ confidence level, the t-value for six DOF is 2.447 from a table of the percentage points of the t-distribution (Ott 1984). From Table 7.1.1, the fit standard error was $226.28 \mathrm{~W}$ $(772.093 \mathrm{Btu} / \mathrm{h})$, and $\mathrm{S}_{\mathrm{xx}}$ was $33.36^{\circ} \mathrm{C}^{2}\left(108.08^{\circ} \mathrm{F}^{2}\right)$. Substituting these values into Equation 7.1 .1 yields:

$$
\pm 2.447 \cdot 772.093 \sqrt{0.125+\frac{(x-48.485)^{2}}{108.08}}
$$

Knowing the confidence interval for a given confidence level and the predicted value, we can calculate the upper and lower confidence bands for the mean value of the cooling capacity sampled multiple times at a particular value of the evaporator saturation temperature within the range of the evaporator saturation temperature data. Figure 7.1.1 plots the evaporator capacity line, $90 \%$ and $95 \%$ confidence bands, and $5 \%$ offset lines for Coil 9. The figure shows that the $90 \%$ and $95 \%$ confidence lines are very close to each other; they are within the $\pm 5 \%$ offset lines for the majority of the saturation temperature range except the lowest and highest saturation temperatures due to the smaller number of data points associated with the end points of the temperature range.

Once the rater has used the C-B Method (Equation 4.1.2) to determine the evaporating temperature for the condensing unit and the mixed evaporator, the analysis of section 7.1 provides the uncertainty in the mean value of the cooling capacity sampled at that particular evaporator temperature. For Coil 9 and its CD Unit, the evaporating temperature was $6.2^{\circ} \mathrm{C}$ $\left(43.2^{\circ} \mathrm{F}\right)$ which produced a cooling capacity, $q(95)$, of $7114 \mathrm{~W}(24273 \mathrm{Btu} / \mathrm{h})$ with an uncertainty of $\pm 341 \mathrm{~W}(1164 \mathrm{Btu} / \mathrm{h})$ or $\pm 4.8 \%$ on the mean value at a $95 \%$ confidence level. Subtracting the fan heat, which has $a \pm 3 \%$ uncertainty, yields the numbers seen in Table 5.1 .1 or a rated Q(95) of $6749 \mathrm{~W}(23030 \mathrm{Btu} / \mathrm{h}) \pm 341 \mathrm{~W}(1165 \mathrm{Btu} / \mathrm{h})( \pm 5.1 \%)$ at a $95 \%$ confidence level. Table 7.1.2 summarizes the uncertainty results for the remaining evaporators. 


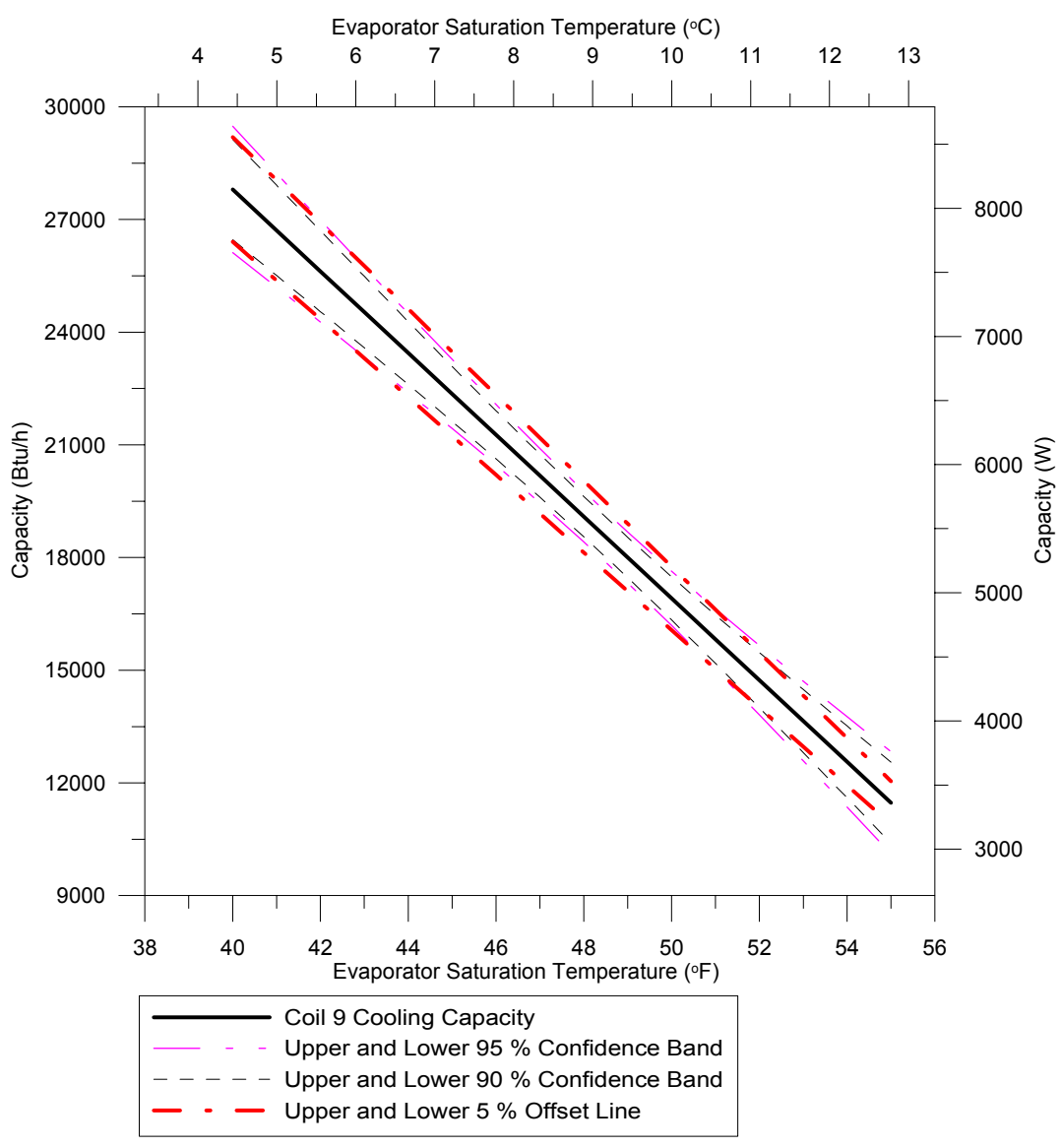

Figure 7.1.1: Coil 9 evaporator cooling capacity, confidence and error bands on the mean predicted value of the cooling capacity sampled at a particular evaporator saturation temperature

Table 7.1.2: Evaporator Q(95) linear fit uncertainty

\begin{tabular}{|c|c|c|c|c|}
\hline Coil & $\begin{array}{c}T_{\text {evap }} \\
{ }^{\circ} \mathrm{C}\left({ }^{\circ} \mathrm{F}\right)\end{array}$ & $\begin{array}{c}\text { Q(95) } \\
\mathrm{W}(\text { Btu/h) }\end{array}$ & $\begin{array}{c}95 \% \text { Confidence Level on } \\
\text { the Mean, } \\
\text { Uncertainty Q(95) }\end{array}$ & \% Uncertainty \\
\hline 1 & $8.11(46.60)$ & $9624(32839)$ & $541(1847)$ & 5.6 \\
\hline 2 & $5.84(42.51)$ & $6344(21647)$ & $334(1138)$ & 5.3 \\
\hline 3 & $7.93(46.27)$ & $9585(32705)$ & $267(910)$ & 2.8 \\
\hline $4^{*}$ & $9.94(49.89)$ & $4894(16700)$ & $127(434)$ & 2.6 \\
\hline 5 & $9.43(48.98)$ & $6917(23601)$ & $192(655)$ & 6.0 \\
\hline 6 & $6.2(43.19)$ & $15756(53761)$ & $939(3205)$ & 1.5 \\
\hline 7 & $4.5(40.17)$ & $8340(28456)$ & $124(424)$ & 2.4 \\
\hline 8 & $5.9(42.6)$ & $10878(37115)$ & $260(886)$ & 5.1 \\
\hline 9 & $6.2(43.24)$ & $6749(23030)$ & $342(1166)$ & \\
\hline
\end{tabular}

${ }^{*}$ Coil 4 airflow was too high and results cannot be compared directly to ARI tests. 


\section{2: Uncertainty of C-B Method Q(95) and EER}

The ARI database, $q(95), q(82)$ and $p(82)$ linear fits of Table 4.1.1, NIST capacity linear fits of Table 3.4.1, Equation 4.1.2, and fan heat were used to calculate the uncertainty in the CB Method Q(95) and EER.

$$
\begin{gathered}
q_{C D}=A_{C D} T_{\text {evap }}+B_{C D}=q_{\text {mixed }}=A_{\text {mixed }} T_{\text {evap }}+B_{\text {mixed }} \\
T_{\text {evap }}=\frac{B_{\text {mixed }}-B_{C D}}{A_{C D}-A_{\text {mixed }}} \\
p_{C D}=b_{C D}+a_{C D} T_{\text {evap }}
\end{gathered}
$$

Substituting Equation 4.1.2 into Equation 4.1.1 and 4.1.4 yields an expression for the cooling capacity and CD Unit power of the mixed system.

$$
\begin{gathered}
q_{C D}=q_{\text {mixed }}=A_{C D}\left(\frac{B_{\text {mixed }}-B_{C D}}{A_{C D}-A_{\text {mixed }}}\right)+B_{C D} \\
p_{C D}=b_{C D}+a_{C D}\left(\frac{B_{\text {mixed }}-B_{C D}}{A_{C D}-A_{\text {mixed }}}\right)
\end{gathered}
$$

The fit standard errors for the CD Unit capacity and power linear coefficients are unknown. If we assume the fit standard error for the CD Unit capacity is similar (the same percentage) to that obtained for the evaporator tests, power coefficient uncertainties are $3 \%$, power and capacity fits have no covariance, and the covariance of the slope and intercept are equal for the CD Unit and evaporator tests, then we may calculate an uncertainty for the mixed system capacity and power.

In general the capacity is a function of the four linear fit coefficients and the power is a function of six linear fit coefficients:

$$
\begin{gathered}
q_{C D}=q_{\text {mixed }}=f\left(A_{C D}, B_{C D}, A_{\text {mixed }}, B_{\text {mixed }}\right) \\
q_{C D}=q_{\text {mixed }}=f\left(a_{C D}, b_{C D}, A_{C D}, B_{C D}, A_{\text {mixed }}, B_{\text {mixed }}\right)
\end{gathered}
$$

Since the slope and intercept are not independent in Equations 7.2.1 and 7.2.2, we must include covariance in the form of a correlation coefficient (Coleman and Steele 1989). In general terms this becomes:

$$
\begin{gathered}
U_{q}{ }^{2}=\left(\frac{\partial q}{\partial A_{C D}} U_{A_{C D}}\right)^{2}+\left(\frac{\partial q}{\partial B_{C D}} U_{B_{C D}}\right)^{2}+\left(\frac{\partial q}{\partial A_{\text {mixed }}} U_{A_{\text {mixed }}}\right)^{2}+\left(\frac{\partial q}{\partial B_{\text {mixed }}} U_{B_{\text {mixed }}}\right)^{2} \\
+2\left(\frac{\partial q}{\partial A_{C D}}\right)\left(\frac{\partial q}{\partial B_{C D}}\right) \rho_{A_{C D} B_{C D}} U_{A_{C D}} U_{B_{C D}}+2\left(\frac{\partial q}{\partial A_{\text {mixed }}}\right)\left(\frac{\partial q}{\partial B_{\text {mixed }}}\right) \rho_{A_{\text {mixed }} B_{\text {mixed }}} U_{A_{\text {mixed }}} U_{B_{\text {mixed }}}
\end{gathered}
$$

Here the partial derivatives are taken from Equation 7.2.1 with respect to the various coefficients. A similar procedure is necessary for the CD Unit power equation with the addition of the two extra terms for the power equation linear fit coefficients. The fan power and resulting heat were assumed to have an uncertainty of $\pm 3 \%$. The fan heat must be 
subtracted from the cooling capacity, and the fan power must be added to the CD Unit power with their variances added to produce the cooling capacity and total power final values and associated uncertainties.

EER is the ratio of $Q(82)$ and $P(82)$.

$$
E E R=\frac{Q(82)}{P(82)}
$$

The propagation of the capacity uncertainty and the power uncertainty through Equation 1.1 produces a resulting uncertainty in the EER. We assumed no covariance between capacity and power. The resulting uncertainty in EER is shown below in Table 7.2.1.

Table 7.2.1: C-B Method $Q(95), Q(82), P(82)$, and EER uncertainty at the $95 \%$ confidence level on the mean value

\begin{tabular}{|c|c|c|c|c|c|c|c|c|c|c|}
\hline Coil & $T_{\text {evap }}(82)$ & $T_{\text {evap }}(95)$ & $\begin{array}{c}Q(95) \\
W \\
(\text { Btu/h) }\end{array}$ & $\begin{array}{c}* \% \\
U_{Q(95)}^{*}\end{array}$ & $\begin{array}{c}Q(82) \\
W \\
(B t u / h)\end{array}$ & $\begin{array}{c}{ }^{\mathrm{a}} \% \\
\mathrm{U}_{\mathrm{Q}(82)}\end{array}$ & $\begin{array}{c}P(82) \\
W\end{array}$ & $U_{P(82)}^{\%}$ & $\begin{array}{c}\text { EER } \\
\text { W/W } \\
\text { (Btu/W h) }\end{array}$ & $\stackrel{\%}{\%}$ \\
\hline 1 & $\begin{array}{c}7.9 \\
(46.3)\end{array}$ & $\begin{array}{c}8.1 \\
(46.6)\end{array}$ & $\begin{array}{c}9624 \\
(32839)\end{array}$ & 15.3 & $\begin{array}{c}9763 \\
(33313)\end{array}$ & 15.7 & 2895 & 3.7 & $\begin{array}{c}3.37 \\
(11.51)\end{array}$ & 16.1 \\
\hline 2 & $\begin{array}{c}5.0 \\
(41.0)\end{array}$ & $\begin{array}{c}5.8 \\
(42.5)\end{array}$ & $\begin{array}{c}6344 \\
(21647)\end{array}$ & 4.4 & $\begin{array}{c}6895 \\
(23525)\end{array}$ & 4.5 & 2246 & 9.7 & $\begin{array}{c}3.07 \\
(10.47)\end{array}$ & 10.7 \\
\hline 3 & $\begin{array}{c}7.7 \\
(45.9) \\
\end{array}$ & $\begin{array}{c}7.9 \\
(46.3) \\
\end{array}$ & $\begin{array}{c}9585 \\
(32705) \\
\end{array}$ & 8.3 & $\begin{array}{c}9717 \\
(33155) \\
\end{array}$ & 8.5 & 2876 & 2.8 & $\begin{array}{c}3.38 \\
(11.53) \\
\end{array}$ & 9.0 \\
\hline $4^{*}$ & $\begin{array}{c}8.9 \\
(48.0) \\
\end{array}$ & $\begin{array}{c}9.9 \\
(49.9)\end{array}$ & $\begin{array}{c}4894 \\
(16700)\end{array}$ & 7.5 & $\begin{array}{c}5410 \\
(18458)\end{array}$ & 6.2 & 1760 & 3.4 & $\begin{array}{c}3.07 \\
(10.49)\end{array}$ & 7.1 \\
\hline 5 & $\begin{array}{c}8.5 \\
(47.3)\end{array}$ & $\begin{array}{c}9.4 \\
(49.0)\end{array}$ & $\begin{array}{c}6917 \\
(23601)\end{array}$ & 8.2 & $\begin{array}{c}7402 \\
(25258)\end{array}$ & 7.0 & 2295 & 5.8 & $\begin{array}{c}3.23 \\
(11.01)\end{array}$ & 9.1 \\
\hline 6 & $\begin{array}{c}5.4 \\
(41.7) \\
\end{array}$ & $\begin{array}{c}6.2 \\
(43.2)\end{array}$ & $\begin{array}{c}15756 \\
(53761)\end{array}$ & 9.0 & $\begin{array}{c}16593 \\
(56616)\end{array}$ & 8.1 & 5041 & 4.1 & $\begin{array}{c}3.29 \\
(11.23)\end{array}$ & 9.1 \\
\hline 7 & $\begin{array}{c}2.5 \\
(36.5) \\
\end{array}$ & $\begin{array}{c}4.5 \\
(40.2) \\
\end{array}$ & $\begin{array}{c}8340 \\
(28456) \\
\end{array}$ & 6.3 & $\begin{array}{c}9236 \\
(31513) \\
\end{array}$ & 4.6 & 3337 & 2.5 & $\begin{array}{c}2.77 \\
(9.44) \\
\end{array}$ & 5.2 \\
\hline 8 & $\begin{array}{c}5.1 \\
(41.1)\end{array}$ & $\begin{array}{c}5.9 \\
(42.6)\end{array}$ & $\begin{array}{c}10877 \\
(37115)\end{array}$ & 5.2 & $\begin{array}{c}11500 \\
(39238)\end{array}$ & 5.6 & 3703 & 16.9 & $\begin{array}{c}3.11 \\
(10.60)\end{array}$ & 17.8 \\
\hline 9 & $\begin{array}{c}5.4 \\
(41.7) \\
\end{array}$ & $\begin{array}{c}6.2 \\
(43.2) \\
\end{array}$ & $\begin{array}{c}6749 \\
(23030) \\
\end{array}$ & 10.8 & $\begin{array}{c}7253 \\
(24749) \\
\end{array}$ & 9.3 & 2307 & 3.3 & $\begin{array}{c}3.14 \\
(10.73) \\
\end{array}$ & 9.9 \\
\hline
\end{tabular}

a U: uncertainty

${ }^{*}$ Coil 4 airflow was too high and results cannot be compared directly to ARI tests.

The uncertainty values may be skewed higher by the assumption of equal percentage uncertainties for the evaporator and CD Unit linear fit capacity coefficients. It is likely that the manufacturers of the CD Units have much larger data sets than those collected in this work; therefore, the uncertainty percentages may be lower than those presented in Table 7.2.1.

\section{8: CONCLUDING REMARKS}

This report examines the application of the C-B Method to determine $Q(95)$ and SEER of mixed air conditioners was studied on a sample of eight mixed systems. An independent certification laboratory performed mixed system tests and shipped the mixed evaporators to NIST for testing in NIST's environmental chambers. We implemented the C-B Method for 
Q(95) and SEER ratings using condensing unit performance correlations obtained from ARI, the evaporator capacity correlations developed at NIST, and the $99^{\text {th }}$ percentile value of cyclic degradation coefficients identified for the equipment studied. We compared the obtained $Q(95)$ and SEER ratings to the test-obtained values from the certification laboratory (referred to as ARI values).

The C-B Method produced Q(95) results that were within $\pm 5 \%$ of the ARI tested values for five of eight coils. Among the remaining cases, one $Q(95)$ was overpredicted by $9.4 \%$ and two $Q(95)$ were underpredicted by $13.6 \%$ and $17.6 \%$. In two of the five cases with $\pm 5 \%$ agreement, the good Q(95) predictions were obtained as a result of error offsetting between evaporator and condensing unit performance correlations with respect to the system tested capacities, as evidenced by the misprediction of the evaporator saturation temperature by the C-B Method in these two cases.

The $Q(82)$ values from the C-B method were within $\pm 5 \%$ of the ARI tested values for four of eight coils with six of eight coils being within $\pm 7 \%$. Of all the factors contributing to the differences in EER(82), the CD Unit power had the largest effect. Using the CD Unit linear fits for power, the C-B Method predicted EER(82) to within $\pm 5 \%$ for only two coils. Using the ARI Test values for CD Unit power, the C-B Method predicted EER(82) to within $\pm 5 \%$ for four of eight coils and within $\pm 8 \%$ for six of eight coils. Clearly good representations of CD Unit power must be attained to produce consistently correct values of EER(82).

Regarding SEER values, four of eight predictions were within $\pm 5 \%$ of the ARI tested values, but offsetting of errors played a role in this agreement due to conservative $\left(99^{\text {th }}\right.$ percentile) selection of the cyclic degradation coefficient used by the C-B Method. For the same reason seven of eight SEER predictions were below the test-derived SEERs.

The uncertainty analysis of the C-B Method showed that the $95 \%$ confidence level on the mean predictions averaged $3.8 \%$ for A-Test capacity and $10.4 \%$ for EER. The analysis also showed the importance of careful collection of data; when the evaporator capacity linear fits had a lower standard error, the uncertainty in capacity and EER for a given evaporator temperature was also low.

We demonstrated that EVAP-COND can be used effectively in developing evaporator capacity correlations, which will facilitate the use of the C-B Method.

The C-B Method does not have any inherent features that would produce a bias in predicting $Q(95)$ and $\operatorname{EER}(82)$ values. The values obtained in this study for $Q(95)$ and EER(82), under predictions or over predictions, are caused by random deviations between the obtained system test results and evaporator and condensing unit performance correlations.

Predicted values of SEER have strong under predicting tendencies due to the conservative $99^{\text {th }}$ percentile selection of the cyclic degradation coefficient. The category of equipment involved in this study can have a $C_{D}$ in the range from 0.09 to 0.25 , with 0.24 being the $99^{\text {th }}$ percentile value that was used in our SEER calculations. It appears that there is no other way to improve SEER predictions other than providing the matched system $C_{D}$ value along with condensing unit performance correlations for the application of the C-B Method. It is reasonable to assume that a mixed system would have a $C_{D}$ very similar to that of the matched system if only the evaporator and indoor fan are the replaced system components. 
As compared to the traditional approach for rating mixed systems that is based on the matched system $Q(95)$ and SEER values and adjusting them using coil capacity ratios (or similar scaling parameters), the C-B Method is an inherently more accurate methodology; the selected prediction problems encountered in this study should be studied further to tighten this procedure.

Some of the procedural issues that must be stipulated before a working standard is produced include the following:

1) Standard superheats for evaporator curve development with a method to accommodate different superheat for evaporator capacity determination

2) Liquid line temperatures for evaporator curve development

3) Cyclic degradation coefficients for comparable systems with different expansion devices

4) Cyclic degradation coefficient for matched system provided to coil manufacturers, or a method developed to determine a default value

5) Procedure for developing CD Unit curves; it should include a method for accommodating different superheats at the evaporator exit

As an alternative to the C-B Method, the traditional method for testing mixed systems could be revisited if the evaporator saturation temperature from the $Q(95)$ matched system test was made available for the mixed system rater. This would facilitate an accurate estimation of the capacity ratio of the mixed and matched coils, which is used as the most influential scaling factor in the traditional rating method. This methodology does not require the matched system $C_{D}$ since it is included in the matched system SEER, which is available for rating. 


\section{REFERENCES}

ASHRAE 1988. ANSI/ASHRAE Standard 37. Methods of testing for rating unitary air conditioning and heat pump equipment. American Society of Heating, Refrigerating and Air-Conditioning Engineers. 1791 Tullie Circle NE, Atlanta, GA, USA.

ARI 2003. Standard 210/240, Standard for unitary air-conditioning and air-source heat pump equipment, Air-Conditioning and Refrigeration Institute, 4100 North Fairfax Drive, Suite 200, Arlington, VA 22203.

CFR, 2004a. Code of Federal Regulations, Part 430, Appendix M to Subpart B, Uniform test method for measuring the energy consumption of central air conditioners, Office of the Federal Register, National Archives and Records Administration, Washington, DC.

CFR, 2004b. Code of Federal Regulations, Part 430.24, Subsection m1, Units to be tested, Federal Register, National Archives and Records Administration, Washington, DC.

Coleman, H.W. and Steele, W.G., 1989. Experimentation and uncertainty analysis for engineers, John Wiley and Sons, New York, NY USA.

Domanski, P.A., 1989. Rating procedure for mixed air-source unitary air conditioners and heat pumps operating in the cooling mode - revision 1, NISTIR 89-4071, U. S. Dept of Commerce, Natn'l Institute of Standards and Technology, Gaithersburg, Maryland USA 20899.

Domanski, P.A., 1999. "Finned-Tube Evaporator Model With a Visual Interface", $20^{\text {th }}$ Int. Congress of Refrigeration, Sydney, Australia, September 19-24, 1999, International Institute of Refrigeration, Paris.

Dougherty, B., 2004. Personal communications, National Institute of Standards and Technology, Gaithersburg, Maryland, USA, 20899.

Graybill, F. A. and lyer, H. K., 1994. Regression Analysis: Concepts and Applications, Duxbury Press, Wadsworth Publishing Company, Belmont, California USA 94002, pp. 194-209.

Lemmon, E. W., McLinden, M. O., and Huber, M. L., 2002. NIST Standard Reference Database 23: NIST Reference Fluid Thermodynamic and Transport Properties REFPROP, Version 7, U. S. Dept of Commerce, Natn'l Institute of Standards and Technology, Gaithersburg, Maryland USA 20899.

Ott, Lyman. 1984. An introduction to statistical methods and data analysis, $2^{\text {nd }}$ edition, Duxbury Press, Boston, Massachusetts USA, pp. 280-324.

Moffat, R. 1988. Describing the uncertainties in experimental results. Experimental Thermal and Fluid Science. Vol. 1. pp. 3-17. 
Neter J., Wasserman W., and Kutner M.H., 1990. Applied linear statistical models, regression, analysis of variance and experimental design, $3^{\text {rd }}$ edition, Irwin publishing, Burr Ridge, Illinois, USA, pp.10.

Taylor, B. N., and Kuyatt, C. E., 1994. "Guidelines for evaluating and expressing the uncertainty of NIST measurement results", NIST Technical Note 1297, 1994 edition, U.S. Department of Commerce. 


\section{APPENDIX A: EVAPORATOR COIL DESCRIPTIONS}

Appendix A presents design information for the nine mixed evaporators tested at NIST. It includes a picture, design data, and refrigerant circuitry representation in the input format of the EVAP-COND simulation package. General information and name designation for the mixed evaporators is given in Table 2.1.

\section{Coil 1}
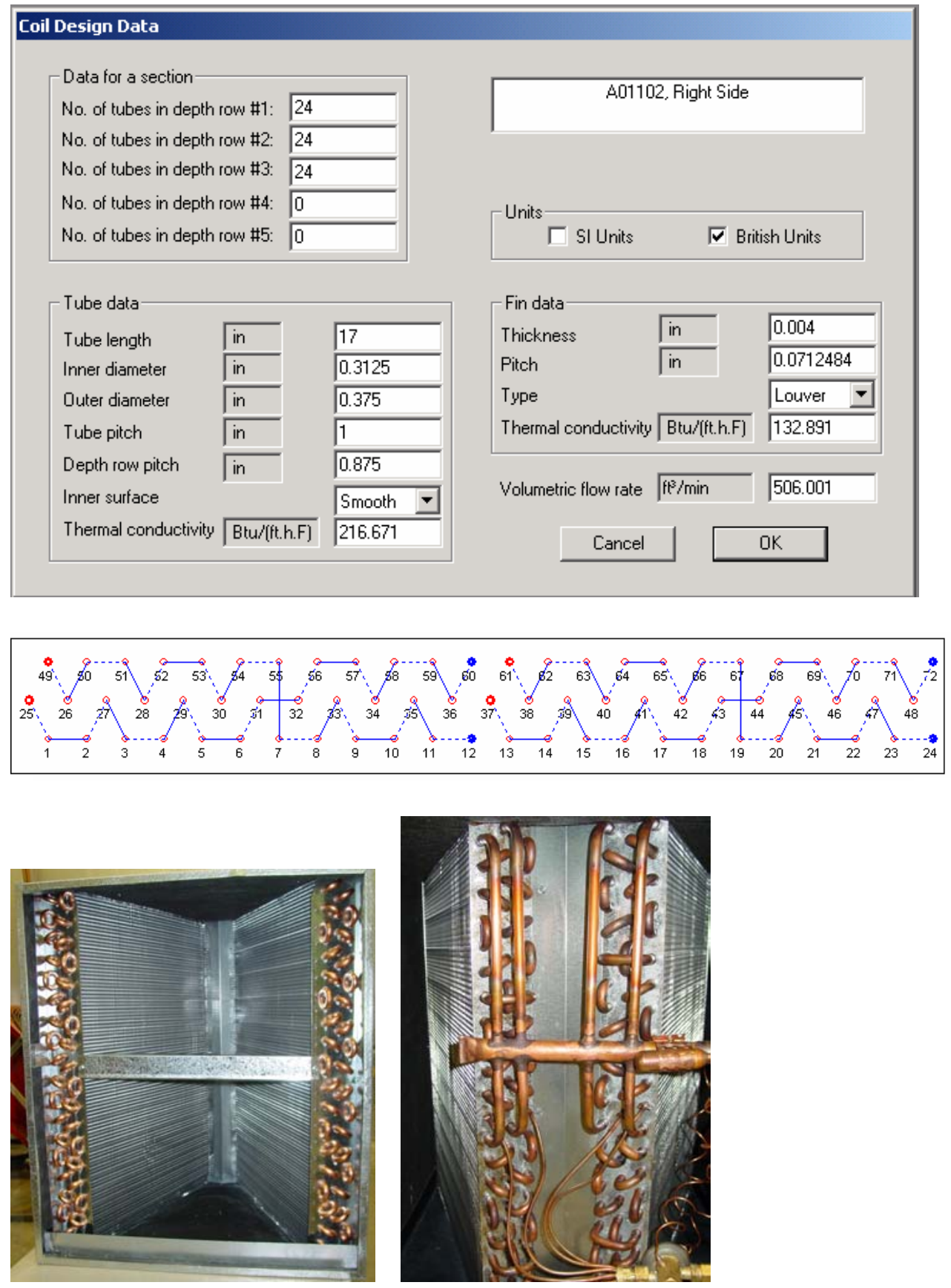


\section{Coil 2}

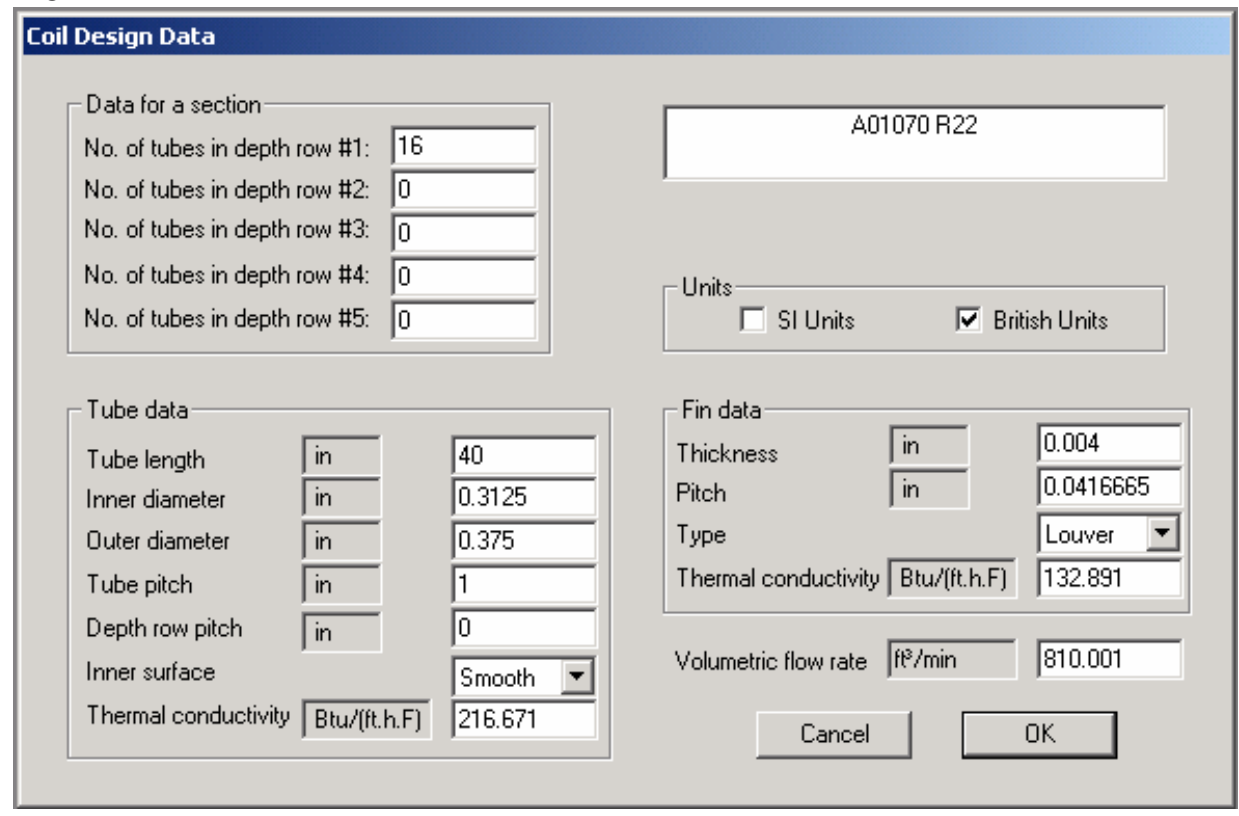

\section{Coil circuitry}
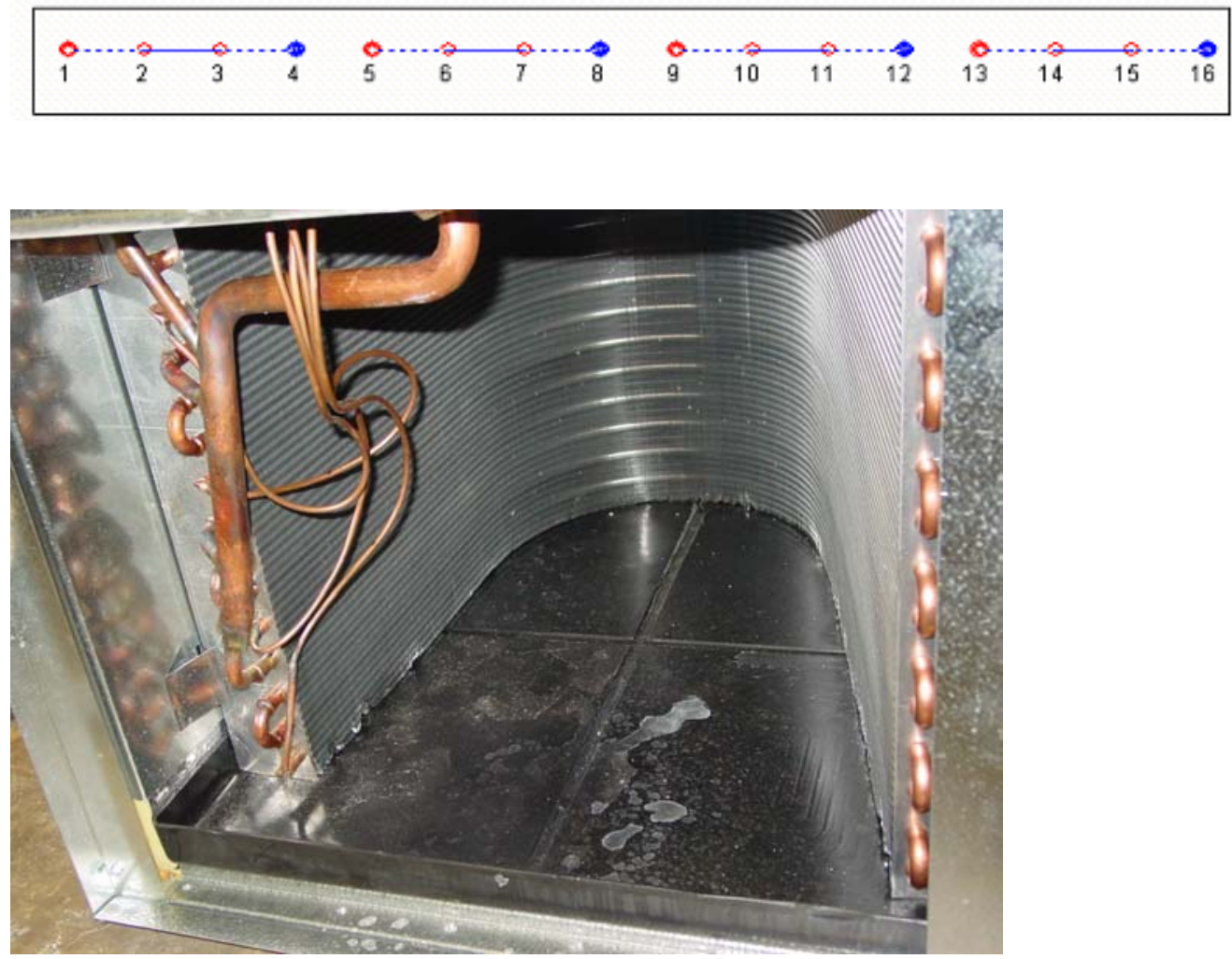


\section{Coil 3}

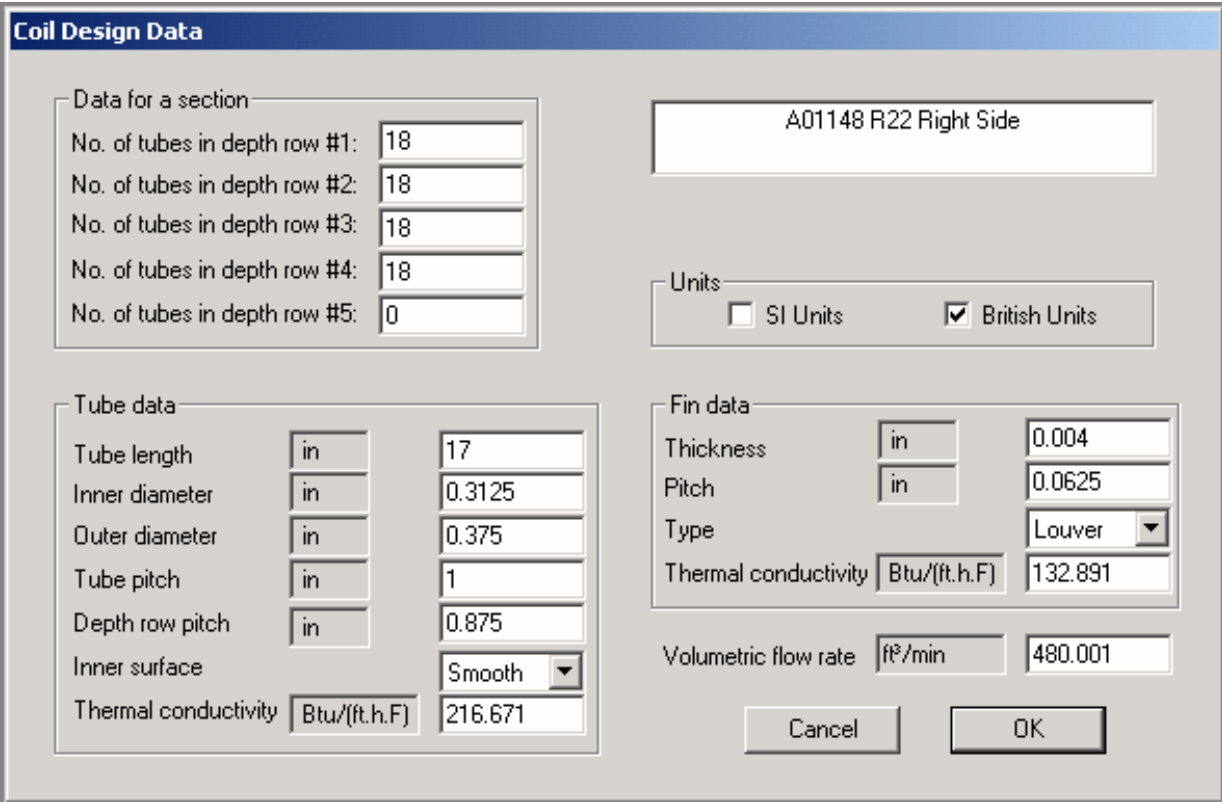

\section{Coil Circuitry (left slab)}
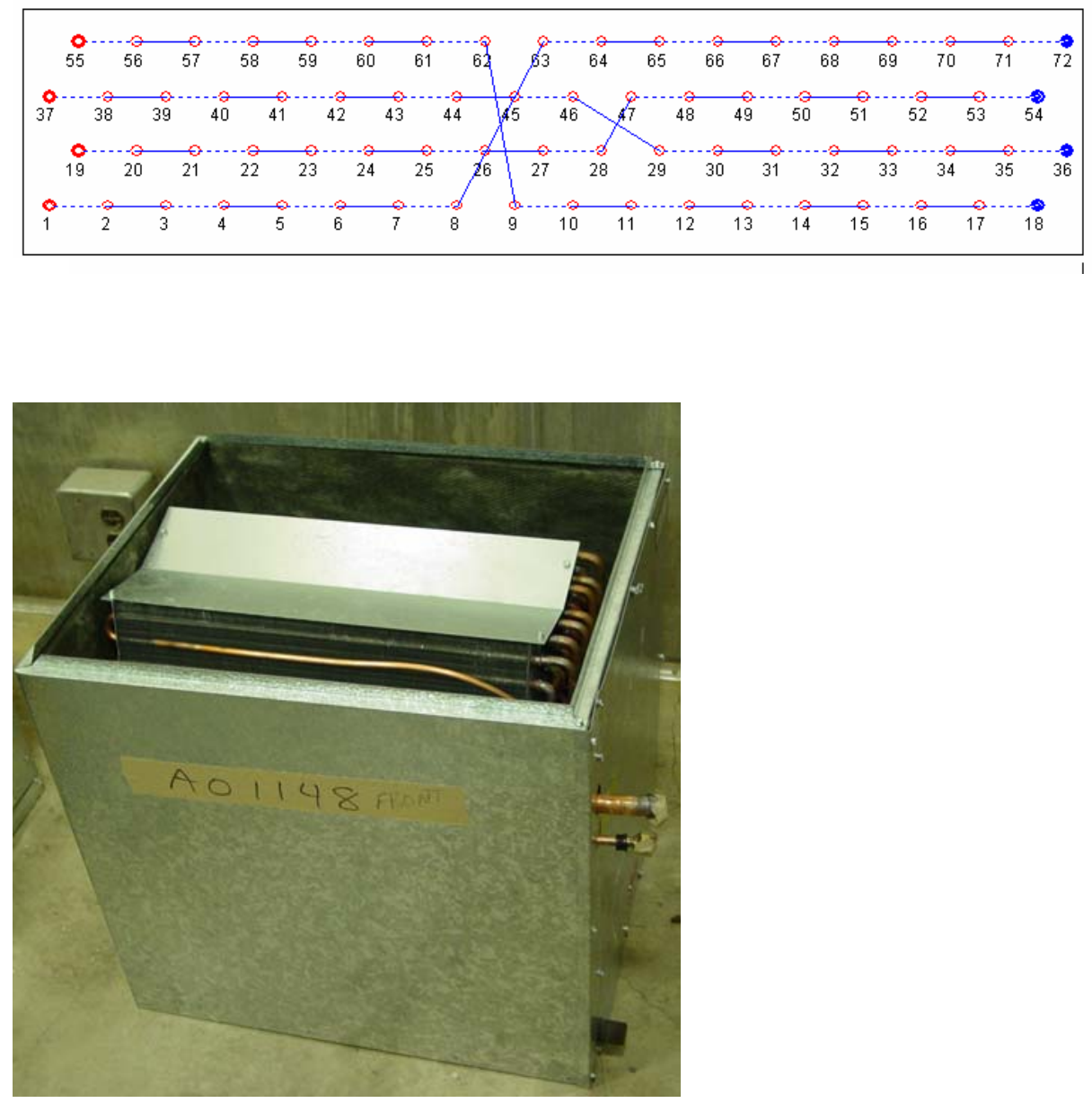


\section{Coil 4}

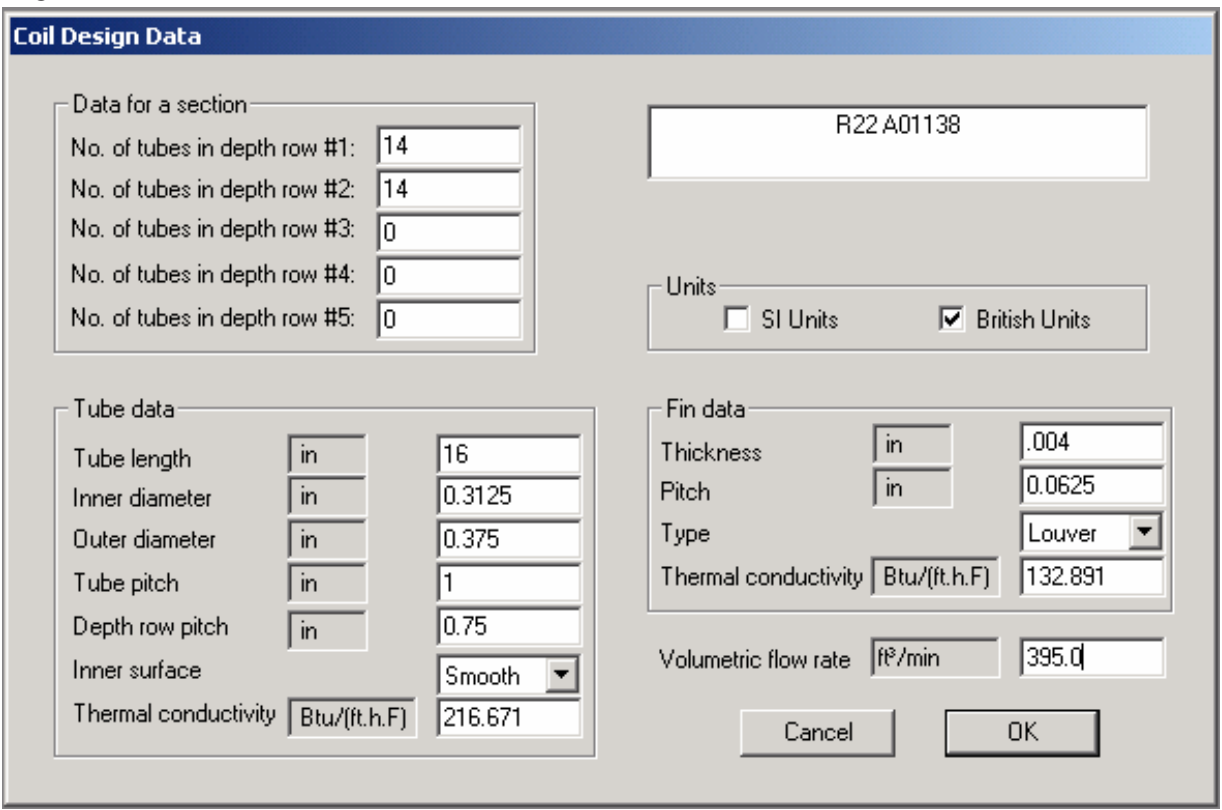

\section{Coil Circuitry (right slab as seen below)}
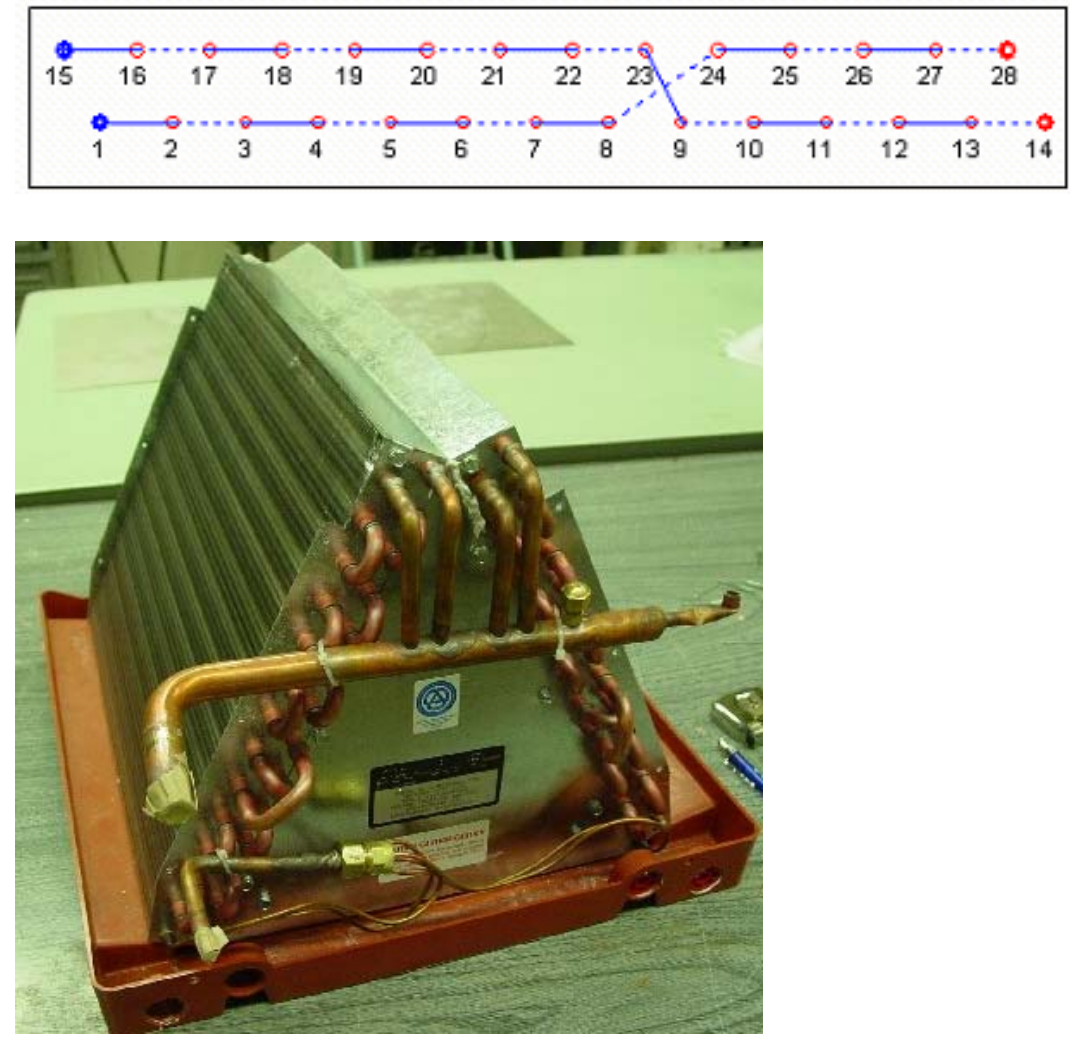


\section{Coil 5}

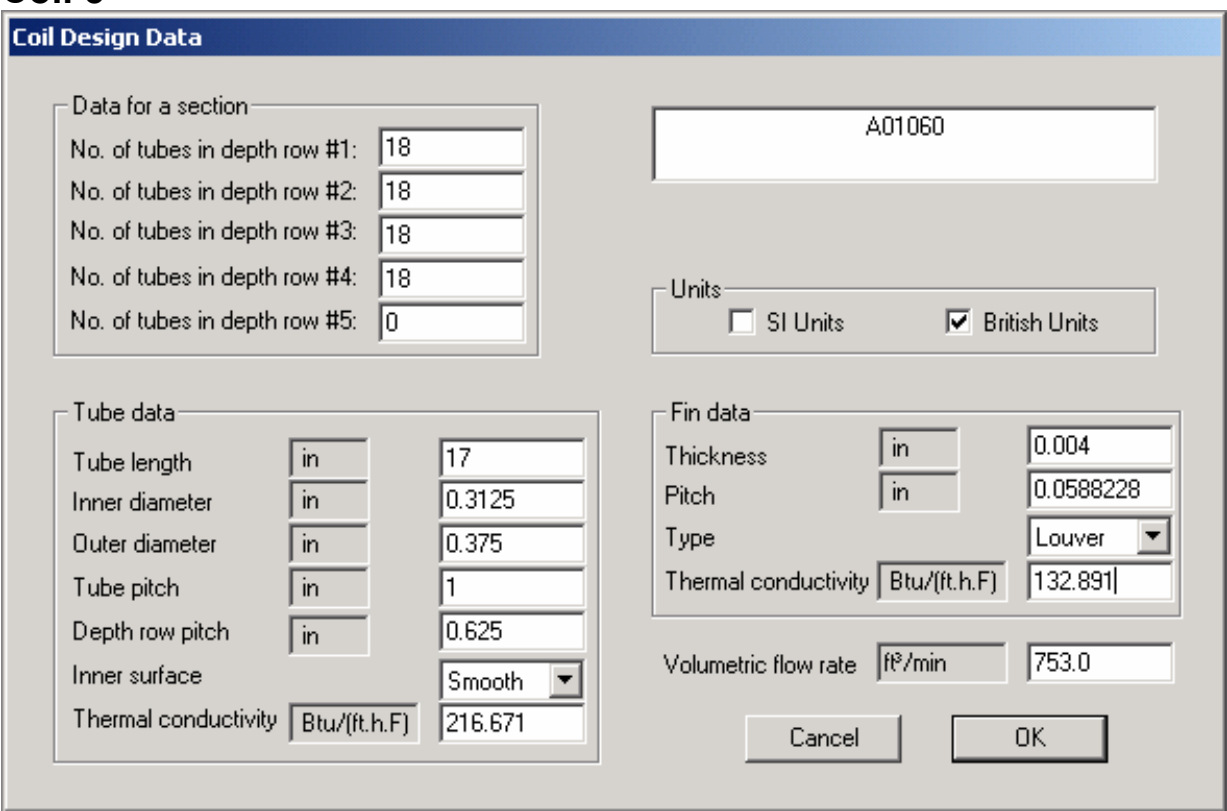

\section{Coil circuitry}
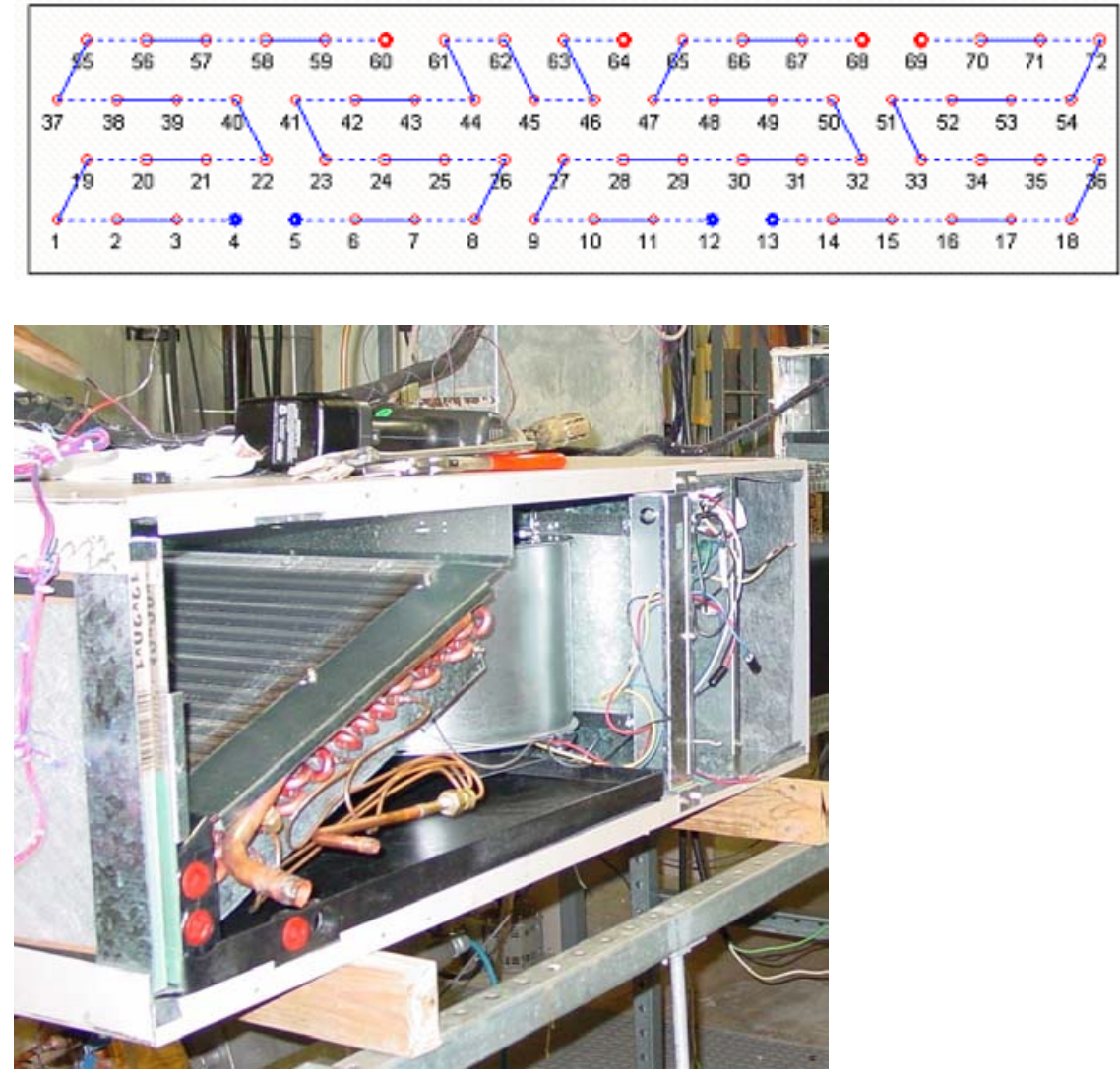


\section{Coil 6}

\section{Coil Design Data}

- Data for a section

No. of tubes in depth row $\# 1$ : 16

No. of tubes in depth row $\# 2$ : 16

No. of tubes in depth row \#3:

No. of tubes in depth row \#4:

No. of tubes in depth row \#5:

No. of tubes in depth row $\# 6$ : 16

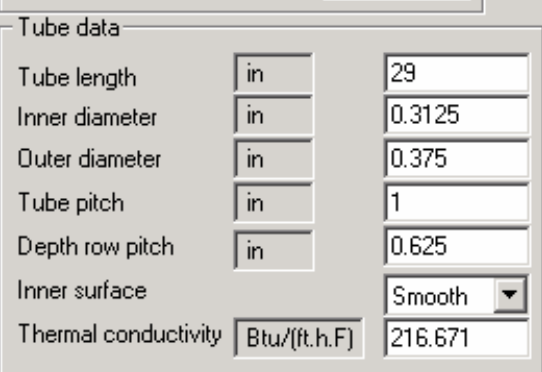
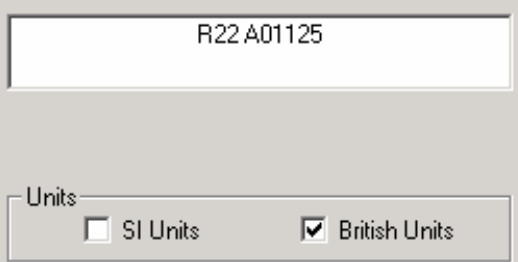

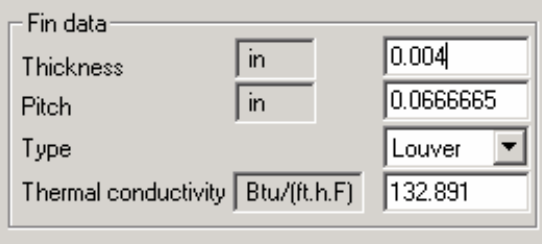

Volumetric flow rate $\longdiv { \mathrm { ft } ^ { 3 } / \mathrm { min } } \overline { 5 7 5 . 0 0 1 }$

Cancel

OK

\section{Coil circuitry}
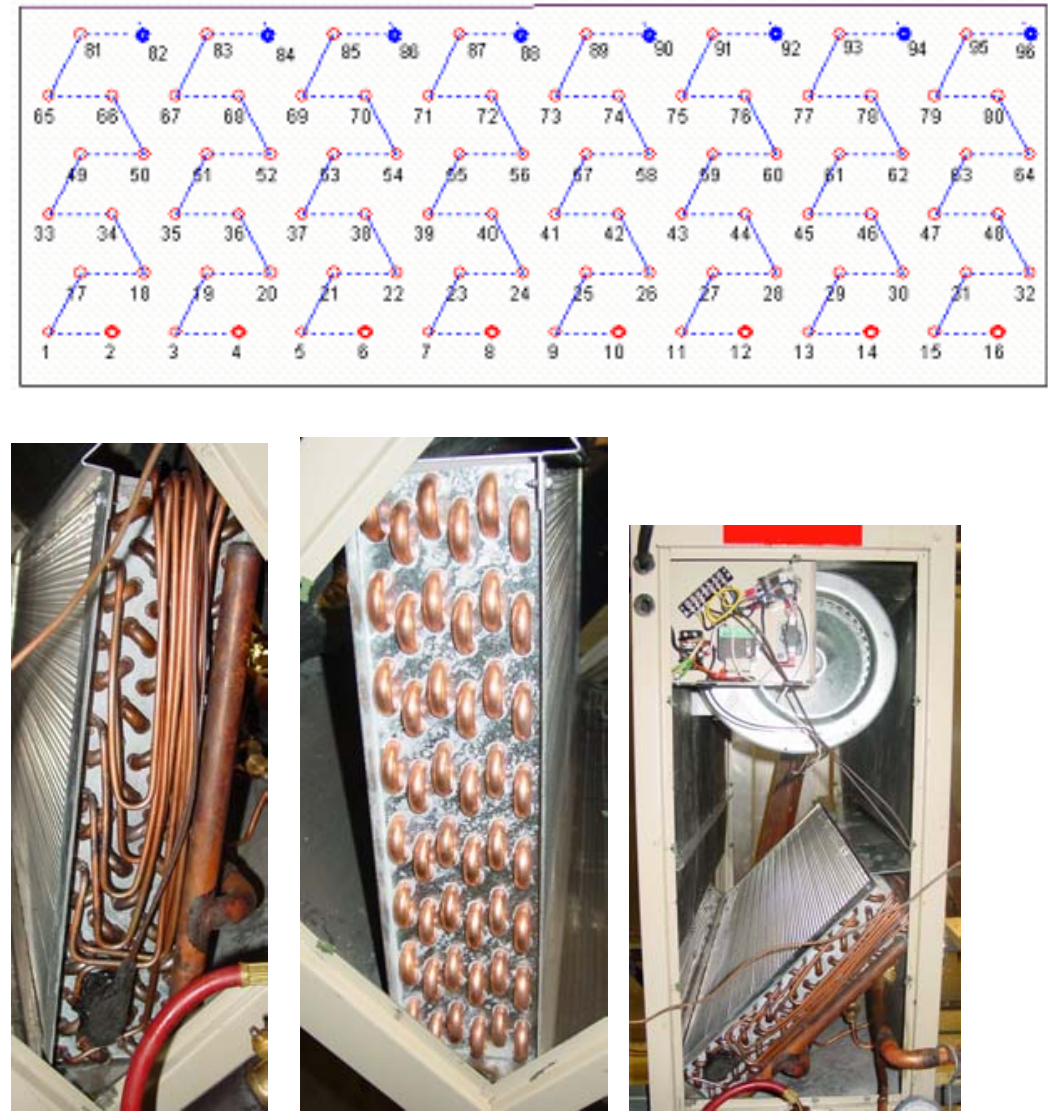


\section{Coil 7}

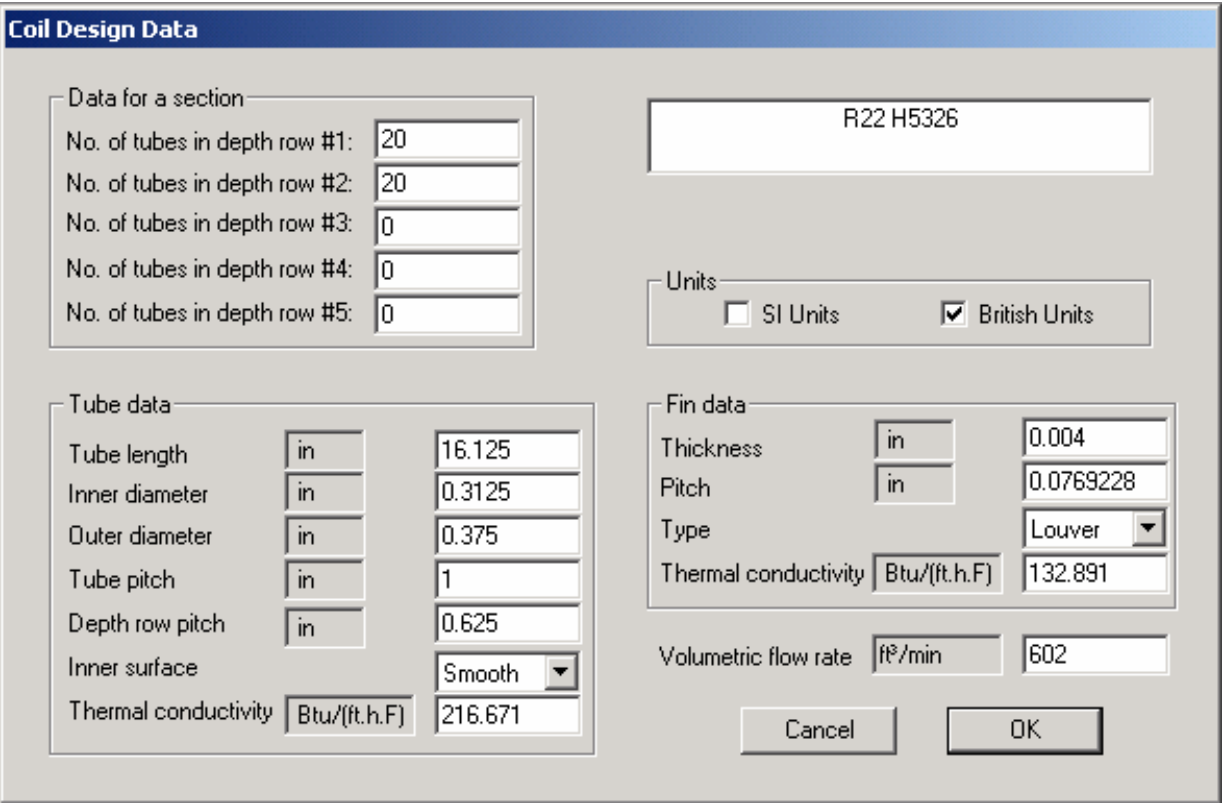

\section{Coil circuitry (left or right slab)}
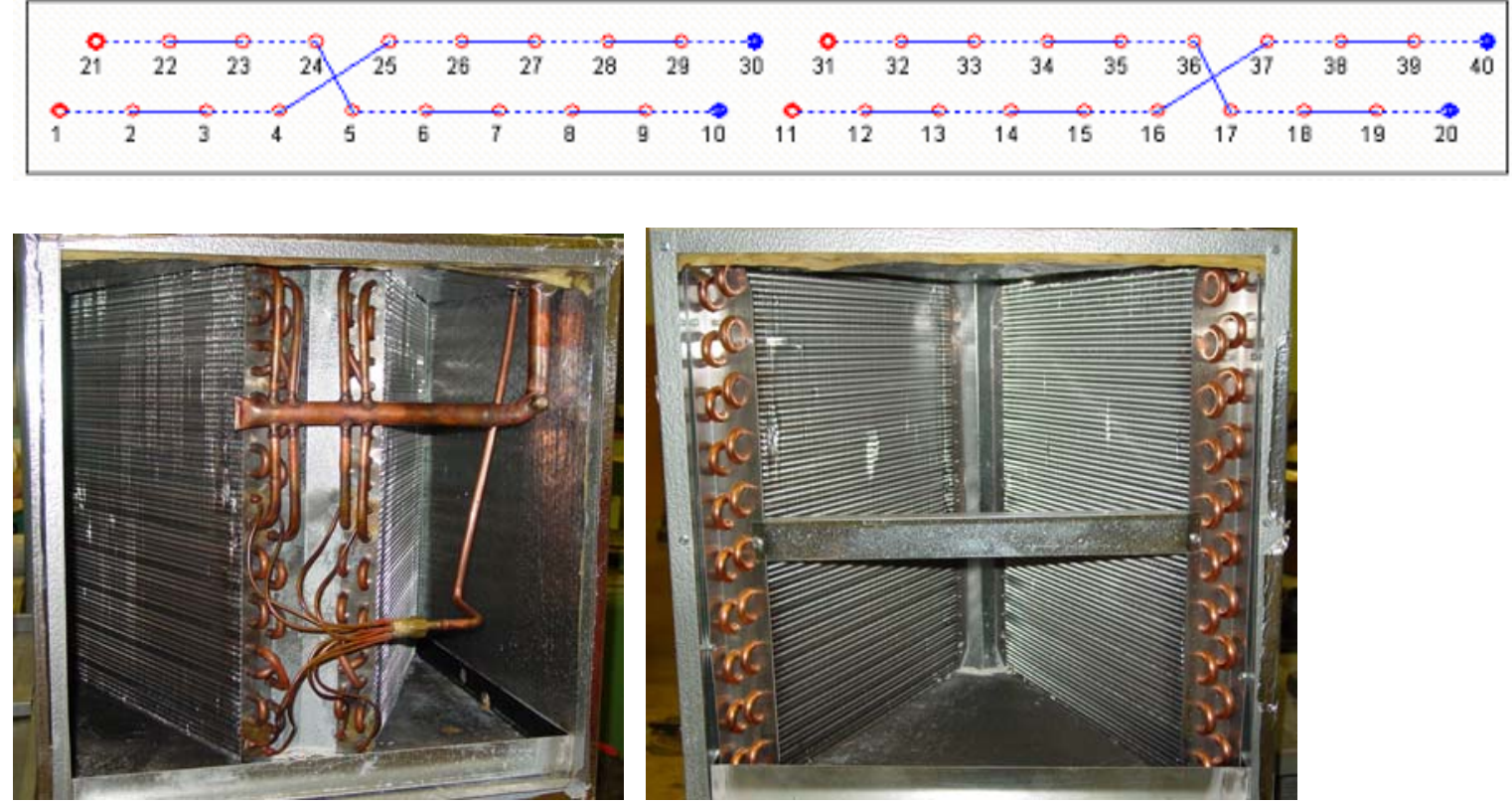


\section{Coil 8}

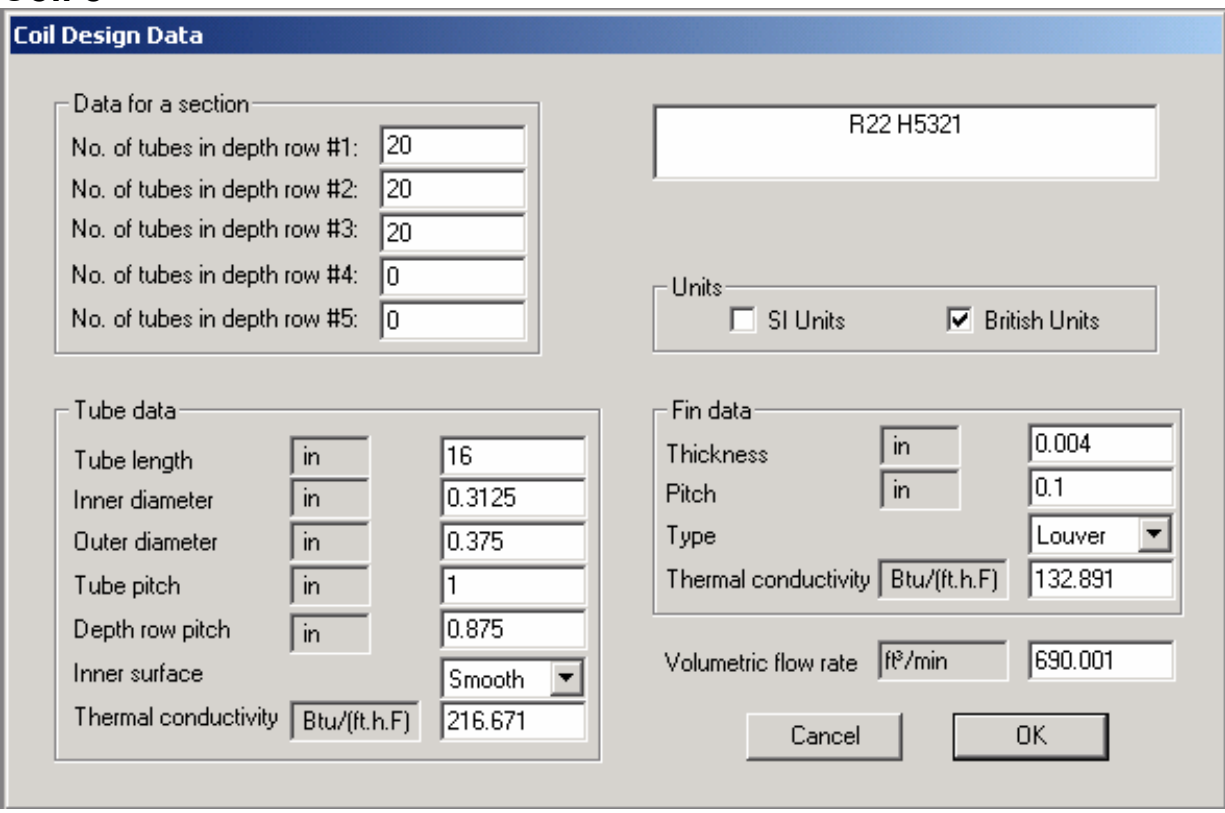

\section{Coil circuitry (left slab)}
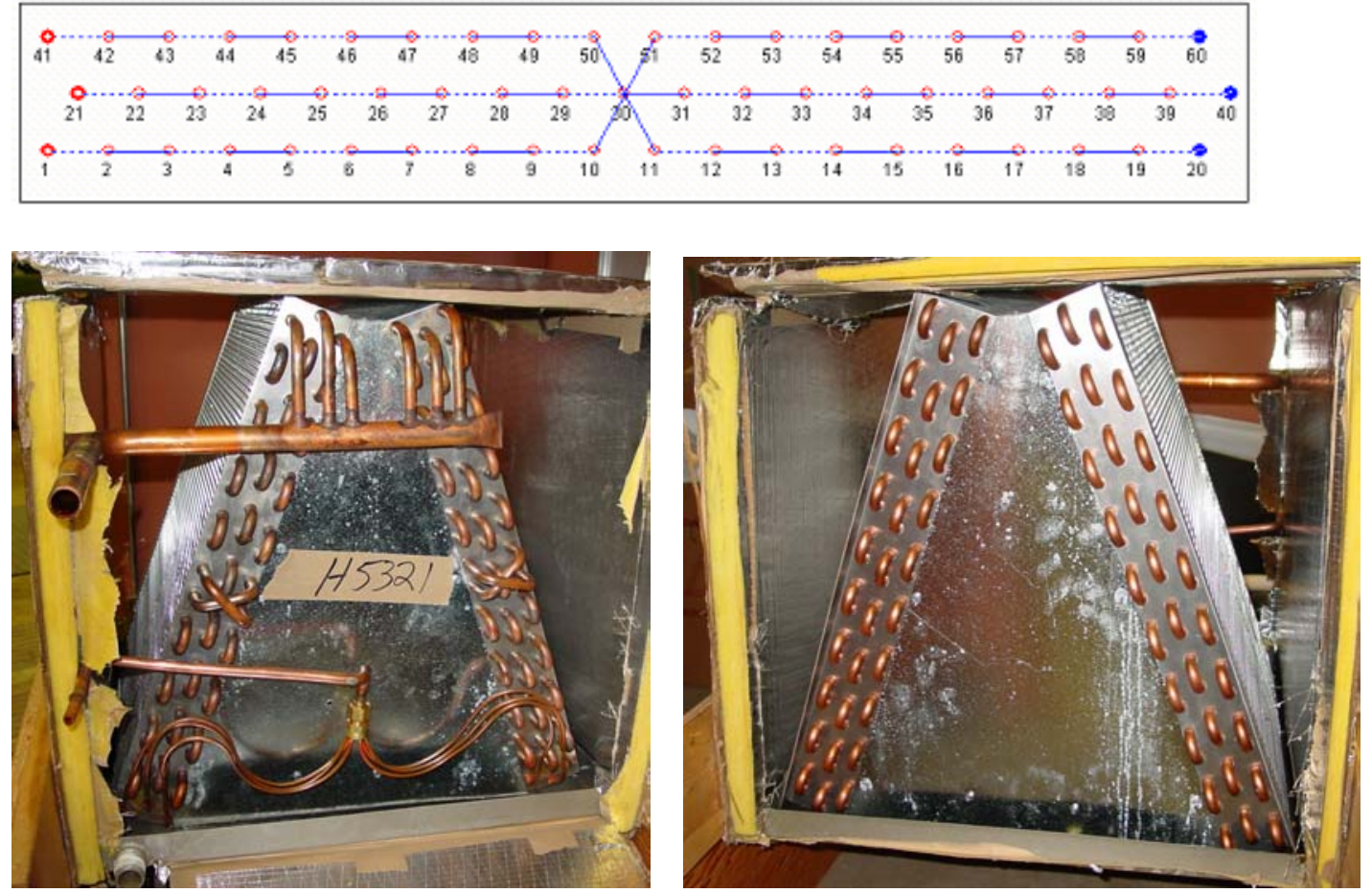


\section{Coil 9}

\section{Coil Design Data}

- Data for a section

No. of tubes in depth row \#1: 15

No. of tubes in depth row $\# 2$ : 15

No. of tubes in depth row $\# 3$ :

No. of tubes in depth row \#4: 15

No. of tubes in depth row \#5: 0

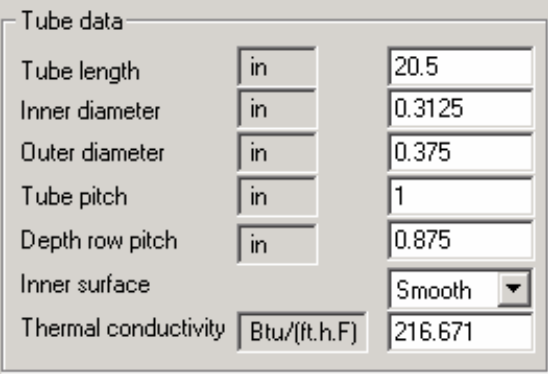
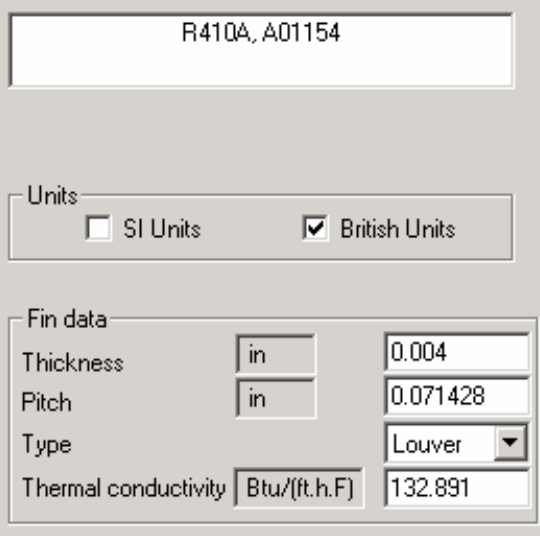

Volumetric flow rate $\longdiv { \mathrm { ft } ^ { 3 } / \mathrm { min } } \longdiv { 5 0 0 }$

Cancel $\quad$ OK

\section{Coil circuitry}
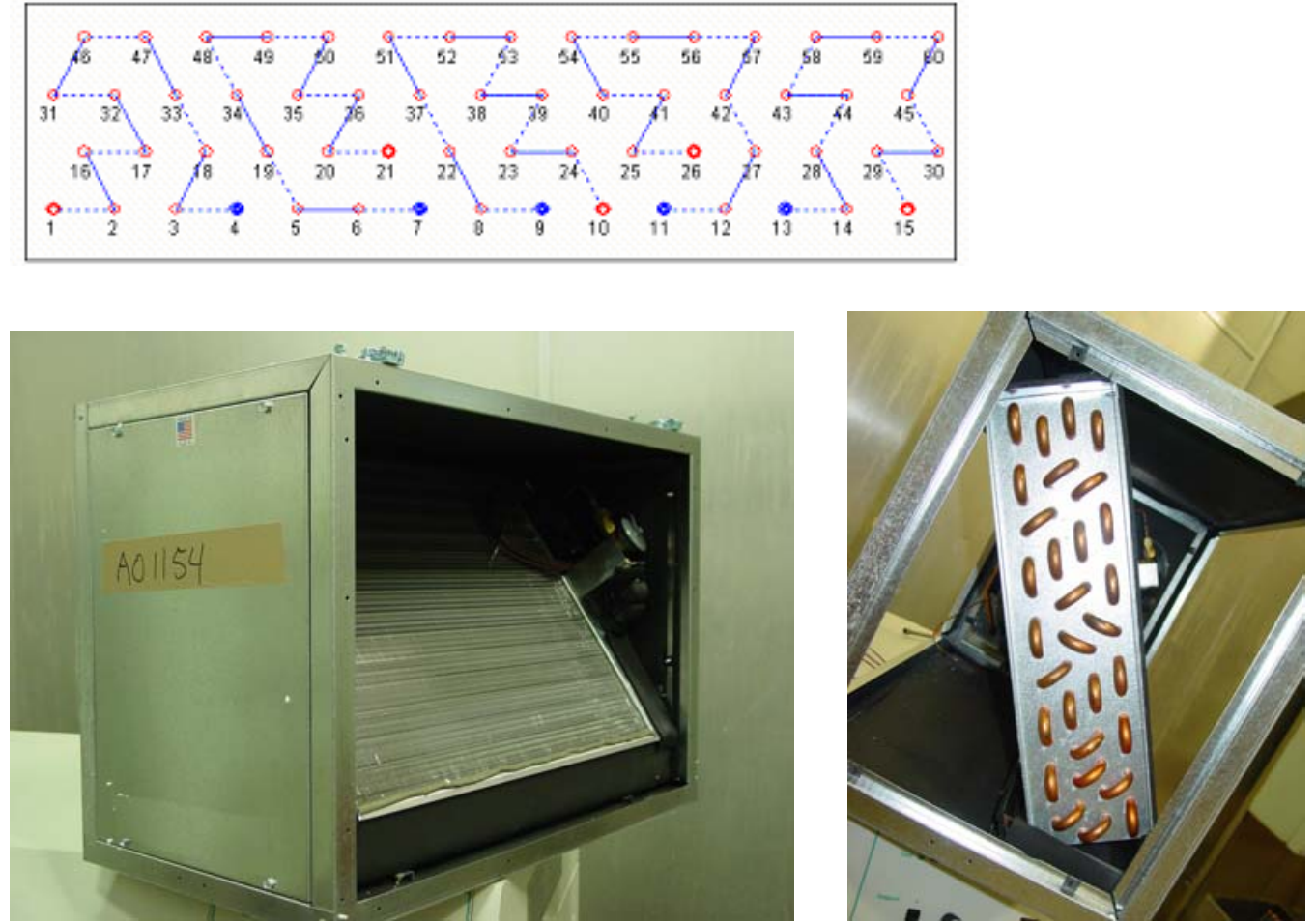


\section{APPENDIX B: UNCERTAINTY ANALYSIS}

\section{A. 1 General Remarks}

The uncertainty analysis was performed to gain knowledge about the uncertainty of the measured and calculated data. This Appendix presents the major equations used for the uncertainty analysis.

\section{A. 2 Theory}

The uncertainty of a quantity $R$ calculated from $n$ independent measurements $x_{\mathrm{i}}$ is a function of the individual uncertainty of each measurement.

$$
R=f\left(x_{1}, x_{2}, x_{3}, \ldots, x_{n}\right)
$$

When each measurement, $x_{\mathrm{i}}$, has a given uncertainty, $d x_{\mathrm{i}}$, the maximum uncertainty of $R$ is given by:

$$
E_{\mathrm{R}}=\left|\frac{\partial f}{\partial \mathrm{x}_{1}} \mathrm{~d} x_{1}\right|+\left|\frac{\partial}{\partial \mathrm{x}_{2}} \mathrm{~d} x_{2}\right|+\left|\frac{\partial}{\partial x_{3}} \mathrm{~d} x_{3}\right|+\ldots . .+\left|\frac{\partial}{\partial x_{\mathrm{n}}} \mathrm{d} x_{\mathrm{n}}\right| .
$$

However, using the maximum error to judge the uncertainty of a calculated quantity is not common. Usually the standard deviation (root sum square) is regarded to be a much better approach to a quantity's uncertainty.

$$
E_{\mathrm{R}}=\sqrt{\left(\frac{\partial}{\partial x_{1}} \mathrm{~d} x_{1}\right)^{2}+\left(\frac{\partial}{\partial x_{2}} \mathrm{~d} x_{2}\right)^{2}+\left(\frac{\partial f}{\partial x_{3}} \mathrm{~d} x_{3}\right)^{2}+\ldots . .+\left(\frac{\partial}{\partial x_{\mathrm{n}}} \mathrm{d} x_{\mathrm{n}}\right)^{2}}
$$

The absolute error calculated with equation (A.3) is often converted to a relative error having the units of percent.

$$
e_{\mathrm{R}}=\frac{E_{\mathrm{R}}}{R} 100
$$

\section{A. 3 Temperature Measurements}

Most of the temperature measurements performed for these tests were determined by thermocouples. Their voltage signals were measured with the data acquisition system and then converted into a temperature.

The equation used in the test rig's control program to convert the voltage signals into temperatures was a sixth degree polynomial of the form:

$$
\vartheta=f(V)=\frac{9}{5}\left(A+B V+C V^{2}+D V^{3}+E V^{4}+F V^{5}+G V^{6}\right)+32
$$


where:

$$
\begin{array}{lll}
\vartheta & = & \text { temperature }\left({ }^{\circ} \mathrm{F}\right) \\
\mathrm{V} & = & \text { measured voltage }(\mu \mathrm{V})
\end{array}
$$

If one premises that the uncertainty of the equation itself can be neglected, only one derivation is needed to evaluate the uncertainty in the temperature measurements.

$$
\frac{\partial \vartheta}{\partial V}=\frac{9}{5}\left(B+2 C V+3 D V^{2}+4 E V^{3}+5 F V^{4}+6 G V^{5}\right)
$$

According to the manufacturer of the voltmeter, the $95 \%$ uncertainty of the voltage measurement $(V M)$ was: $E_{V M}=d V(V M)= \pm 0.007 \%$ of reading $+5 \mu \mathrm{V}$.

The measurement of a temperature $(\vartheta)$ actually is the measurement of the difference to a reference temperature. The data acquisition system provided temperature compensation to $0{ }^{\circ} \mathrm{C}\left(32^{\circ} \mathrm{F}\right)$ with a given uncertainty of: $\mathrm{E}_{\mathrm{TC}}=\mathrm{dTC}= \pm 0.2236^{\circ} \mathrm{C}= \pm 0.4025^{\circ} \mathrm{F}$.

Rewriting equation A.3 for the measurement of the absolute temperature gives:

$$
\begin{aligned}
& \vartheta=f(V) \\
& E_{\mathrm{T}}=\sqrt{\left(\frac{\partial \vartheta}{\partial V} \mathrm{~d} V M\right)^{2}+(\mathrm{d} T C)^{2}}
\end{aligned}
$$

In addition to the common thermocouple measurements, the dew-point temperature in the air duct was measured to evaluate the humidity ratio of the moist air in the duct.

The manufacturer of the dew-point hygrometer specified the $95 \%$ uncertainty in this measurement to be: $E_{T_{\text {dew }}}=\mathrm{d} T_{\text {dew }}= \pm 0.05 \%$ of reading .

\section{A. 4 Temperature Difference Measurements}

The evaluation of the uncertainty of a temperature difference $(\Delta \vartheta)$ measurement using a thermopile is slightly more complicated than that for a normal temperature measurement. The uncertainty evaluation is presented using the air duct temperature difference as an example, because this shows the most complicated case. Again there are two independent uncertainties being part of the measurement uncertainty. The first is the uncertainty caused by the voltage signal measurement, discussed in section A.3. The cause for the second uncertainty influencing the measurement of a temperature difference is the nonlinear character of the temperature/voltage function (see equation A.5). The nonlinearity requires temperature at one end of the thermopile used for the temperature difference measurement to be known.

The temperature difference across the indoor coil was calculated using both the voltage signals of the temperature difference measurement $(\Delta V)$ and the average voltage signal 
$\left(V_{\text {av. }}\right)$ of the entering temperature measurement of the air duct. The equation used to do so was:

$$
\Delta \vartheta=f\left(V_{\mathrm{av} .}+\Delta V\right)-f\left(V_{\mathrm{av} .}\right)
$$

The entering temperature was measured using 25 thermocouples equally distributed over the air duct's cross section. The average of the 25 temperature signals was considered to be the entering temperature. For the uncertainty in this average entering temperature the average voltage measurement uncertainty $E_{V M \text {,av. }}$ of the 25 measurements was calculated.

$$
E_{V M, \mathrm{av} .}=\mathrm{d} V_{\mathrm{av}}(V M)=\sum_{\mathrm{x}=1}^{25} \frac{\mathrm{d} V_{\mathrm{av} .}\left(V M_{\mathrm{x}}\right)}{25}
$$

All 25 thermocouples were connected to the same temperature compensation. This means the overall uncertainty of the air's average entering temperature voltage signal $V_{a v}$. was:

$$
d V_{\text {av. }}=\sqrt{E_{V M, a v .}^{2}+E_{T C}^{2}}=\sqrt{\left(d V_{\text {av. }}(V M)\right)^{2}+\left(\mathrm{d} V_{\text {av. }}(T C)\right)^{2}}
$$

To evaluate equation A.11 the uncertainty in the temperature compensation must be rewritten to have the unit of $\mu \mathrm{V}$. Using equation $\mathrm{A} .5$ one finds that an uncertainty of $E_{T C}=\mathrm{d} T C= \pm 0.2236^{\circ} \mathrm{C}= \pm 0.4025^{\circ} \mathrm{F}$ in the temperature compensation to $0{ }^{\circ} \mathrm{C}\left(32{ }^{\circ} \mathrm{F}\right)$ is equivalent to a voltage signal uncertainty of $\mathrm{d} V_{\mathrm{av}}(T C)= \pm 8.6264 \mu \mathrm{V}$. As already mentioned, the uncertainty of the voltage signal measurement was given from manufacturer data.

The nonlinearity of the voltage/temperature function (A.5) causes an uncertainty dslope in the temperature difference that depends on the uncertainty in the entering temperature voltage signal $V_{\text {av. }}$.

$$
\begin{aligned}
E_{\text {slope }}=\mathrm{d} \text { slope }=\mid( & \left({ }\left(V_{\mathrm{av} .}+d V\right)-\vartheta\left(V_{\mathrm{av} .}\right)\right) \\
& -\left(\vartheta\left(V_{\mathrm{av} .}+\mathrm{d} V_{\mathrm{av} .}+\Delta V\right)-\vartheta\left(V_{\mathrm{av} .}+\mathrm{d} V_{\mathrm{av} .}\right)\right) \mid
\end{aligned}
$$

where:

$$
\begin{array}{lll}
V_{\mathrm{av} .} & = & \text { entering temperature voltage signal }(\mu V) \\
\mathrm{d} V_{\mathrm{av}}= & \text { uncertainty of the entering temperature voltage signal }(\mu V) \\
\Delta V= & \text { temperature difference voltage signal }(\mu V)
\end{array}
$$

Remembering that an additional uncertainty in the temperature difference is caused by the voltage measurement of the temperature difference voltage signal $(\Delta V)$, the uncertainty of the air duct temperature difference is given to be: 


$$
E_{\Delta \vartheta}=\mathrm{d} \Delta \vartheta=\left[\left(\frac{\partial \vartheta}{\partial V} \mathrm{~d} \Delta V\right)^{2}+\mathrm{d} s / o p e^{2}\right]^{1 / 2}
$$

\section{A. 5 Uncertainty of the Air Side Capacity}

The air side capacity of the heat pump was evaluated using the equation:

$$
\dot{Q}_{C}=\dot{Q}_{S}+\dot{Q}_{L}
$$

where:

$$
\begin{array}{lll}
\dot{Q}_{\mathrm{S}} & = & \text { sensible capacity, } \mathrm{kW}(B t u / h) \\
\dot{Q}_{\mathrm{L}} & = & \text { latent capacity, } \mathrm{kW}(B t u / h)
\end{array}
$$

The sensible capacity is the heat needed to cool or heat the moist air passing the heat pump's indoor coil. The latent capacity is the heat rejected by water vapor condensing on the air coil. Condensation does not occur in the heating mode.

The two different capacities were calculated separately and then added (A.14). Therefore the uncertainty of the air-side capacity can be written as:

$$
E_{\dot{Q}_{C}}=\left[\left(\frac{\partial \dot{Q}_{C}}{\partial \dot{Q}_{S}} \mathrm{~d} \dot{Q}_{S}\right)^{2}+\left(\frac{\partial \dot{Q}_{C}}{\partial \dot{Q}_{L}} \mathrm{~d} \dot{Q}_{\mathrm{L}}\right)^{2}\right]^{1 / 2}=\left(\mathrm{d} \dot{Q}_{S}{ }^{2}+\mathrm{d}_{\dot{Q}_{L}}{ }^{2}\right)^{1 / 2}
$$

The equations for both the sensible and latent capacities and their uncertainties are presented on the following pages.

\section{A. 5. 1 Uncertainty of the Sensible Capacity}

According to ASHRAE Standard $116-1993$ the sensible capacity $Q_{\mathrm{S}}$ is given by:

$$
\dot{Q}_{\mathrm{S}}=3600 C_{\mathrm{D}} A_{\mathrm{n}}\left(0.24+0.444 W_{\mathrm{av} .}\right)\left(\vartheta_{\mathrm{l}}-\vartheta\right)_{\mathrm{e}}\left[\frac{2 g_{\mathrm{C}} \Delta p_{\mathrm{n}} \rho_{\mathrm{nact}}}{144\left(1-\beta^{2}\right)}\right]^{1 / 2}
$$

where:

$$
\begin{array}{lll}
C_{\mathrm{D}}= & \text { nozzle discharge coefficient }(0.986) \\
A_{\mathrm{n}}= & \text { nozzle throat area, } \mathrm{m}^{2}\left(f t^{2}\right) \\
W_{\mathrm{av} .}= & \left(W_{\mathrm{e}}+W_{1}\right) / 2 \text { average humidity ratio, } \mathrm{kg} \mathrm{H} \mathrm{H}_{2} \mathrm{O} / \mathrm{kg} \text { dry air } \\
& \left(\mathrm{lb} \mathrm{H}_{2} \mathrm{O} / \mathrm{lb} \text { dry air }\right) \\
\vartheta_{\mathrm{l}}-\vartheta_{\mathrm{e}}= & \text { indoor coil air temperature rise, }{ }^{\circ} \mathrm{C}\left({ }^{\mathrm{O}} \mathrm{F}\right) \\
g_{\mathrm{C}}= & \text { gravity constant }\left(32.174 \mathrm{ft} \cdot l b_{\mathrm{m}} / l b_{\mathrm{f}} \cdot \mathrm{s}^{2}\right) \\
\Delta p_{\mathrm{n}}= & \text { static pressure drop across nozzle, } \mathrm{kPa}(p s i a) \\
\rho_{\text {nact }}= & \text { density of the moist air, } \mathrm{kg} / \mathrm{m}^{3}\left(l b / f t^{3}\right)
\end{array}
$$




$$
\begin{array}{lll}
144 & = & \text { unit conversion factor from } \mathrm{in}^{2} \text { to } f t^{2} \\
\beta & = & \text { area relation factor }(0.250723)
\end{array}
$$

The partial derivatives required for the uncertainty analysis of $Q_{\mathrm{S}}$ are:

$$
\begin{aligned}
& \frac{\partial \dot{Q}_{\mathrm{S}}}{\partial \mathrm{A}_{\mathrm{n}}}=3600 C_{\mathrm{D}}\left(0.24+0.444 W_{\mathrm{av} .}\right)\left(\vartheta_{\mathrm{l}}-\vartheta_{\mathrm{e}}\right)\left[\frac{2 g_{\mathrm{C}} \Delta p_{\mathrm{n}} \rho_{\text {nact }}}{144\left(1-\beta^{2}\right)}\right]^{1 / 2} \\
& \frac{\partial \dot{Q}_{\mathrm{S}}}{\partial W_{\mathrm{e}}}=1800 C_{\mathrm{D}} A_{\mathrm{n}} 0.444\left(\vartheta_{\mathrm{l}}-\vartheta_{\mathrm{e}}\right)\left[\frac{2 g_{\mathrm{C}} \Delta p_{\mathrm{n}} \rho_{\text {nact }}}{144\left(1-\beta^{2}\right)}\right]^{1 / 2} \\
& \frac{\partial \dot{Q}_{\mathrm{S}}}{\partial W_{1}}=1800 C_{\mathrm{D}} A_{\mathrm{n}} 0.444\left(\vartheta_{\mathrm{l}}-\vartheta_{\mathrm{e}}\right)\left[\frac{2 g_{\mathrm{C}} \Delta p_{\mathrm{n}} \rho_{\text {nact }}}{144\left(1-\beta^{2}\right)}\right]^{1 / 2} \\
& \frac{\partial \dot{Q}_{\mathrm{S}}}{\partial\left(\vartheta_{\mathrm{l}}-\vartheta_{\mathrm{e}}\right)}=3600 C_{\mathrm{D}} A_{\mathrm{n}}\left(0.24+0.444 W_{\text {av. }}\right)\left[\frac{2 g_{\mathrm{C}} \Delta p_{\mathrm{n}} \rho_{\text {nact }}}{144\left(1-\beta^{2}\right)}\right]^{1 / 2} \\
& \frac{\partial \dot{Q}_{S}}{\partial \Delta p_{\mathrm{n}}}=1800 C_{\mathrm{D}} A_{\mathrm{n}}\left(0.24+0.444 W_{\text {av. }}\right)\left(\vartheta_{\mathrm{l}}-\vartheta_{e}\right)\left[\frac{2 g_{\mathrm{C}} \rho_{\text {nact }}}{144\left(1-\beta^{2}\right) \Delta p_{\mathrm{n}}}\right]^{1 / 2} \\
& \frac{\partial \dot{Q}_{\mathrm{S}}}{\partial \rho_{\text {nact }}}=1800 C_{\mathrm{D}} A_{\mathrm{n}}\left(0.24+0.444 W_{\text {av. }}\right)\left(\vartheta_{\mathrm{l}}-\vartheta_{\mathrm{e}}\right)\left[\frac{2 g_{\mathrm{C}} \Delta p_{\mathrm{n}}}{144\left(1-\beta^{2}\right) \rho_{\text {nact }}}\right]^{1 / 2} \\
& \frac{\partial \dot{Q}_{\mathrm{S}}}{\partial \beta}=3600 C_{\mathrm{D}} A_{\mathrm{n}}\left(0.24+0.444 W_{\text {av. }}\right)\left(\vartheta_{\mathrm{l}}-\vartheta_{\mathrm{e}}\right) \beta\left[\frac{2 g_{\mathrm{C}} \Delta p_{\mathrm{n}} \rho_{\text {nact }}}{144\left(1-\beta^{2}\right)^{3}}\right]^{1 / 2}
\end{aligned}
$$

Using the above partial derivatives for rewriting equation A.3 gives:

$$
\begin{aligned}
E_{Q_{\mathrm{S}}}=\left[\left(\frac{\partial \dot{Q}_{\mathrm{S}}}{\partial A_{\mathrm{n}}} \mathrm{d} A_{\mathrm{n}}\right)^{2}+\left(\frac{\partial \dot{Q}_{\mathrm{S}}}{\partial W_{\mathrm{e}}} \mathrm{d} W_{\mathrm{e}}\right)^{2}+\left(\frac{\partial \dot{Q}_{\mathrm{S}}}{\partial W_{\mathrm{l}}} \mathrm{d} W_{\mathrm{l}}\right)^{2}+\left(\frac{\partial \dot{\mathbf{Q}}_{\mathrm{S}}}{\partial \Delta p_{\mathrm{n}}} \mathrm{d} \Delta p_{\mathrm{n}}\right)^{2}+\right. \\
\left.+\left(\frac{\partial \dot{Q}_{\mathrm{S}}}{\partial\left(\vartheta_{\mathrm{l}}-\vartheta_{\mathrm{e}}\right)} \mathrm{d}\left(\vartheta_{\mathrm{l}}-\vartheta_{\mathrm{e}}\right)\right)^{2}+\left(\frac{\partial \dot{\mathbf{Q}}_{\mathrm{S}}}{\partial \rho_{\text {nact }}} \mathrm{d} \rho_{\text {nact }}\right)^{2}+\left(\frac{\partial \dot{\mathrm{Q}}_{\mathrm{S}}}{\partial \beta} \mathrm{d} \beta\right)\right]^{1 / 2}
\end{aligned}
$$

Equation A.24 can be evaluated to give the uncertainty of $\dot{Q}_{\mathrm{S}}$ if each of the individual uncertainties is known. However, $A, \beta, W_{\mathrm{e}}, W_{1}$ and $\rho_{\text {nact }}$ are calculated quantities, so their uncertainties were not known, but had to be calculated using equation A.3. 
The flow in the air duct was measured using a venturi tube measurement. The nozzle throat area $A_{\mathrm{n}}$, which is part of equation A.16, was calculated from the throat diameter. Thus its uncertainty can be evaluated very easily.

$$
\begin{array}{r}
A_{\mathrm{n}}=\frac{\pi d_{\mathrm{n}}^{2}}{4} \\
E_{A_{\mathrm{n}}}=\frac{\partial A_{\mathrm{n}}}{\partial d_{\mathrm{n}}} \mathrm{d} d_{\mathrm{n}}=\frac{\pi d_{\mathrm{n}}}{2} \mathrm{~d} d_{\mathrm{n}}
\end{array}
$$

The uncertainty of the throat diameter was given to be: $E_{d n}=d d d_{n}= \pm 0.254 \mathrm{~mm}= \pm 0.01 \mathrm{in}$.

The area ratio factor $\beta$ was calculated using the equation:

$$
\beta=\frac{A_{\mathrm{n}}}{A_{\mathrm{en}}}=\frac{d_{\mathrm{n}}^{2}}{d_{\mathrm{en}}^{2}}
$$

Again, both areas were calculated using their diameter given by the manufacturer of the venturi tube. The partial derivatives used to evaluate the uncertainty in $\beta$ were:

$$
\begin{aligned}
& \frac{\partial \beta}{\partial d_{\mathrm{n}}}=2 \frac{d_{\mathrm{n}}}{d_{\mathrm{en}}{ }^{2}} \\
& \frac{\partial \beta}{\partial d_{\mathrm{en}}}=-2 \frac{d_{\mathrm{n}}{ }^{2}}{d_{\mathrm{en}}{ }^{3}}
\end{aligned}
$$

Using these derivatives to rewrite A.3 gives:

$$
E_{\beta}=\left[\left(2\left(d_{\mathrm{n}} / d_{\mathrm{en}}^{2}\right) \mathrm{d} d_{\mathrm{n}}\right)^{2}+\left(-2\left(d_{\mathrm{n}}^{2} / d_{\mathrm{en}}^{3}\right) \mathrm{d} d_{\mathrm{en}}\right)^{2}\right]^{1 / 2}
$$

The required uncertainty in the inlet diameter was also $\mathrm{E}_{\mathrm{d}_{\mathrm{en}}}=\mathrm{dd}_{\mathrm{en}}= \pm 0.254 \mathrm{~mm}= \pm 0.01 \mathrm{in}$.

The humidity ratios $W_{\mathrm{e}}$ and $W_{\mathrm{l}}$ are a function of the water vapor pressure $p_{\mathrm{w}}$ and the atmospheric pressure $p$.

$$
W=0.62198 \frac{p_{\mathrm{w}}}{p-p_{\mathrm{w}}}
$$

The factor 0.62198 comes from the ratio of the mole weights of the two components, water and air. 
The required partial derivatives of equation A.31 are:

$$
\begin{aligned}
& \frac{\partial W}{\partial p}=0.62198 \frac{p_{\mathrm{w}}}{\left(p-p_{\mathrm{w}}\right)^{2}} \\
& \frac{\partial W}{\partial p_{\mathrm{w}}}=0.62198 \frac{p}{\left(p-p_{\mathrm{w}}\right)^{2}}
\end{aligned}
$$

They lead to the uncertainty in $W$ :

$$
E_{W}=d W=\left[\left(\frac{\partial W}{\partial p} \mathrm{~d} p\right)^{2}+\left(\frac{\partial W}{\partial p_{w}} \mathrm{~d} p_{\mathrm{w}}\right)^{2}\right]^{1 / 2}
$$

Unfortunately the water saturation pressure is a calculated quantity itself, which means its uncertainty had to be calculated.

The equation that was used to calculate the saturation pressure from the dew-point temperature, $T_{\text {dew }}$, ( $\left.{ }^{\circ} \mathrm{R}\right)$, is given below. The equation was assumed to cause no additional uncertainties.

$$
p_{\mathrm{w}}=\operatorname{EXP}\left[\frac{C_{8}}{T_{\text {dew }}}+C_{9}+C_{10} T_{\text {dew }}+C_{11} T_{\text {dew }}{ }^{2}+C_{12} T_{\text {dew }}{ }^{3}+C_{13} \ln T_{\text {dew }}\right]
$$

The partial derivative of equation A.35 with respect to $T_{\text {dew }}$ is:

$$
\frac{\partial p_{\mathrm{w}}}{\partial T_{\text {dew }}}=\left[\frac{-C_{8}}{T_{\text {dew }}{ }^{2}}+C_{10}+2 C_{11} T_{\text {dew }}+3 C_{12} T_{\text {dew }}^{2}+\frac{C_{13}}{T_{\text {dew }}}\right] p_{\mathrm{w}}
$$

The uncertainty in $p_{\mathrm{w}}$ is now given by:

$$
E_{p_{\mathrm{w}}}=\mathrm{d} p_{\mathrm{w}}=\frac{\partial p_{\mathrm{w}}}{\partial T_{\mathrm{dew}}} \mathrm{d} T_{\mathrm{dew}}
$$

As already mentioned in section A.3, the uncertainty of the dew-point temperature measurement was given to be: $\mathrm{E}_{\mathrm{T}_{\text {dew }}}=\mathrm{dT}_{\mathrm{dew}}= \pm 0.05 \%$ of reading .

Finally, the uncertainty in the moist air's density $\rho_{\text {nact }}$ had to be evaluated. The density was calculated using the ideal gas equation and the humidity ratio.

$$
\rho_{\text {nact }}=\frac{p_{\mathrm{n}} 144(1+W)}{R_{\mathrm{a}} T_{\mathrm{n}}(1+1.6078 W)}
$$

The factor 1.6078 is the ratio of the molar weights of air and water. 
The partial derivatives of the A.38 are:

$$
\begin{aligned}
\frac{\partial \rho_{\text {nact }}}{\partial p_{\mathrm{n}}}= & \frac{144(1+W)}{R T_{\mathrm{n}}(1+1.6078 W)} \\
\frac{\partial \rho_{\text {nact }}}{\partial T_{\mathrm{n}}} & =\frac{-p_{\mathrm{n}} 144(1+W)}{R T_{\mathrm{n}}^{2}(1+1.6078 W)} \\
\frac{\partial \rho_{\text {nact }}}{\partial W} & =\frac{-0.6078 p_{\mathrm{n}} 144}{R T_{\mathrm{n}}(1+1.6078 W)^{2}}
\end{aligned}
$$

Rewriting equation A.3 with the above partial derivatives gives:

$$
E_{\rho_{\text {nact }}}=\left[\left(\frac{\partial \rho_{\text {nact }}}{\partial p_{\mathrm{n}}} \mathrm{d} p_{\mathrm{n}}\right)^{2}+\left(\frac{\partial \rho_{\text {nact }}}{\partial T_{\mathrm{n}}} \mathrm{d} T_{\mathrm{n}}\right)^{2}+\left(\frac{\partial \rho_{\text {nact }}}{\partial W} \mathrm{~d} W\right)^{2}\right]
$$

The pressure $p_{\mathrm{n}}$ in the nozzle throat was calculated as the difference of atmospheric pressure and nozzle pressure drop. The uncertainty of the nozzle pressure can be derived as follows:

$$
\begin{gathered}
p_{\mathrm{n}}=p_{\mathrm{atm}}-\Delta p \\
E_{p_{\mathrm{n}}}=\mathrm{d} p_{\mathrm{n}}=\left[\left(\mathrm{d} p_{\mathrm{atm}}\right)^{2}+(\mathrm{d} \Delta p)^{2}\right]^{1 / 2}
\end{gathered}
$$

The uncertainties of the pressure measurements were given from manufacturer data: $\mathrm{E}_{\mathrm{p}_{\mathrm{atm}}}=\mathrm{dp}_{\mathrm{atm}}= \pm 0.3429 \mathrm{mmHg}= \pm 0.0135 \mathrm{in} \mathrm{Hg}$ $E_{D_{n}}=d D p_{n}= \pm 2.489 \mathrm{mmH}_{2} \mathrm{O}= \pm 0.098$ in $\mathrm{H}_{2} \mathrm{O}$.

\section{A. 5. 2 Uncertainty of the Latent Capacity}

The latent cooling capacity (ASHRAE Standard 116-1983) is given by:

$$
\dot{Q}_{\mathrm{L}}=6360060 C_{\mathrm{D}} A_{\mathrm{n}}\left(W_{\mathrm{e}}-W_{\mathrm{l}}\right)\left[\frac{2 g_{\mathrm{C}} \Delta p_{\mathrm{n}} \rho_{\text {nact }}}{144\left(1-\beta^{2}\right)}\right]^{1 / 2}
$$

where:

$$
\begin{array}{lll}
C_{\mathrm{D}} & = & \text { nozzle discharge coefficient }(0.986) \\
A_{\mathrm{n}} & = & \text { nozzle throat area }\left(\mathrm{ft}^{2}\right) \\
W_{\mathrm{e}} & = & \text { entering humidity ratio }\left(\mathrm{lb} \mathrm{H}_{2} \mathrm{O} / \mathrm{lb} \text { dry air }\right) \\
W_{\mathrm{l}} & = & \text { leaving humidity ratio }\left(\mathrm{lb} \mathrm{H}_{2} O / \mathrm{lb} \text { dry air }\right) \\
g_{\mathrm{C}} & = & \text { gravity constant }\left(32.174 \mathrm{ft} \cdot \mathrm{lb}_{\mathrm{m}} / \mathrm{lb}_{\mathrm{f}} \cdot \mathrm{s}^{2}\right) \\
\Delta p_{\mathrm{n}} & = & \text { static pressure drop across nozzle }(\mathrm{psia})
\end{array}
$$




$$
\begin{array}{lll}
\rho_{\text {nact }} & =\text { density of the moist air }\left(\mathrm{lb} / \mathrm{ft}^{3}\right) \\
144 & = & \text { unit conversion factor from in }{ }^{2} \text { to } \mathrm{ft}^{2} \\
\beta & = & \text { area relation factor }(0.250723)
\end{array}
$$

The partial derivatives of this equation are:

$$
\begin{gathered}
\frac{\partial \dot{Q}_{\mathrm{L}}}{\partial A_{\mathrm{n}}}=6360060 C_{\mathrm{D}}\left(W_{\mathrm{e}}-W\right)_{\mathrm{l}}\left[\frac{2 g_{\mathrm{C}} \Delta p_{\mathrm{n}} \rho_{\mathrm{nact}}}{144\left(1-\beta^{2}\right)}\right]^{1 / 2} \\
\frac{\partial \dot{Q}_{\mathrm{L}}}{\partial W_{\mathrm{e}}}=6360060 C_{\mathrm{D}} A_{\mathrm{n}}\left[\frac{2 g_{\mathrm{C}} \Delta p_{\mathrm{n}} \rho_{\mathrm{nact}}}{144\left(1-\beta^{2}\right)}\right]^{1 / 2} \\
\frac{\partial \dot{Q}_{\mathrm{L}}}{\partial W_{\mathrm{l}}}=-6360060 C_{\mathrm{D}} A_{\mathrm{n}}\left[\frac{2 g_{\mathrm{C}} \Delta p_{\mathrm{n}} \rho_{\text {nact }}}{144\left(1-\beta^{2}\right)}\right]^{1 / 2} \\
\frac{\partial \dot{Q}_{\mathrm{L}}}{\partial \Delta p_{\mathrm{n}}}=3180060 C_{\mathrm{D}} A_{\mathrm{n}}\left(W_{\mathrm{e}}-W_{l}\right)\left[\frac{2 g_{\mathrm{C}} \rho_{\mathrm{nact}}}{144\left(1-\beta^{2}\right) \Delta p_{\mathrm{n}}}\right]^{1 / 2} \\
\frac{\partial \dot{Q}_{\mathrm{L}}}{\partial \rho_{\text {nact }}}=3180060 C_{\mathrm{D}} A_{\mathrm{n}}\left(W_{\mathrm{e}}-W\right)_{1}\left[\frac{2 g_{\mathrm{C}} \Delta p_{\mathrm{n}}}{144\left(1-\beta^{2}\right) \rho_{\mathrm{nact}}}\right]^{1 / 2} \\
\frac{\partial \dot{Q}_{\mathrm{L}}}{\partial \beta}=6360060 C_{\mathrm{D}} A_{\mathrm{n}}\left(W_{\mathrm{e}}-W_{\mathrm{l}}\right) \beta\left[\frac{2 g_{\mathrm{C}} \Delta p_{\mathrm{n}} \rho_{\mathrm{nact}}}{144\left(1-\beta^{2}\right)^{3}}\right]^{1 / 2}
\end{gathered}
$$

If the above derivatives are used to rewrite equation A.3, one obtains the uncertainty of the latent capacity:

$$
\begin{aligned}
E_{Q_{\mathrm{L}}}=\left[\left(\frac{\partial \dot{\mathrm{Q}}_{\mathrm{L}}}{\partial A_{\mathrm{n}}} \mathrm{d} A_{\mathrm{n}}\right)^{2}\right. & +\left(\frac{\partial \dot{\mathrm{Q}}_{\mathrm{L}}}{\partial W_{\mathrm{e}}} \mathrm{d} W_{\mathrm{e}}\right)^{2}+\left(\frac{\partial \dot{\mathrm{Q}}_{\mathrm{L}}}{\partial W_{\mathrm{l}}} \mathrm{d} W_{\mathrm{l}}\right)^{2}+ \\
& \left.+\left(\frac{\partial \dot{\mathrm{Q}}_{\mathrm{L}}}{\partial \Delta p_{\mathrm{n}}} \mathrm{d} \Delta p_{\mathrm{n}}\right)^{2}+\left(\frac{\partial \dot{\mathrm{Q}}_{\mathrm{L}}}{\partial \rho_{\text {nact }}} \mathrm{d} \rho_{\text {nact }}\right)^{2}+\left(\frac{\partial \dot{\mathrm{Q}}_{\mathrm{L}}}{\partial \beta} \mathrm{d} \beta\right)^{2}\right]^{1 / 2}
\end{aligned}
$$

In this equation, all the needed uncertainties are known. Either because the quantities are directly measured or their uncertainties have already been calculated in Appendix A.4.1.

The final step was calculating the uncertainty of the air side capacity by using the now known uncertainties of sensible and latent capacity in equation A.15. 


\section{APPENDIX C: COOLING MEASUREMENT SUMMARY SHEETS}

\section{Coil 1}

R22, A01102, No Fan

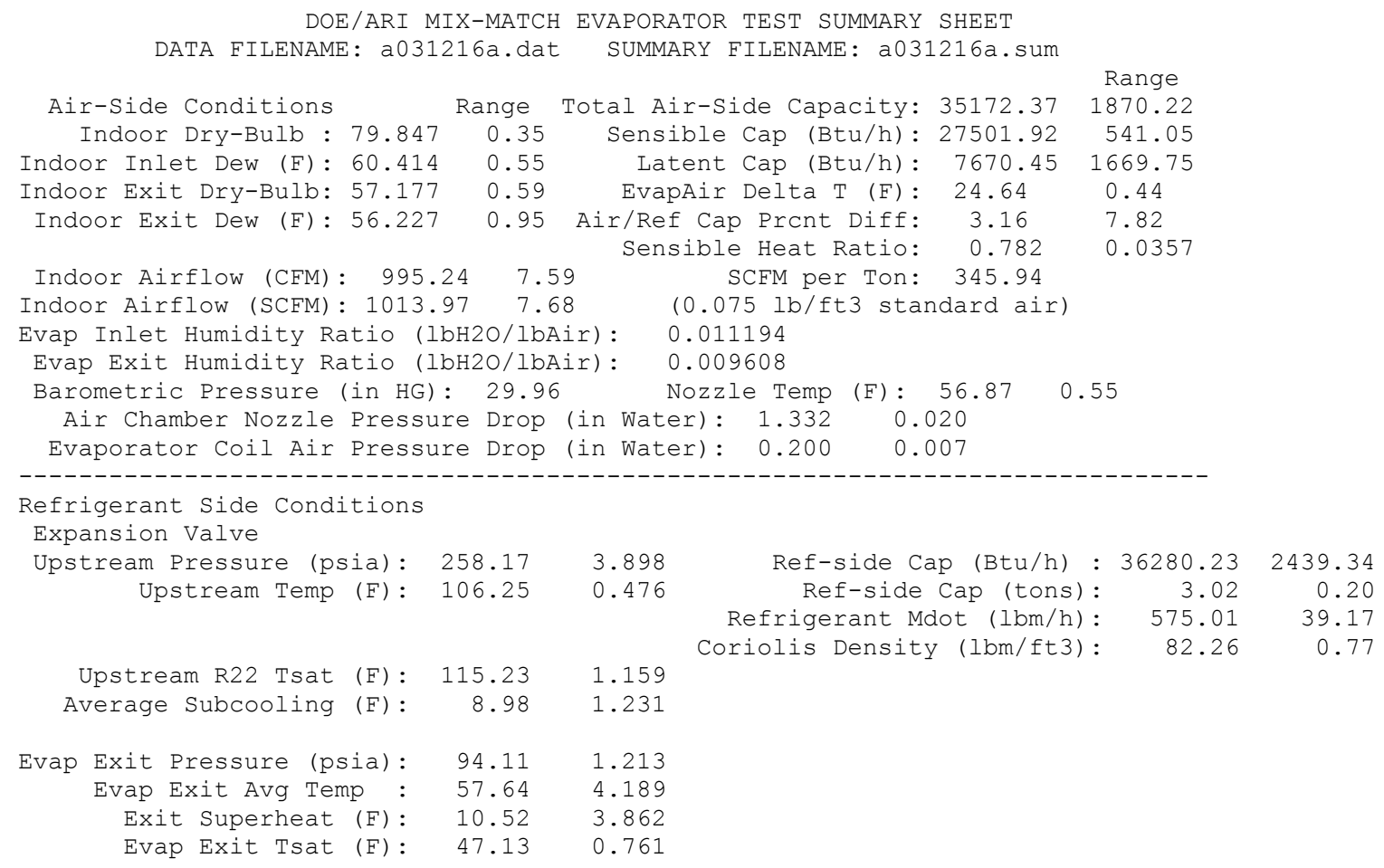


DOE/ARI MIX-MATCH EVAPORATOR TEST SUMMARY SHEET DATA FILENAME: a031218a.dat SUMMARY FILENAME: a031218a.sum

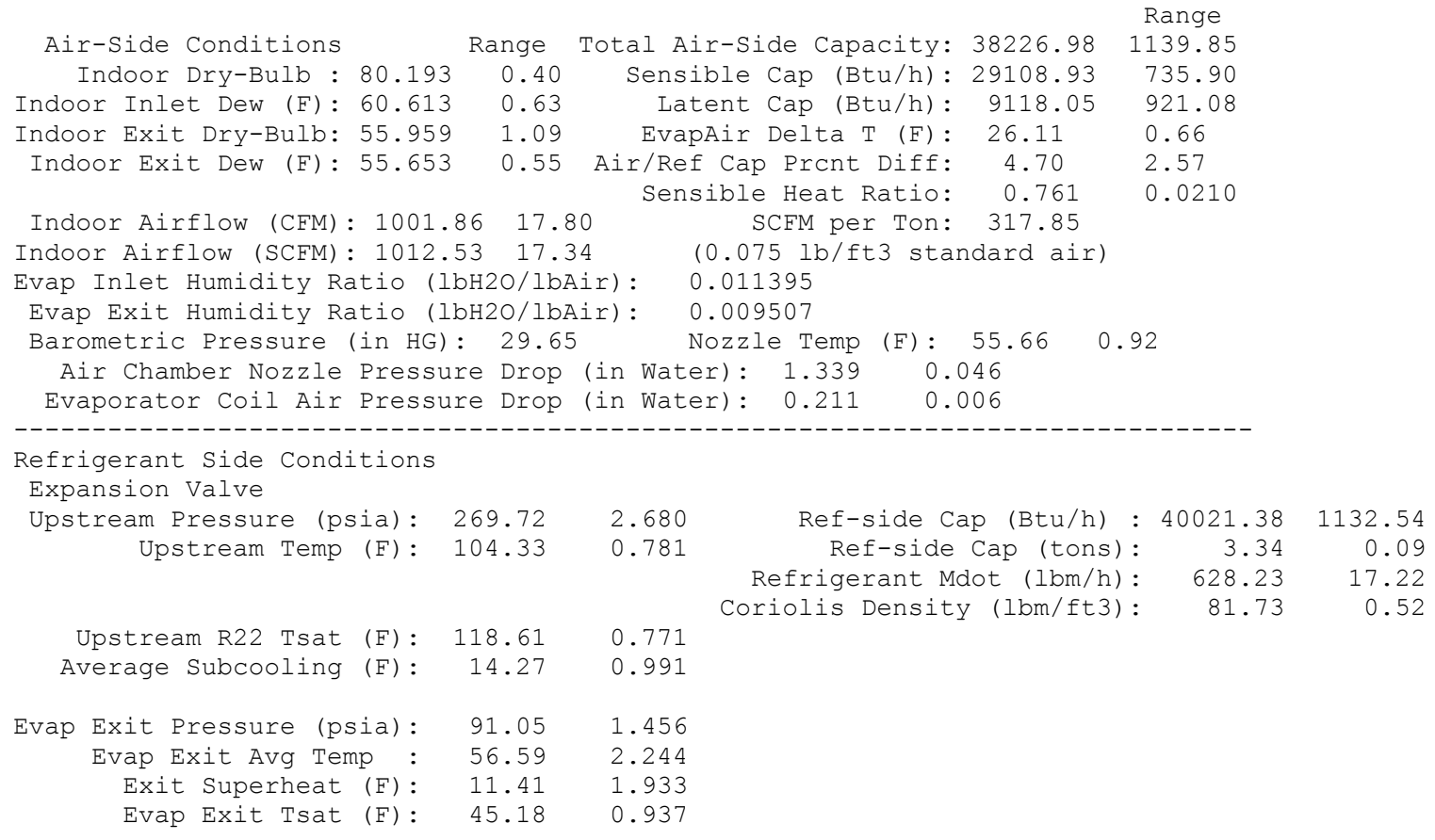


DOE/ARI MIX-MATCH EVAPORATOR TEST SUMMARY SHEET DATA FILENAME: a031218b.dat SUMMARY FILENAME: a031218b.sum

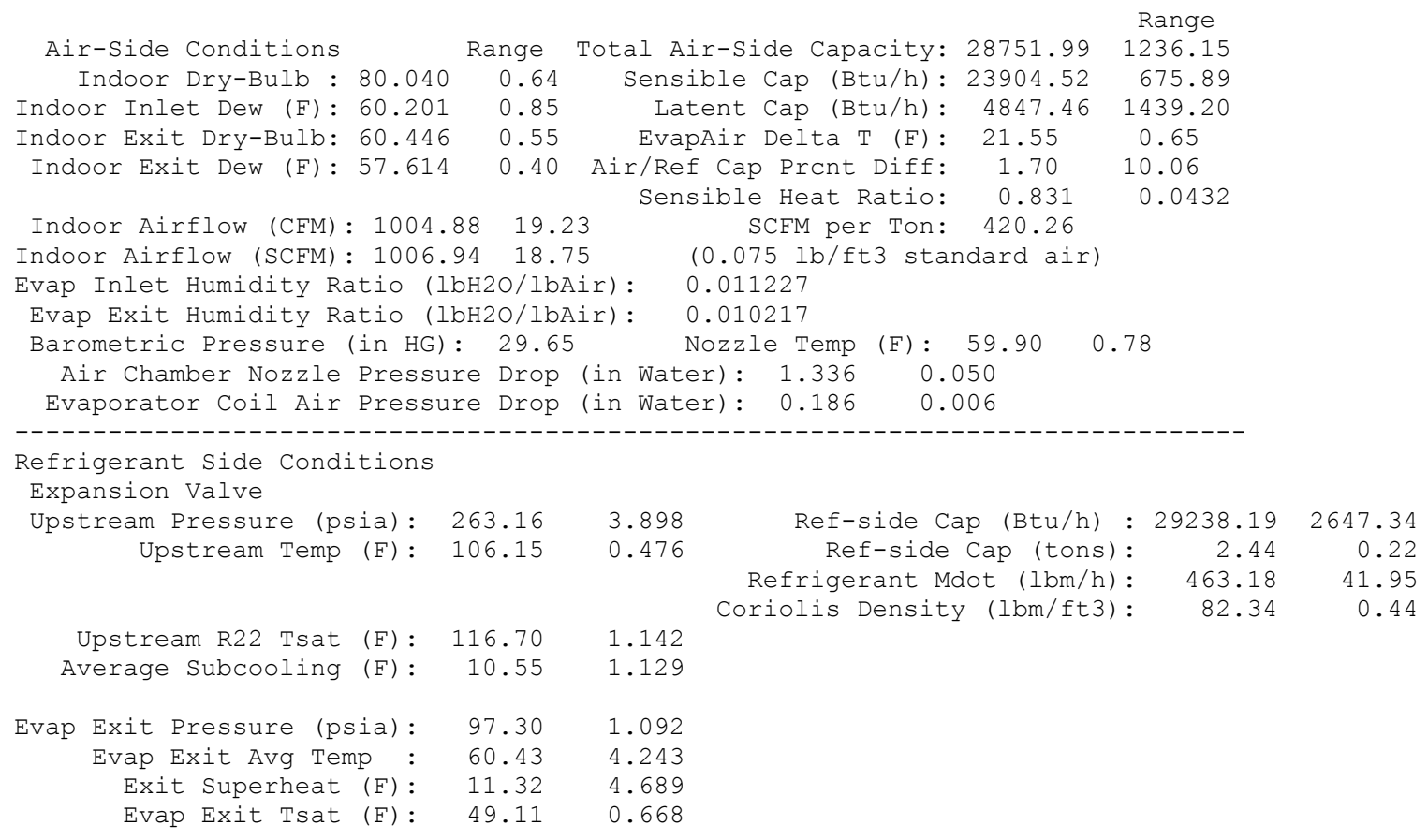


DOE/ARI MIX-MATCH EVAPORATOR TEST SUMMARY SHEET DATA FILENAME: a040105a.dat SUMMARY FILENAME: a040105a.sum

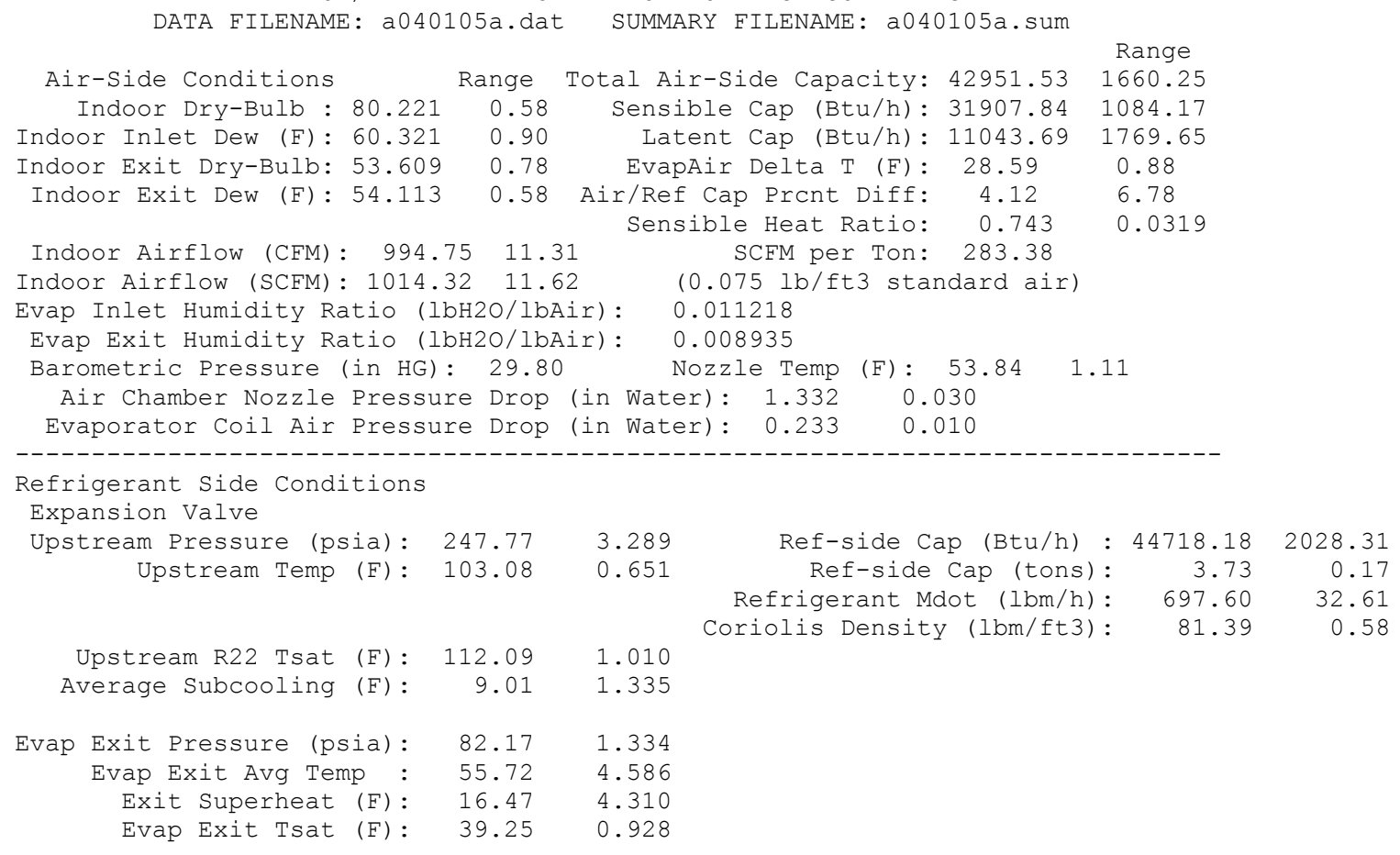


DOE/ARI MIX-MATCH EVAPORATOR TEST SUMMARY SHEET DATA FILENAME: a040106a.dat SUMMARY FILENAME: a040106a.sum

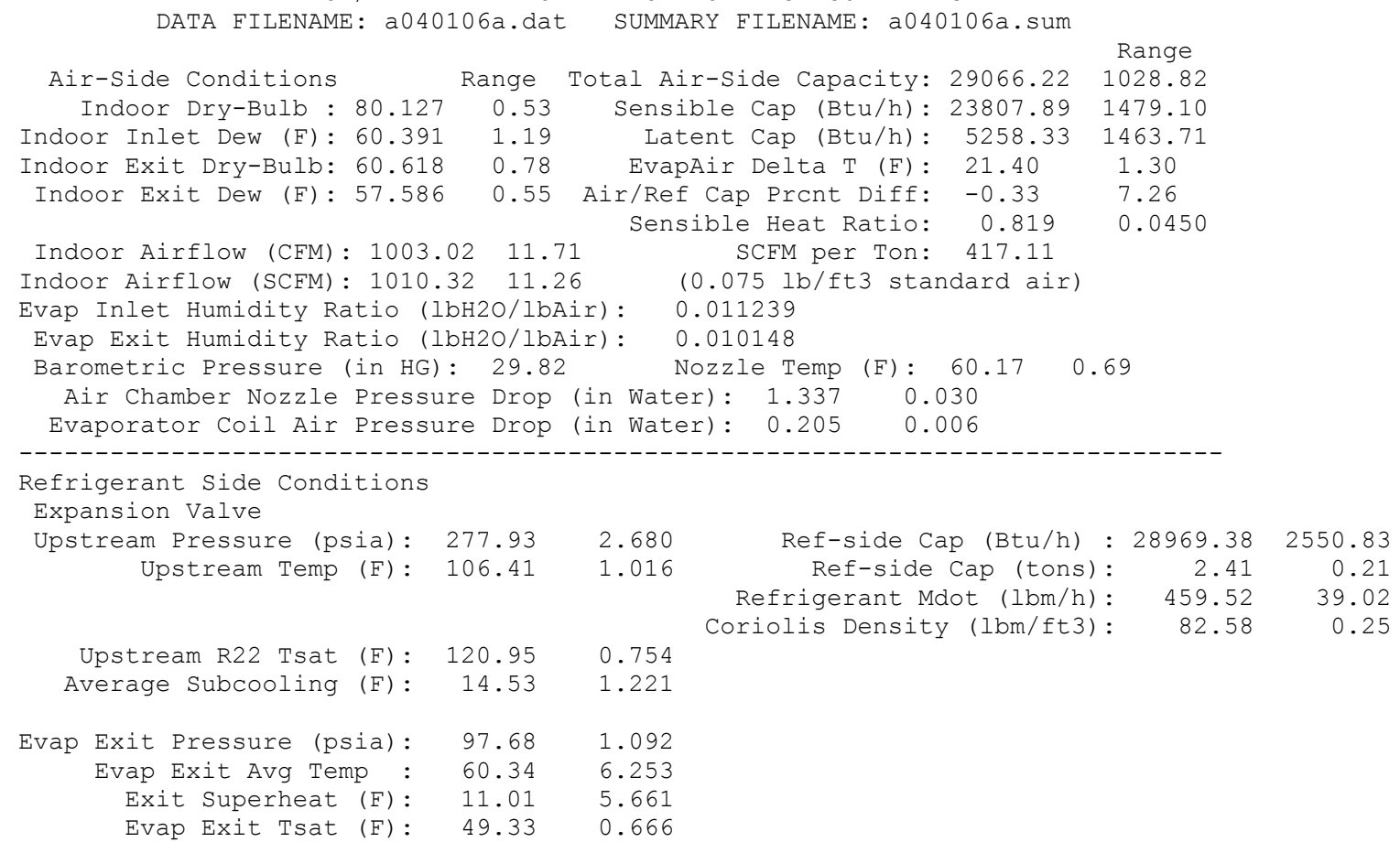


DOE/ARI MIX-MATCH EVAPORATOR TEST SUMMARY SHEET DATA FILENAME: a040106b.dat SUMMARY FILENAME: a040106b.sum

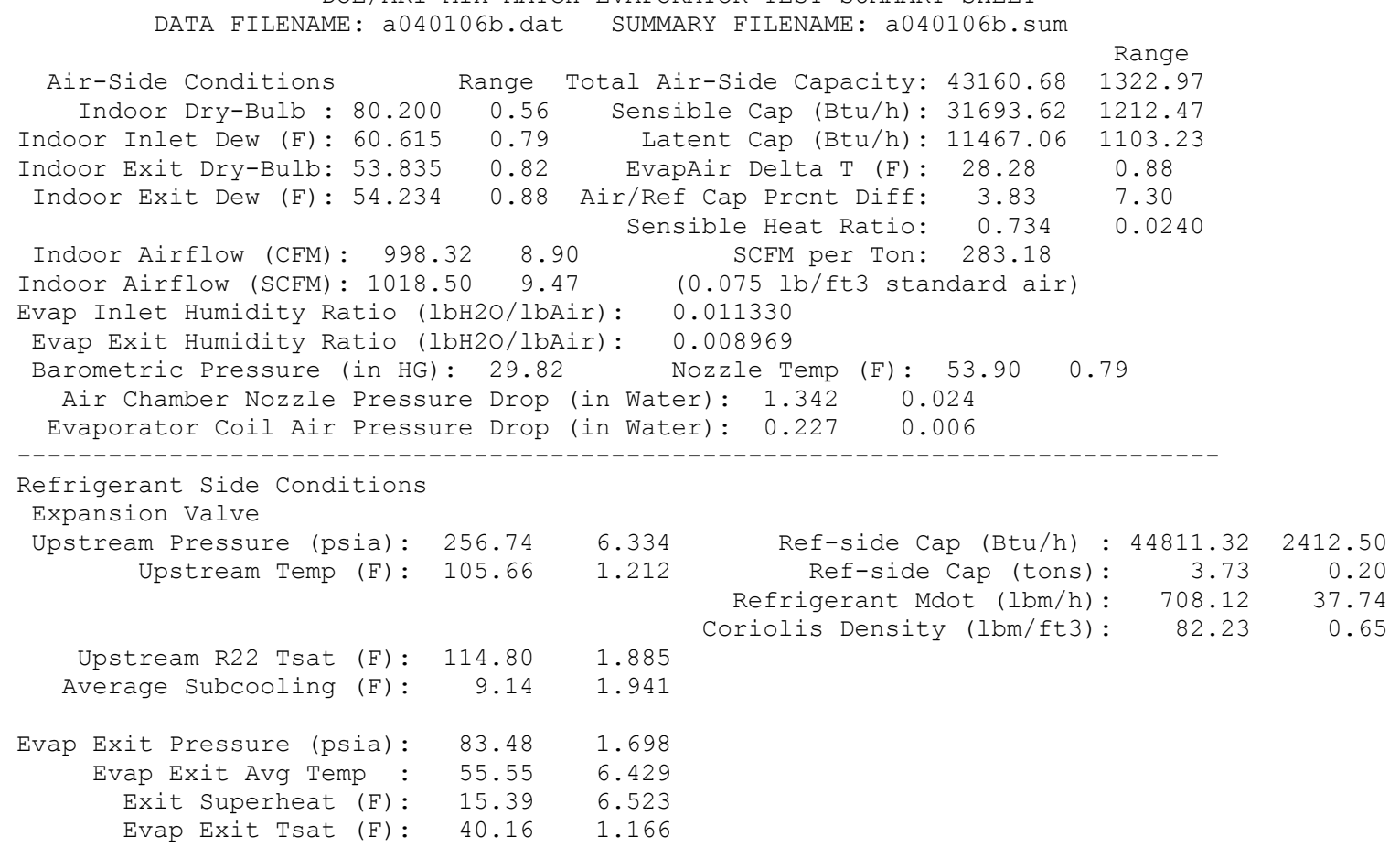




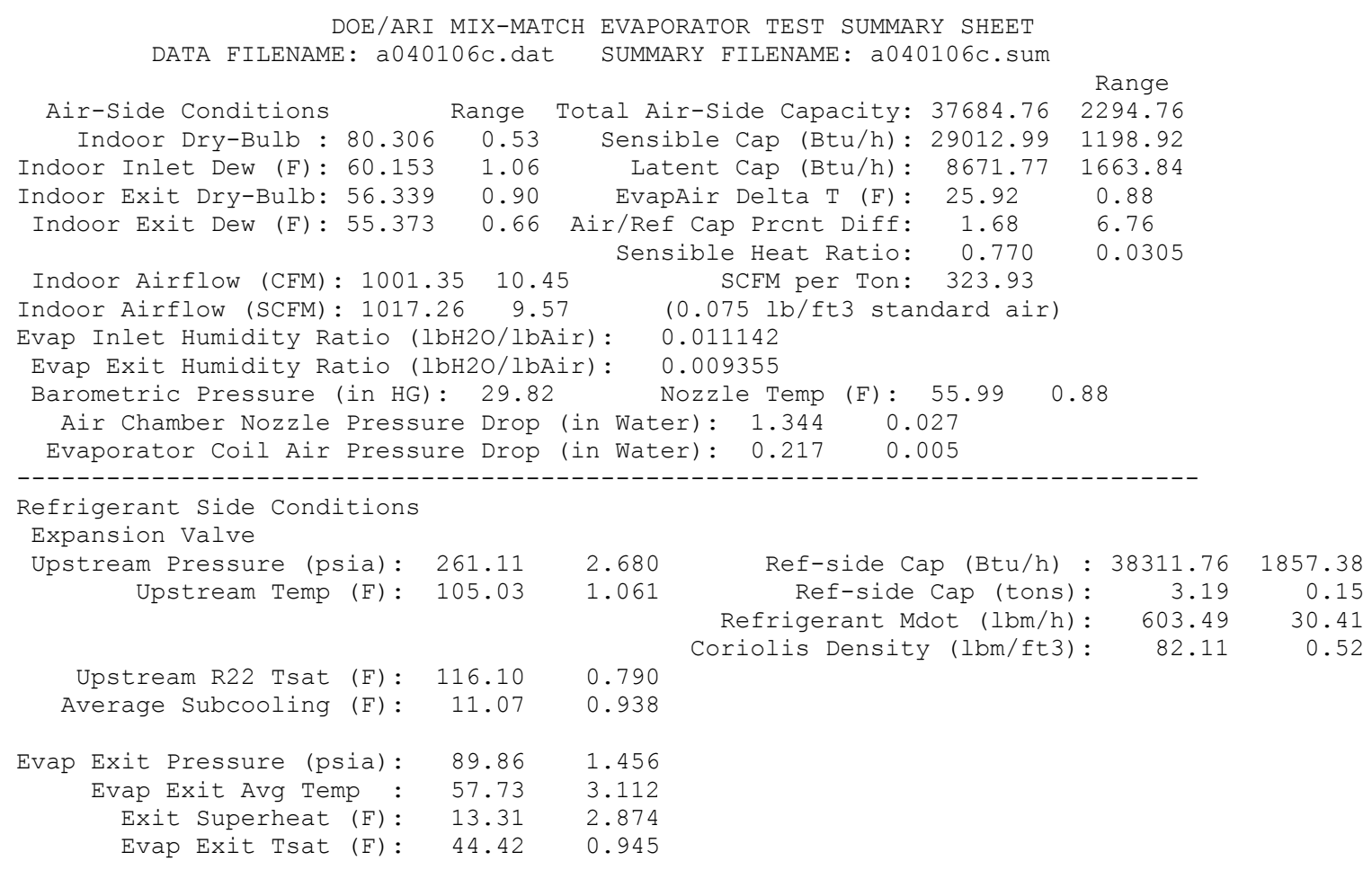




\section{Coil 2}

R22, A01070, No Fan

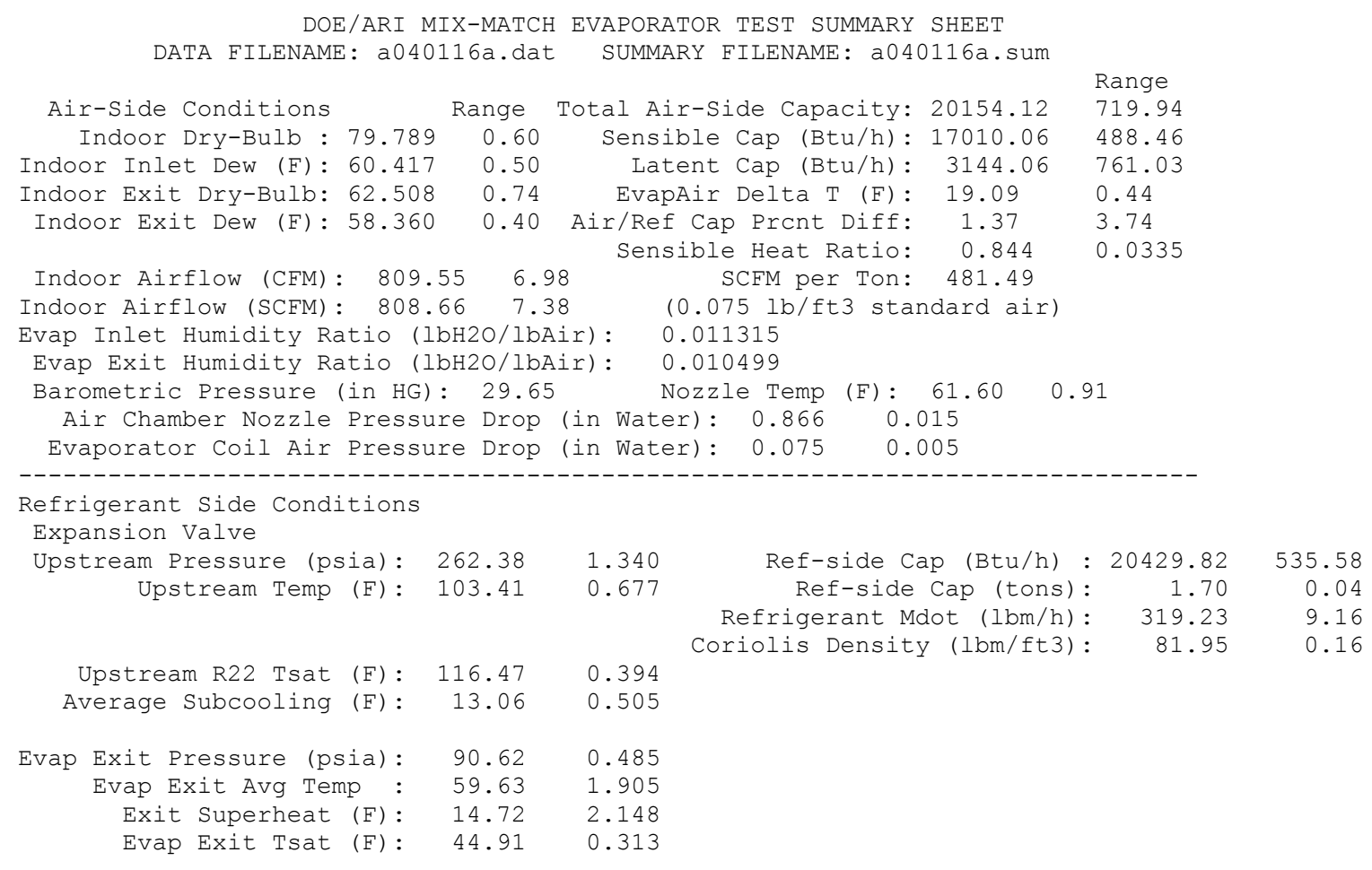


DOE/ARI MIX-MATCH EVAPORATOR TEST SUMMARY SHEET DATA FILENAME: a040116a.dat SUMMARY FILENAME: a040116a.sum

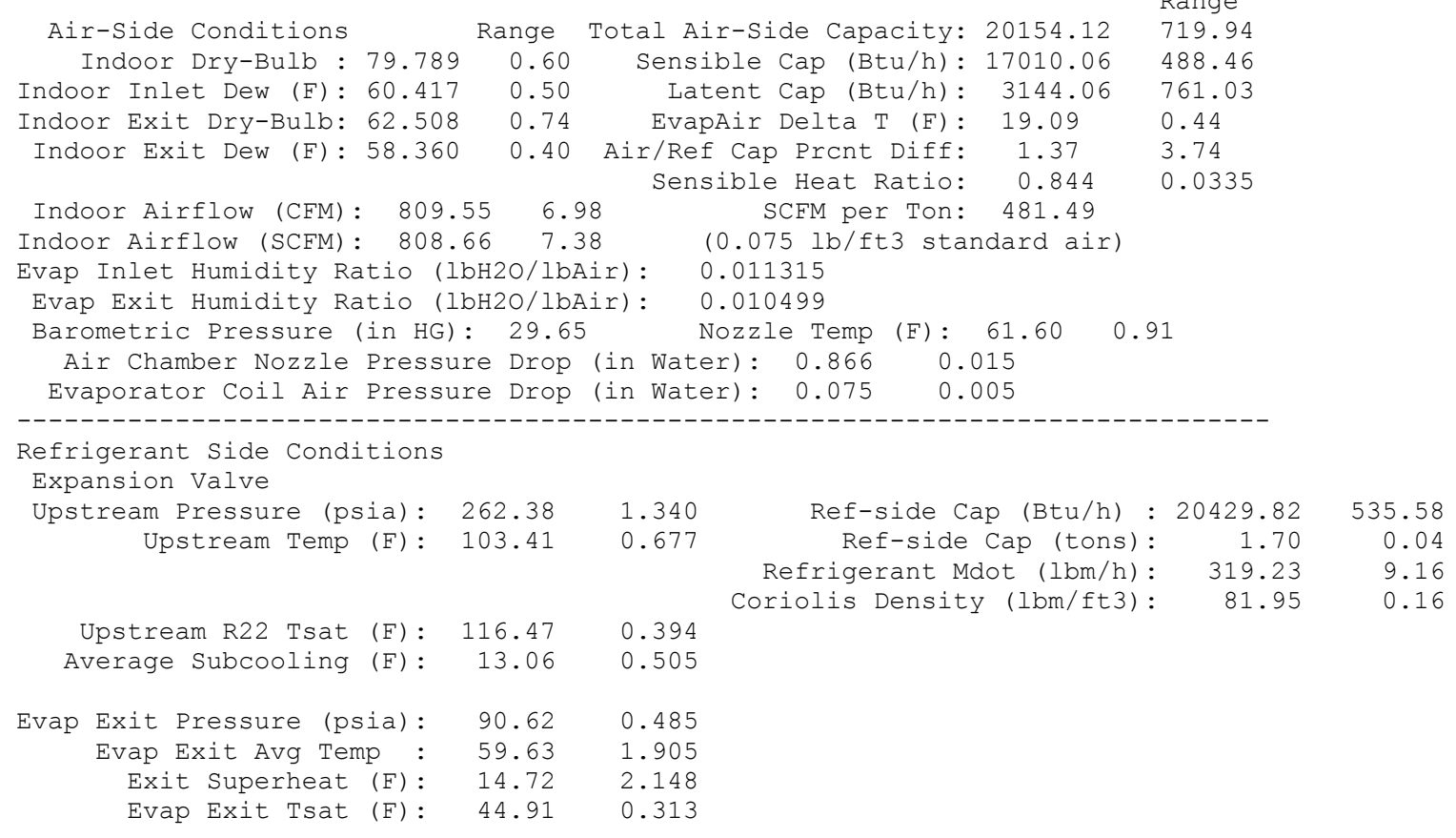


DOE/ARI MIX-MATCH EVAPORATOR TEST SUMMARY SHEET DATA FILENAME: a040116b.dat SUMMARY FILENAME: a040116b.sum

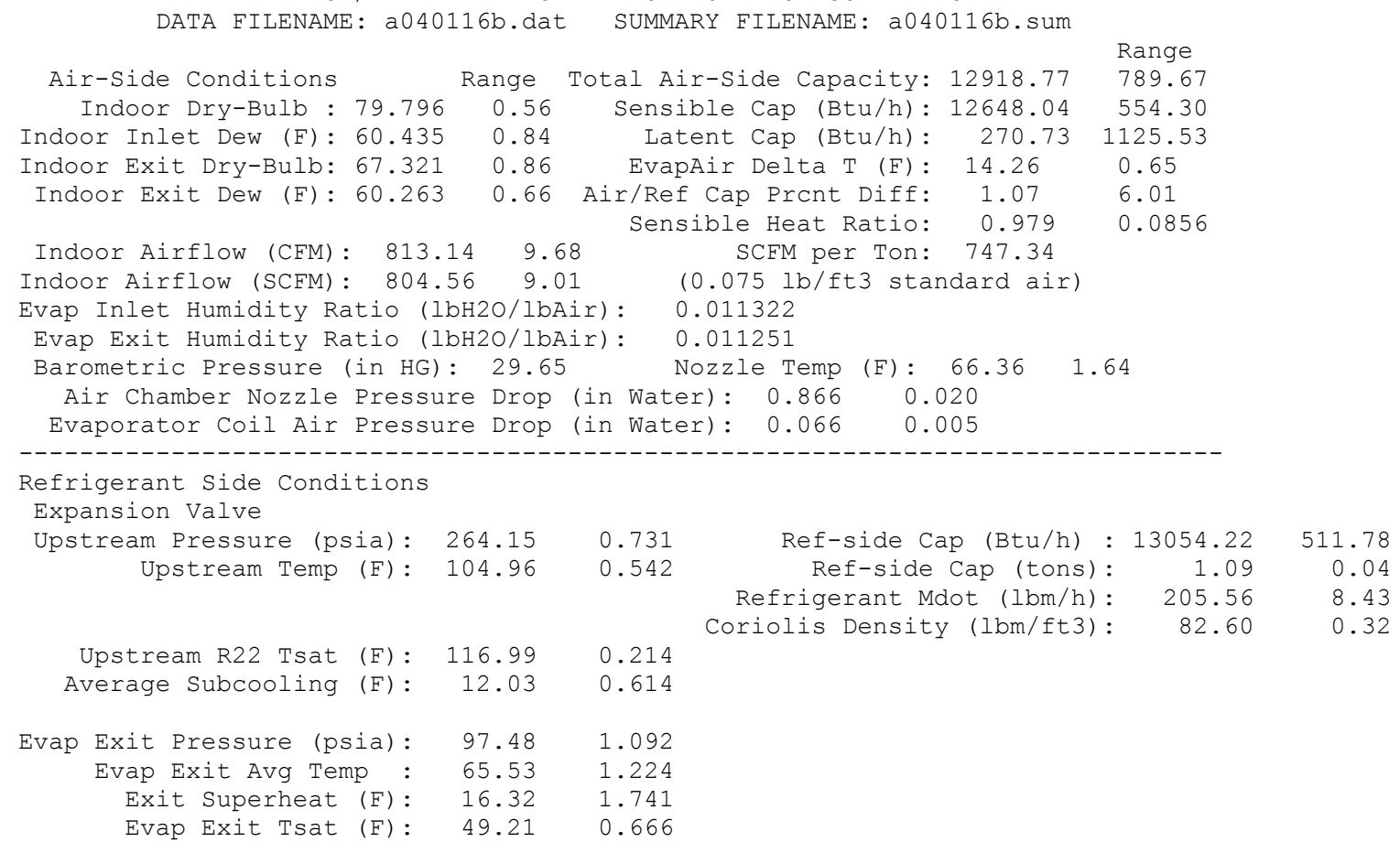


DOE/ARI MIX-MATCH EVAPORATOR TEST SUMMARY SHEET DATA FILENAME: a040119a.dat SUMMARY FILENAME: a040119a.sum

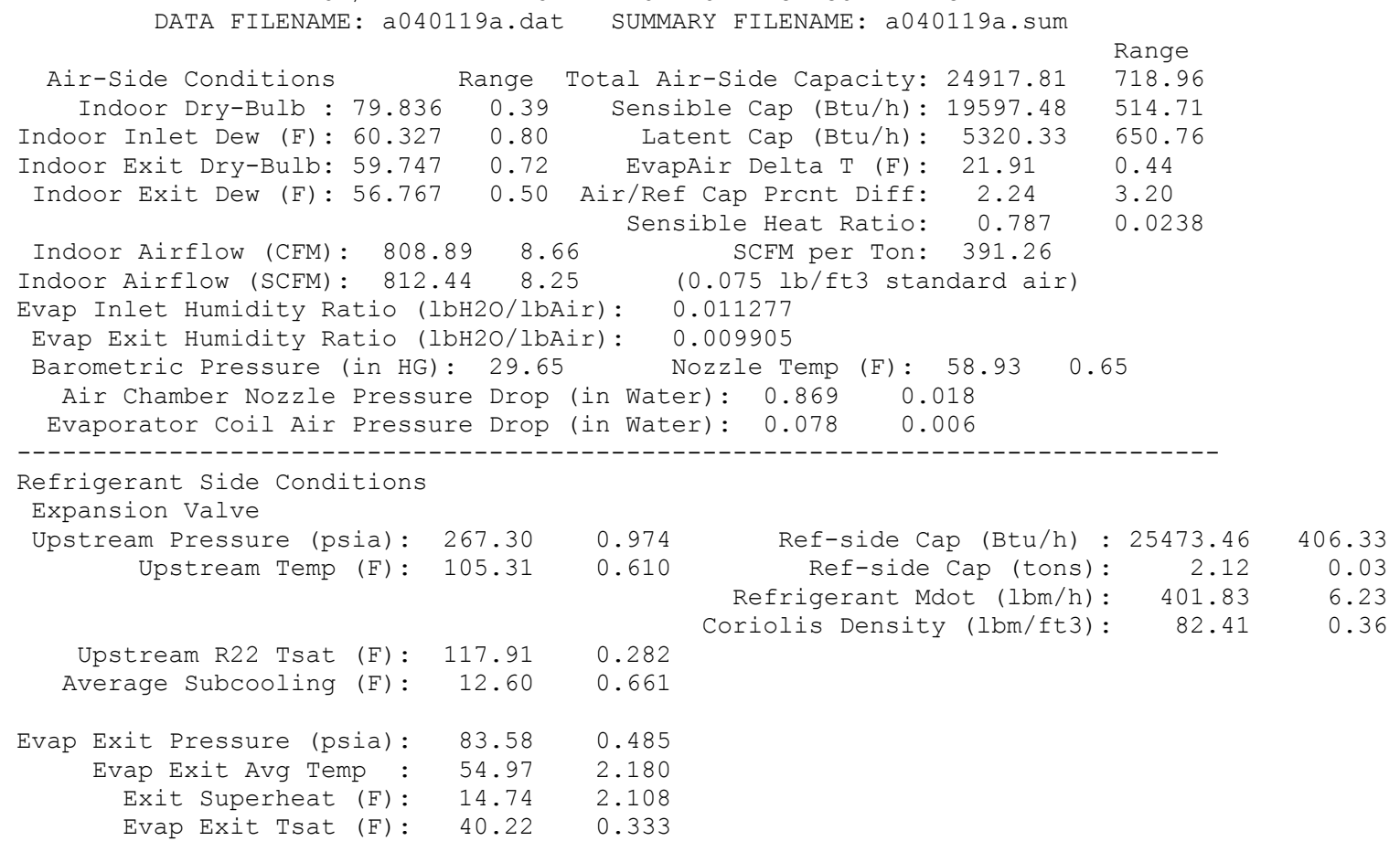


DOE/ARI MIX-MATCH EVAPORATOR TEST SUMMARY SHEET DATA FILENAME: a040119b.dat SUMMARY FILENAME: a040119b.sum

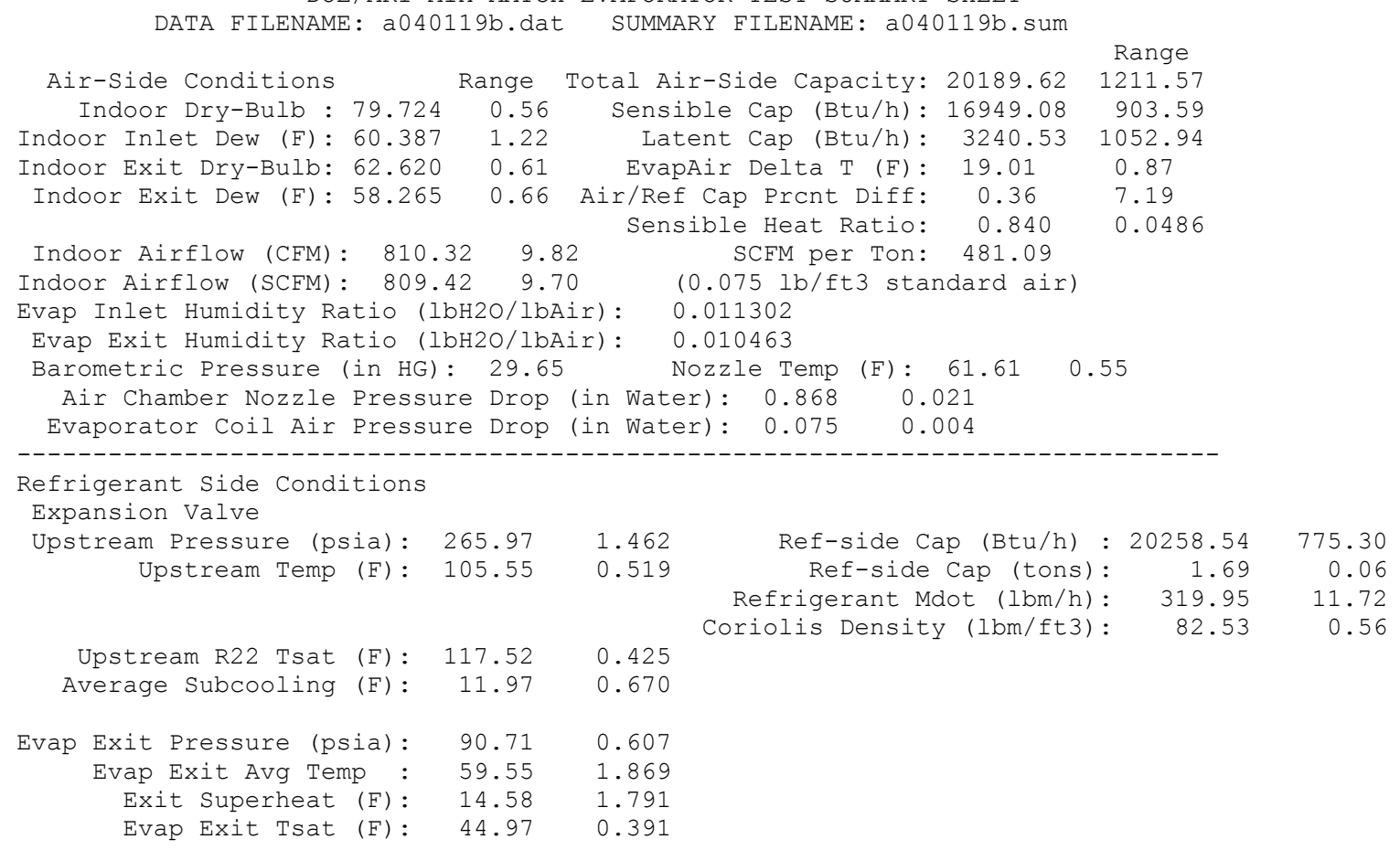


DOE/ARI MIX-MATCH EVAPORATOR TEST SUMMARY SHEET DATA FILENAME: a040121a.dat SUMMARY FILENAME: a040121a.sum

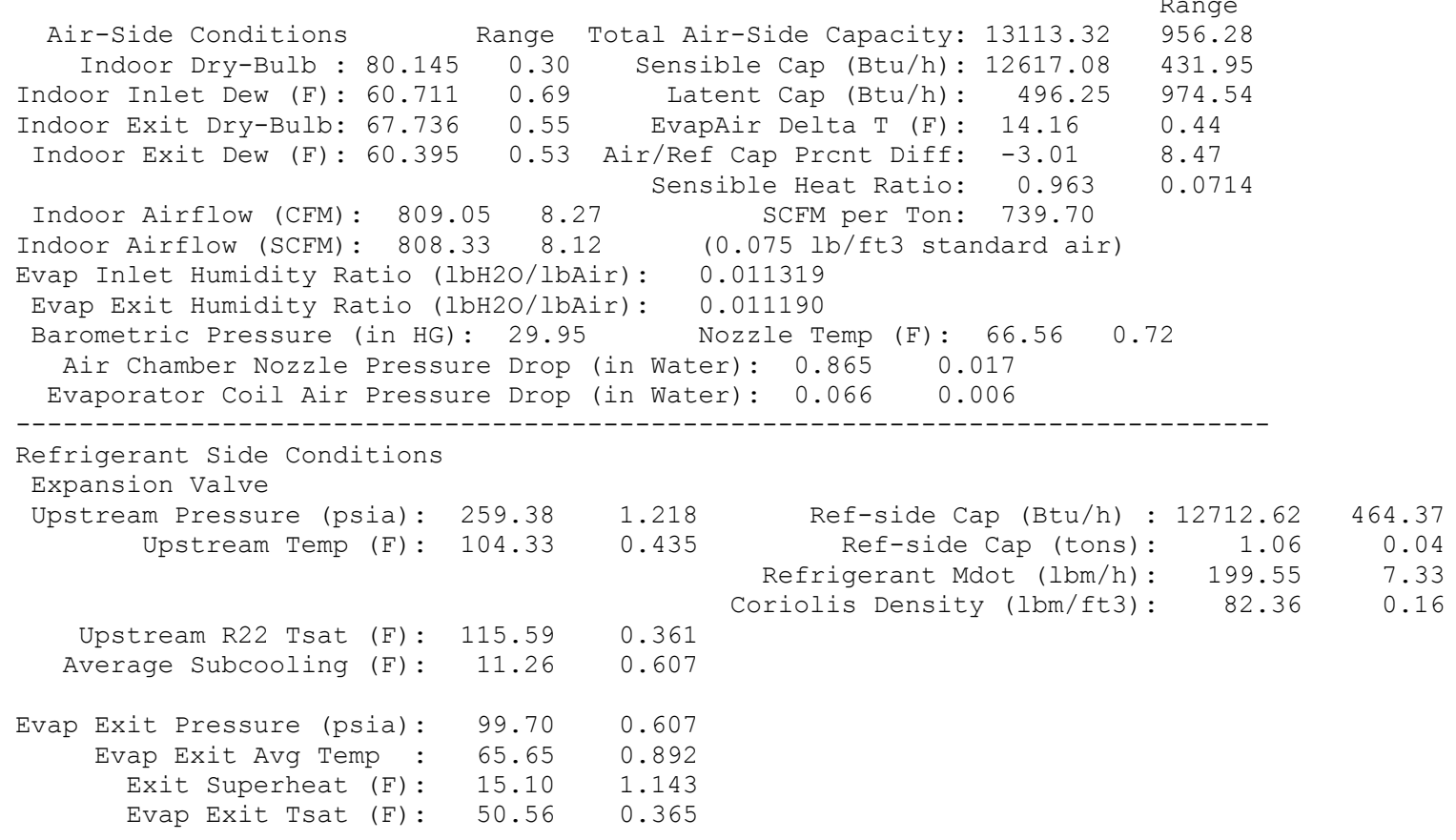




\section{Coil 3}

R22, A01148, No Fan

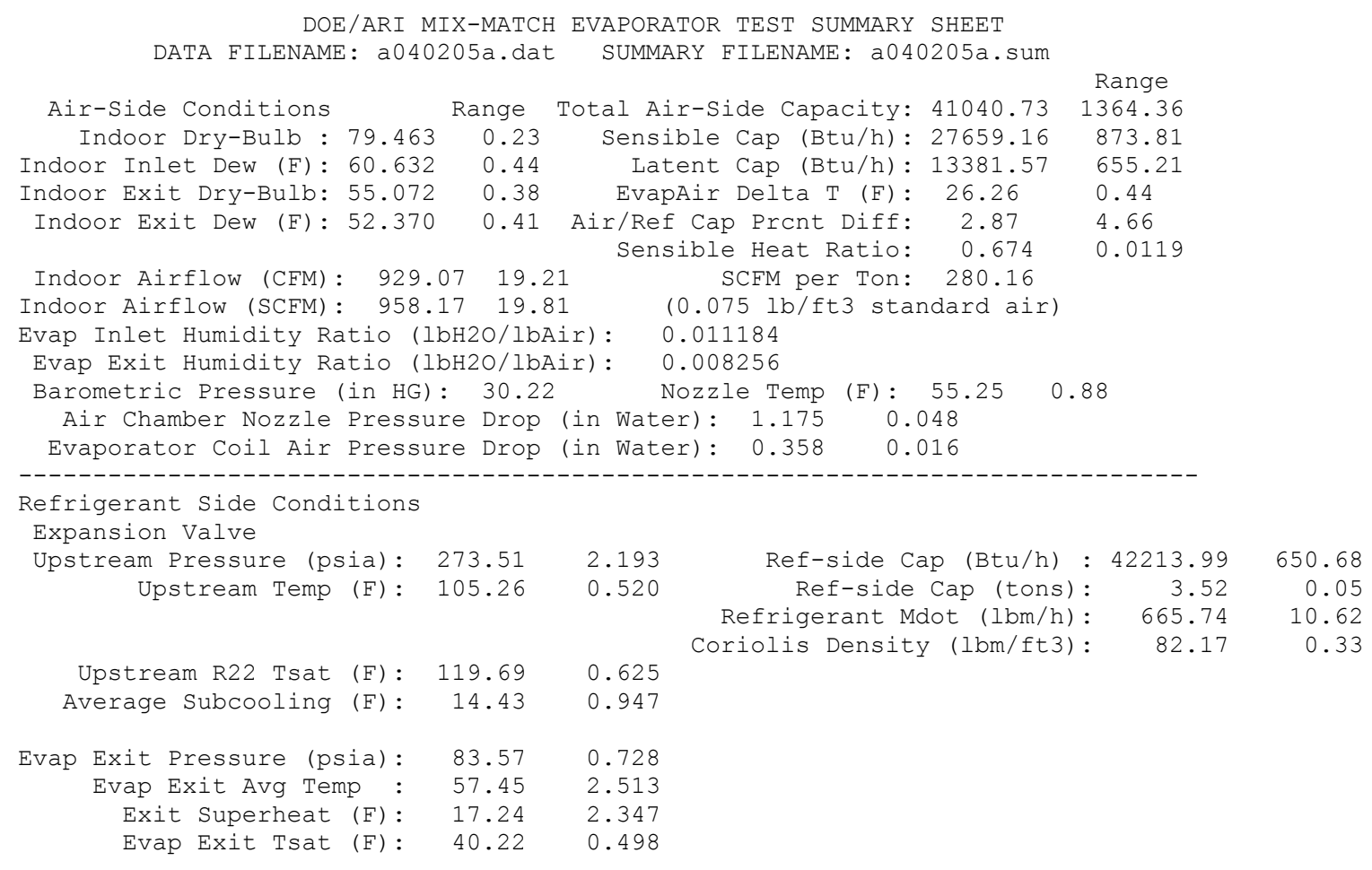


DOE/ARI MIX-MATCH EVAPORATOR TEST SUMMARY SHEET DATA FILENAME: a040209a.dat SUMMARY FILENAME: a040209a.sum

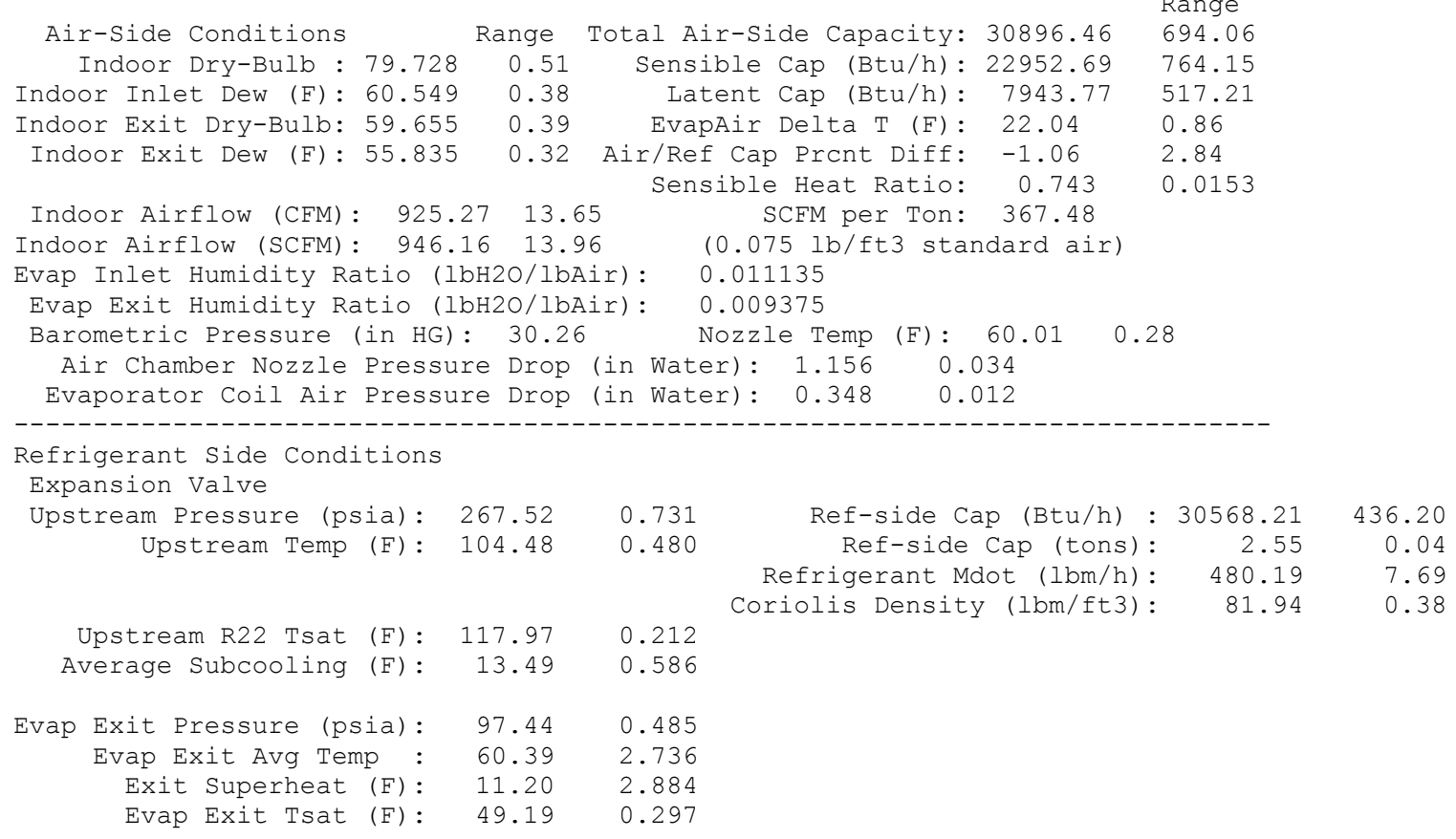


DOE/ARI MIX-MATCH EVAPORATOR TEST SUMMARY SHEET DATA FILENAME: a040209b.dat SUMMARY FILENAME: a040209b.sum

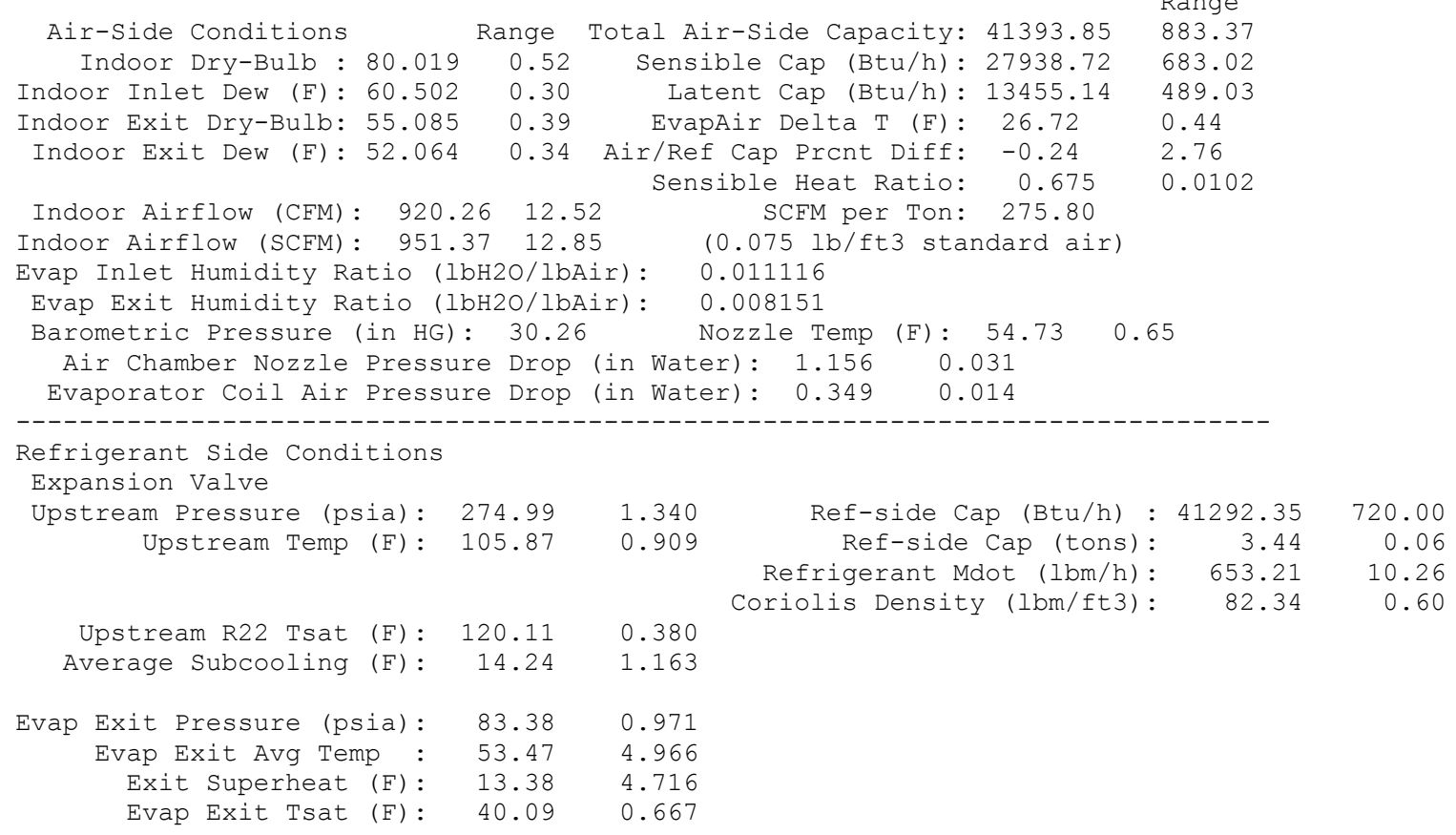


DOE/ARI MIX-MATCH EVAPORATOR TEST SUMMARY SHEET DATA FILENAME: a040210a.dat SUMMARY FILENAME: a040210a.sum

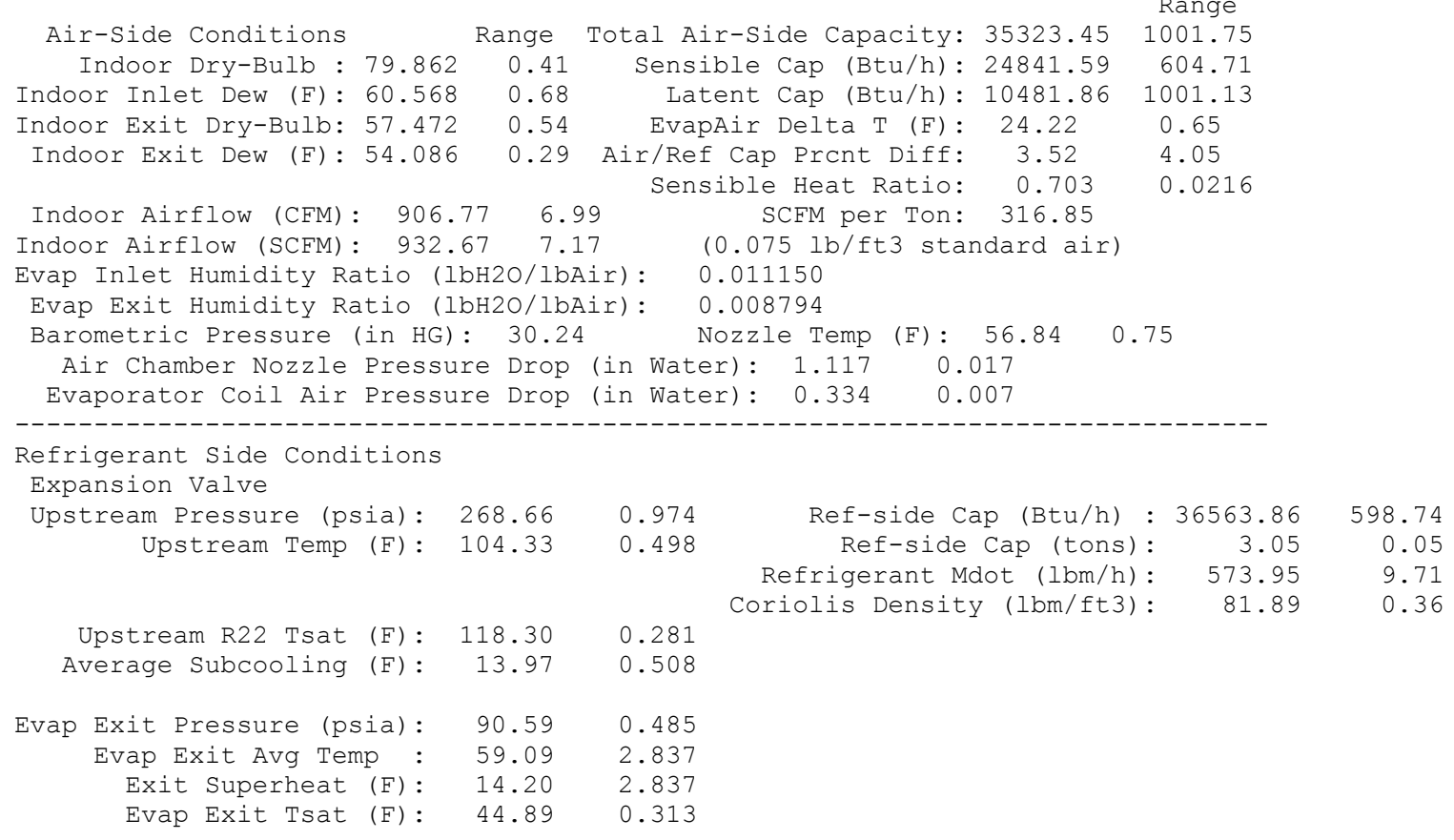


DOE/ARI MIX-MATCH EVAPORATOR TEST SUMMARY SHEET DATA FILENAME: a040211a.dat SUMMARY FILENAME: a040211a.sum

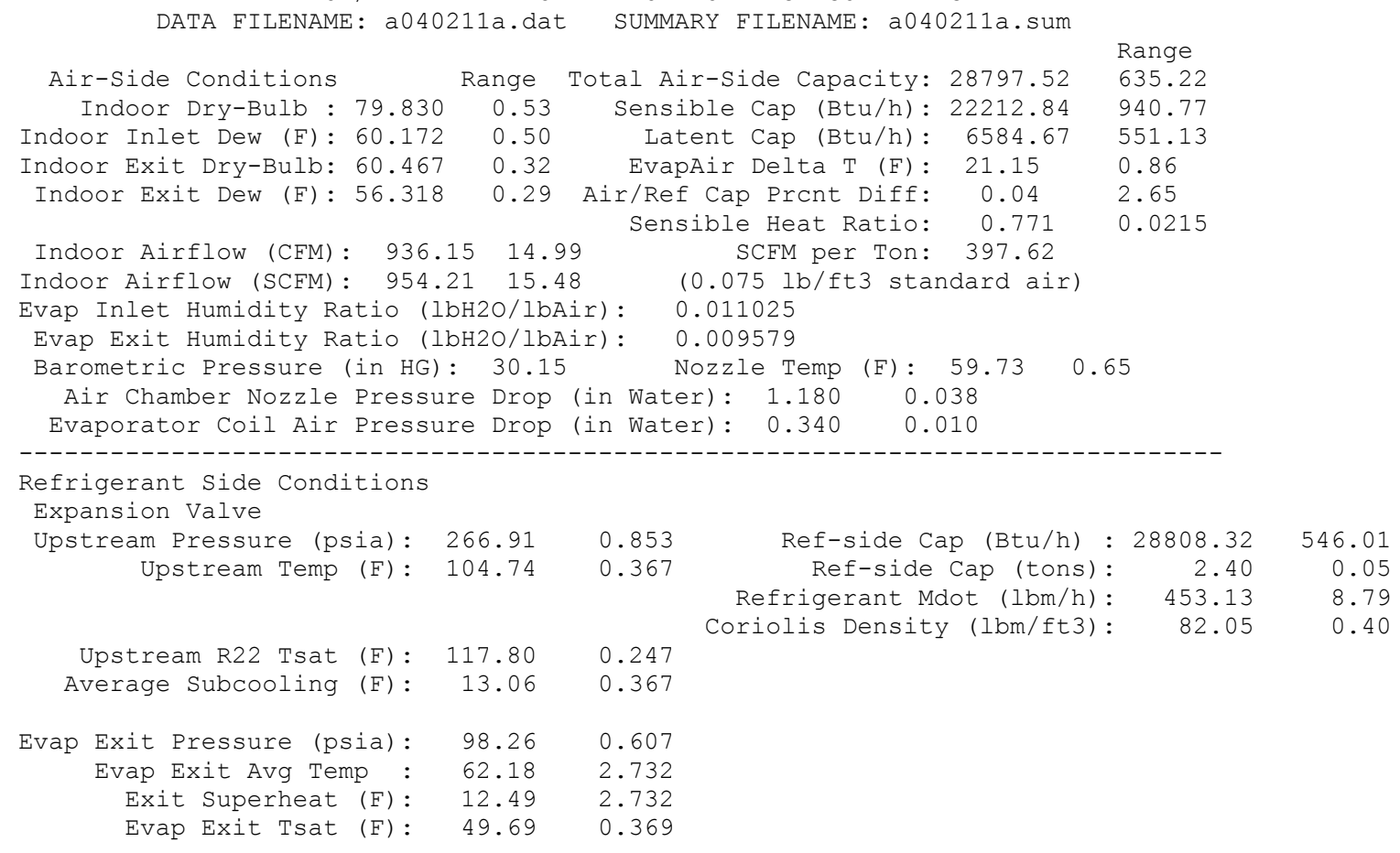


DOE/ARI MIX-MATCH EVAPORATOR TEST SUMMARY SHEET DATA FILENAME: a040211b.dat SUMMARY FILENAME: a040211b.sum

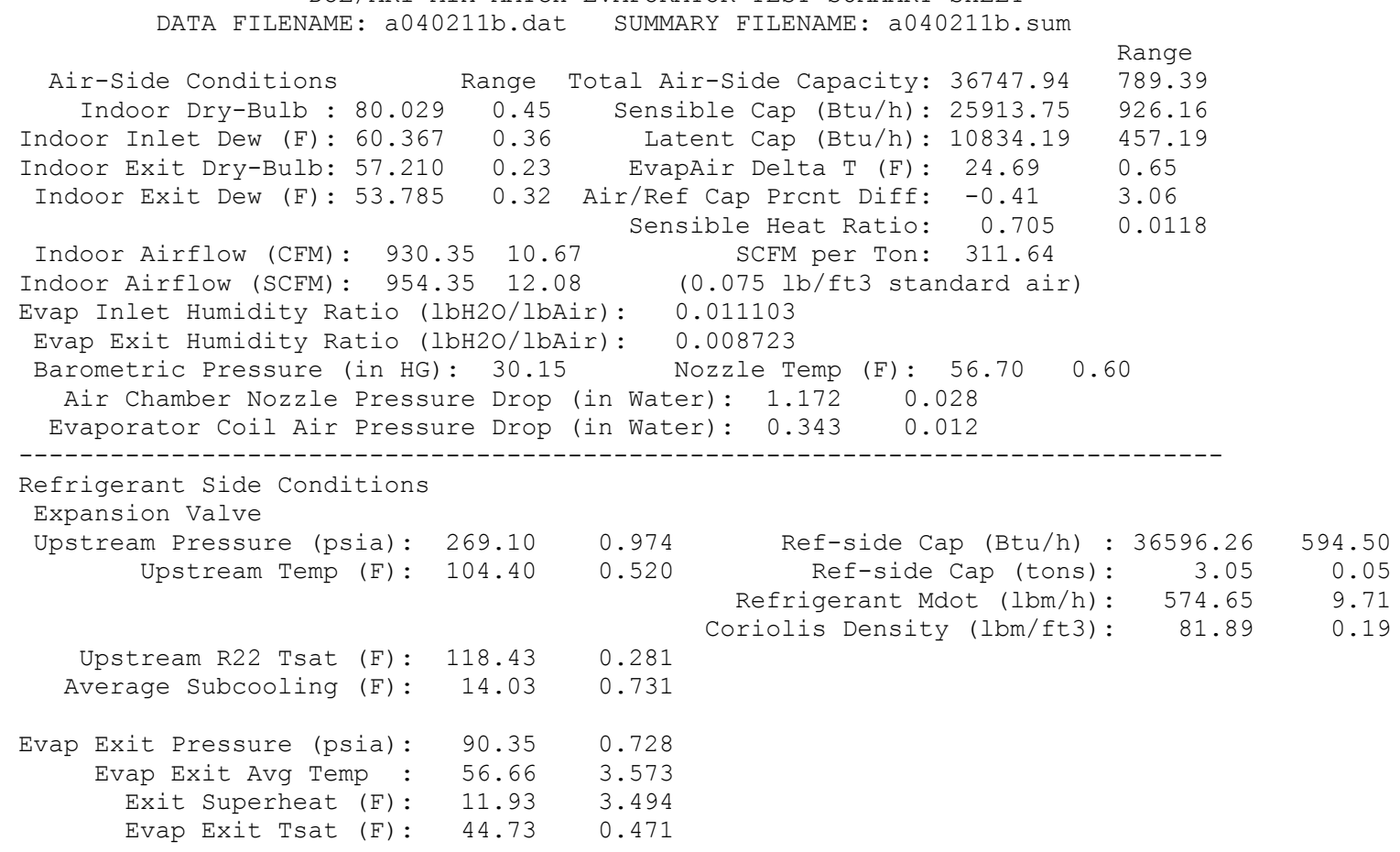




\section{Coil 4}

R22, A01138, No Fan

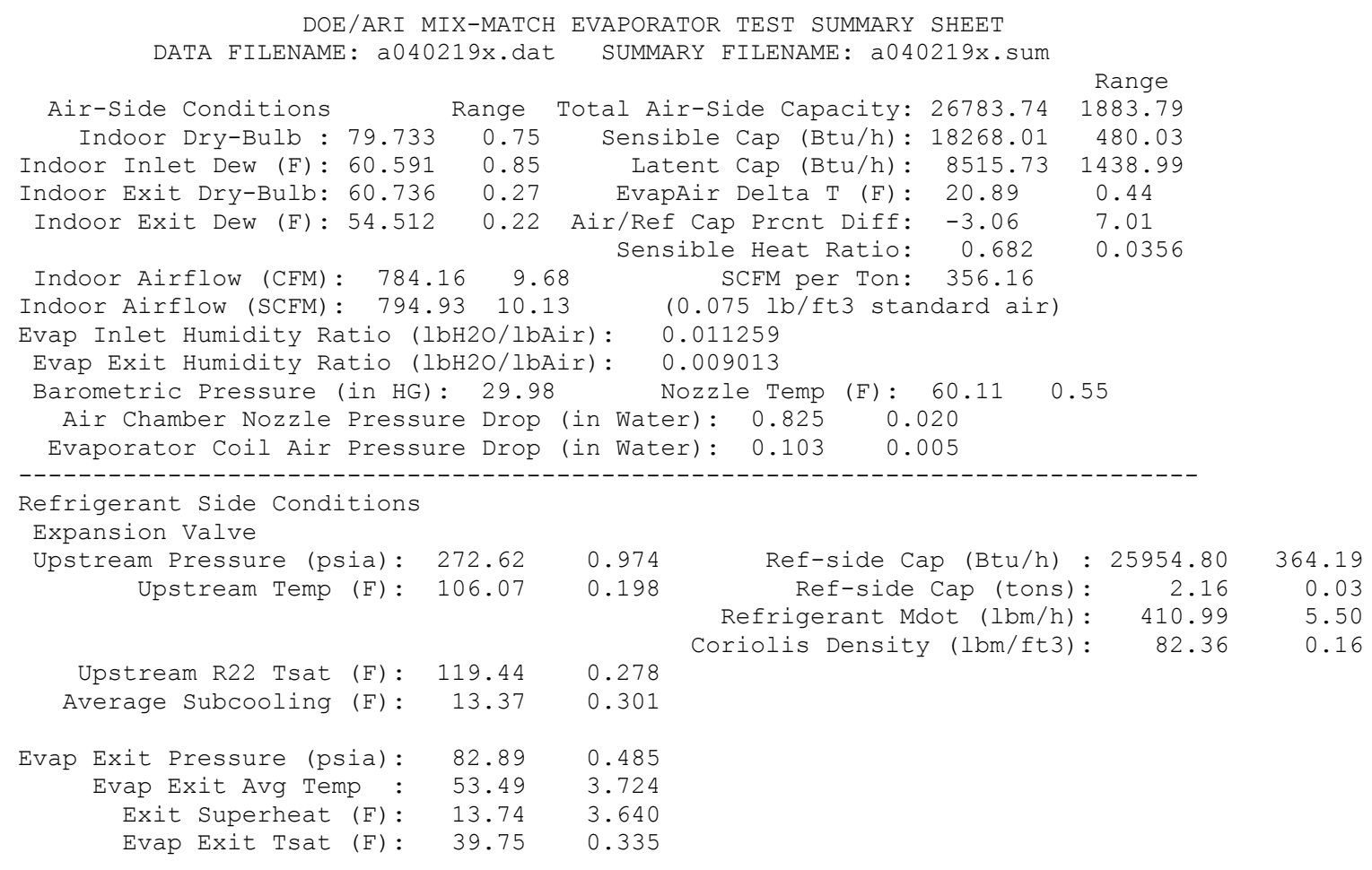


DOE/ARI MIX-MATCH EVAPORATOR TEST SUMMARY SHEET DATA FILENAME: a040223a.dat SUMMARY FILENAME: a040223a.sum

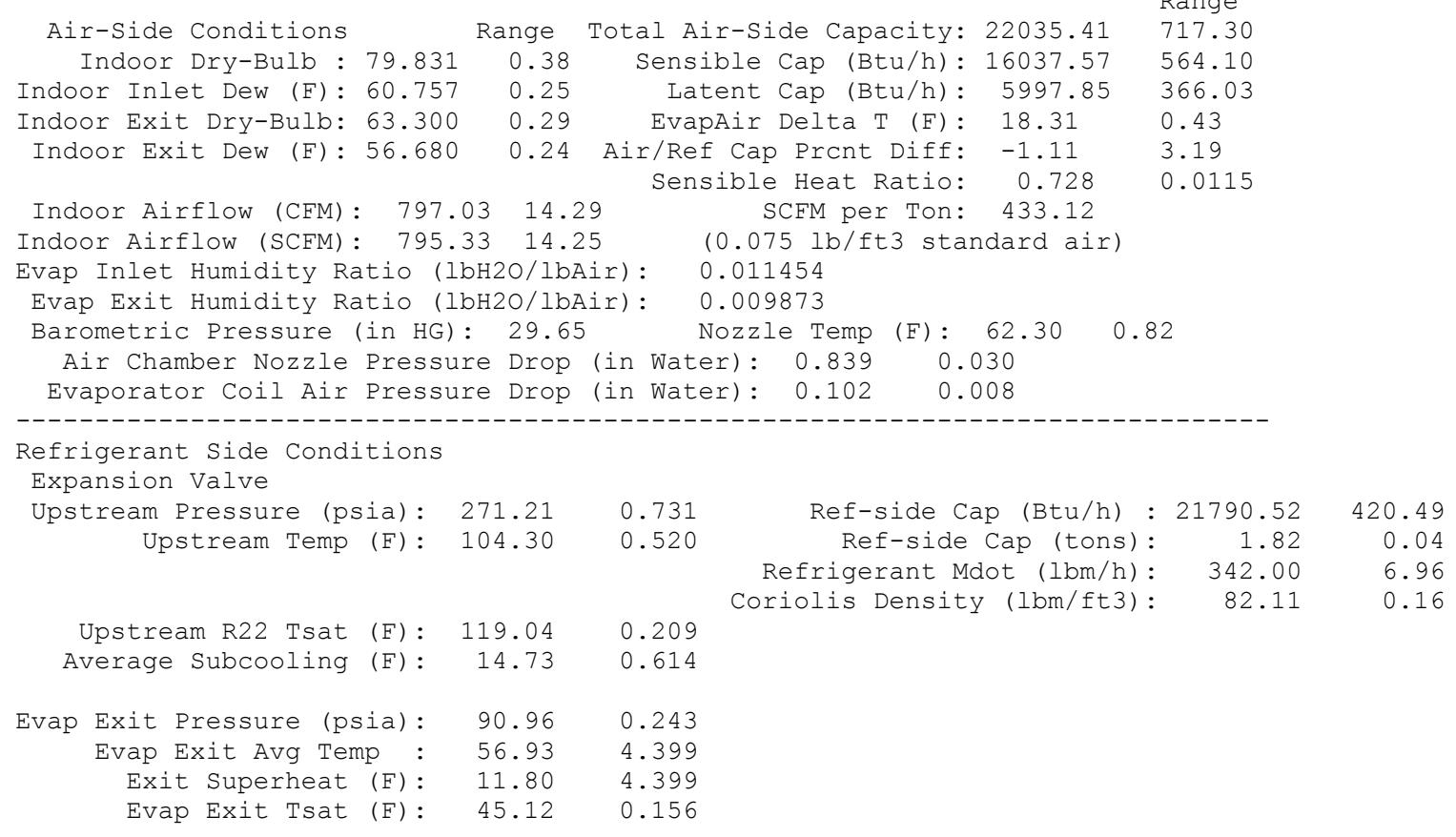


DOE/ARI MIX-MATCH EVAPORATOR TEST SUMMARY SHEET DATA FILENAME: a040225a.dat SUMMARY FILENAME: a040225a.sum

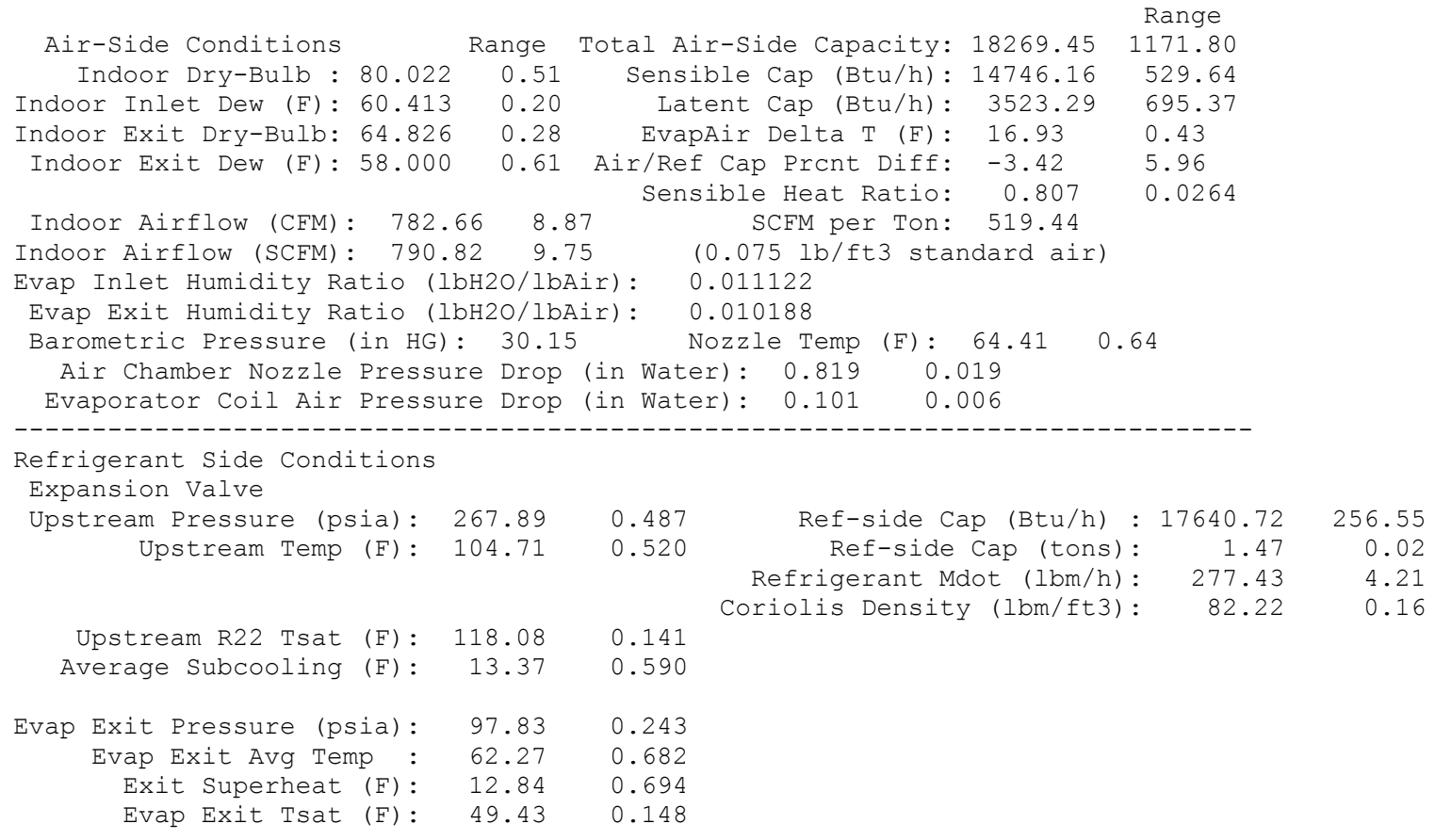


DOE/ARI MIX-MATCH EVAPORATOR TEST SUMMARY SHEET DATA FILENAME: a040226x.dat SUMMARY FILENAME: a040226x.sum

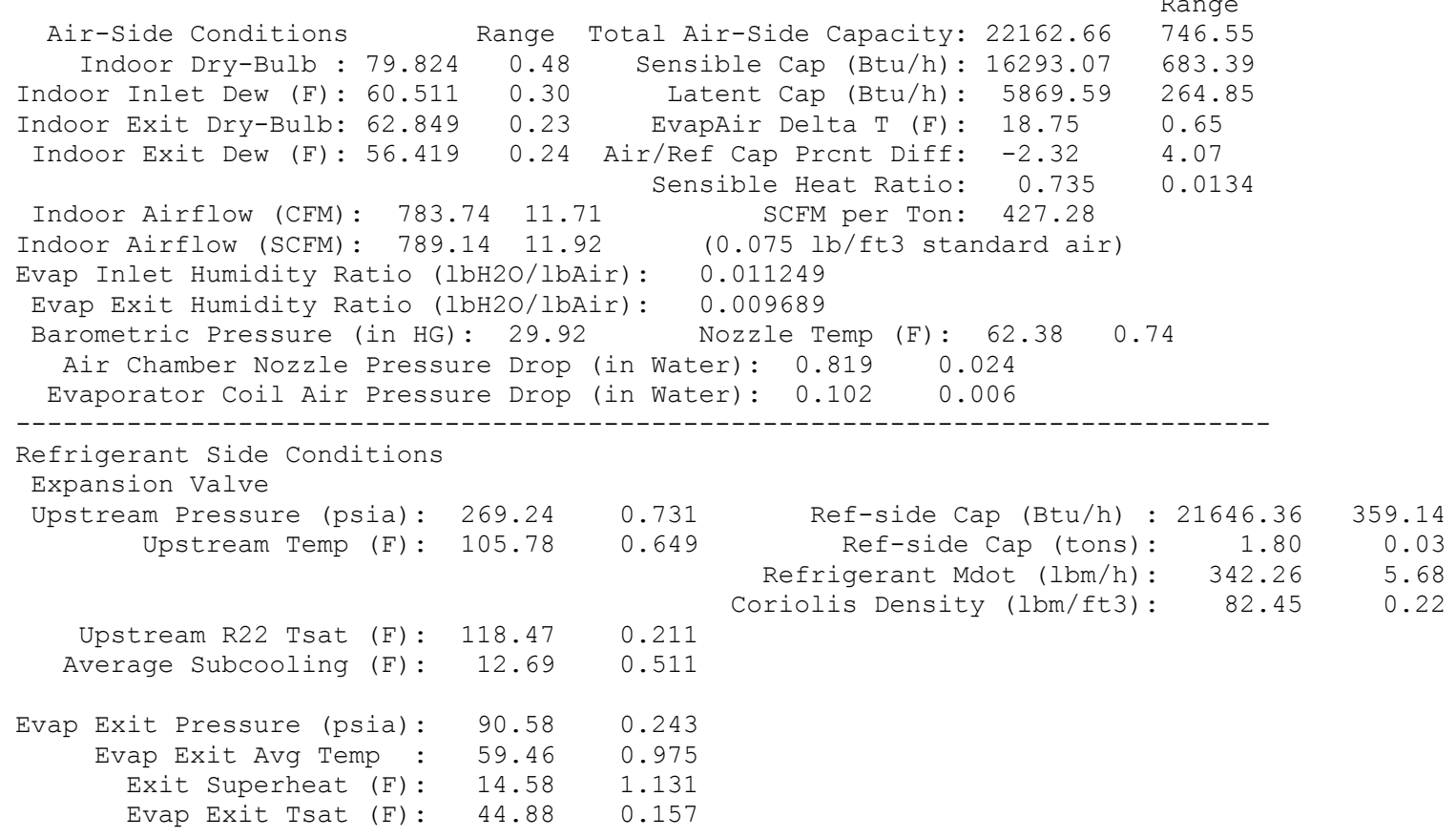


DOE/ARI MIX-MATCH EVAPORATOR TEST SUMMARY SHEET DATA FILENAME: b040225b.dat SUMMARY FILENAME: b040225b.sum

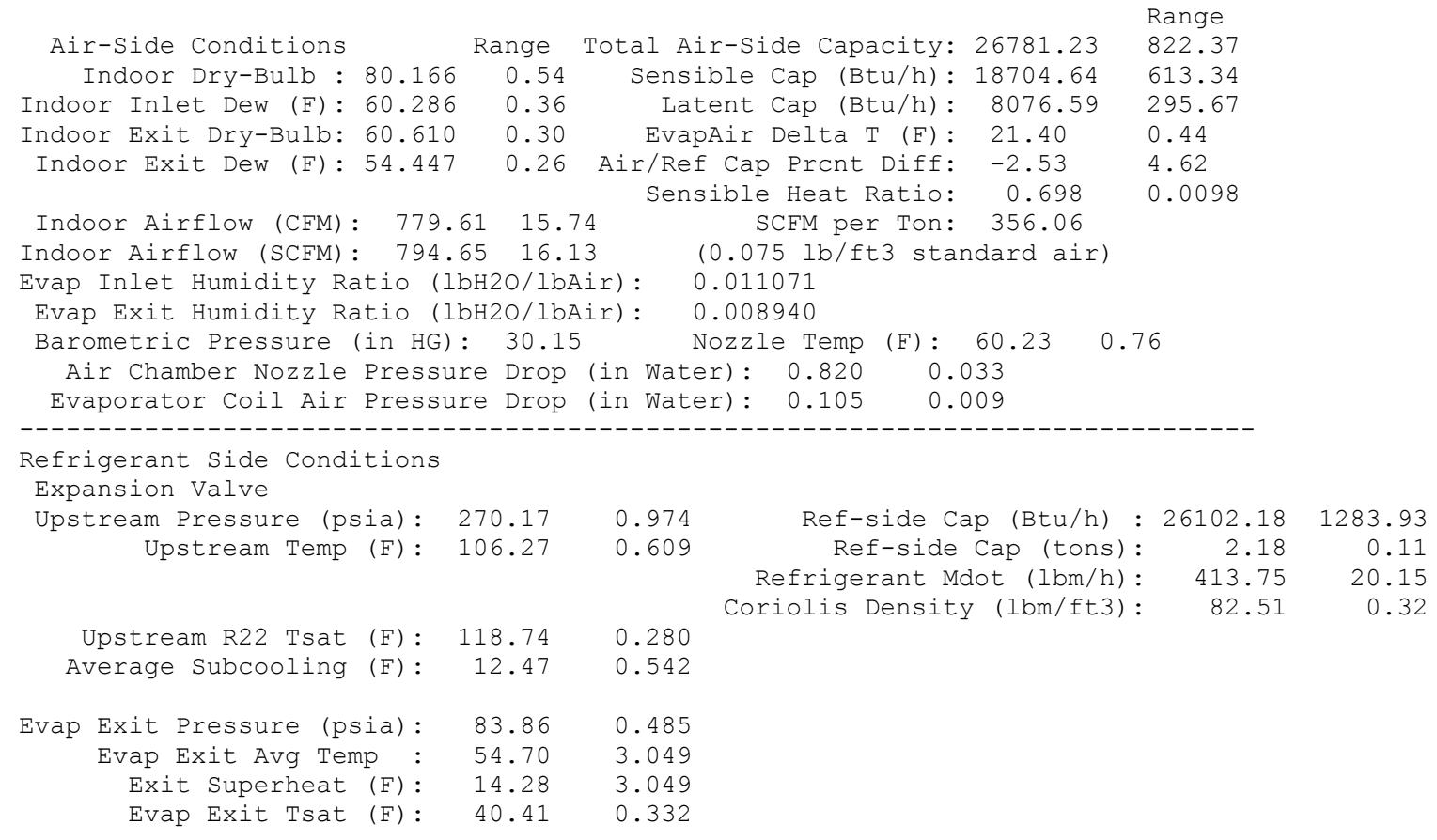


DOE/ARI MIX-MATCH EVAPORATOR TEST SUMMARY SHEET DATA FILENAME: b040226b. dat SUMMARY FILENAME: b040226b.sum

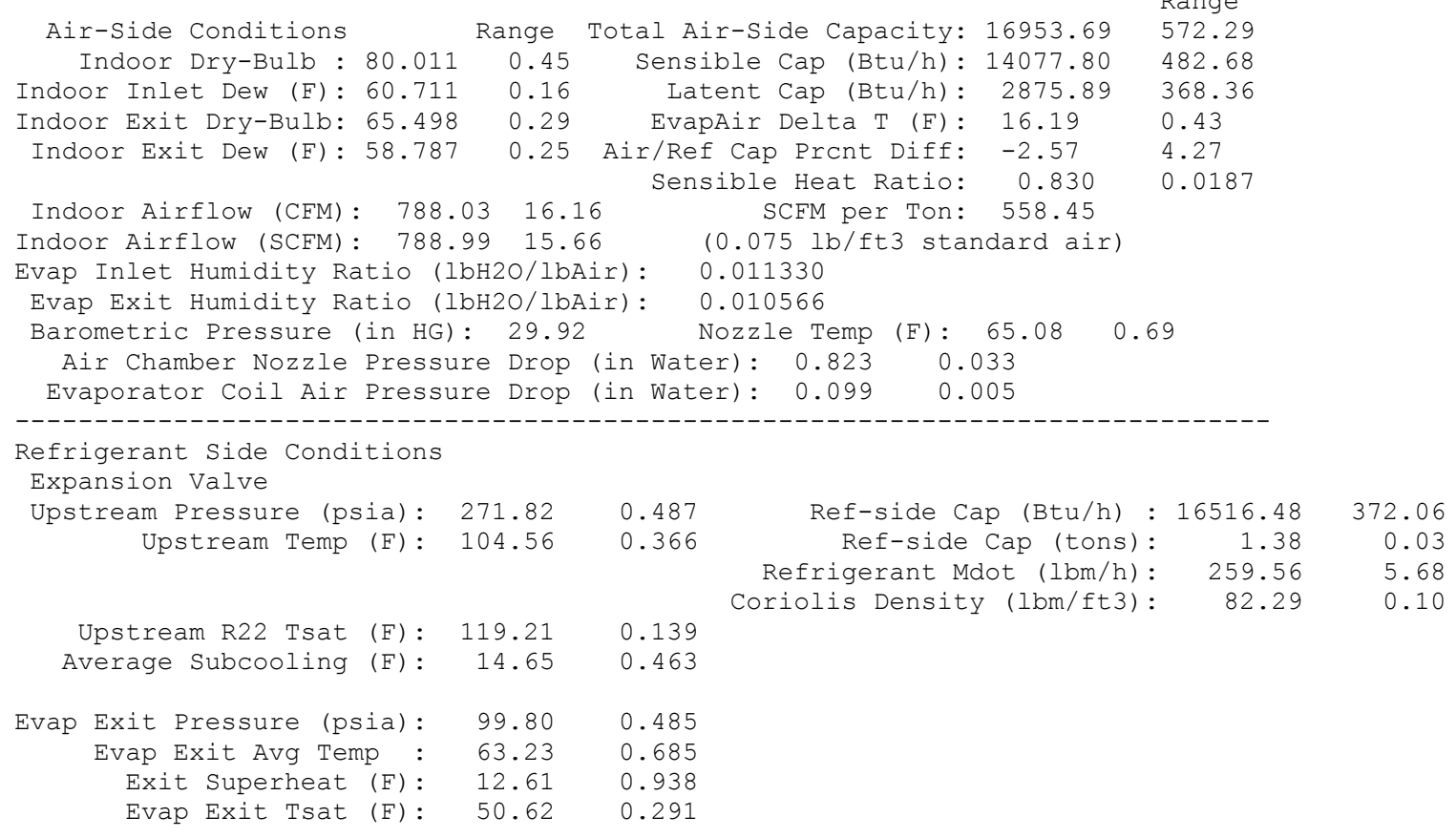




\section{Coil 5}

R22, A01060, Fan: 273 Watts

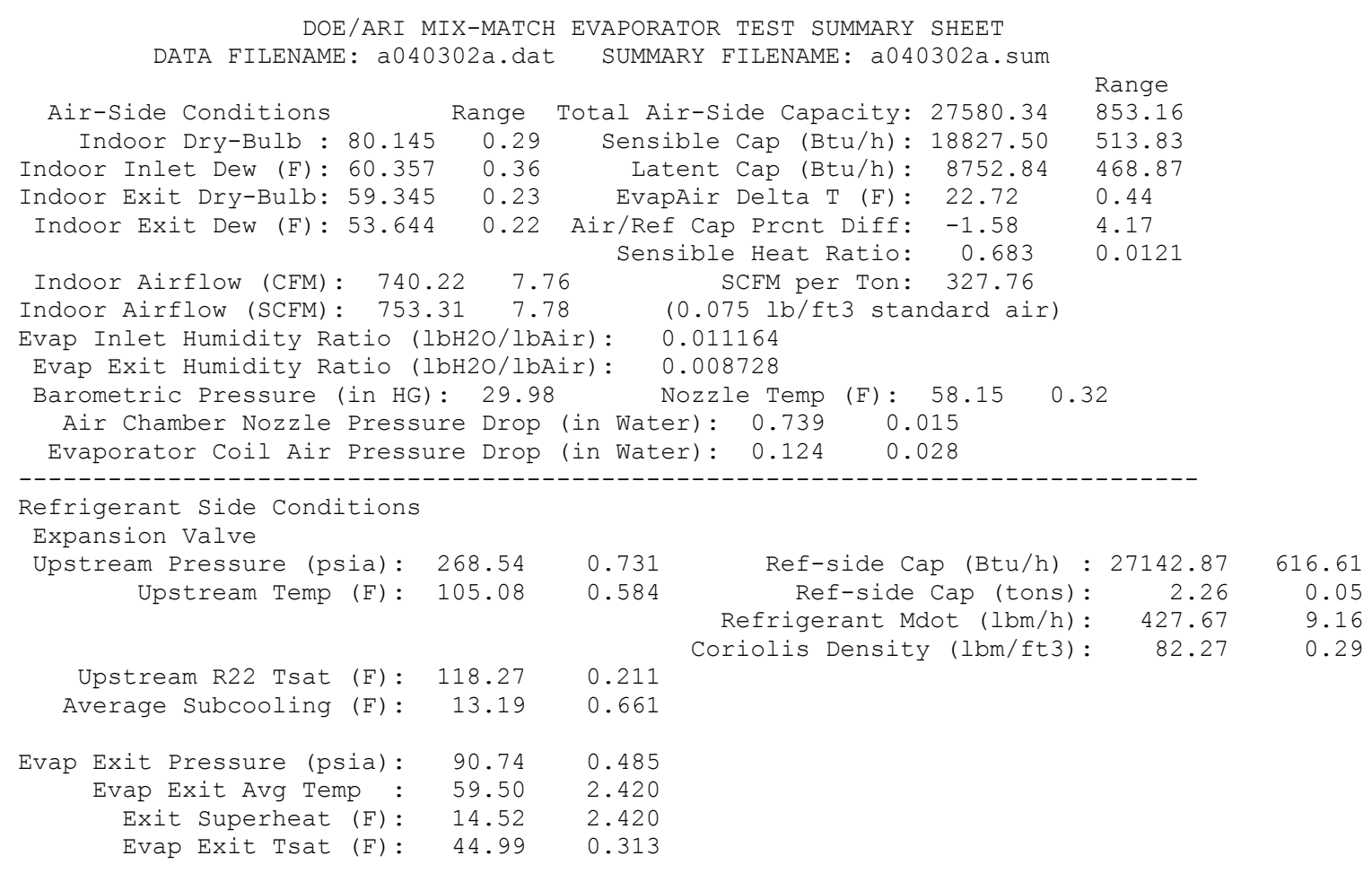


DOE/ARI MIX-MATCH EVAPORATOR TEST SUMMARY SHEET DATA FILENAME: a040303a.dat SUMMARY FILENAME: a040303a.sum

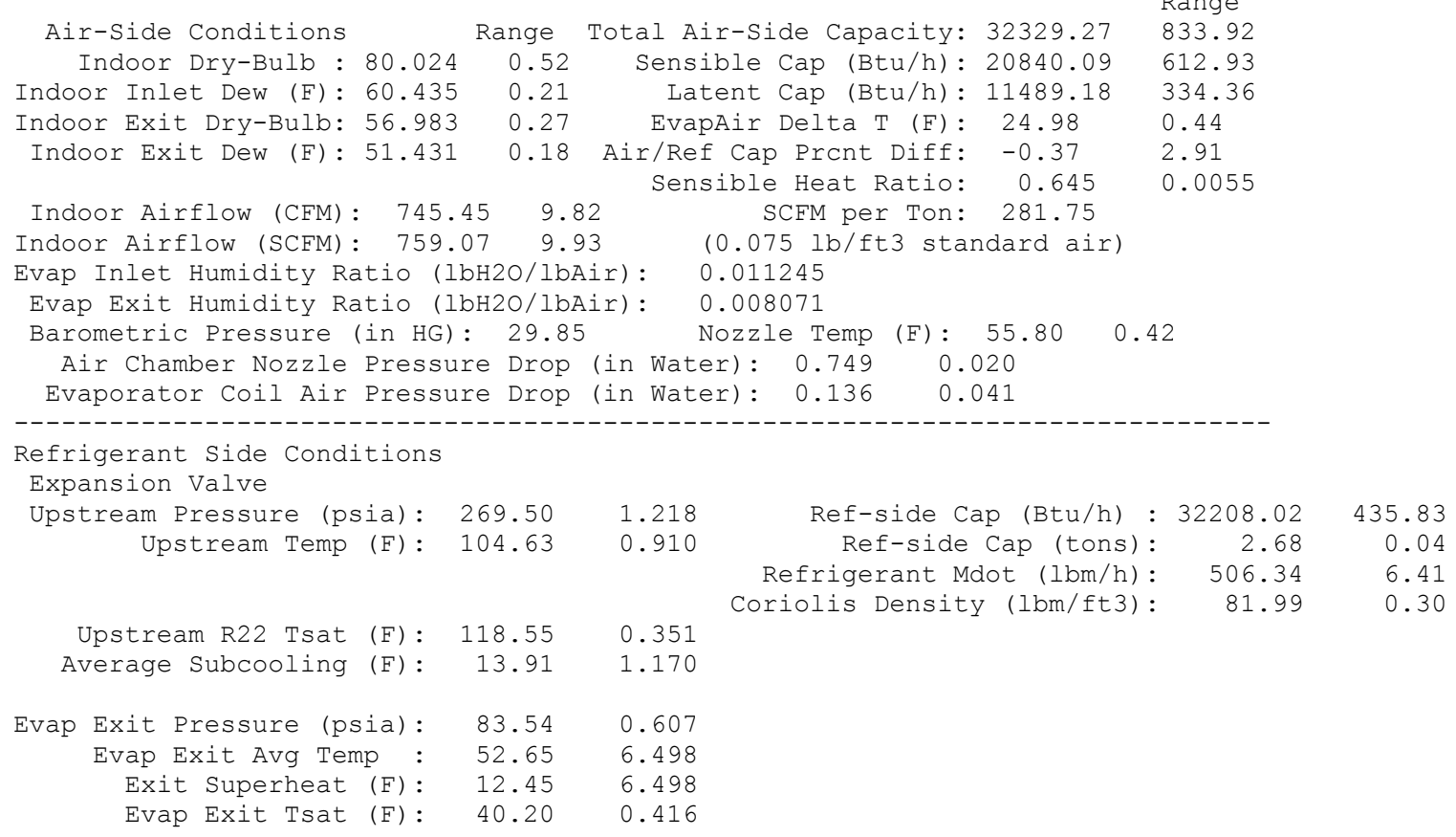


DOE/ARI MIX-MATCH EVAPORATOR TEST SUMMARY SHEET DATA FILENAME: a040304a.dat SUMMARY FILENAME: a040304a.sum

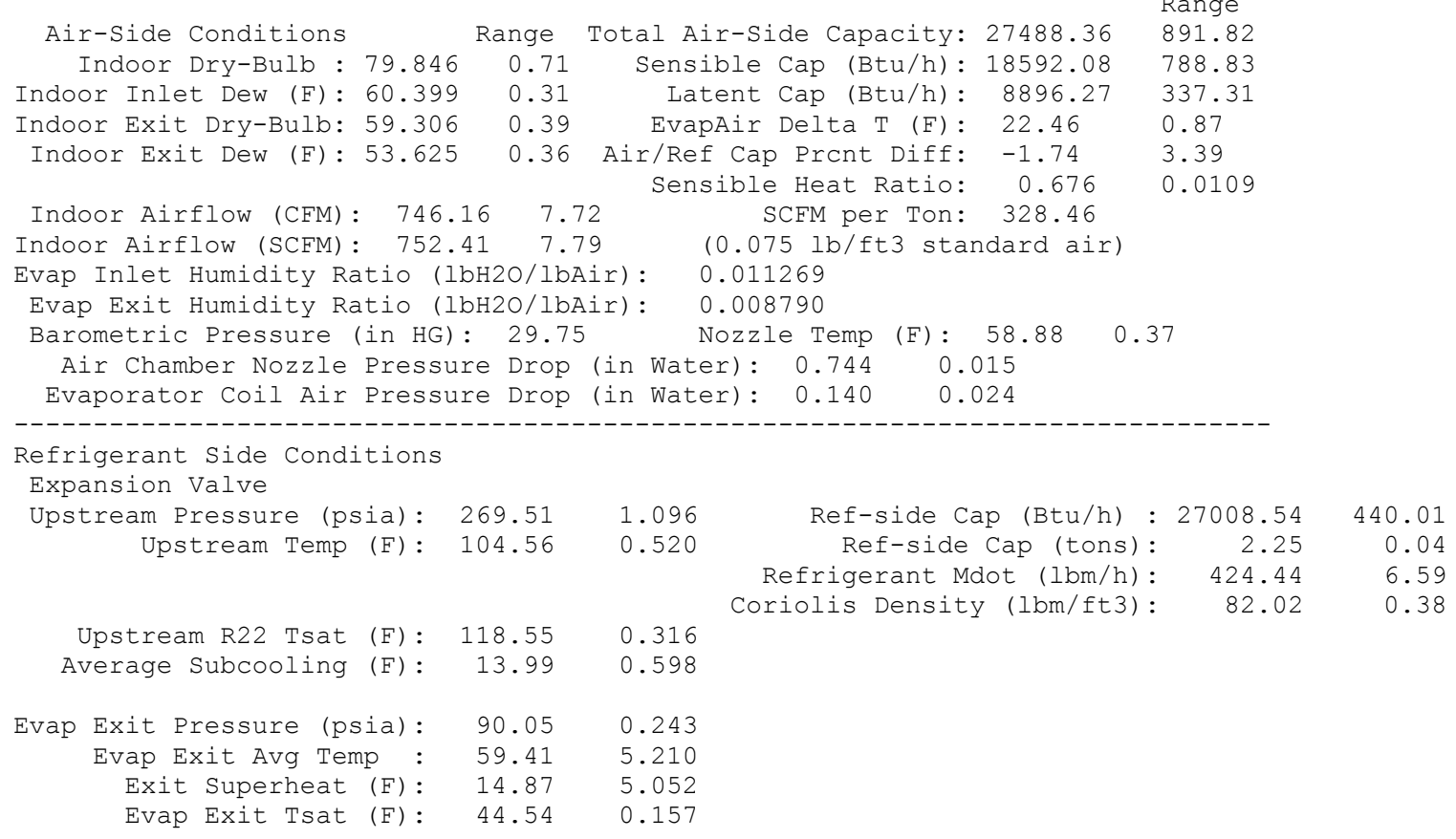


DOE/ARI MIX-MATCH EVAPORATOR TEST SUMMARY SHEET DATA FILENAME: b040303b. dat SUMMARY FILENAME: b040303b.sum

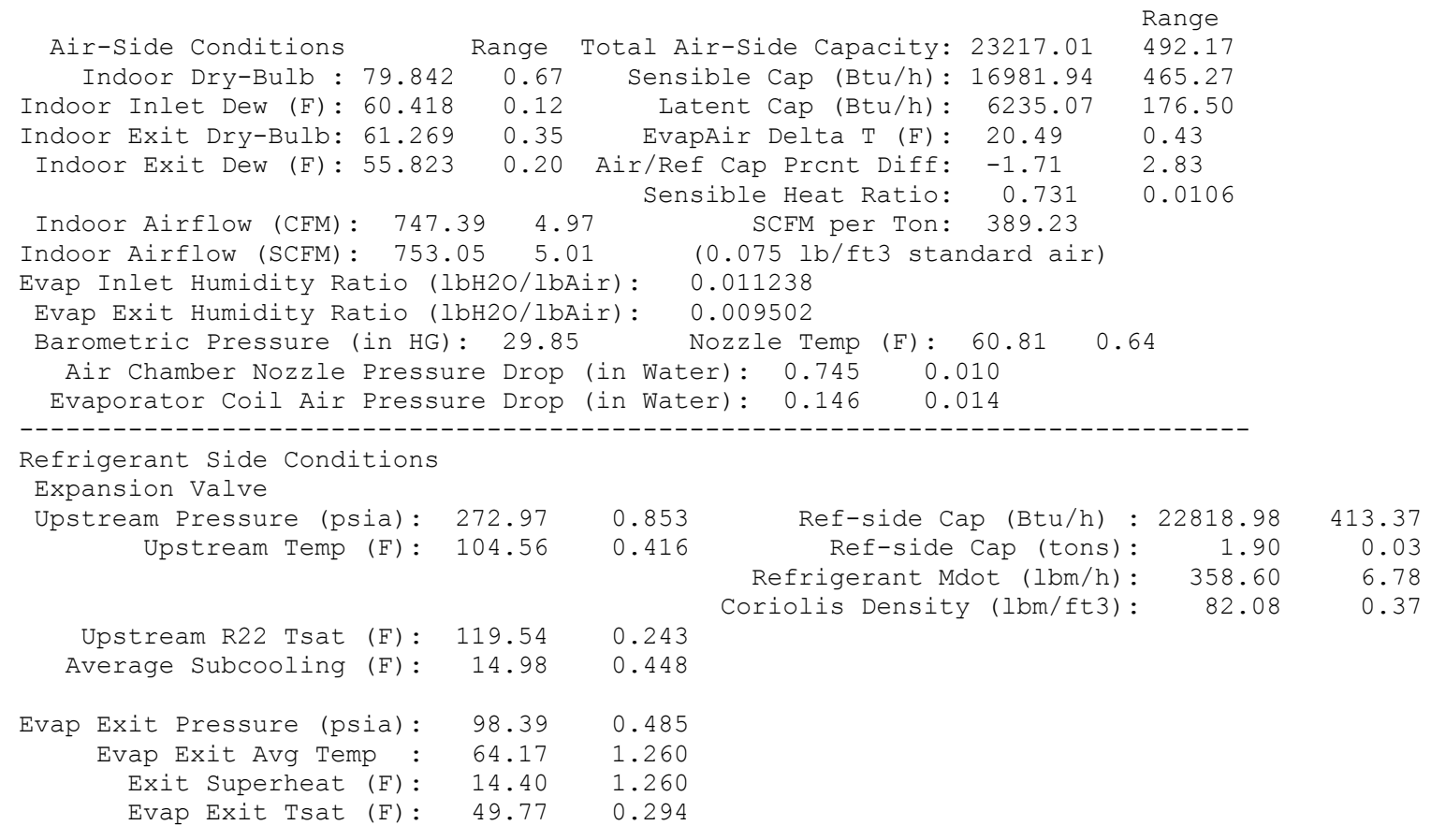


DOE/ARI MIX-MATCH EVAPORATOR TEST SUMMARY SHEET DATA FILENAME: a040305a.dat SUMMARY FILENAME: a040305a.sum

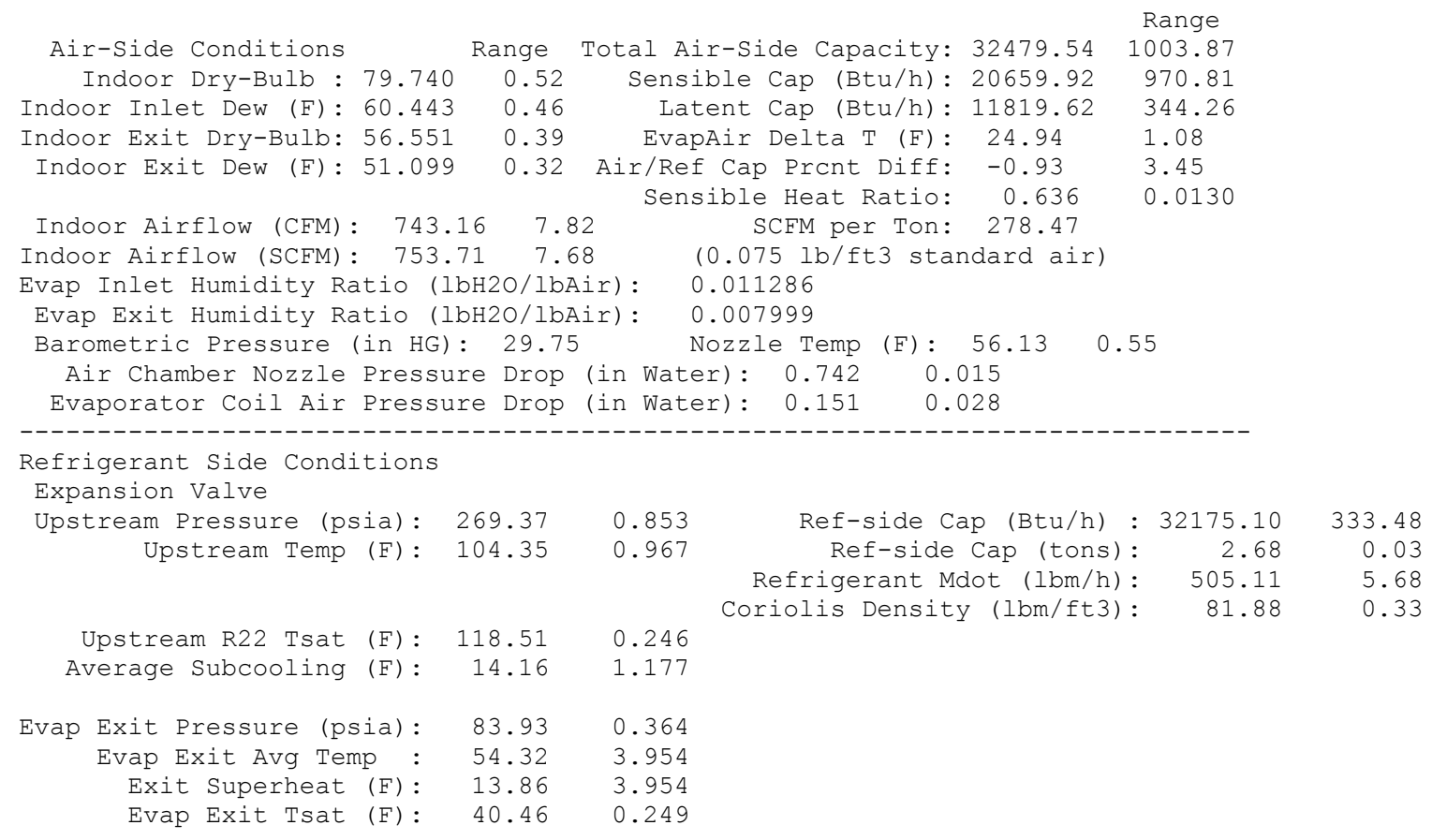


DOE/ARI MIX-MATCH EVAPORATOR TEST SUMMARY SHEET DATA FILENAME: a040308a.dat SUMMARY FILENAME: a040308a.sum

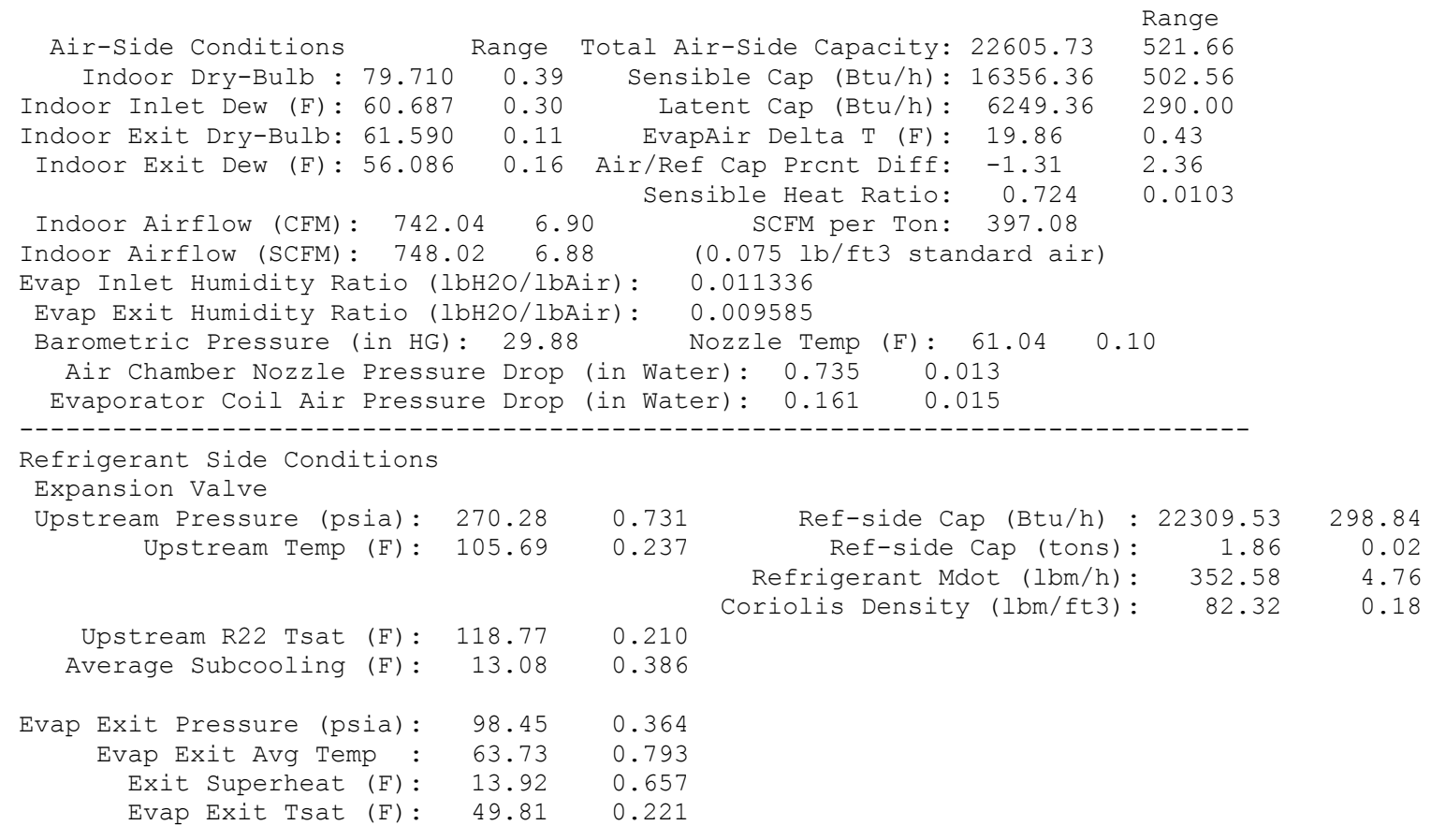




\section{Coil 6}

\section{R22, A01125, Fan: 768 Watts}

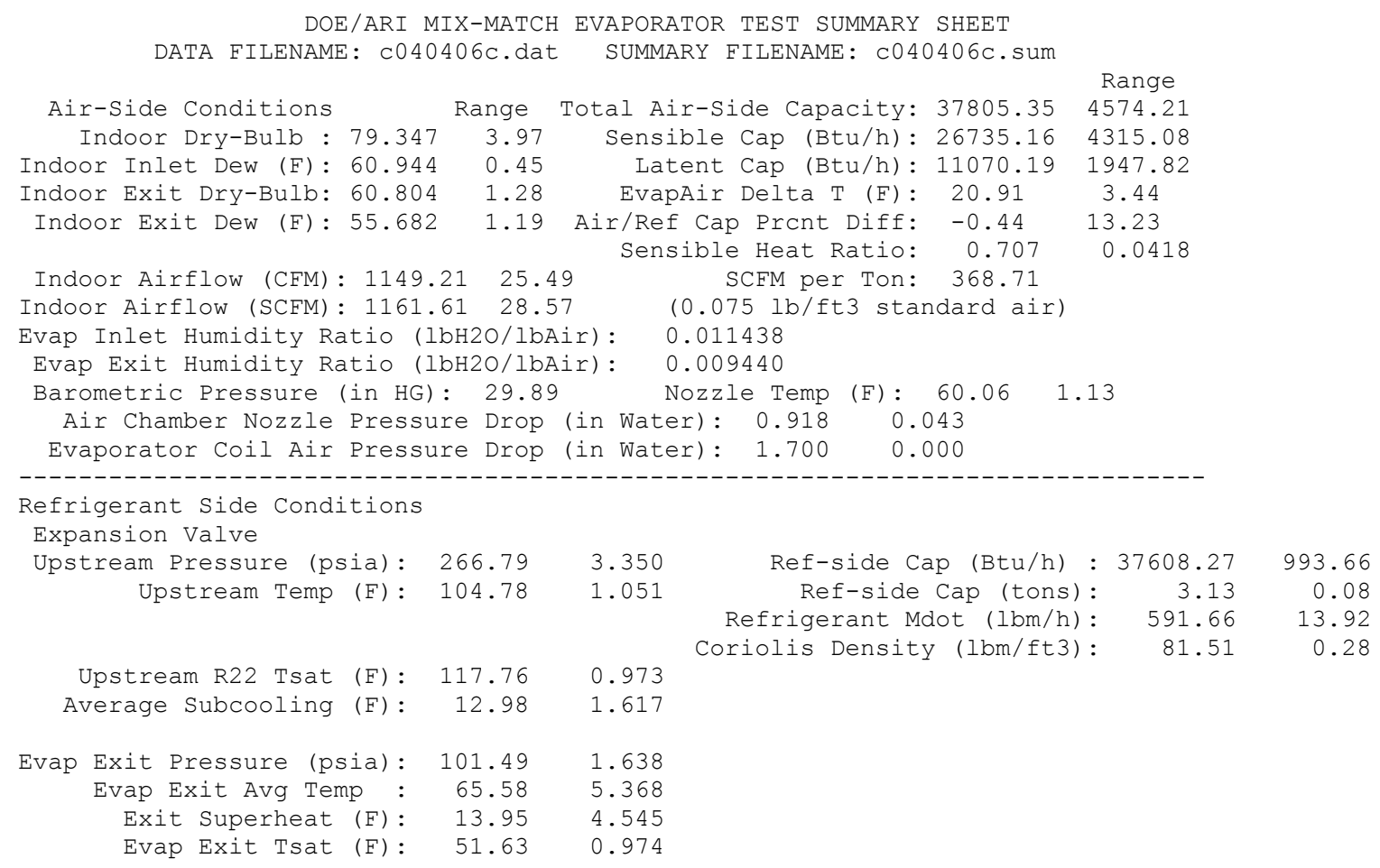


DOE/ARI MIX-MATCH EVAPORATOR TEST SUMMARY SHEET DATA FILENAME: a040407a.dat SUMMARY FILENAME: a040407a.sum

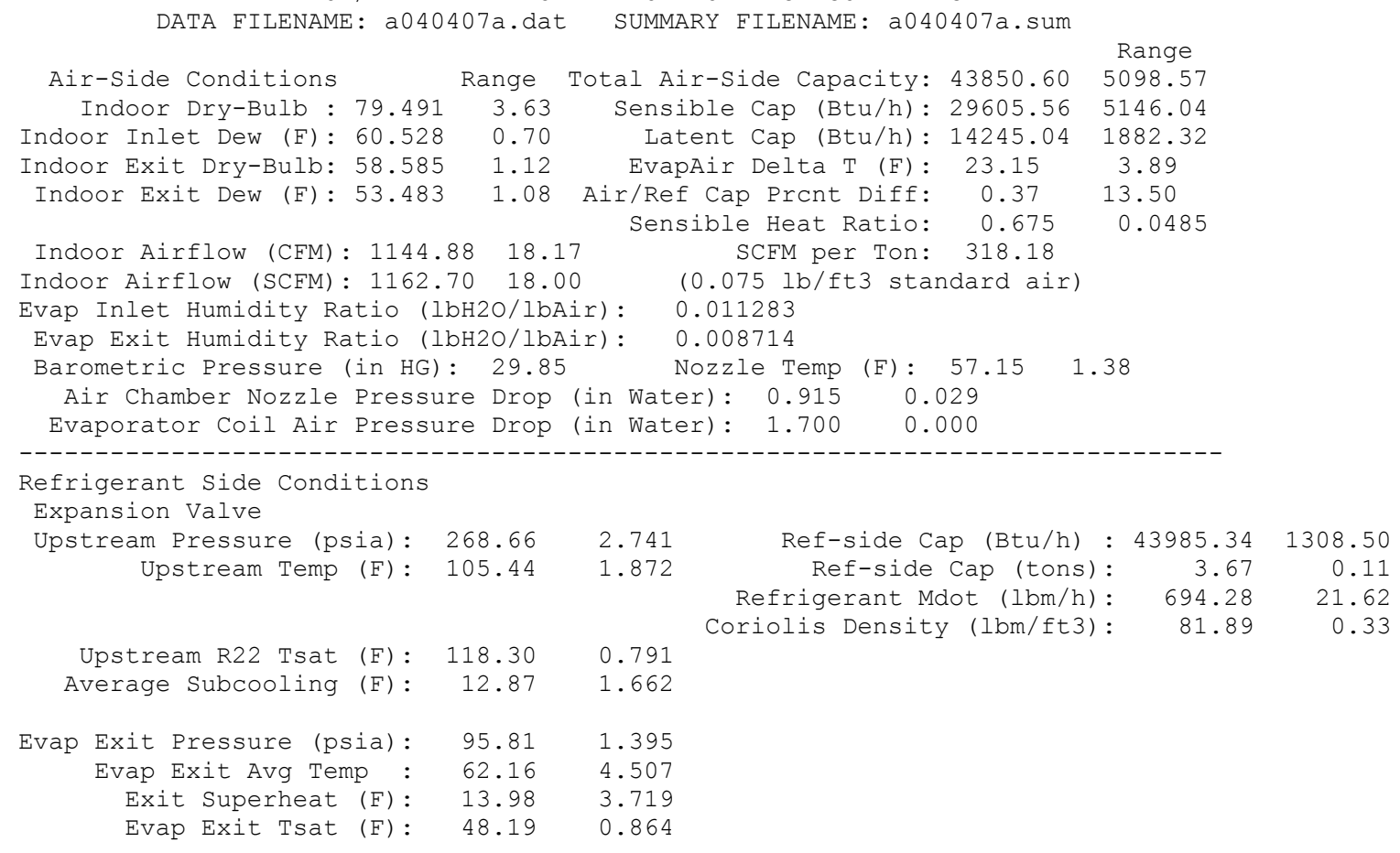


DOE/ARI MIX-MATCH EVAPORATOR TEST SUMMARY SHEET DATA FILENAME: d040406d.dat SUMMARY FILENAME: d040406d.sum

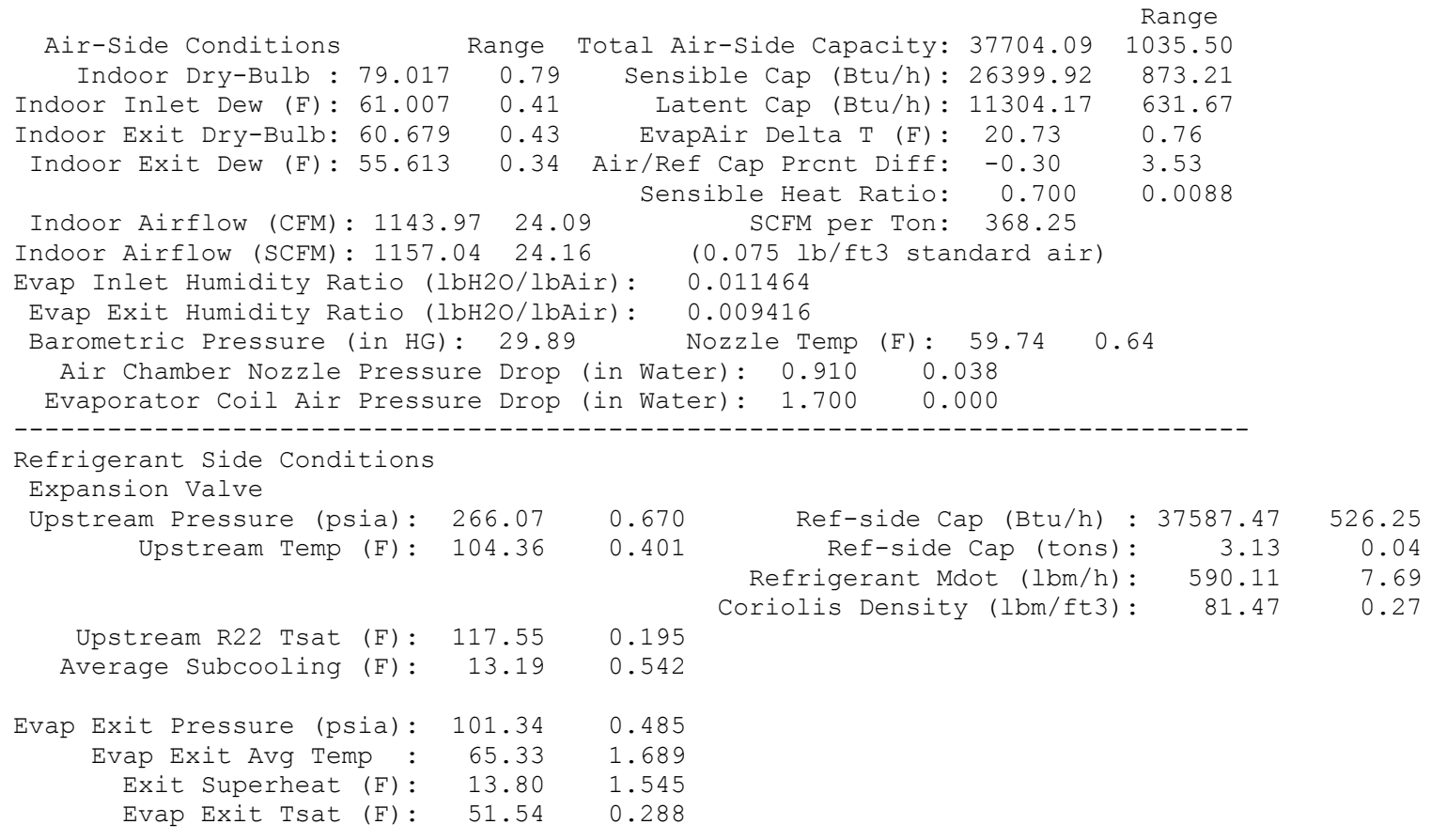




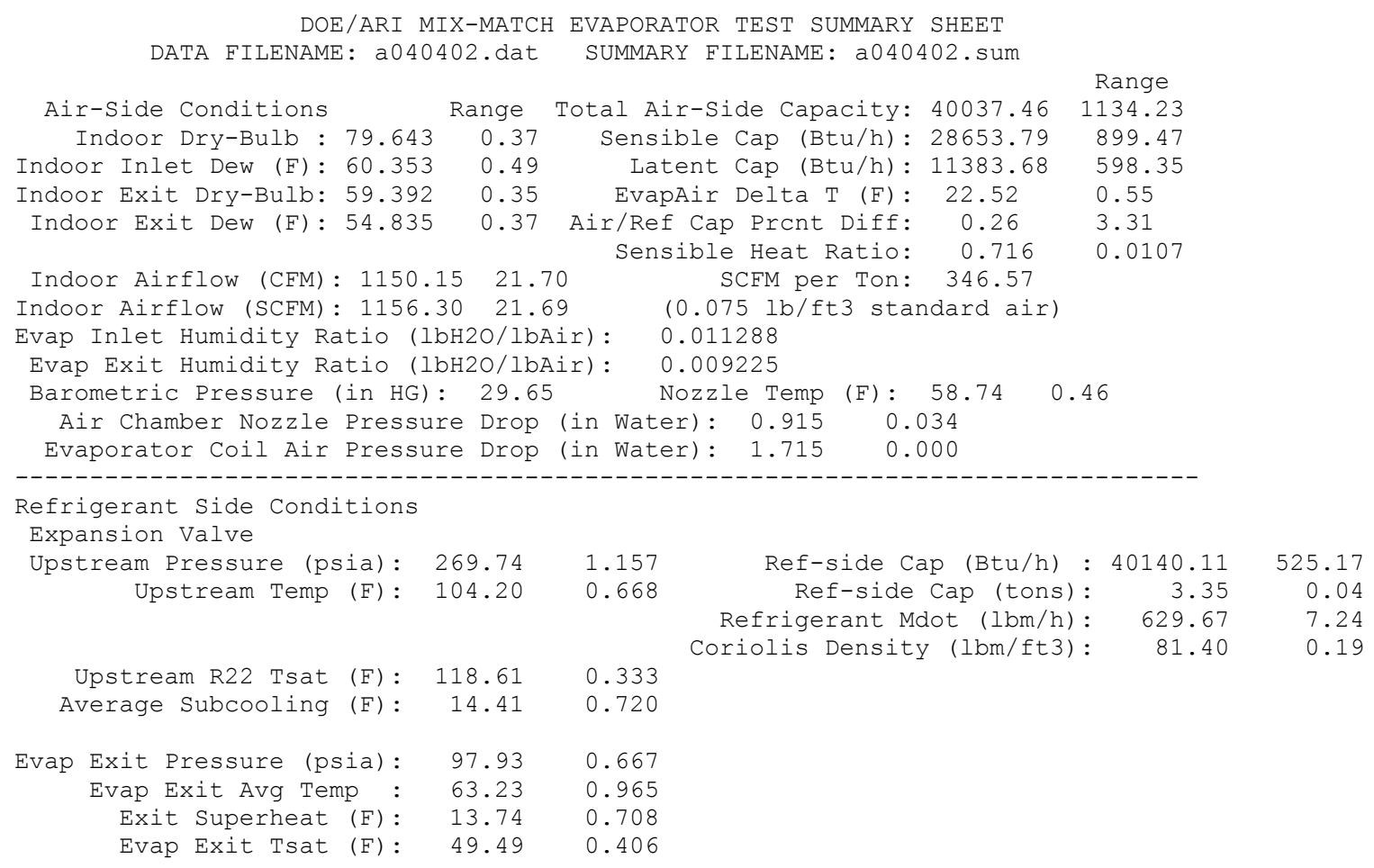


DOE/ARI MIX-MATCH EVAPORATOR TEST SUMMARY SHEET DATA FILENAME: a040406a.dat SUMMARY FILENAME: a040406a.sum

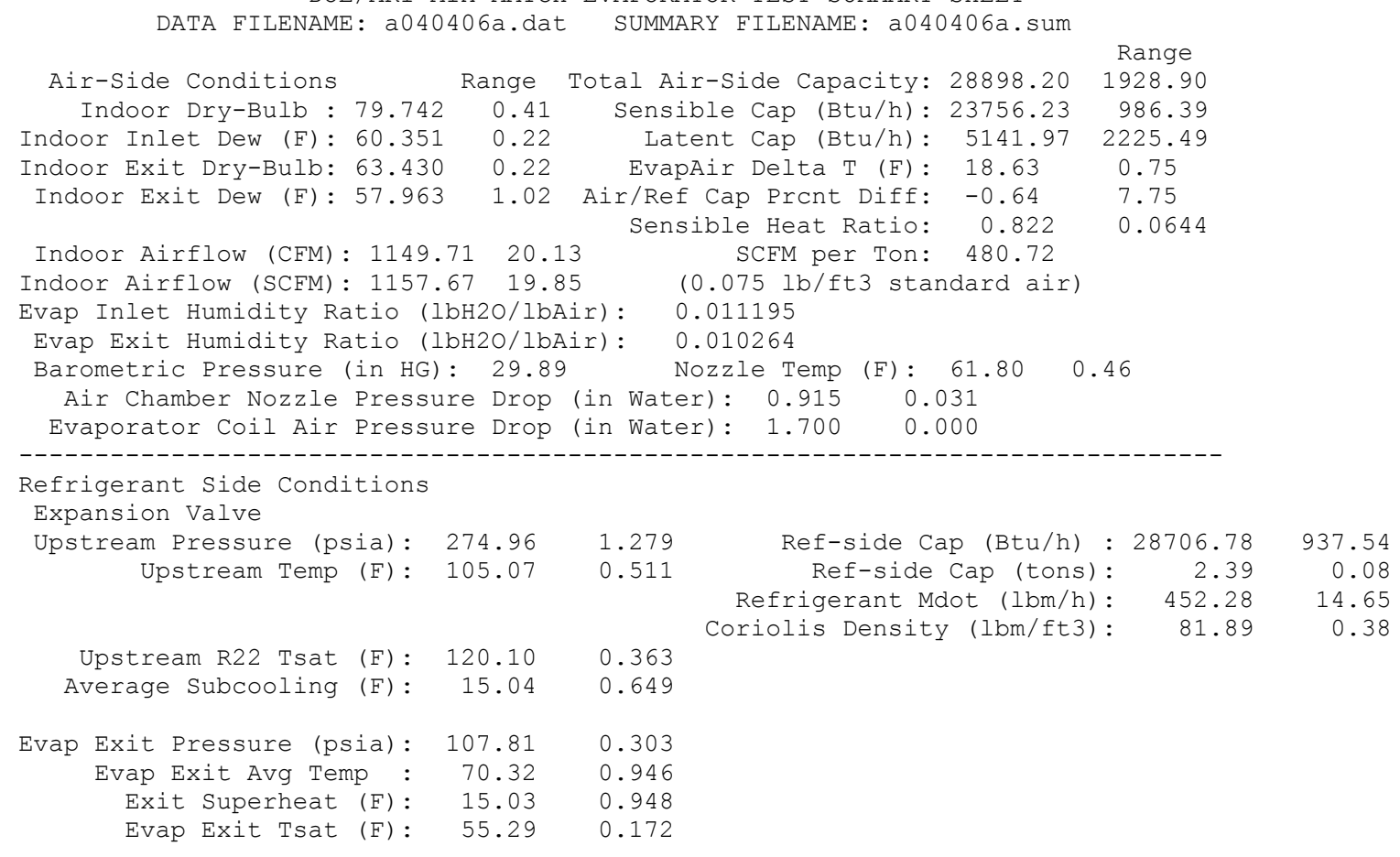


DOE/ARI MIX-MATCH EVAPORATOR TEST SUMMARY SHEET DATA FILENAME: b040402b. dat SUMMARY FILENAME: b040402b.sum

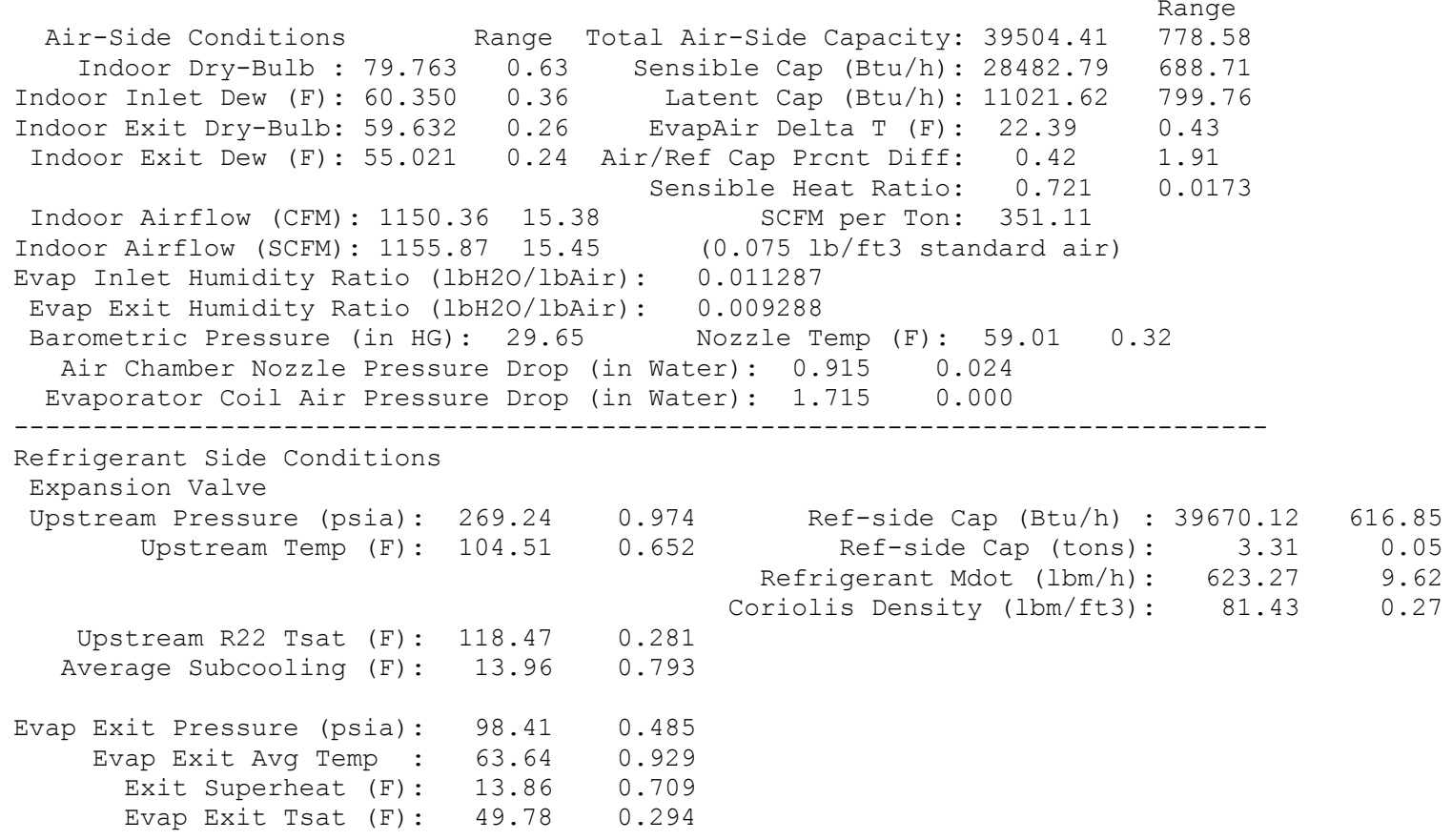


DOE/ARI MIX-MATCH EVAPORATOR TEST SUMMARY SHEET DATA FILENAME: b040407b. dat SUMMARY FILENAME: b040407b.sum

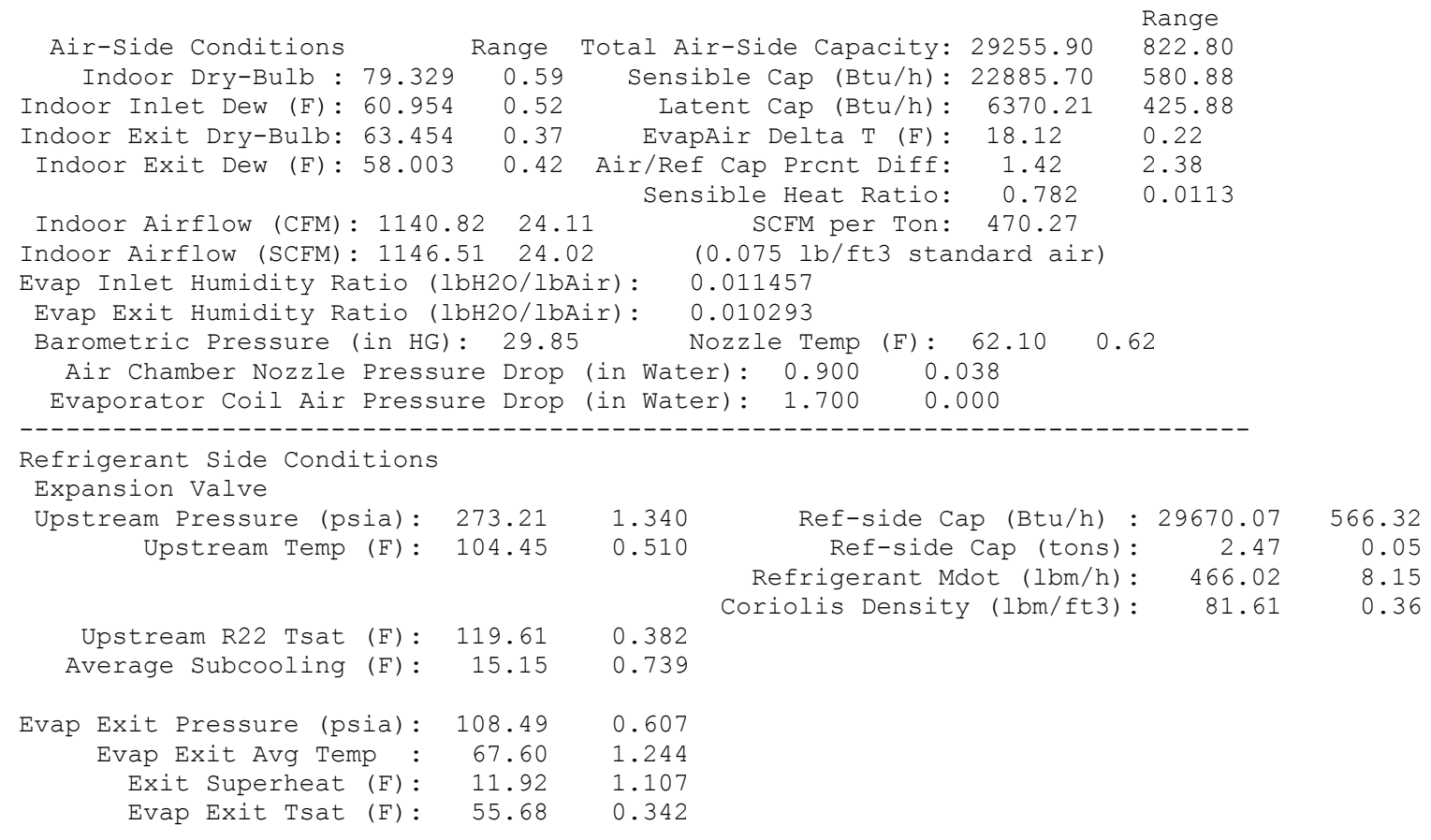


DOE/ARI MIX-MATCH EVAPORATOR TEST SUMMARY SHEET DATA FILENAME: a040410a.dat SUMMARY FILENAME: a040410a.sum

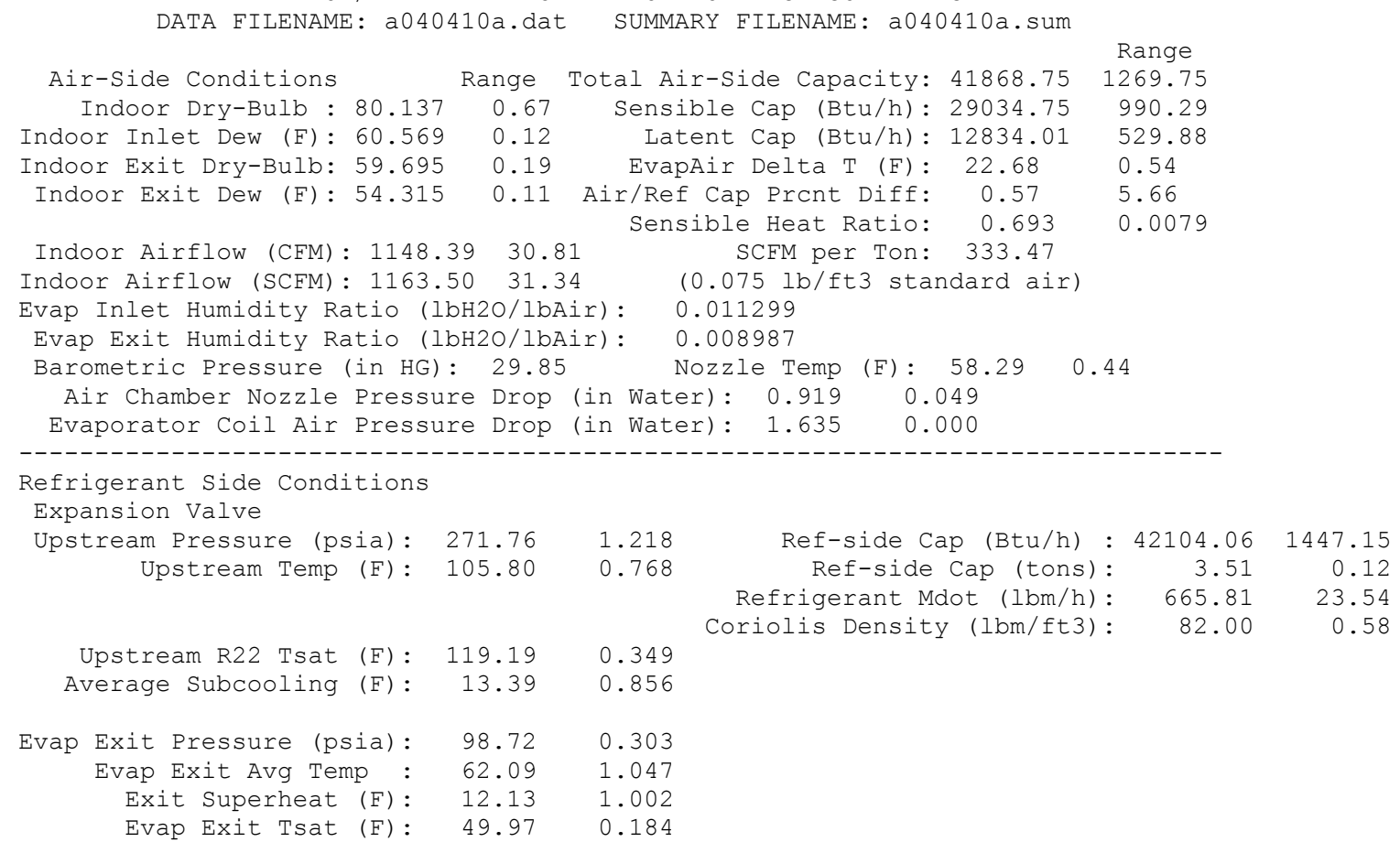




\section{Coil 7}

R22, H5326, No Fan

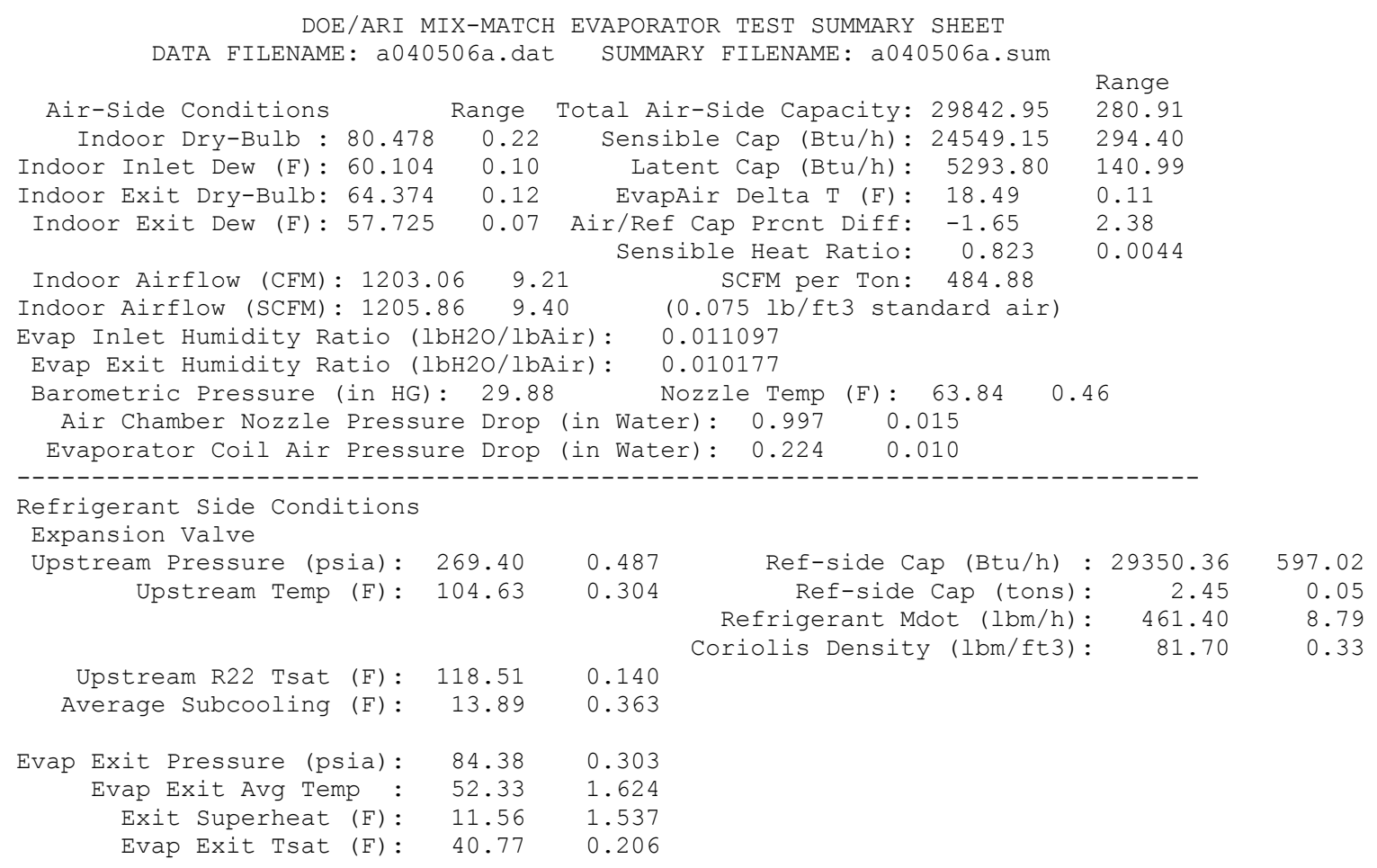


DOE/ARI MIX-MATCH EVAPORATOR TEST SUMMARY SHEET DATA FILENAME: a040507a.dat SUMMARY FILENAME: a040507a.sum

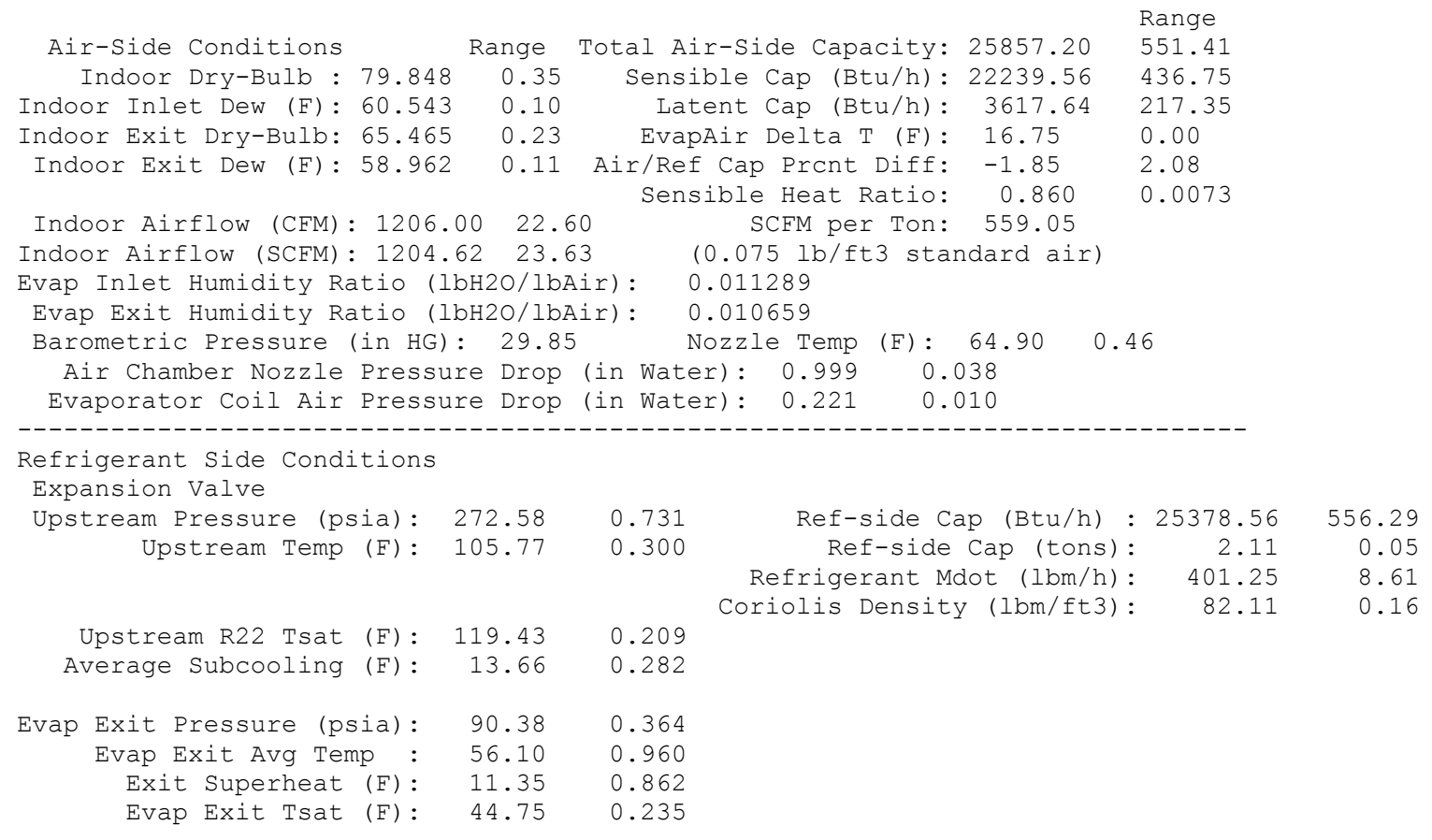


DOE/ARI MIX-MATCH EVAPORATOR TEST SUMMARY SHEET DATA FILENAME: a040510a.dat SUMMARY FILENAME: a040510a.sum

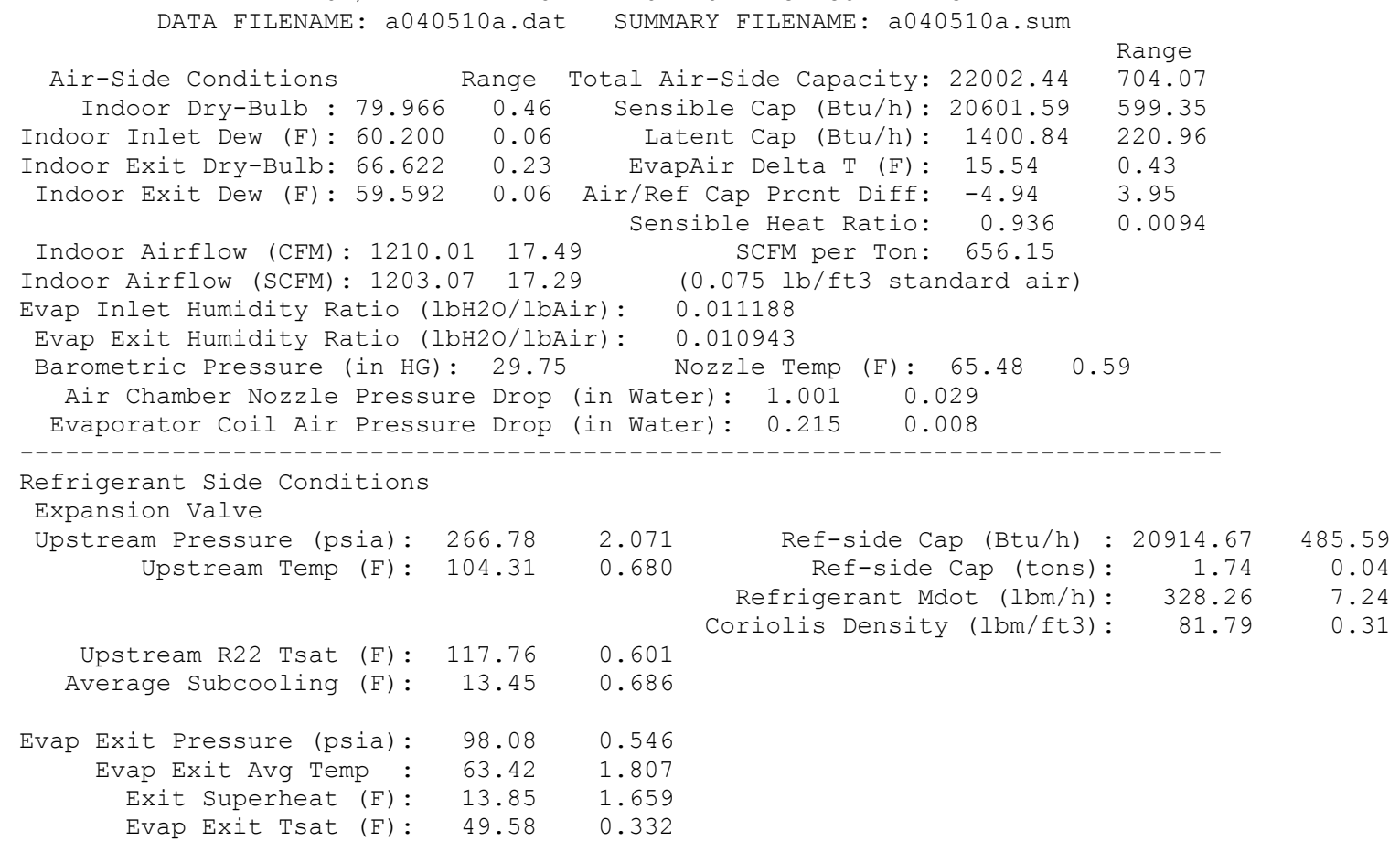


DOE/ARI MIX-MATCH EVAPORATOR TEST SUMMARY SHEET DATA FILENAME: a040513a.dat SUMMARY FILENAME: a040513a.sum

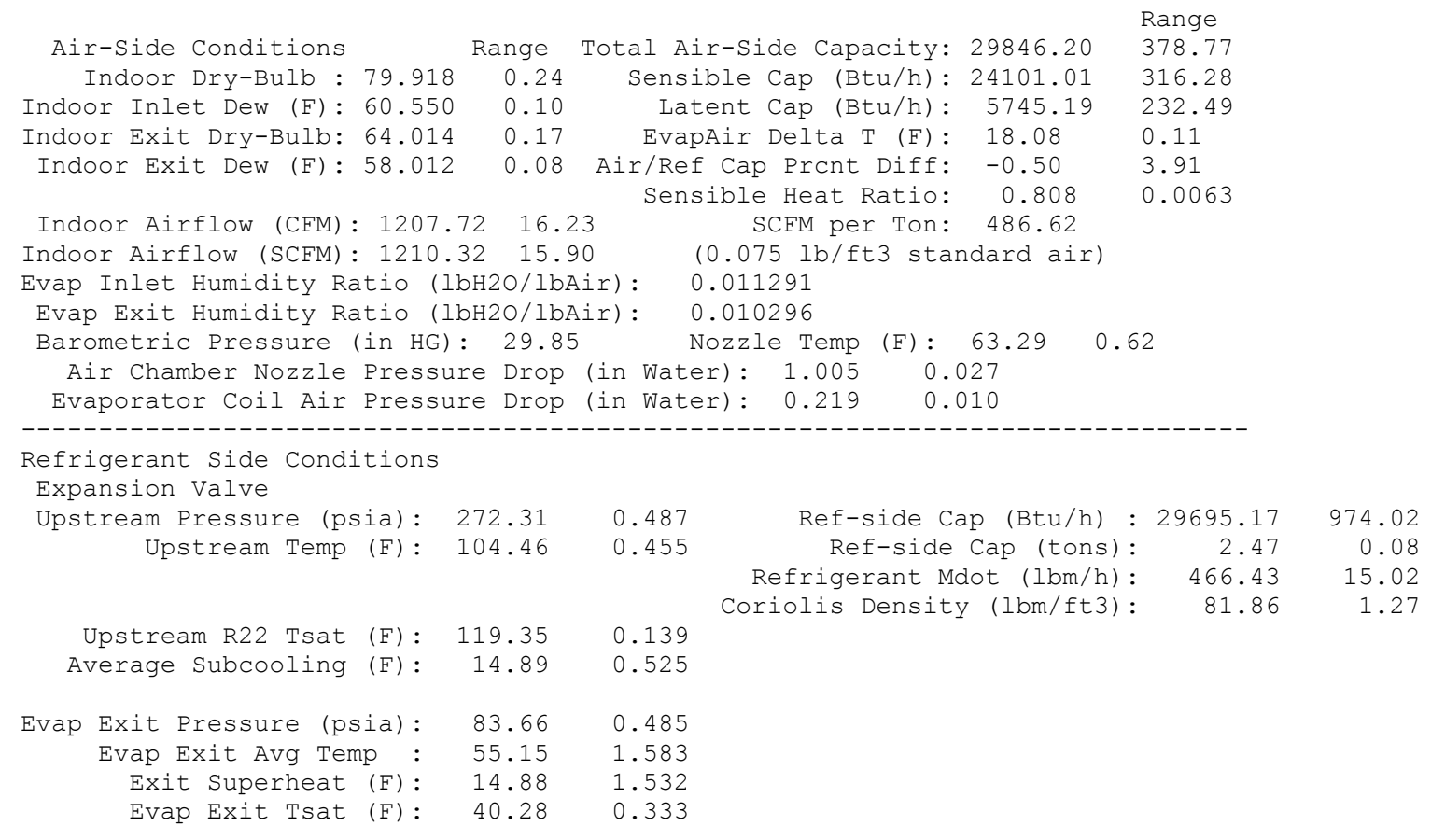


DOE/ARI MIX-MATCH EVAPORATOR TEST SUMMARY SHEET DATA FILENAME: b040507b. dat SUMMARY FILENAME: b040507b.sum

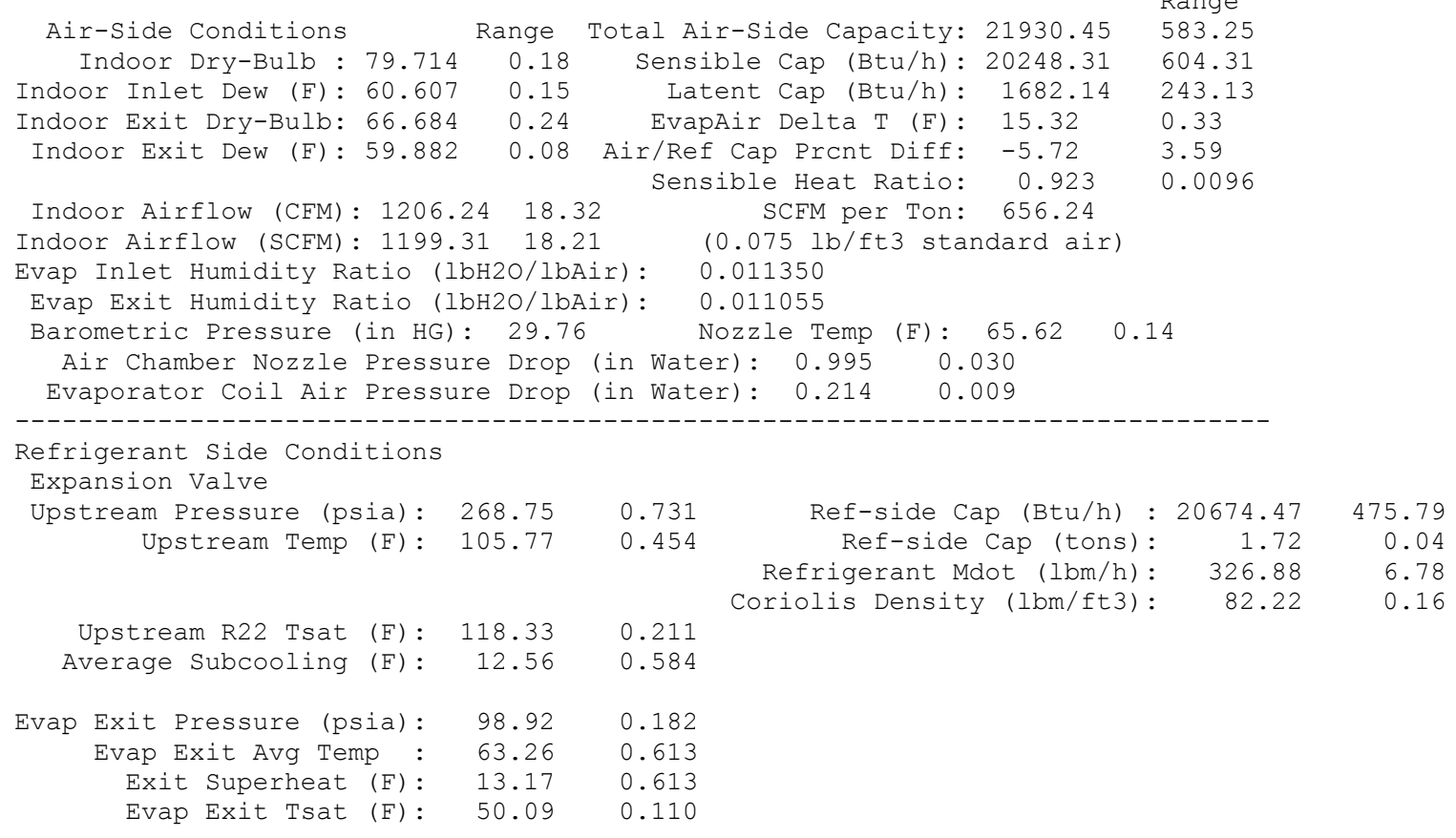


DOE/ARI MIX-MATCH EVAPORATOR TEST SUMMARY SHEET DATA FILENAME: b040511b. dat SUMMARY FILENAME: b040511b.sum

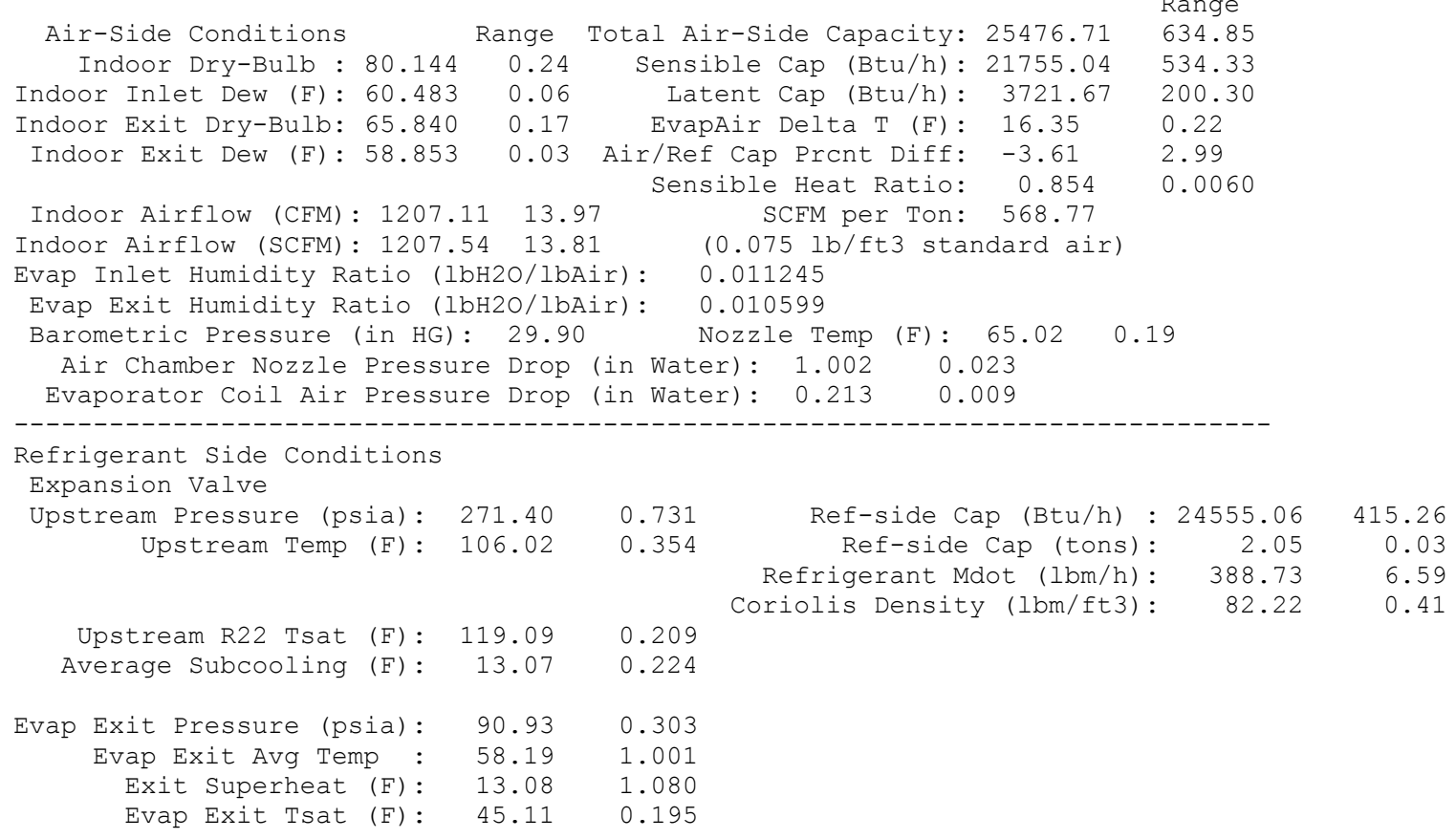


DOE/ARI MIX-MATCH EVAPORATOR TEST SUMMARY SHEET DATA FILENAME: b040513b.dat SUMMARY FILENAME: b040513b.sum

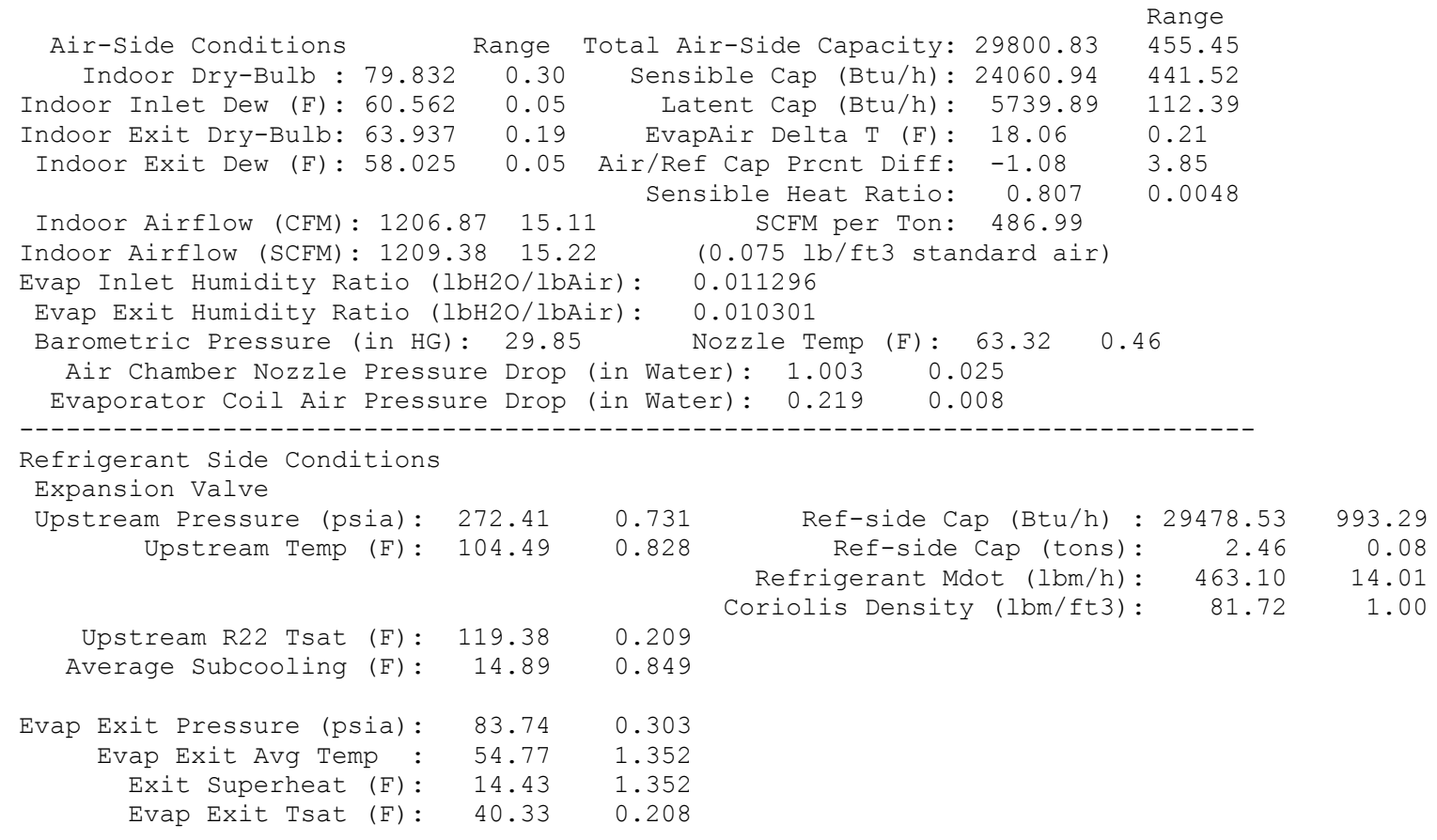


Coil 8

R22, H5321, No Fan

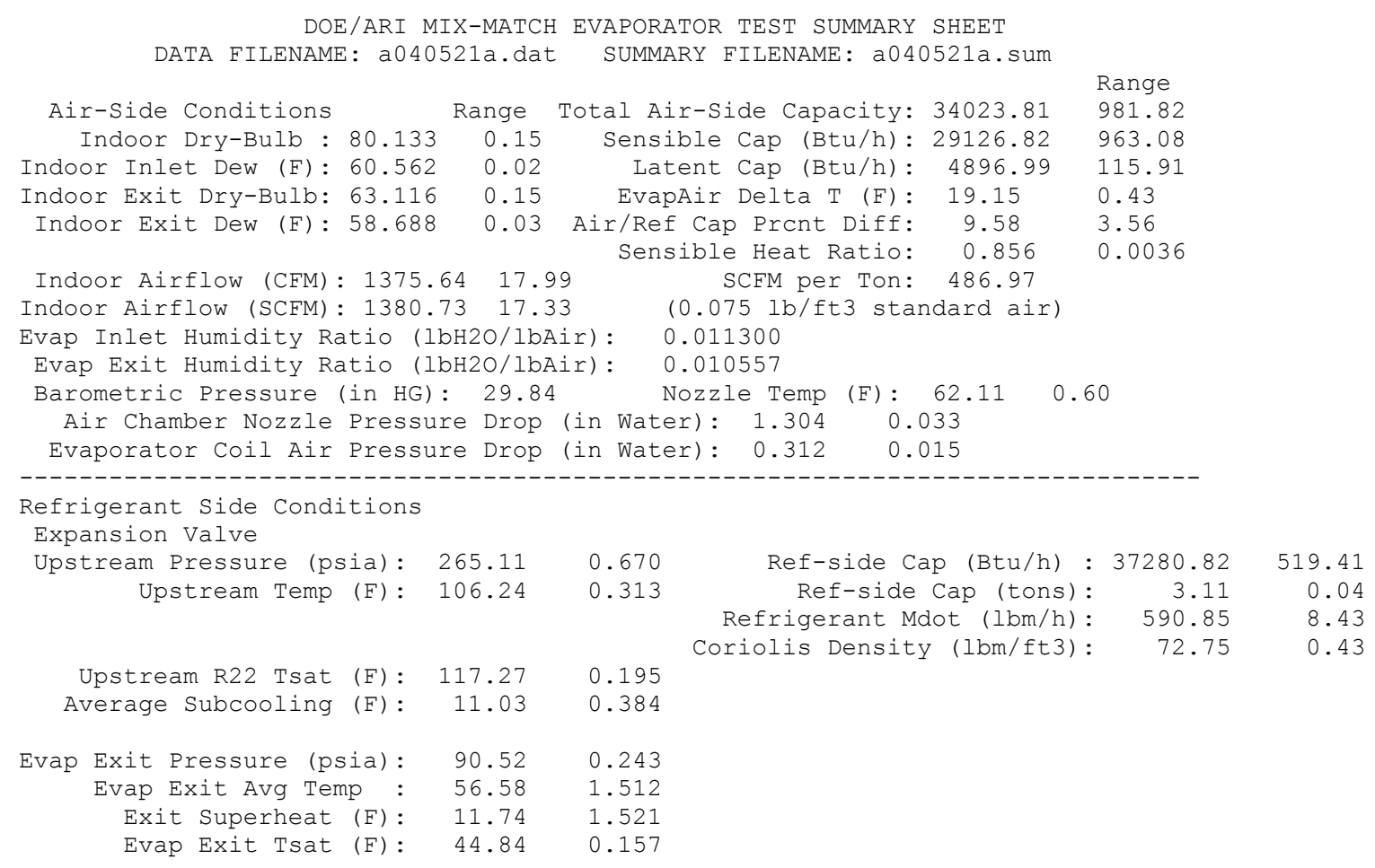


DOE/ARI MIX-MATCH EVAPORATOR TEST SUMMARY SHEET DATA FILENAME: a040525a.dat SUMMARY FILENAME: a040525a.sum

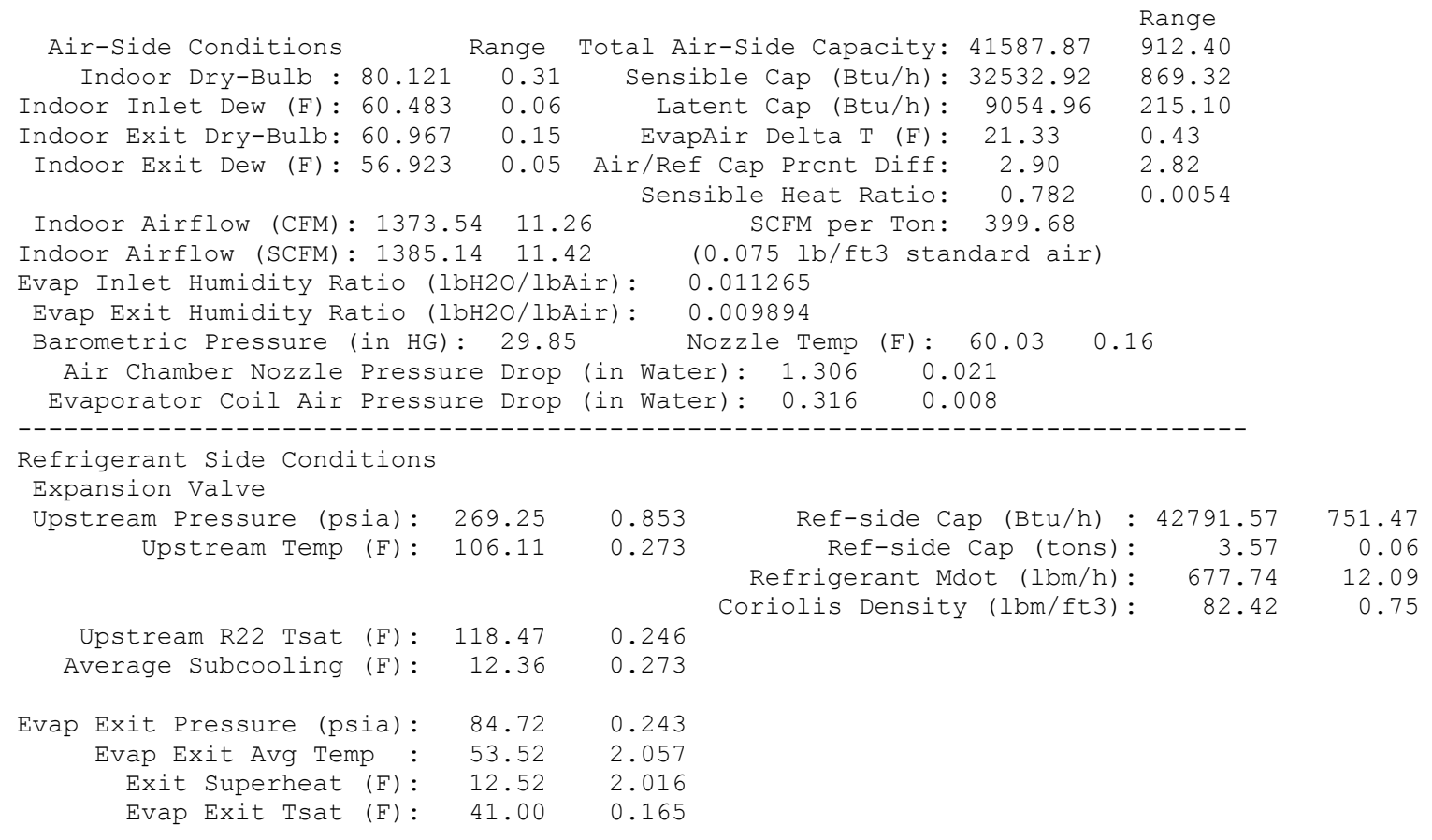


DOE/ARI MIX-MATCH EVAPORATOR TEST SUMMARY SHEET DATA FILENAME: a040526a.dat SUMMARY FILENAME: a040526a.sum

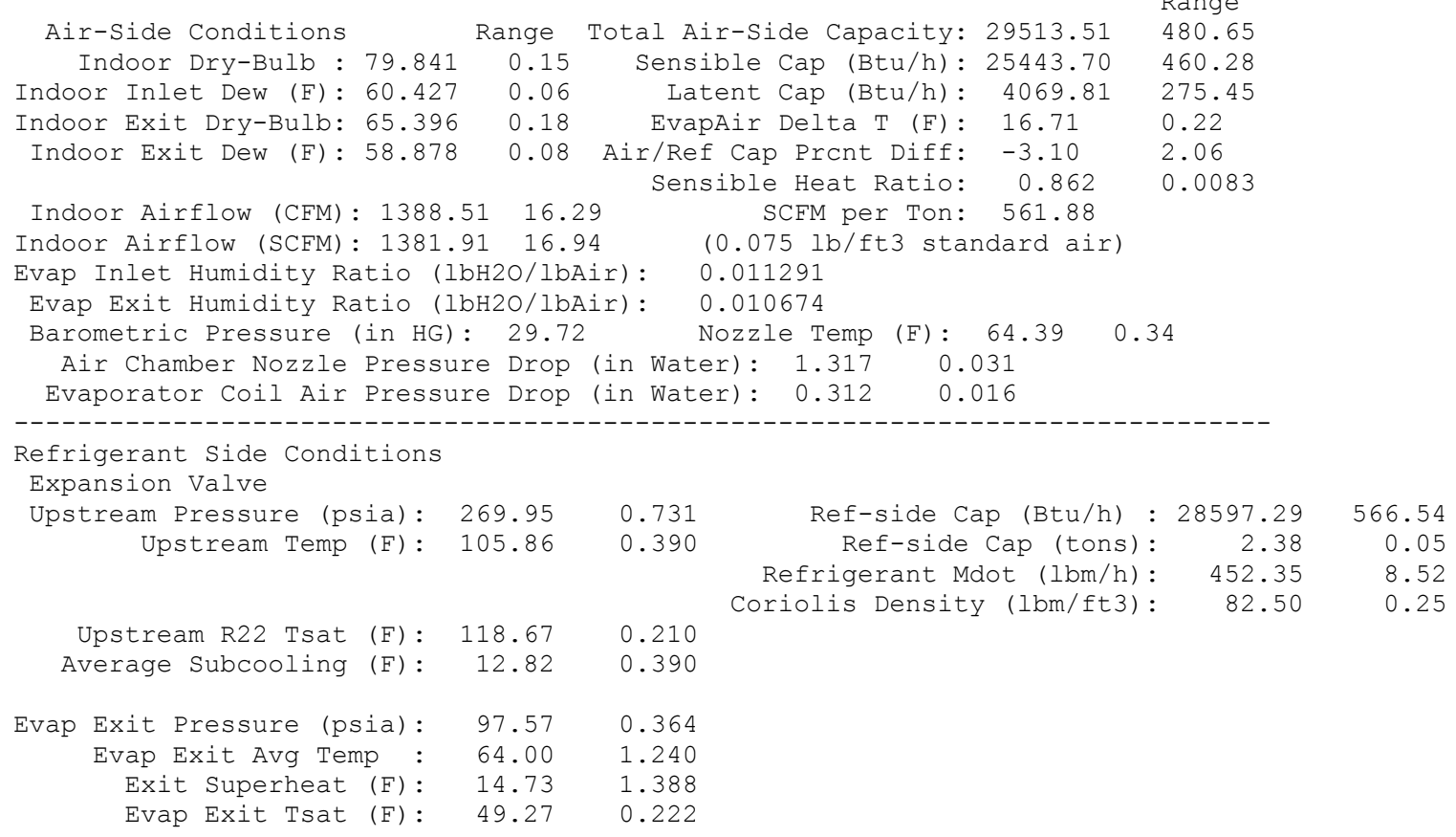


DOE/ARI MIX-MATCH EVAPORATOR TEST SUMMARY SHEET DATA FILENAME: a040527a.dat SUMMARY FILENAME: a040527a.sum

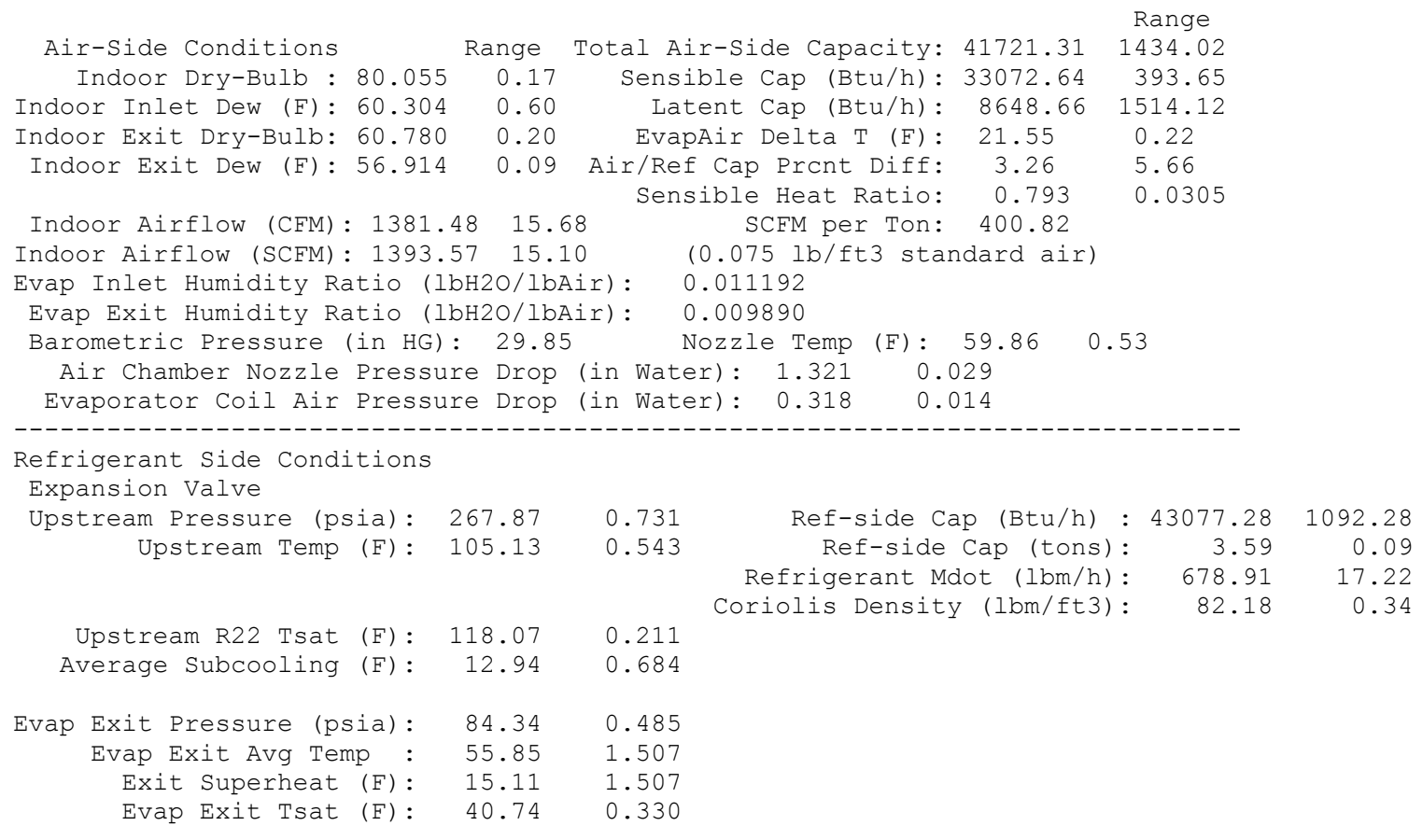


DOE/ARI MIX-MATCH EVAPORATOR TEST SUMMARY SHEET DATA FILENAME: b040527b. dat SUMMARY FILENAME: b040527b.sum

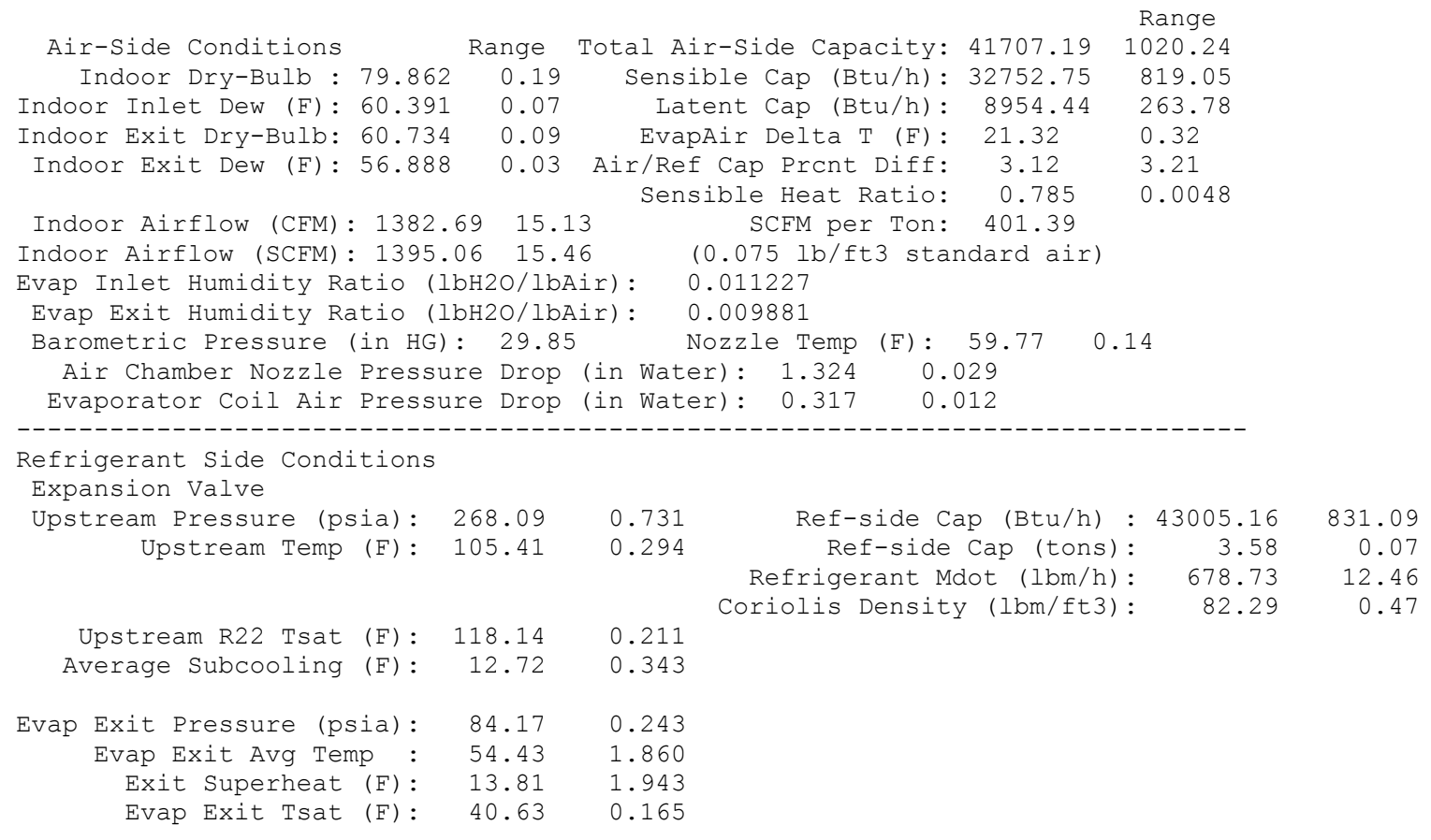


DOE/ARI MIX-MATCH EVAPORATOR TEST SUMMARY SHEET DATA FILENAME: a040603a.dat SUMMARY FILENAME: a040603a.sum

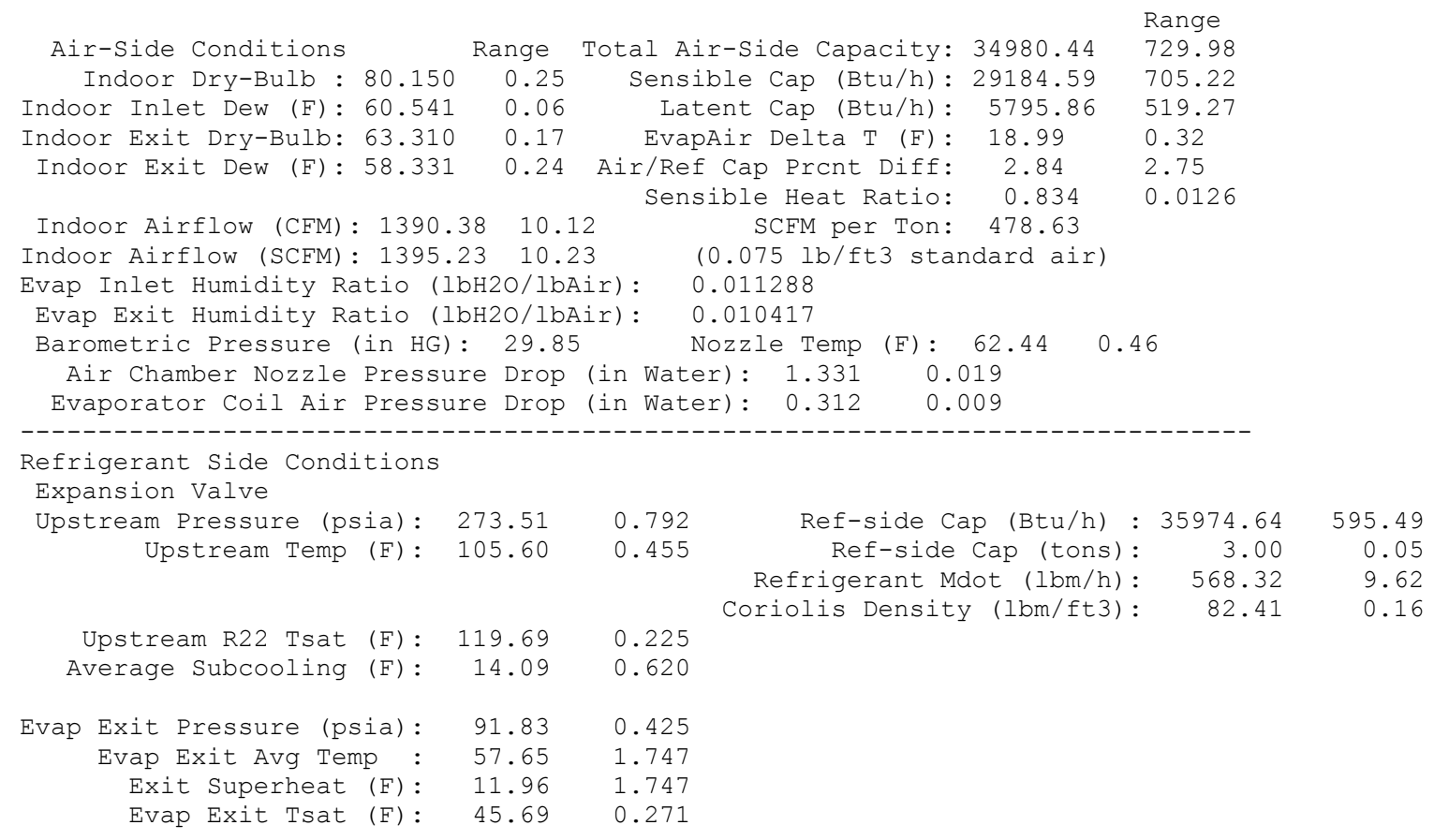


DOE/ARI MIX-MATCH EVAPORATOR TEST SUMMARY SHEET DATA FILENAME: a040607a.dat SUMMARY FILENAME: a040607a.sum

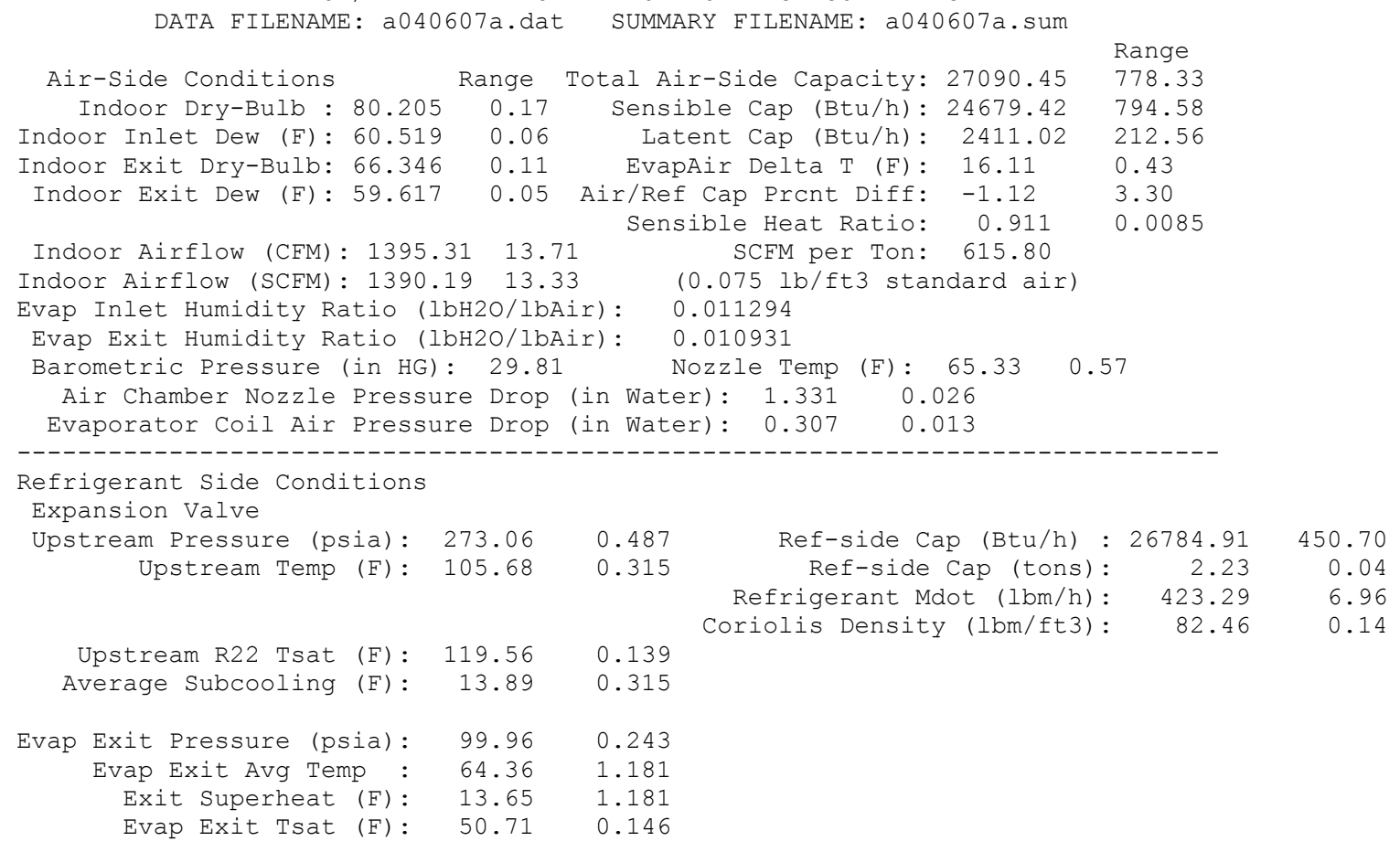




\section{Coil 9}

R410A, A01154, Fan

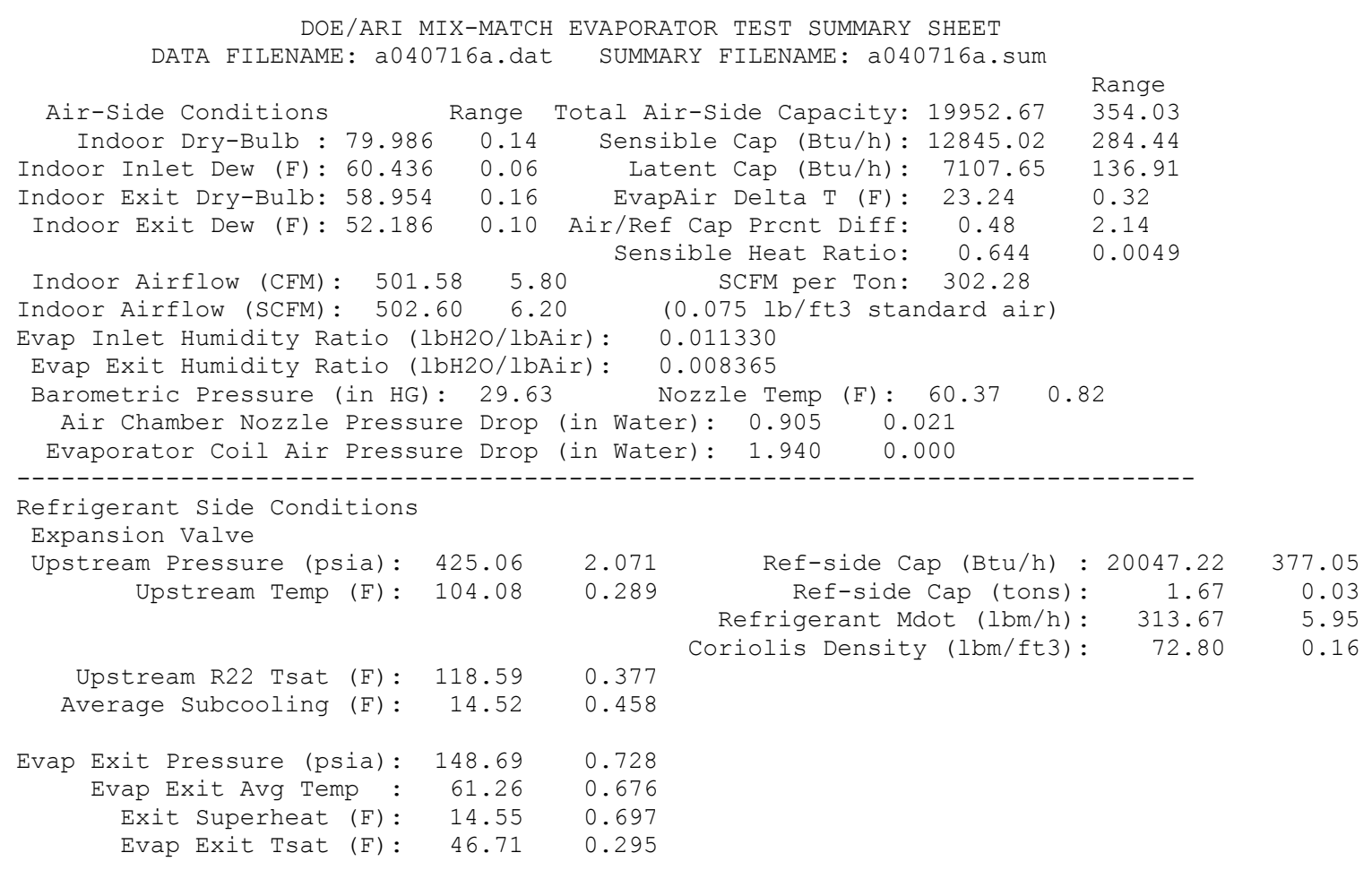


DOE/ARI MIX-MATCH EVAPORATOR TEST SUMMARY SHEET DATA FILENAME: a040728a.dat SUMMARY FILENAME: a040728a.sum

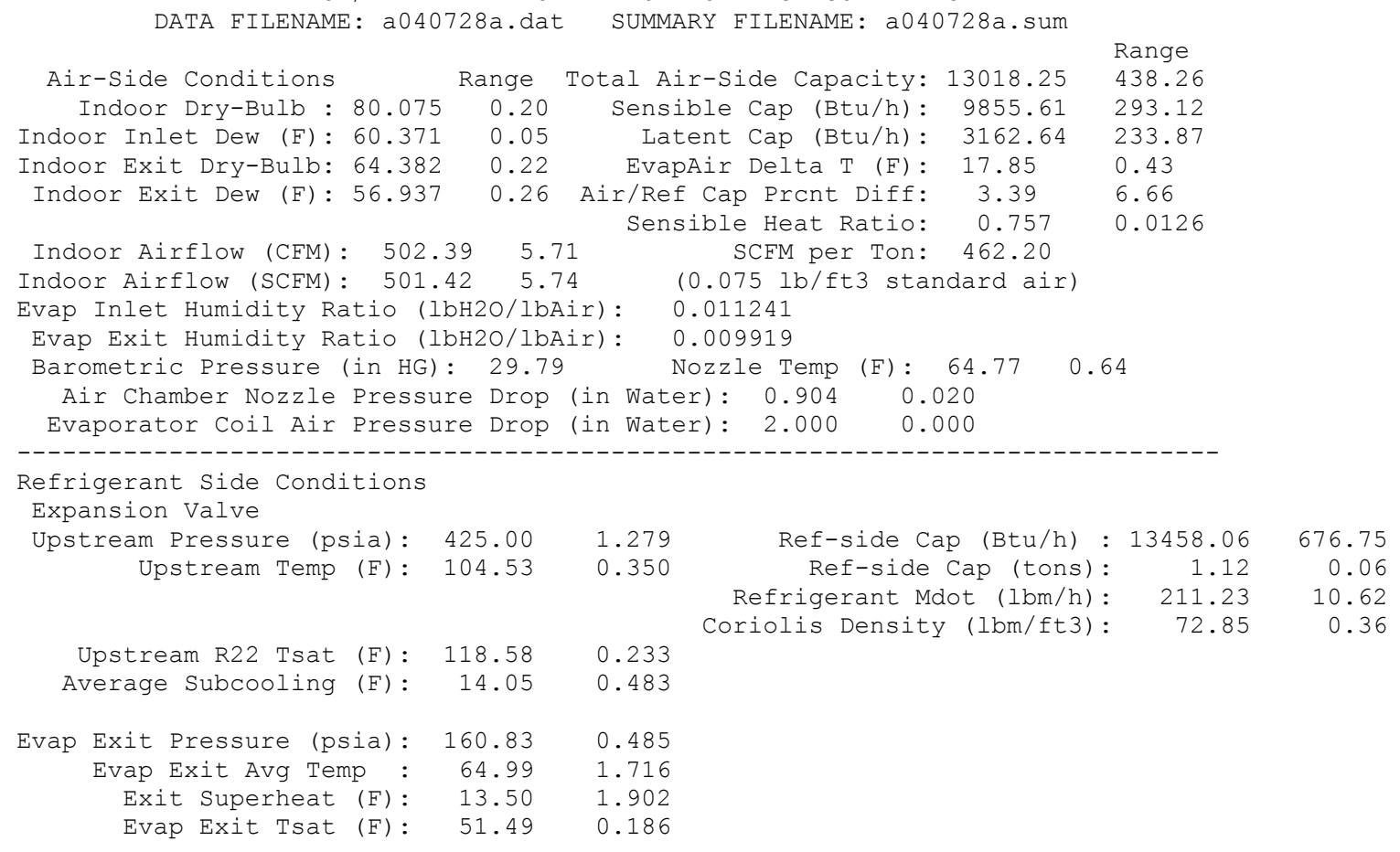


DOE/ARI MIX-MATCH EVAPORATOR TEST SUMMARY SHEET DATA FILENAME: a040729a.dat SUMMARY FILENAME: a040729a.sum

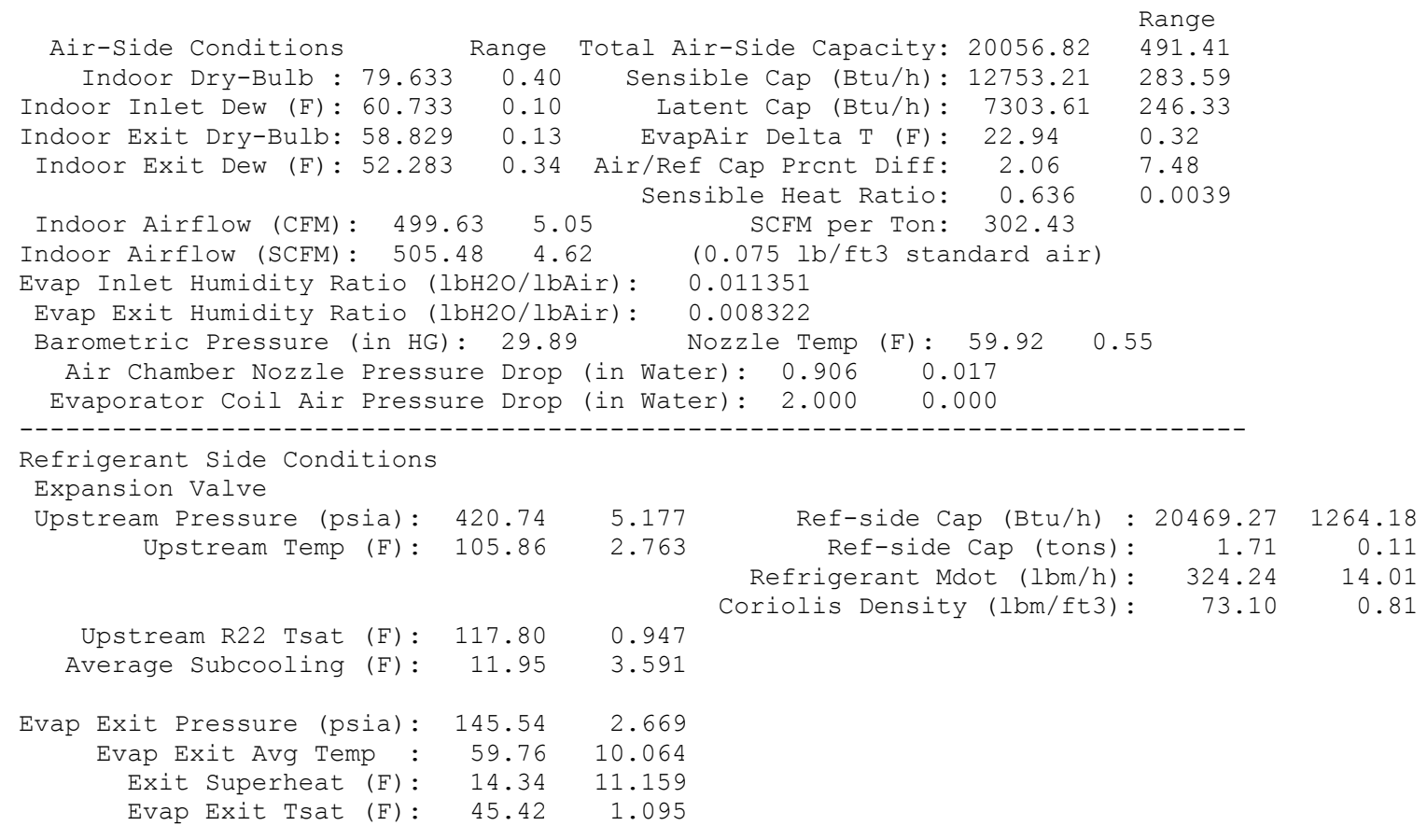


DOE/ARI MIX-MATCH EVAPORATOR TEST SUMMARY SHEET DATA FILENAME: b040729b. dat SUMMARY FILENAME: b040729b.sum

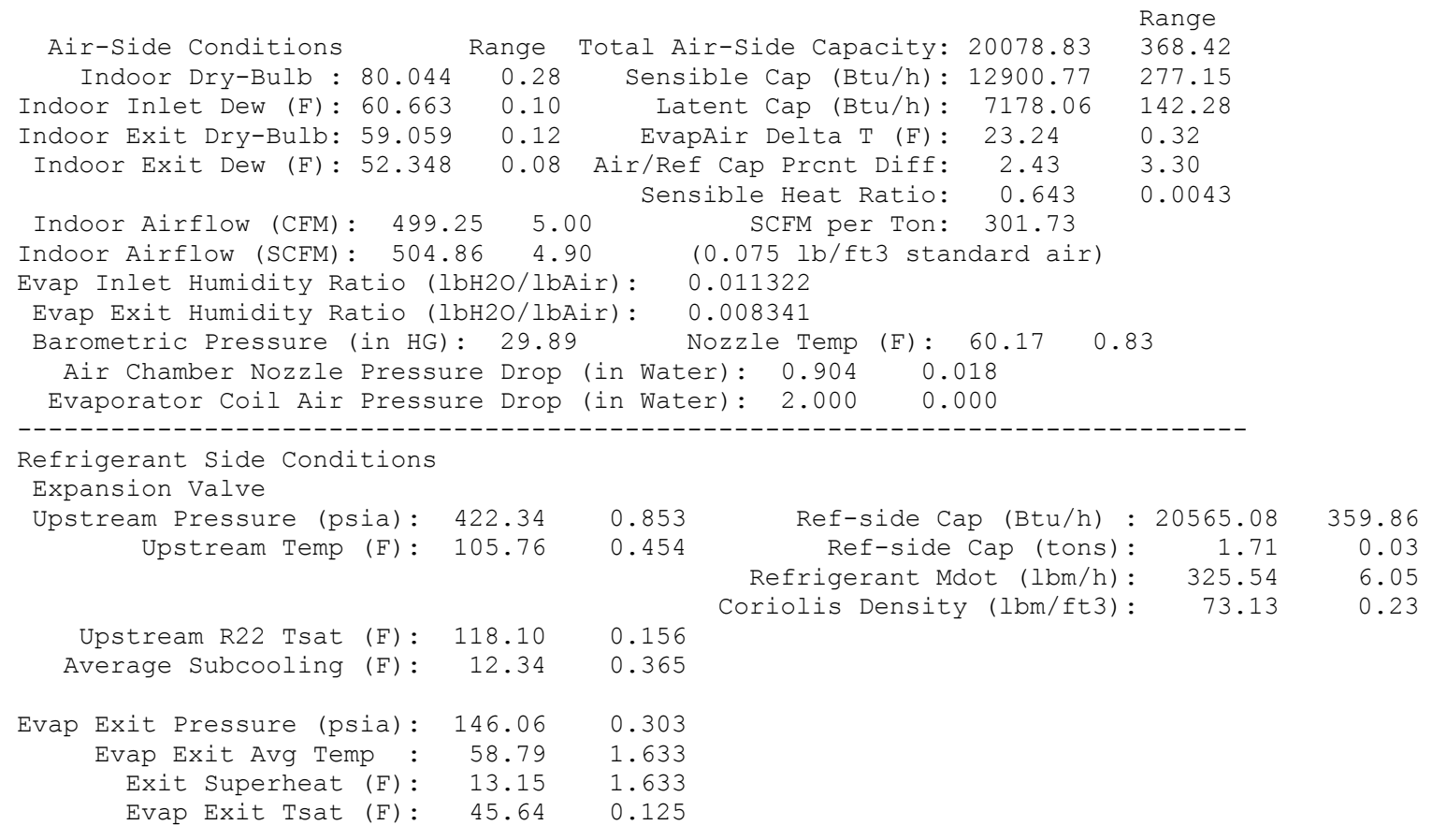


DOE/ARI MIX-MATCH EVAPORATOR TEST SUMMARY SHEET DATA FILENAME: a040730a.dat SUMMARY FILENAME: a040730a.sum

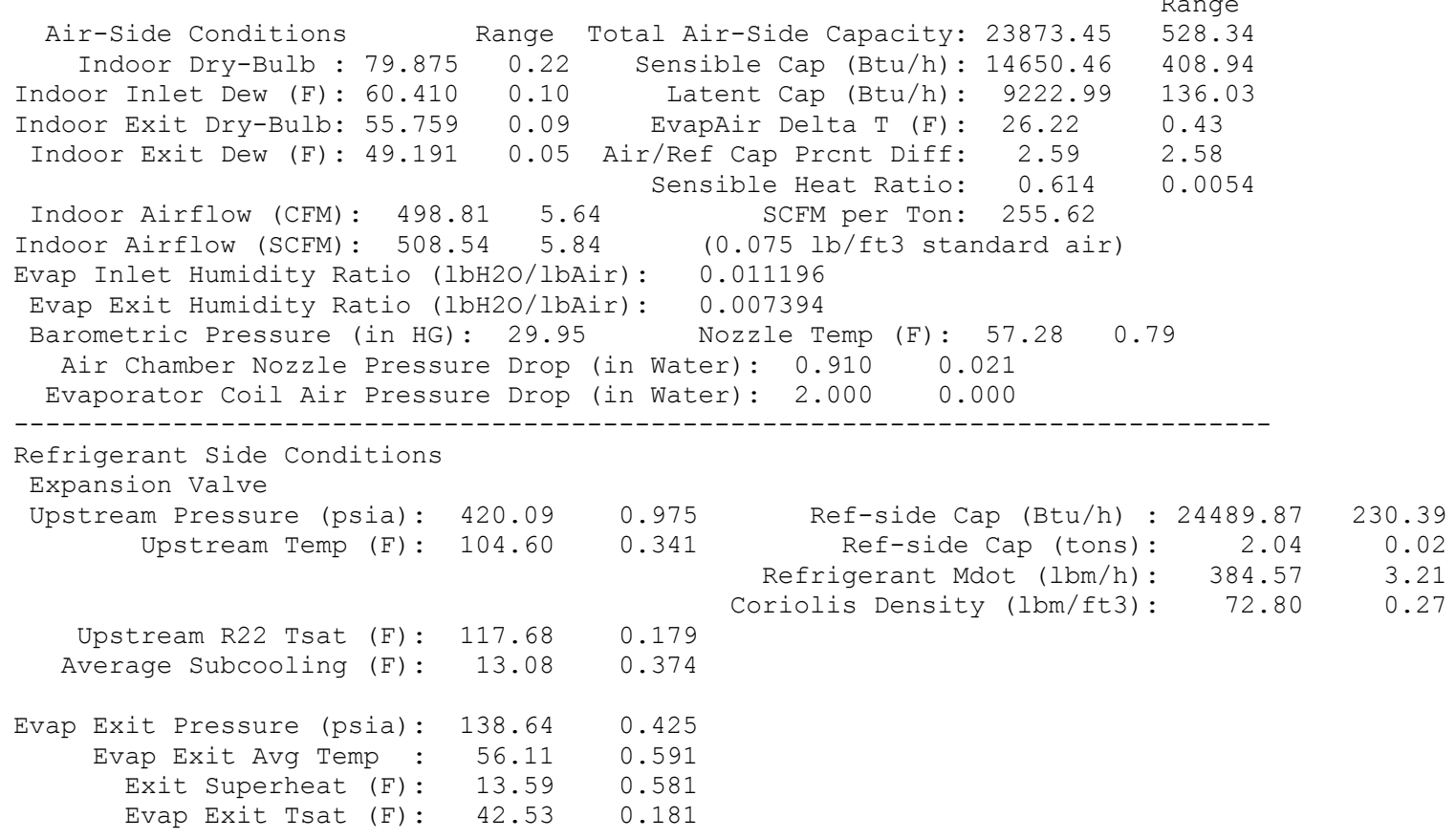


DOE/ARI MIX-MATCH EVAPORATOR TEST SUMMARY SHEET DATA FILENAME: a040803a.dat SUMMARY FILENAME: a040803a.sum

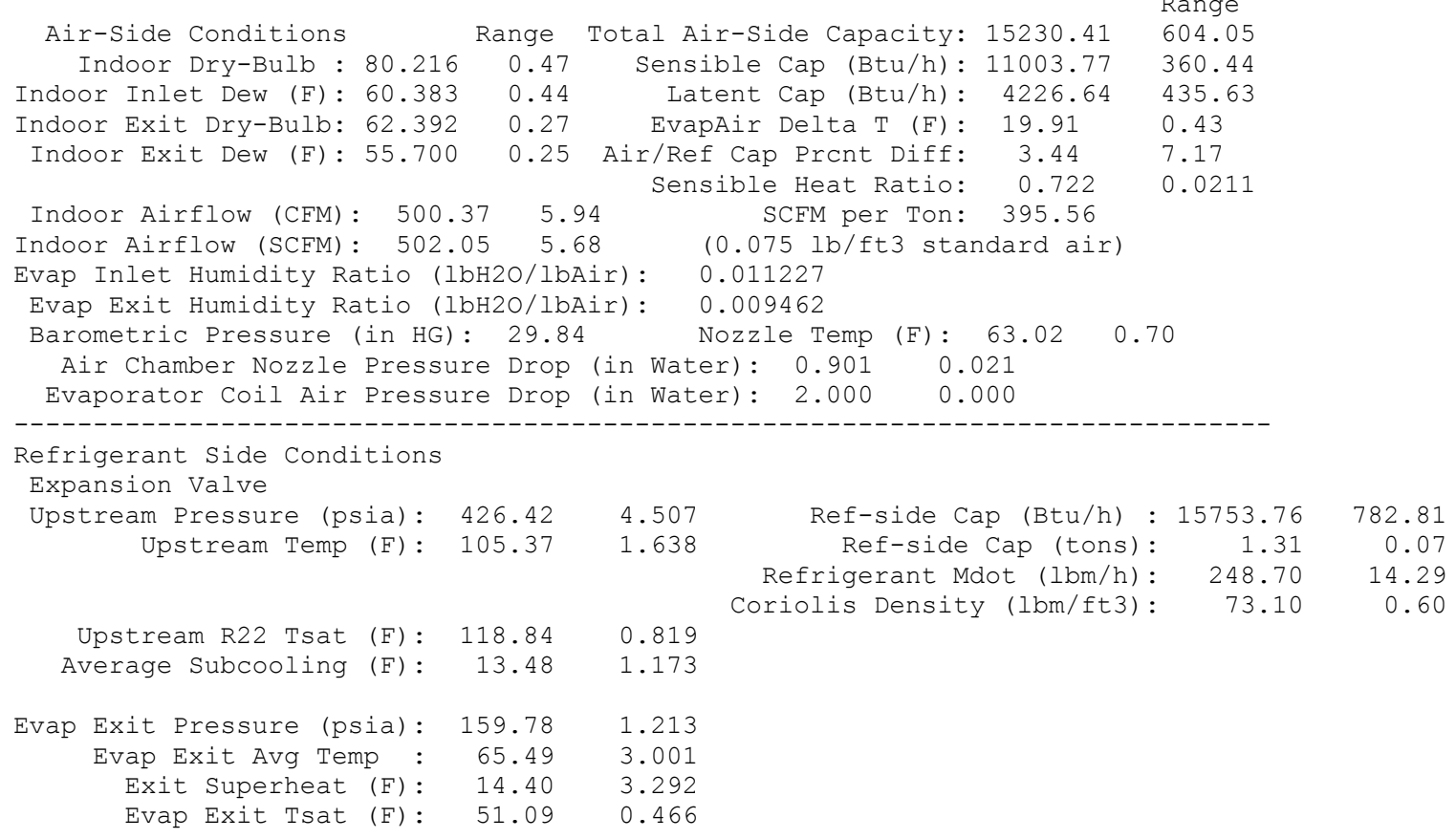


DOE/ARI MIX-MATCH EVAPORATOR TEST SUMMARY SHEET DATA FILENAME: b040803b. dat SUMMARY FILENAME: b040803b.sum

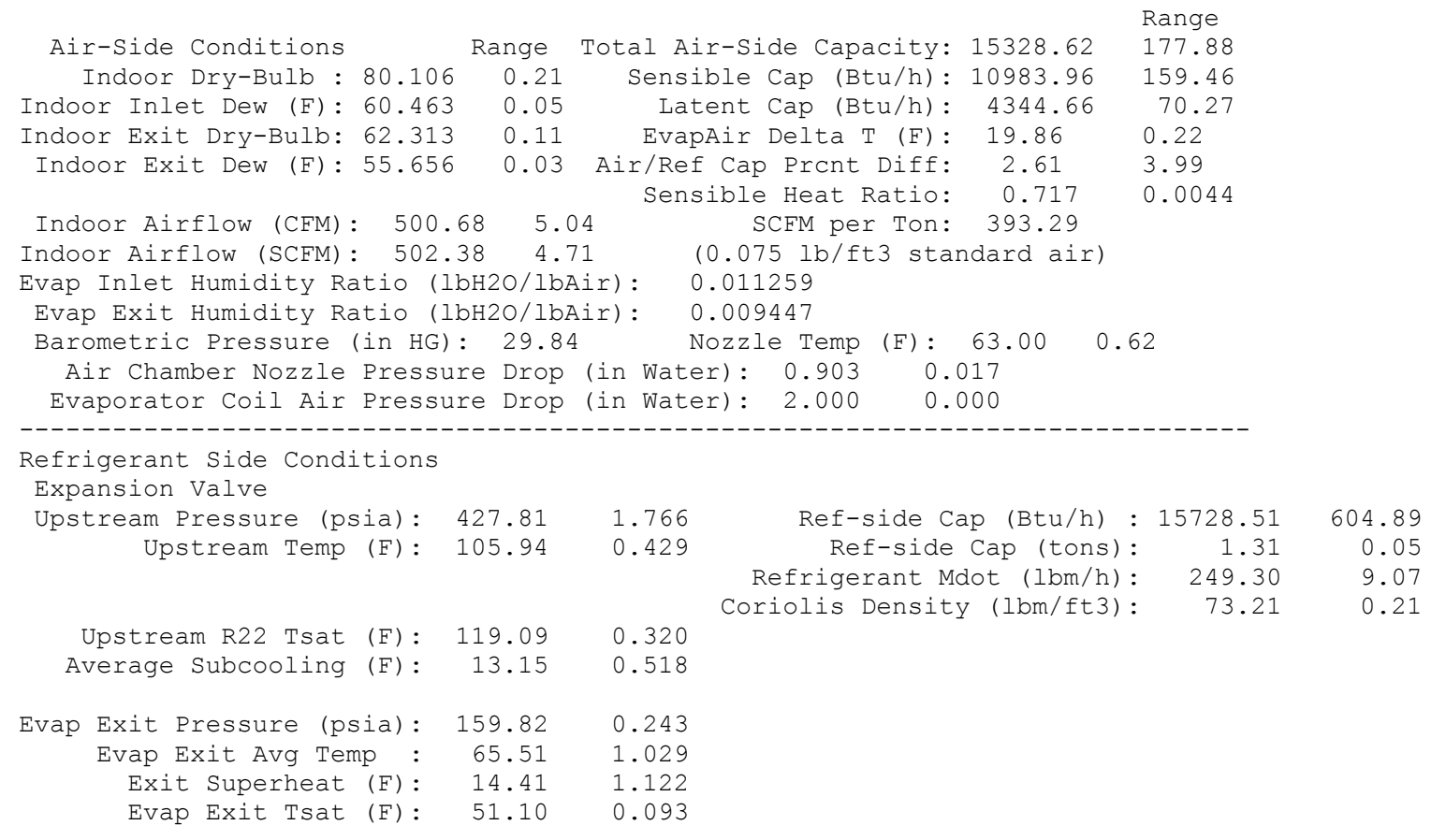


DOE/ARI MIX-MATCH EVAPORATOR TEST SUMMARY SHEET DATA FILENAME: a040817a.dat SUMMARY FILENAME: a040817a.sum

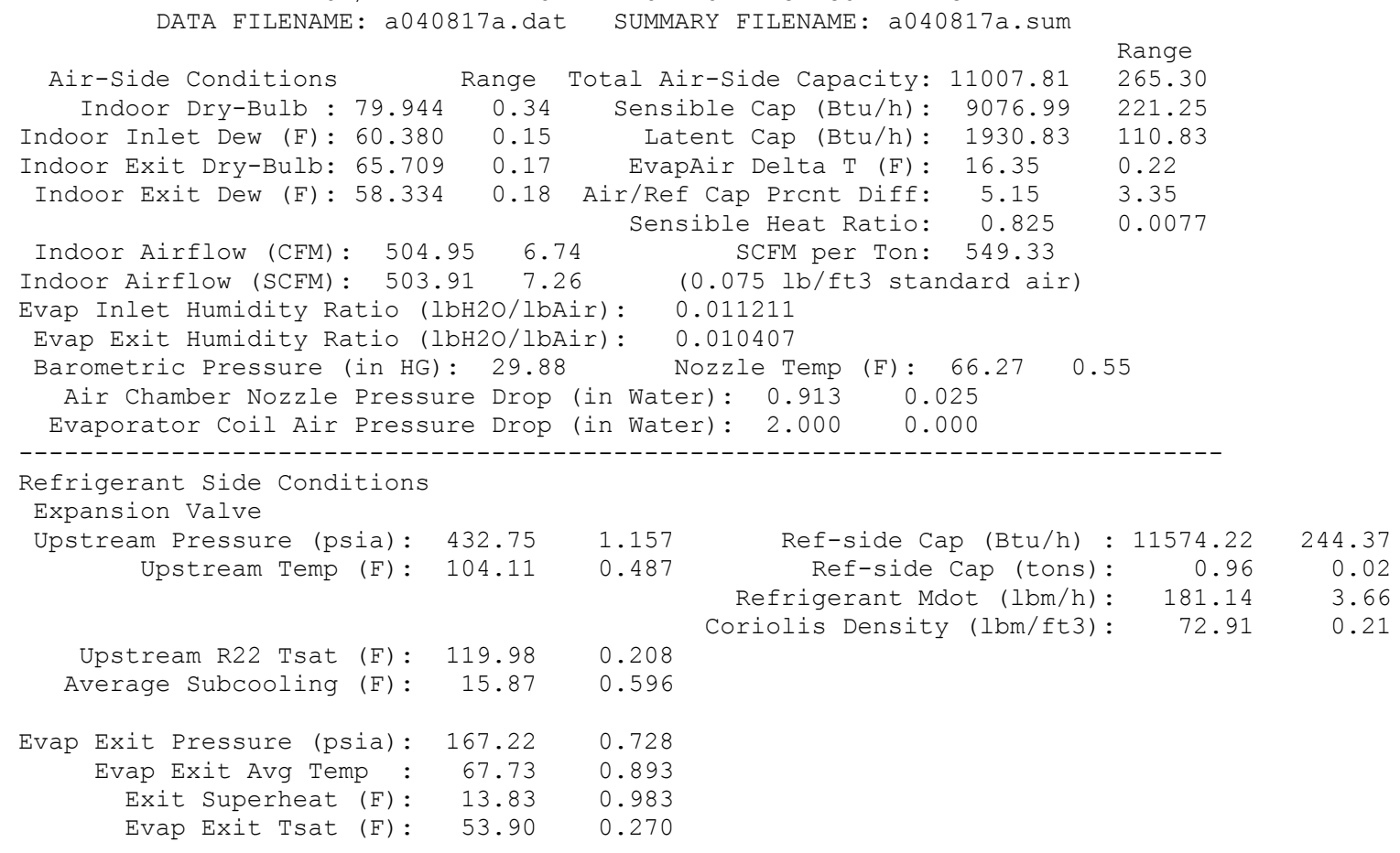

